\title{
MD 2167: Effect of a resonant excitation on the evolution of the beam emittance and halo population*
}

\author{
M. Fitterer ${ }^{\dagger}$ and G. Stancari, A. Valishev \\ Fermi National Accelerator Laboratory, PO Box 500, Batavia, Illinois 60510, USA
}

\author{
R. Bruce, S. Redaelli, B. M. Salvachua-Ferrando, D. Valuch, G. Valentino, J. F. Wagner
}

CERN, CH-1211 Geneva 23, Switzerland

(Dated: February 15, 2018)

Collimation with hollow electron beams or lenses (HEL) is currently one of the most promising concepts for active halo control in HL-LHC. In previous studies it has been shown that the halo can be efficiently removed with a hollow electron lens. Equally important as an efficient removal of the halo, is to demonstrate that the core stays unperturbed. In the case of an ideal hollow electron lens without bends, the field at the location of the beam core vanishes and the core thus remains unperturbed. In reality, the field at the beam core does not vanish entirely due to imperfections in the electron beam profile and the electron lens bends necessary to guide the electron in and out of the proton aperture. In particular, in the case of a pulsed operation of the electron lens the non-vanishing residual field induces noise on the proton beam. To identify the most sensitive pulsing patterns for the resonant mode and derive tolerances on the profile imperfections, a first MD (MD1415) was carried out on 24.08.2016 [1] and a second MD on 17.09.2017. In this note we present the results of the second MD (MD2167), which focused on confirming a part of the results of the first MD and testing in addition a resonant excitation for which no effect on the beam core is expected and the effect of random uniform noise in addition.

\footnotetext{
* Fermilab is operated by Fermi Research Alliance, LLC under Contract No. DE-AC02-07CH11359 with the United States Department of Energy. This work was partially supported by the US DOE LHC Accelerator Research Program (LARP) and by the European FP7 HiLumi LHC Design Study, Grant Agreement 284404.

$\dagger$ Email: mfittere@fnal.gov
}

This manuscript has been authored by Fermi Research Alliance, LLC under Contract No. DE-AC02-07CH11359 with the U.S. Department of Energy, Office of Science, Office of High Energy Physics. 


\section{CONTENTS}

$\begin{array}{ll}\text { I. Introduction } & 4\end{array}$

II. MD configuration and procedure $\quad 5$

A. MD configuration $\quad 5$

$\begin{array}{ll}\text { B. MD procedure } & 7\end{array}$

$\begin{array}{ll}\text { III. Results } & 7\end{array}$

A. FBCT (beam intensity) data 9

B. BWS (wire scanner) data 9

$\begin{array}{ll}\text { C. BSRT profiles at injection } & 10\end{array}$

D. BSRT emittance from the LHC Logging database 15

E. Fill 6220, $7^{\text {th }}$ turn $\quad 16$

1. Losses and loss rates from FBCT measurements 16

2. BSRT emittance from LHC logging database (Gaussian fit) 18

3. BSRT profiles $\quad 19$

4. Comparison with simulations 22

F. Fill 6220, uniform random excitation 26

1. Losses and loss rates from FBCT measurements 26

2. BSRT emittance from LHC logging database (Gaussian fit) 28

3. BSRT profiles 30

4. Comparison with simulations

G. Fill 6220, $8^{\text {th }}$ turn 36

1. Losses and loss rates from FBCT measurements 36

2. BSRT emittance from LHC logging database (Gaussian fit) 37

3. BSRT profiles 38

4. Comparison with simulations $\quad 41$

H. Influence of the transverse damper $\quad 43$

$\begin{array}{ll}\text { IV. Conclusions } & 44\end{array}$

$\begin{array}{ll}\text { V. Further analysis and outlook } & 45\end{array}$

$\begin{array}{ll}\text { VI. Acknowledgements } & 45\end{array}$

$\begin{array}{ll}\text { References } & 46\end{array}$

A. Additional figures for fill 6220, $7^{\text {th }}$ turn pusling 47

1. $7^{\text {th }}$ turn pulsing: beam losses measured with FBCT 
2. $7^{\text {th }}$ turn pulsing: BSRT emittance as logged in the LHC Logging database 48

3. $7^{\text {th }}$ turn pulsing: BSRT profile analysis

a. $7^{\text {th }}$ turn pulsing: BSRT profiles - statistical parameters 53

b. $7^{\text {th }}$ turn pulsing: BSRT profiles - q-Gaussian fit parameters in the horizontal plane 57

c. $7^{\text {th }}$ turn pulsing: BSRT profiles - q-Gaussian fit parameters in the vertical plane 63

d. $7^{\text {th }}$ turn pulsing: BSRT profiles of representative bunches 69

4. $7^{\text {th }}$ turn pulsing: FMA Analysis for 2017 optics and tune 75

B. Additional figures for fill 6220, random excitation 76

1. Random excitation: Beam losses measured with FBCT 76

2. Random excitation: BSRT emittance as logged in the LHC Logging database 77

3. Random excitation: BSRT profile analysis $\quad 82$

a. Random excitation: BSRT profiles - statistical parameters $\quad 82$

b. Random excitation: BSRT profiles - q-Gaussian fit parameters in the horizontal plane 86

c. Random excitation: BSRT profiles - q-Gaussian fit parameters in the vertical plane 95

d. random excitation: BSRT profiles of representative bunches 104

4. random excitation: FMA Analysis for 2017 optics and tune 110

C. Additional figures for fill 6220, $8^{\text {th }}$ turn pusling 111

1. $8^{\text {th }}$ turn pulsing: beam losses measured with FBCT 111

2. $8^{\text {th }}$ turn pulsing: BSRT emittance as logged in the LHC Logging database 112

3. $8^{\text {th }}$ turn pulsing: BSRT profile analysis $\quad 117$

a. $8^{\text {th }}$ turn pulsing: BSRT profiles - statistical parameters 117

b. $8^{\text {th }}$ turn pulsing: BSRT profiles - q-Gaussian fit parameters in the horizontal plane $\quad 121$

c. $8^{\text {th }}$ turn pulsing: BSRT profiles - q-Gaussian fit parameters in the vertical plane $\quad 130$

d. $8^{\text {th }}$ turn pulsing: BSRT profiles of representative bunches 139

4. $8^{\text {th }}$ turn pulsing: FMA Analysis for 2017 optics and tune 145 


\section{INTRODUCTION}

For high energy and high intensity hadron colliders like the HL-LHC, halo control becomes necessary, for a safe machine operation and control of the targeted stored beam energy in the range of several hundred MJ [2]. Past experiments at the Fermilab Tevatron proton-antiproton collider [3] demonstrated a successful halo control with hollow electron beams or hollow electron lenses (HELs) in DC mode. Simulations of the HEL performance for LHC and HL-LHC [4-6] show sufficiently high halo removal rate with the HEL operated in DC mode if beams are colliding, but only very low halo removal rates if beams are separated. In order to clean the tails efficiently with the HEL and in a short time-span also in case of separated beams, the halo removal rate can be increased by pulsing the HEL $[4,6,7]$, where two different pulsing patterns are considered:

- random: the e-beam current is modulated randomly: at every turn the kick is varied between 0 and its maximum value following a uniform distribution. Instead of modulating between 0 and the maximum value, also only a fraction of the current can be modulated.

- resonant: the e-lens is switched on only every $n^{\text {th }}$ turn with $n=2,3,4, \ldots$ and the maximum kick is applied.

One of the main reservations about pulsing the e-lens is the possibility of emittance growth due to noise induced on the beam core by the HEL. For an ideal radially symmetric hollow electron lens with an Sshaped geometry, the beam core would experience a zero net kick and thus no noise would be induced on the core (see Fig. 1 for the HEL design and for a sketch of the HEL field). In the presence of imperfections in the HEL bends and in the e-beam profile, the kick at the center of the beam is non-zero. First estimates of the residual kick yield $0.5 \mathrm{nrad}$ from the HEL bends assuming $10 \%$ difference between entrance and exit bend $^{1}$ and 15 nrad due to profile imperfections based on profile measurements of the current HEL e-gun
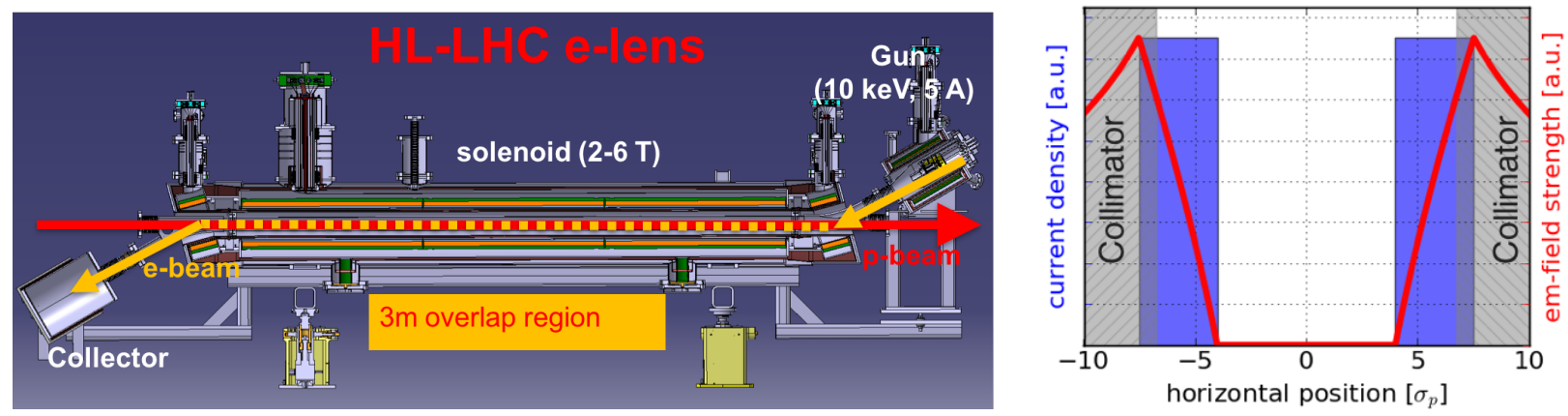

Figure 1. (left) Layout of the hollow electron lens for HL-LHC (left) and sketch of the HEL current density, electromagnetic field strength and collimator aperture for HL-LHC (right).

\footnotetext{
${ }^{1}$ Without any imperfections the kick from the e-lens bends at the entrance and exit would compensate each other in case of a S-shaped e-lens and add up in case of an U-shaped e-lens. For the HL-LHC therefore a S-shaped design is chosen. Due to for example field imperfections in the solenoids, space-charge effects or alignment errors, the kick at the entrance and exit can differ. As a first guess a difference of $10 \%$ between entrance and exit kick is assumed.
} 
prototype [8]. The estimates for the profile imperfections are likely to be pessimistic as also alignment errors of the solenoids of the test stand and orbit deviations can contribute to the field at the beam center. There is currently an effort ongoing to improve the profile measurements and reduce these effects.

In case of DC operation of the HEL the kicks are static and could thus be corrected, if even necessary. However, for a pulsed operation, the tolerable kick amplitudes are much smaller as then noise is introduced also on the beam core. In case of random excitation, white noise is induced driving all orders of resonances. In case of a resonant pulsing, only certain resonances are driven, explicitly for pulsing every $n^{\text {th }}$ turn only the $n^{\text {th }}$ turn order resonances are driven, which can be seen for example from the Fourier series. The $n^{\text {th }}$ turn excitation can be represented by

$$
f(t)=\sum_{p=-\infty}^{+\infty} \delta(t-p \cdot(n T))
$$

and its Fourier series is then given by:

$$
f(t)=\frac{1}{n T} \sum_{k=-\infty}^{+\infty} e^{2 \pi i f_{k} t} \text { with } f_{k}=\frac{k}{n} f_{\text {rev }}
$$

Expected effects of the resonant excitation as well as from a random uniform modulation are losses and emittance growth.

The effect of a resonant excitation, explicitly pulsing every 7 th and 10th turn, has been explored in a previous MD on 23.08.2016/24.08.2016, fill numbers 5242 and 5243 [1]. In this MD, the aim was to confirm part of the results of the previous MD [1] and test in addition the random modulation in comparison to the resonant excitation and one resonant excitation pattern, for which no effect on the beam core is expected. In summary the following excitation patterns were tested:

- 7 th turn pulsing: to confirm the results of the previous MD [1]

- $8^{\text {th }}$ turn pulsing: to test one new excitation pattern for which no effect on the beam is expected

- uniform random excitation: to compare the effect of a random excitation with a resonant excitation

In order to compare the obtained results with the previous MD [1], the same machine configuration has been chose as far as possible. For a list of changes, see II A.

The MD configuration and procedure are described in further detail in Sec. II and the MD results are summarized in Sec. III.

\section{MD CONFIGURATION AND PROCEDURE}

\section{A. MD configuration}

In order to keep the machine changes minimal, the standard injection optics is used, which was changed to ATS optics and is thus different compared to the previous MD. We had to also change the working point as 
for the fractional tune of $\left(Q_{x}, Q_{y}\right)=(.27, .31)$ as used in the previous $\mathrm{MD}$, high losses were observed, which would have compromised the experiment. Instead the standard injection tune of $\left(Q_{x}, Q_{y}\right)=(62.27,60.295)$. Note that also the integer part of the tune for the two MDs is different. As in particular the resonant excitation depends on the excited resonances, a change in tune will also change the effect of the excitation. The simulations done for the previous MD [8] therefore also have to be repeated. Also we used more bunches in order to test excitation in $\mathrm{H}, \mathrm{V}$ and $\mathrm{H}+\mathrm{V}$ at the same time and also increase statistics by using 6 bunches with the same pattern and amplitude instead of previously 4 . Otherwise the same settings and parameters as in the previous are used. Explicitly, for both experiments also Beam 1 was used. All parameters for both MDs are summarized in Table I. The noise induced by a pulsed e-lens can be approximated to first order

Table I. Beam parameters and machine configuration as used in the two resonant excitation MDs.

\begin{tabular}{|l|c|c|}
\hline Parameter & MD 2167 (17./18.09.2017) & MD 1415 (23./24.08.2016) \\
\hline Beam & \multicolumn{2}{|c|}{ Beam 1 } \\
\hline beam energy & \multicolumn{2}{|c|}{ injection energy, 450 GeV } \\
\hline single bunch intensity & \multicolumn{2}{|c|}{$0.7 \times 10^{11}$} \\
\hline normalized emittance & \multicolumn{2}{|c|}{$2.5-3.5 \mu \mathrm{m}$} \\
\hline number of bunches & $\begin{array}{c}3 \times 72=216 \text { bunches } \\
(+1 \text { pilot }+12 \text { nominal })\end{array}$ & $12 \times 4=48$ single bunches \\
\hline injection optics, $\beta^{*}=11 \mathrm{~m}$ & standard optics 2017 & standard optics 2016 \\
\hline working point $\left(Q_{x}, Q_{y}\right)$ & $(62.27,60.295)$ & $(64.28,59.31)$ \\
\hline chromaticity $\left(Q_{x}^{\prime}, Q_{y}^{\prime}\right)$ & \multicolumn{2}{|c|}{$(+15,+15)$} \\
\hline Landau damping octupoles & $I_{\mathrm{MO}}= \pm 19.6$ A, explicitly +19.6 A for MOF circuit and \\
& -19.6 A for MOD circuit (standard 2016 settings $)$ \\
\hline
\end{tabular}

by a dipole kick with the corresponding noise pattern/frequency spectrum [8]. In case of the LHC almost arbitrary noise spectra seen by the whole beam can be generated using the transverse damper (ADT) and the amplitude of the noise seen by individual bunches can be controlled by a windowing function placed on top of the generated excitation. In the previous MD the kick amplitude could only be determined roughly within $50 \%$ of the excitation amplitude. Thanks to a better calibration this error could be reduced to $10 \%$. In addition the horizontal and vertical kickers can be controlled individually, which allows for testing the same excitation in $\mathrm{H}, \mathrm{V}$ and $\mathrm{H}+\mathrm{V}$ during the same fill by exciting only certain bunches. With an especially for this MD developed filling scheme [9], we were able to test in total:

- 3 different excitations: $7^{\text {th }}$ turn, $8^{\text {th }}$ turn, uniform random

- 6 different excitation amplitudes per pattern

- each pattern with excitation only in $\mathrm{H}$, only in $\mathrm{V}$ and in $\mathrm{H}+\mathrm{V}$

- each pattern and plane/plane combination with and without the transverse damper active 
- 6 bunches for each pattern - plane - damper active/not active - amplitude constellation

The final filling scheme with the excitation pattern overlain is shown in Fig. 2. As a first estimate of the kick
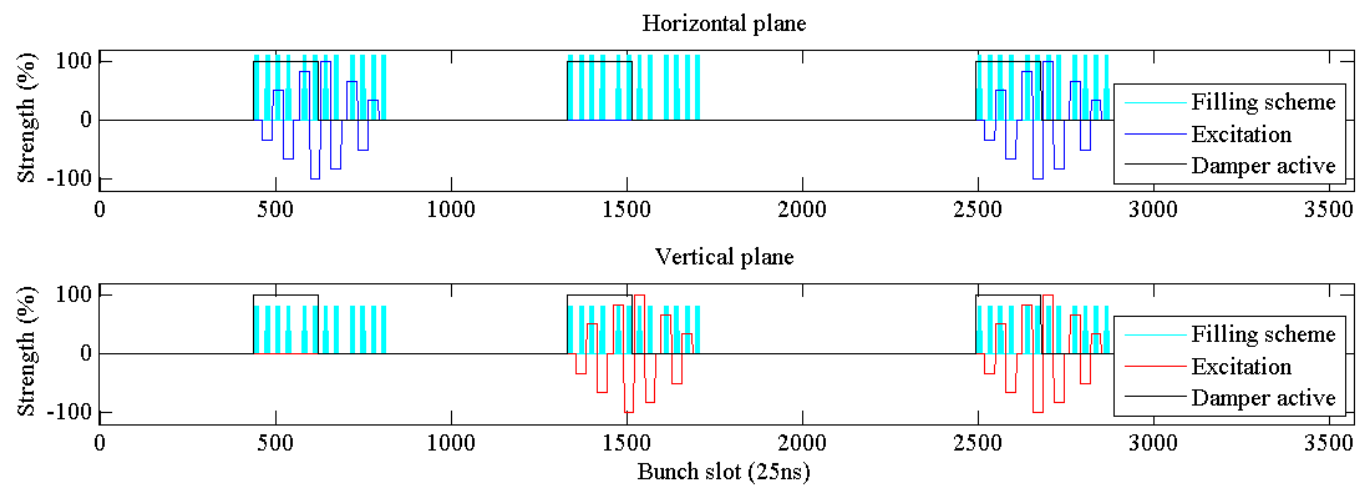

Figure 2. Filling scheme used for this MD without the 1 pilot bunch and the 12 nominal bunches, which are only necessary for the relevant injection checks. Each light blue line consists of 6 individual bunches spaced by $50 \mathrm{~ns}$.

amplitude, we used the range of amplitudes obtained in the previous MD. The smallest amplitudes for $7^{\text {th }}$ turn pulsing were 6 nrad and the largest amplitude and also maximum amplitude achievable with the ADT in order not to have saturation issues is 96 nrad. For the random excitation we started with amplitudes as small as $0.5 \mathrm{nrad}$ as the effect of this excitation is expected to be much stronger. The error on the excitation amplitude during the previous MD 1415 is estimated to be around 50\% and could be reduced in this MD to $10-15 \%$.

\section{B. MD procedure}

A first test of the ADT excitation with only pilot bunches was conducted before exciting the full beam. The measurements themselves then consisted of three injections during fill 6220 . For each pattern $\left(7^{\text {th }}\right.$ turn, uniform random, $8^{\text {th }}$ turn), the beam was dumped and a new beam reinjected in order to start with an unchanged beam distribution. This we learned as a lesson from the last MD, where we had to basically discard the results for the 8 th and 3rd turn pulsing as the beam distribution was already too heavily changed. The detailed time line is summarized in Table II.

\section{RESULTS}

During the MD the bunch-by-bunch beam losses were recorded with the Fast Beam Current Transformer (FBCT) and the diamond detectors. The emittance was measured using the Beam Synchrotron Radiation Telescope (BSRT) and the wire scanners. In addition to the emittance measurements, also the horizontal and vertical BSRT profiles were recorded in order to obtain more information about eventual changes in the beam distribution and a summary of the analysis of these profiles is given in the following preceded by 
Table II. Time line and list of excitation patterns and amplitudes during the MD. Times are given in Europe/Zurich time.

\begin{tabular}{|c|c|c|c|c|}
\hline fill number & injection & time & pulsing pattern & amplitude [nrad] \\
\hline \multirow[t]{9}{*}{6219} & \multirow{9}{*}{ inj 1} & $19: 56-20: 26$ & \multirow{4}{*}{\multicolumn{2}{|c|}{$\begin{array}{l}\text { setup machine with probe: } \\
\left(Q_{x}, Q_{y}\right)=(64.28,59.31) \\
\left(Q_{x}^{\prime}, Q_{y}^{\prime}\right)=(+15,+15) \\
I_{\mathrm{MO}}= \pm 19.6 \mathrm{~A}\end{array}$}} \\
\hline & & & & \\
\hline & & & & \\
\hline & & & & \\
\hline & & $20: 26$ & \multicolumn{2}{|c|}{ testing ADT excitation with probes } \\
\hline & & $20: 37$ & \multirow{4}{*}{\multicolumn{2}{|c|}{$\begin{array}{l}\text { set tunes back to } \\
\left(Q_{x}, Q_{y}\right)=(62.27,60.295) \text { as for } \\
\left(Q_{x}, Q_{y}\right)=(64.28,59.31) \text { losses are } \\
\text { very high. }\end{array}$}} \\
\hline & & & & \\
\hline & & & & \\
\hline & & & & \\
\hline \multirow[t]{12}{*}{6220} & \multirow{4}{*}{ inj 1} & $21: 21-21: 40$ & \multicolumn{2}{|c|}{ no excitation, let distribution adjust } \\
\hline & & $21: 40-21: 55$ & \multirow{2}{*}{$7^{\text {th }}$ turn } & 6 \\
\hline & & $21: 56-22: 07$ & & 12 \\
\hline & & $22: 07-22: 23$ & random (test) & $1-20$ \\
\hline & \multirow{4}{*}{ inj 2} & $22: 46-22: 57$ & no excitation, le & tistribution adjust \\
\hline & & $22: 57-23: 10$ & \multirow{3}{*}{ random } & 1 \\
\hline & & $23: 10-23: 25$ & & 6 \\
\hline & & $23: 25-23: 30$ & & 12 \\
\hline & \multirow{4}{*}{ inj 3} & $00: 13-00: 25$ & no excitation, le & t distribution adjust \\
\hline & & $00: 25-00: 30$ & \multirow{3}{*}{$8^{\text {th }}$ turn } & 12 \\
\hline & & $00: 30-00: 41$ & & 36 \\
\hline & & $00: 41-00: 51$ & & 96 (max achievable amp) \\
\hline
\end{tabular}

a short introduction to the BSRT profile analysis in Sec. III C. Another method of tail measurements is to operate the wire scanners in saturated mode. As this method was not successful during the last MD, we did not use it in this MD and explicitly used the wire scanners only for emittance measurement. Unfortunately there was a bug in the logging which is currently being investigated, so that all relevant wire scans taken during the MD were not saved in the LHC logging database (see Sec. III B).

In this section, the results are presented in chronological order for the different pulsing patterns (see Table II for the time line). Detailed descriptions of the definitions and analysis are only given in the first analysis (Sec. III E). For each pulsing pattern the following analysis is performed:

1. analysis of loss rates using the bunch-by-bunch intensity measured with the FBCTs

2. analysis of loss rates using the diamond bunch-by-bunch loss monitors. This analysis is still to be done.

3. analysis of the normalized bunch-by-bunch emittance with the beam wire scanners (BWS). This analysis could not be performed as all data was lost due to a logging error (see Sec. III B). 
4. analysis of the normalized bunch-by-bunch emittance measured with the BSRT and as logged in the LHC logging database. In this case, the emittance is obtained via an automatic Gaussian fit to the profiles.

5. in detail analysis of the BSRT profiles by calculating different statistical parameters and comparing the evolution of the profiles with time. A summary of the analysis is given in Sec. IIIC and more details of the BSRT profile analysis can be found in [10].

As a final chapter, the effect of the transverse damper in the presence of $n^{\text {th }}$ turn pulsing and a resonant excitation is described (Sec. III H).

\section{A. FBCT (beam intensity) data}

To calculate the relative averaged bunch intensity losses, the measured bunch intensity $I$ is first normalized to the initial intensity $I_{0}$. The relative losses $I_{\text {lost }, j}$ for each individual bunch are then given by:

$$
I_{\text {lost }, \mathrm{j}}=1-\frac{I_{j}}{I_{j, 0}}, j=1, \ldots, 216
$$

The average is then taken over the bunches with the same excitation amplitude:

$$
I_{\text {lost }}(n \cdot \Delta A)=\frac{1}{6} \cdot \sum_{j=\text { bunches with } n \cdot \Delta A} I_{\text {lost }, \mathrm{j}} .
$$

in order to reduce the noise of the measurement. An example plot for the so obtained relative loss rate is shown in Fig. 7 for the $7^{\text {th }}$ turn pulsing.

For a quantitative comparison of the losses for the different maximum excitation amplitudes $A_{\max }$, we further define the average loss rate for each excitation amplitude $n \cdot \Delta A$

$$
L_{\mathrm{loss}}(n \cdot \Delta A):=<\frac{I_{\text {start excitation }}-I_{\text {end excitation }}}{I_{\text {start excitation }} \cdot \Delta t_{\text {excitation }}}>_{\text {bunches with } n \cdot \Delta A}
$$

The average is here taken over the bunches with the same excitation amplitude $n \cdot \Delta A$. As the duration of the excitations differs for the different maximum excitation amplitudes $A_{\max }$, it is better to compare loss rates instead of relative losses. An example for the comparison of the so obtained loss rates is shown in Fig. 8 for the $7^{\text {th }}$ turn pulsing.

\section{B. BWS (wire scanner) data}

During the relevant fill 6220 there was most likely a bug in the logging. When the number of scanned slots was increased to the maximum number of 254 , the profile data was not logged any more and thus also emittances (Fig. 3) 

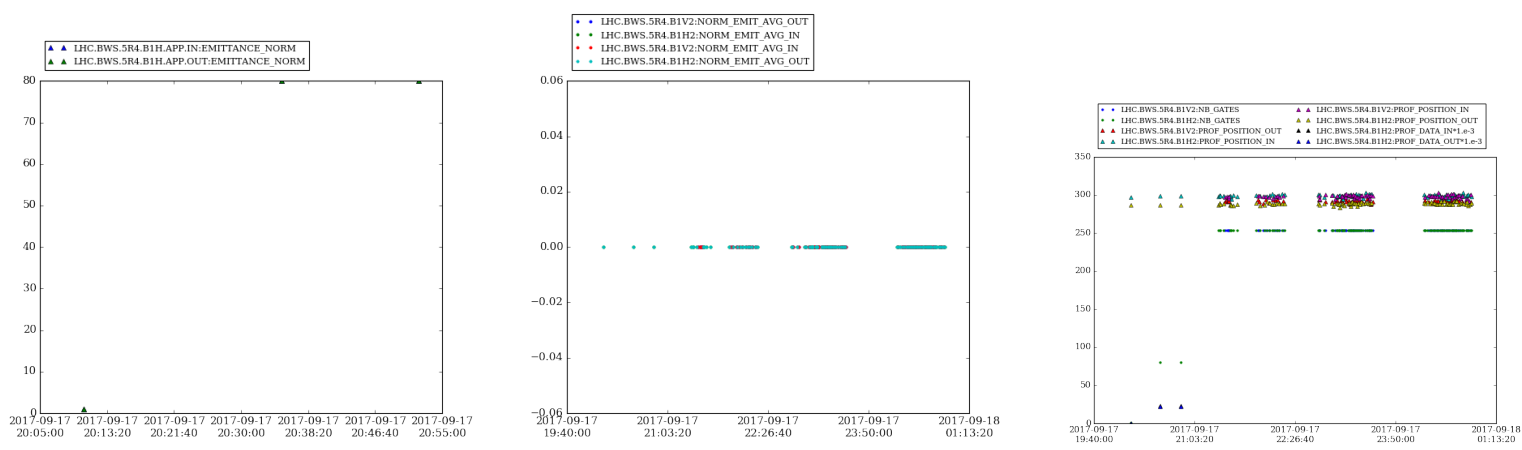

Figure 3. BWS data as extracted from timber. Due to an error in the logging, the relevant profile data was unfortunately not stored and with it also no emittance values.

\section{BSRT profiles at injection}

This chapter intends to give a short overview of the BSRT profile analysis presented in this note. For a more detailed description of the analysis, it is referred to [10]. The BSRT image formation is mathematically described as a convolution of the beam distribution with the optical resolution (LSF). Assuming that the beam distribution as well as the optical resolution (LSF) are Gaussian, a conversion factor $c_{\mathrm{LSF}, z}$ ("LSF factor") can be derived which is nothing else than the width of the Gaussian distribution of the optical resolution ${ }^{2}$ :

$$
\sigma_{\text {beam }, z}=\sqrt{\sigma_{\text {profile }, z}^{2}-c_{\mathrm{LSF}, z}^{2}}, z=h, v
$$

The position can then be very roughly also be expressed in beam sigma:

$$
z\left[\sigma_{\text {beam }, z}\right]=\frac{z[\mathrm{~mm}]}{\sigma_{\text {beam }, z}[\mathrm{~mm}]}, z=h, v .
$$

This "LSF factor" is stored in the LHC Logging database.

The BSRT profiles are in general a projection of the BSRT synchrotron light black and white image projected on the horizontal and vertical axis. The result are histograms in both planes representing the projections of the horizontal/vertical bunch distribution. The BSRT can take images of only one bunch at a time and in order to record all bunches during one fill the device loops through the individual bunches. Before several profiles of the same individual bunch were taken for each time stamp. This has been changed in this MD, and only one profile is taken per time stamp and bunch. A typical bunch distribution for a reference bunch without any excitation is shown in Fig. 4.

Using these profiles, the general steps in the analysis are:

\footnotetext{
2 The convolution of two uni-variate Gaussian distributions $\mathrm{f}$ and $\mathrm{g}$ having respectively the means $\mu_{f}$ and $\mu_{g}$ and standard deviation $\sigma_{f}$ and $\sigma_{g}$ is a Gaussian distribution with mean and standard deviation $\mu_{f * g}=\mu_{f}+\mu_{g}$ and $\sigma_{f * g}=\sqrt{\sigma_{f}^{2}+\sigma_{g}^{2}}$
} 


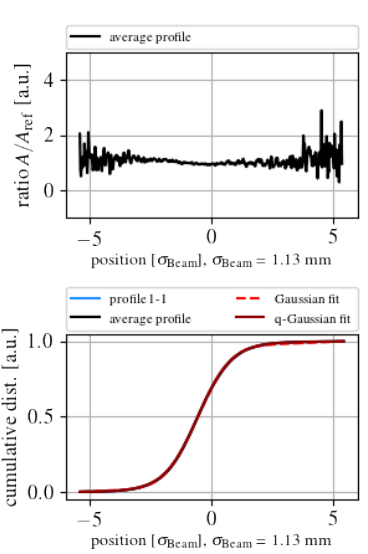
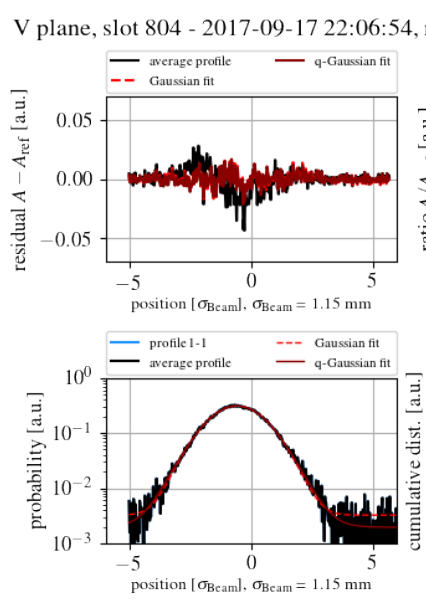


Figure 4. Typical BSRT profile at injection for the horizontal (left) and vertical (right) plane. The profile was taken towards the end of the $7^{\text {th }}$ turn excitation during fill 6220 (2017-09-17 22:06:54) and for a bunch not experiencing any excitation. For this bunch, the transverse damper is not active. Similar profiles are obtained also for the bunches with transverse damper active. The residual and ratio are taken in respect to a profile at the beginning of the fill (2017-09-17 21:21:08). The background is not subtracted and the moving average profiles are shown as black line in all subplots. For the moving average the profiles are explicitly averaged over 11 time stamps. Note that the $\mathrm{x}$-axis is the position of the BSRT image projection and not the proton beam sigma. The raw profiles for this time stamp are shown in blue (only one profile per time stamp and bunch) in the probability distribution (lower left) and cumulative sum (lower right). For the residual and ratio, the moving average profile is shown in black together with the $1 \sigma$ standard deviation over the 11 time stamps as gray envelope. The Gaussian fit is shown in dashed red and the q-Gaussian fit in solid dark red in the profile plot (lower left) and the deviation from the fit with the same color coding in the residual plot (upper left).

1. The profiles are converted to probability distributions $\rho(x)$ by dividing the bin height by the integral over the profile, so that:

$$
\int_{-\infty}^{\infty} \rho(x)=1
$$

2. No background subtraction is performed in this analysis as it did not prove to be useful.

3. The profiles are averaged in order to reduce the noise. As only one profile is taken per time stamp and bunch, the step of averaging over the profiles for each time stamp is omitted and directly the moving average and standard deviation over 11 time stamps ("moving average profile") taken. The obtained standard deviation for each bin is used as an estimate for the error in each bin. These estimated errors are then used as weights for the least square fit of the Gaussian and q-Gaussian distribution and for the calculation of the chi-squared as a measure of the goodness of the fit. The moving average profile is shown as a black line in Fig. 4.

4. A Gaussian (light red dashed line in Fig. 4) and q-Gaussian distribution (dark red line in Fig. 4) are fitted to the moving average profile and the fit is weighted with the calculated standard deviation for 
each bin.

The Gaussian distribution is defined as

$$
f_{\text {Gauss }}(x):=c+a \cdot \frac{e^{-\frac{(x-\mu)^{2}}{2 \sigma^{2}}}}{\sqrt{2 \pi} \sigma},
$$

where $\mu$ is the mean and $\sigma$ the standard deviation of the distribution.

The q-Gaussian distribution is defined as

$$
f_{\mathrm{q}-\text { Gauss }}(x)=c+a \cdot \frac{\sqrt{\beta}}{C_{q}} e_{q}\left(-\beta(x-\mu)^{2}\right),
$$

where $\mu$ is the mean of the distribution and

$$
e_{q}(x)=(1+(1-q) x)^{\frac{1}{1-q}}
$$

is the q-exponential. The normalization factor $C_{q}$ is given by

$$
C_{q}=\frac{\sqrt{\pi} \cdot \Gamma\left(\frac{3-q}{2(q-1)}\right)}{\sqrt{q-1} \cdot \Gamma\left(\frac{1}{q-1}\right)}, \text { for } 1<q<3,
$$

For $q<\frac{5}{3}$ the standard deviation $\sigma$ is then given by:

$$
\sigma^{2}=\frac{1}{\beta(5-3 q)}
$$

For other values of $q$ the standard deviation is either infinite or not defined. The range of the parameter $q$ is limited to $0<q<3$. The parameter $q$ is an estimator for the tail population. For $q \rightarrow 1$ the Gaussian distribution is recovered, for $q>1$ the distribution features heavier tails compared to the Gaussian distribution and for $q<1$ lighter tails. The parameter $c$ is introduced in the Gaussian and q-Gaussian fit in order to model the background of the profiles implying the introduction of the parameter $a$ in order to fulfill the requirement that the integral over the distribution is one.

5. statistical parameters are calculated for the average and moving average profile, including the cumulative sum of the distribution shown in Fig. 4 lower right.

6. To better visualize the changes of the distribution, the residual Res and the ratio Rat for bunch $i$ at time stamp $t_{i}$ in respect to reference bunch $j$ at time stamp $t_{j}$ are calculated with

$$
\begin{aligned}
\operatorname{Res}\left(t_{i}, x\right) & =A_{i}\left(t_{i}, x\right)-A_{j}\left(t_{j}, x\right) \\
\operatorname{Rat}\left(t_{i}, x\right) & =\frac{A_{i}\left(t_{i}, x\right)}{A_{j}\left(t_{j}, x\right)}
\end{aligned}
$$


where $A_{i}\left(t_{j}, x\right)$ denotes the amplitude at bin $x$ of bunch $i$ at time stamp $t_{j}$. In this MD the residual and ratio are taken in respect to the initial distribution of the bunch itself, explicitly:

$$
\begin{aligned}
\operatorname{Res}\left(t_{i}, x\right) & =A_{i}\left(t_{i}, x\right)-A_{i}\left(t_{j}, x\right) \\
\operatorname{Rat}\left(t_{i}, x\right) & =\frac{A_{i}\left(t_{i}, x\right)}{A_{i}\left(t_{j}, x\right)}
\end{aligned}
$$

The residual is in general sensitive to changes in the core region and the ratio to changes in the tails. The residual proved to be a good indicator for changes in the core while the ratio turned out to be too sensitive to the noise of the BSRT profiles.

The following observations can be made:

- The distribution in the horizontal plane features over-populated tails and the q-Gaussian fit represents a better model for the distribution. The tails are depleted during the fill as illustrated in Fig. 5 showing a decrease of $q$. The bunches with transverse damper active feature a higher $q$-parameter up to 1.4 while the ones with transverse damper active show less populated tails with a $q$-parameter starting from 1.25 .

- The distribution in the vertical plane is almost Gaussian. As in the horizontal plane, the bunches with transverse damper active feature a higher $q$-parameter up to 1.15 while the ones with transverse damper active show less populated tails with a $q$-parameter up to 1.1 .
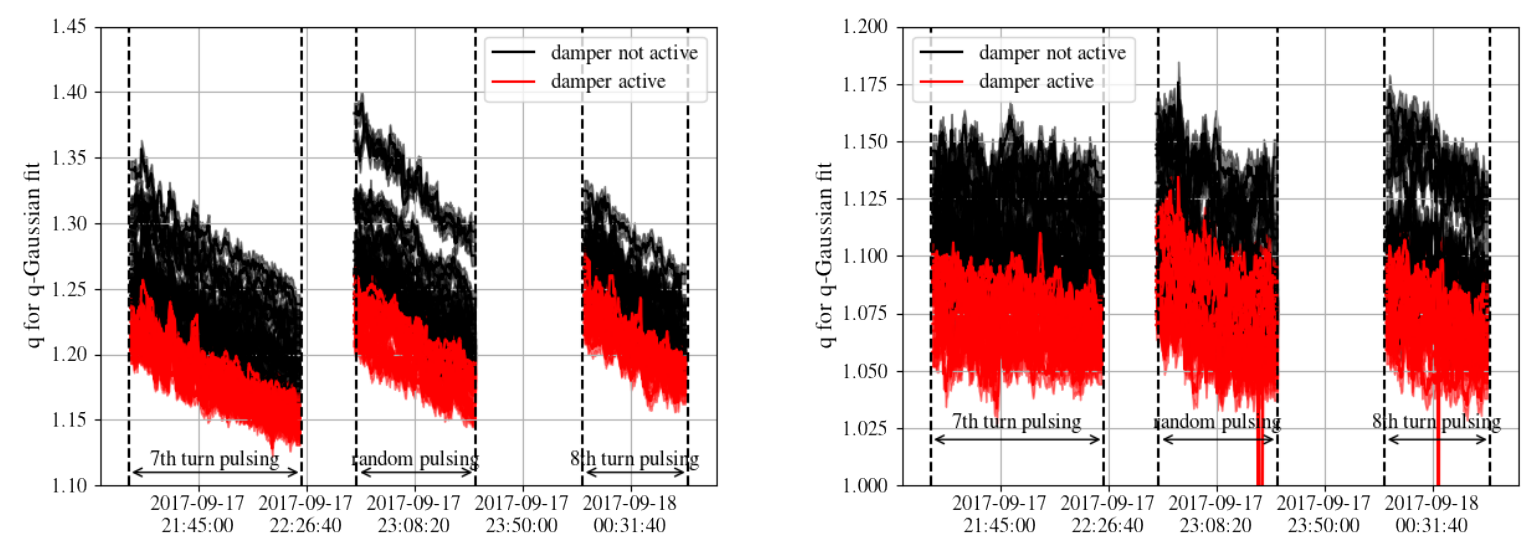

Figure 5. $q$ parameter of q-Gaussian fit in the horizontal (left) and vertical (right) plane: $q$-parameter for references bunches not experiencing any excitation during fill 6220. The bunches with transverse damper active are shown in red and the ones with the transverse damper not active in black. The q-Gaussian fit is performed for the moving average profiles and without background subtraction. The errorbars contain only the error from the q-Gaussian fit obtained from the covariance matrix $\left(\sigma_{q}=\sqrt{\left(\operatorname{cov}\left(p_{q}, p_{q}\right)\right.}\right.$ where $p_{q}$ indicates the diagonal element of the matrix for the fit parameter $q$ ). In the horizontal plane the tails are clearly overpopulated in respect to a Gaussian distribution as $q>1$ and slowly depleted during the fill visible as a decrease in $q$. In the vertical plane, the distribution is almost Gaussian as $q \approx 1$ and stay more or or less constant. 
- There are strong oscillations of the distribution during the fill mostly visible as fluctuation in the residual of the distribution. By averaging over several profiles as done in the moving average, the fluctuations can be reduced.

- The cumulative sum (CDF) of the distribution is a smooth function for both planes and can be used to define a model independent definition of the standard deviation $\sigma$ of the distribution ${ }^{3}$. Based on the analogy of the cumulative distribution function and the standard deviation for a Gaussian distribution we define the standard deviation $\sigma$ as:

$$
\begin{aligned}
& \sigma_{32}=\mathrm{CDF}^{-1}(0.32) \\
& \sigma_{68}=\mathrm{CDF}^{-1}(0.68)
\end{aligned}
$$

The left and right side are calculated separately because of the BSRT distribution is not always necessarily symmetric.

For the analysis of the statistical parameters obtained from the profiles the normalized profiles for each time stamps are used and in order to reduce the noise a moving average over 10 consecutive data points per bunch is taken denoted with \langle\rangle$_{10}$ in Eqn. 6. The obtained parameter $p$ is then averaged over the 6 bunches with the same excitation amplitude $n \cdot \Delta A$ denoted with \langle\rangle$_{\text {bunches with } n \cdot \Delta A}$ :

$$
p_{z}(n \cdot \Delta A)=\left\langle\left\langle p_{N, \text { bunch }, z}\right\rangle_{10}\right\rangle_{\text {bunches with } n \cdot \Delta A}, z=h, v \text {. }
$$



Figure 6. $7^{\text {th }}$ turn pulsing: emittance as measured with the BSRT (Gaussian fit) and as defined in Eqn. 22 for all 216 bunches (the 12 nominal and one pilot are not shown). The spikes are most likely due to the fit not convergence or too small amplitude of the profiles.

\footnotetext{
3 The BSRT emittance obtained from the LHC logging database (see Sec. III E 2) is the sigma of the distribution obtained via a Gaussian fit to the distribution assuming that a Gaussian fit is a well suited model. For example in the horizontal plane the tails are over-populated and the Gaussian distribution does not represent a good model.
} 
In addition, the relative growth rate of the parameter is also calculated:

$$
p_{N, z}(n \cdot \Delta A)=\left\langle\frac{\left\langle p_{N, \text { bunch }, z}\right\rangle_{10}}{\left\langle p_{N, \text { bunch }, z, 0}\right\rangle_{10}}\right\rangle_{\text {bunches with } n \cdot \Delta A}, z=h, v \text {. }
$$

Even after cleaning the data of any profiles considered to be faulty, the time evolution of almost all parameters still feature spikes or square like deviations if the moving average is taken. The deviations are based on a faulty measurement/profile and are not real physical effects. As example Fig. 6 shows the spikes present in the uncleaned BSRT emittance data from the LHC Logging database featuring the same behavior.

\section{BSRT emittance from the LHC Logging database}

In the LHC logging database, the $1 \sigma$ standard deviation $\sigma_{\text {profile, } z}$ obtained from a Gaussian fit to the horizontal and vertical projection of the BSRT profiles is stored. The sigma of the beam distribution $\sigma_{\text {bunch, } z}$ can then be calculated from $\sigma_{\text {profile }, z}$ and the correction factor from the system optical resolution $c_{\mathrm{LSF}, z}$ ("LSF factor"):

$$
\sigma_{\text {beam }, z}=\sqrt{\sigma_{\text {profile }, z}^{2}-c_{\mathrm{LSF}, z}^{2}}, z=h, v .
$$

From the beam sigma $\sigma_{\text {beam }, z}$ the normalized emittance can then be obtained via the well known formula:

$$
\varepsilon_{N, \text { bunch }, z}=\beta_{\text {rel }} \gamma_{\text {rel }} \frac{\sigma_{\text {beam }, z}^{2}}{\beta_{\mathrm{BSRT}, z}}, z=h, v,
$$

where $\beta_{\text {rel }}, \gamma_{\text {rel }}$ are the relativistic $\beta$ and $\gamma$, and $\beta_{\mathrm{BSRT}, z}$ is the beta function at the location of the BSRT as given in the LHC logging data base or as read in from an external file. For this MD the $\beta$-functions summarized in Table III have been used [11]. At injection, the main source of light is the undulator and therefore the values of the $\beta$-function at this location were used.

Table III. $\beta$-function values at the BSRT, explicitly the undulator MU.B5*, which is the main source of light at injection. The values are provided by the optics measurement team [11].

\begin{tabular}{|l|c|c|c|}
\hline Beam & Location & $\beta_{x}$ & $\beta_{y}$ \\
\hline Beam 1 & MU.B5R4 & $206.8 \pm 2.6$ & $287.3 \pm 3.8$ \\
\hline Beam 2 & MU.B5L4 & $193.1 \pm 2.6$ & $337.6 \pm 4.4$ \\
\hline
\end{tabular}

Similar to the parameter averaging in the BSRT profile analysis, we can define the emittance growth, relative emittance growth and emittance growth rate for each excitation amplitude $n \cdot \Delta A$. In order to reduce the noise, a moving average of the emittance over 10 consecutive data points per bunch is taken denoted with \langle\rangle$_{10}$ in Eqn. 24. The obtained emittance is then averaged over the 6 bunches with the same excitation amplitude $n \cdot \Delta A$ denoted with \langle\rangle$_{\text {bunches with } n \cdot \Delta A}$ :

$$
\varepsilon_{z}(n \cdot \Delta A)=\left\langle\left\langle\varepsilon_{N, \text { bunch }, z}\right\rangle_{10}\right\rangle_{\text {bunches with } n \cdot \Delta A}, z=h, v .
$$


In addition, the relative emittance growth is also calculated:

$$
\varepsilon_{N, z}(n \cdot \Delta A)=\left\langle\frac{\left\langle\varepsilon_{N, \text { bunch }, z}\right\rangle_{10}}{\left\langle\varepsilon_{N, \text { bunch }, z, 0}\right\rangle_{10}}\right\rangle_{\text {bunches with } n \cdot \Delta A}, z=h, v .
$$

As example the averaged and relative averaged emittance growth if illustrated in Appendix A, Fig. 37 and 39 for the $7^{\text {th }}$ turn pulsing.

Similar to the loss rate, we can also define the emittance growth rate:

$$
L_{\text {emit }, z}(n \cdot \Delta A):=<\frac{\varepsilon_{N, z, \text { start excitation }}-\varepsilon_{N, z, \text { end excitation }}}{\varepsilon_{N, z, \text { start excitation }} \cdot \Delta t_{\text {excitation }}}>_{\text {bunches with } n \cdot \Delta A}, z=h, v .
$$

where $\varepsilon_{N, z}, *$ excitation is the normalized emittance at the start/end of the excitation. The dependence of the emittance growth rate as obtained from the LHC Logging database on the excitation amplitude $n \cdot \Delta A$ and $A_{\max }$ is illustrated for example in Fig. 10 for the $7^{\text {th }}$ turn pulsing.

Due to the occurrence of faulty profiles during this MD, the Gaussian fit did not always converge. In this case the beam size is set to zero resulting in spikes in the emittance as shown in Fig. 6. To get rid of all non-convergent fits, all values with $\sigma_{\text {profile }, z}<0.8 \mathrm{~mm}$ are removed.

\section{E. Fill 6220, $7^{\text {th }}$ turn}

In the following sections the analysis of the FBCT data for losses (Sec. III E 1) and BSRT data for emittance (Sec. IIIE2) and profiles (Sec. IIIE3) is presented. The results are then compared with simulations (Sec. III E 4).

\section{Losses and loss rates from FBCT measurements}

Fig. 7 shows the relative bunch intensity losses measured with the FBCT for pulsing in $\mathrm{H}+\mathrm{V}$. The losses clearly depend on the excitation amplitude. The damper does not appear to have any effect. The losses caused by an excitation in the horizontal plane are sightly larger than the ones in the vertical plane (see Appendix A 1, Fig. 35 for pulsing in $\mathrm{H}, \mathrm{V}$ and $\mathrm{H}+\mathrm{V}$ ).

A quantitative comparison of the loss rate defined by Eqn. 4 for the two different maximum excitation amplitudes ( $A_{\max }=6 \mathrm{nrad}, A_{\max }=12 \mathrm{nrad}$ ) is shown in Fig. 8. As the duration of the excitations differs for the different maximum excitation amplitudes $A_{\max }$, it is better to compare loss rates instead of relative losses. The loss rate for one maximum excitation amplitude $A_{\max }$ in general scales quadratically with the excitation amplitude $n \cdot \Delta A, n=0, \ldots 5$ indicated by a quadratic fit in Fig. 8. Compared to the previous MD (see red and yellow line in Fig. 8), the losses increase quadratically while during this MD the losses appear to saturate for the second maximum amplitude $A_{\max }=12 \mathrm{nrad}$. The losses were also in general higher during the previous MD 1415 compared to this MD 2167. The source of this difference in behavior is not known, but one option could be the different tune, tune spread and optics. 

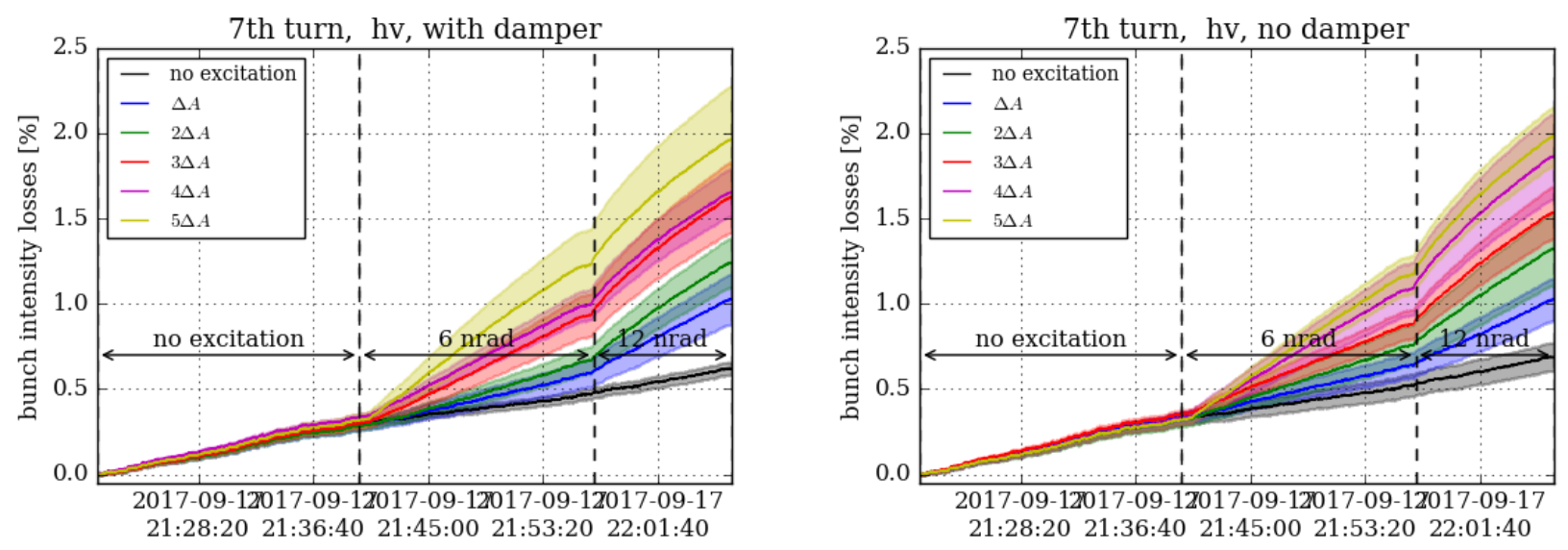

Figure 7. $7^{\text {th }}$ turn pulsing: Relative bunch intensity losses measured with the FBCTs (Eqn. 3). The average over the group of 6 bunches experiencing the same excitation amplitude is indicated by a solid line together with the $1 \sigma$ standard deviation over the four bunches indicated as an envelope in the same color. The maximum excitation amplitudes are indicated with black arrows. A clear increase of the losses with the excitation amplitude within the bunch train $(n \cdot \Delta A)$ and at each increase of the maximum amplitude $\left(A_{\max }\right)$ is visible. The damper does not appear to have any effect.
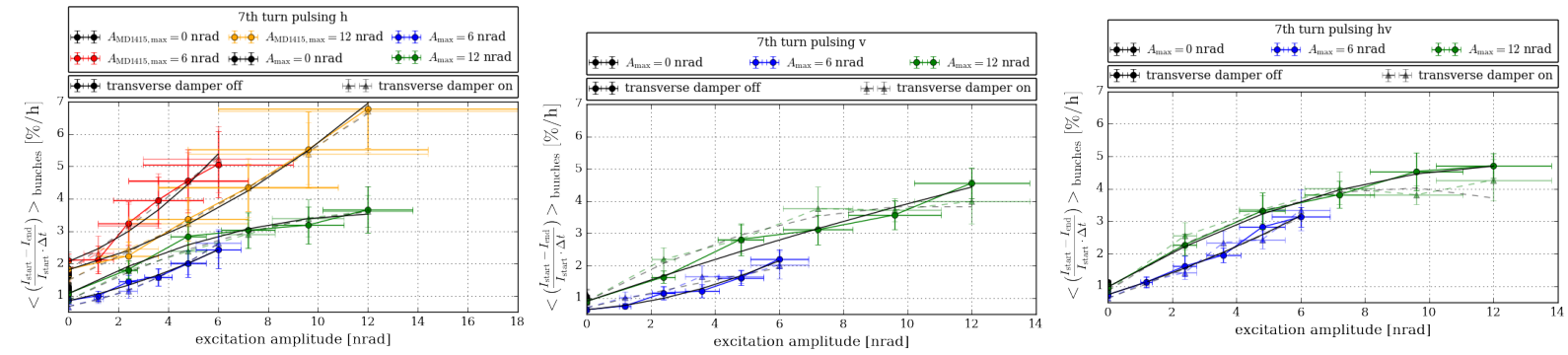

Figure 8. $7^{\text {th }}$ turn pulsing: Average bunch intensity loss rate measured with the FBCTs (Eqn. 4) during this MD 2167 (blue and green) and for the previous MD 1415 (red and yellow). The bunches for which the transverse damper is active are shown with solid dots and the bunches for which the transverse damper is not active are shown with slightly transparent triangles. Each point represent the average over the six bunches with the same excitation amplitude $n \cdot \Delta A$. The loss rates follow in general a quadratic behavior. The second order polynomial fit to the loss rate is indicated with a solid line for the bunches with transverse damper active and with a dashed line for the bunches with the transverse damper not active. The error on the excitation amplitude during the previous MD 1415 is estimated to be around 50\% and could be reduced in this MD to 10-15\%, where the upper limit of $15 \%$ has been assumed for this plot.

The difference in loss rates for equivalent $n \cdot \Delta A$ but different maximum amplitudes $A_{\max }$ can be explained by the following argument: The loss rate does not distinguish between initial losses due to an initial depletion of the tails occurring when the excitation is switched on and continuous losses due to diffusive processes caused by the excitation. As there is one injection per pattern, the beam has already been excited when increasing the excitation amplitude from the first $A_{\max }$ to the second. As the excitation changes the distribution, the initial distributions for the two different $A_{\max }$ are therefore not the same. 


\section{BSRT emittance from LHC logging database (Gaussian fit)}

For $7^{\text {th }}$ turn pulsing, the emittance features the following excitation amplitude behavior:

- There is no dependence of the emittance growth on the excitation amplitude in the horizontal plane.

- Excitation amplitude dependent emittance growth in the vertical plane is only observed for an excitation in $\mathrm{V}$ and $\mathrm{H}+\mathrm{V}$.

- In case of an excitation amplitude dependent emittance growth, the emittance increases rapidly over


Figure 9. $7^{\text {th }}$ turn pulsing: relative emittance in the horizontal plane (left) and vertical plane (right) for an excitation in $\mathrm{H}+\mathrm{V}$ and measured with the BSRT (Gaussian fit) and as defined in Eqn. 24 together with the $1 \sigma$ standard deviation over the bunches with the same excitation amplitude $n \cdot \Delta A$ indicated as an envelope for each amplitude. For all emittance values a moving average over 10 time stamps is performed.
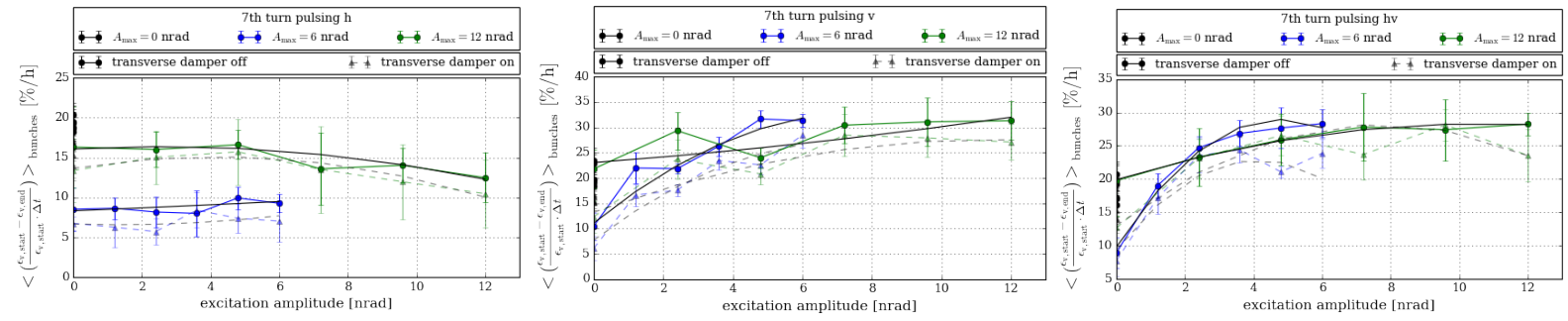

Figure 10. $7^{\text {th }}$ turn pulsing: Average emittance growth rate in the vertical plane measured with the BSRT and as defined in Eqn. 25 using the emittance $\varepsilon$ from the LHC Logging database. The measurements for the different maximum excitation amplitudes $A_{\max }$ are indicated in different colors. The bunches for which the transverse damper is active are shown with solid dots and the bunches for which the transverse damper is not active are shown with slightly transparent triangles. The emittance growth rate increases approximately quadratically with the excitation amplitude $n \cdot \Delta A$, if a dependence on the excitation amplitude is observed. The quadratic fit to the emittance growth rate is indicated with a solid line for the bunches with transverse damper active and with a dashed line for the bunches with the transverse damper not active. For all emittance values a moving average over 10 time stamps is performed. Emittance growth in only observed for an excitation in $\mathrm{V}$ and $\mathrm{H}+\mathrm{V}$. 
less than 3 minutes (keep in mind that the time scale is smeared by the moving average), which indicates a rapid adjustment of the beam distribution to a new stationary state.

As an example, the relative average emittance growth as defined by Eqn. 24 is shown in Fig. 9 for pulsing in only $\mathrm{H}+\mathrm{V}$ with the transverse damper not active showing no emittance growth in the horizontal plane, but an excitation amplitude dependent emittance growth in the vertical plane. The relative and absolute emittance for all excitation planes are summarized in Appendix A2, Figs. 36-39. The dependence of the vertical emittance growth rates on the excitation amplitude $n \cdot \Delta A$ and $A_{\max }$ is shown in Fig. 10 and for both planes in Appendix A 2, Fig. 40. As the vertical emittance increases rapidly during the first excitation, here $A_{\max }=6 \mathrm{nrad}$, and then reaches a kind of steady state, the emittance growth for the following excitation with the same bunches, here $A_{\max }=12 \mathrm{nrad}$, is much less. Therefore, for future measurements, one should consider actually measuring each excitation pattern and each maximum excitation amplitude $A_{\max }$ in a fresh fill in order to start from an as similar as possible initial distribution. The rapid emittance increase followed by a new constant increase actually agrees well with what is observed in simulations (see Sec. III E 4, Fig. 15 and [8]), where a sudden increase of the emittance due to a distribution change over the first 10000 turns is seen followed by a decrease of the emittance due to losses indicating that the distribution has reached a new equilibrium state with larger diffusion. This behavior is a very interesting phenomena of the non-linear dynamics and how it changes in general the beam distribution to a new equilibrium state.

\section{BSRT profiles}

The LHC Logging database provides only the statistical parameters obtained through a Gaussian fit to the BSRT profiles and thus assumes a model for the beam distribution. As shown in Sec. III C, the BSRT profiles are actually non-Gaussian and instead of fitting a mediocre model to the distribution, it is therefore better to use instead a model independent measure for the beam distribution. For this MD we chose to use the cumulative sum as it is in case of the BSRT profiles a smooth function of the position as shown for example in Fig. 13. The beam sigma calculated over the cumulative sum (Fig. 11 as example and Appendix A 3d, Figs. 41-42 for all plots) features a similar behavior as the emittance calculated with a Gaussian fit (Fig. 9 as example and Appendix A 2, Figs. 36-37 for all plots) and thus confirms the observations made in Sec. III E 2. As a cross check for the orbit stability, the centroid calculated with the cumulative sum is shown in Appendix A 3 d, Fig. 43-44, which stays constant during the entire excitation period.

As alternative to the Gaussian fit and a better model for a Gaussian like distribution but with over- (or under-) populated tails, is the q-Gaussian fit as introduced in Sec. IIIC. The fit is applied to the moving average profiles. Applying this model, an excitation amplitude dependent effect is observed only in the vertical plane and for pulsing in $\mathrm{V}$ and $\mathrm{H}+\mathrm{V}$, explicitly:

- increase of the standard deviation with the excitation amplitude $n \cdot \Delta A$ indicating a general emittance growth (as an example Fig. 12 (top) and Appendix A 3 c,Fig. 51 for all cases) 

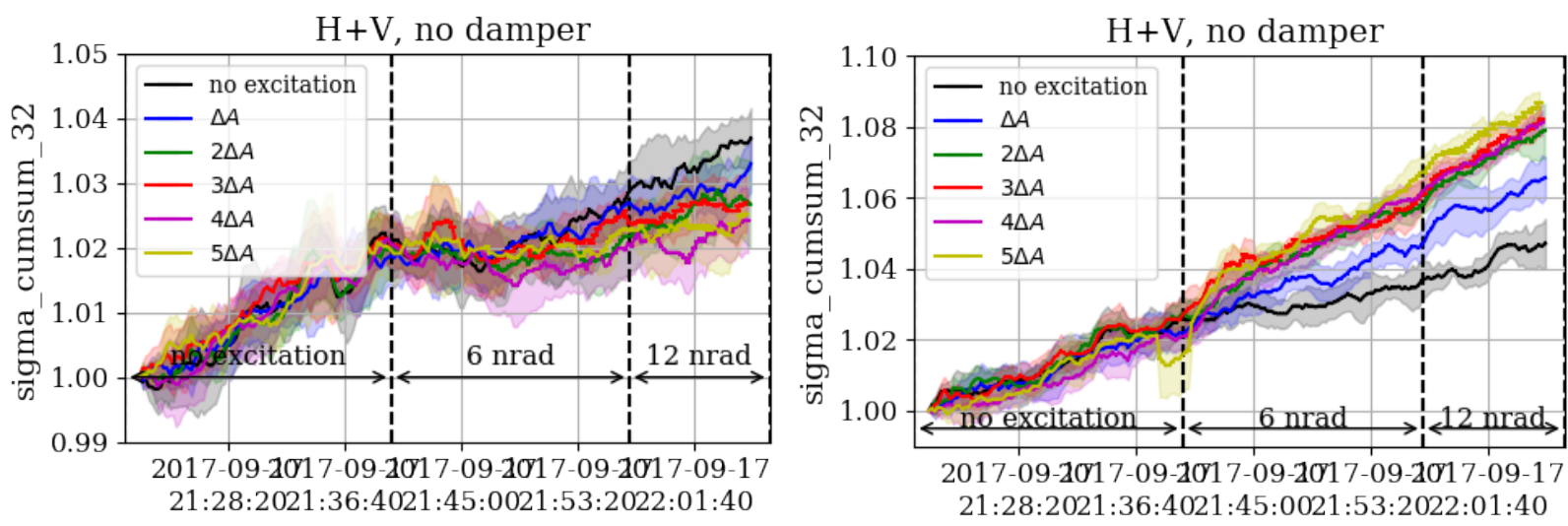

Figure 11. $7^{\text {th }}$ turn pulsing: relative beam sigma in the horizontal (left) and vertical (right) plane calculated over the cumulative sum (Eqn. 17) and measured with the BSRT together with the $1 \sigma$ standard deviation over the bunches with the same excitation amplitude $n \cdot \Delta A$ indicated as an envelope for each amplitude. For all beam sigma values a moving average over 10 time stamps is performed and the average, explicitly not moving average, profiles are used. A clear excitation amplitude dependent emittance growth in the vertical plane is visible as also observed in the BSRT emittance extracted from the LHC Logging database (Fig. 9).

- increase of the fit parameter $q$ with the excitation amplitude $n \cdot \Delta A$ indicating heavier tails in the distribution (as an example Fig. 12 (center) and Appendix A 3 c,Figs. 52-53 for all cases)

- decrease of the fit parameters $a$ and $c$ with the excitation amplitude $n \cdot \Delta A$ indicating a depletion of the high amplitude tails (as an example Fig. 12 (bottom) and Appendix A 3 c,Figs. 54-55 for all cases).

In the horizontal plane, no strong dependence on the excitation amplitude is observed, but the following generally interesting observations on the evolution of the beam distribution can be made:

- slight excitation amplitude dependent decrease of the beam sigma and fit parameter $q$ suggesting a depletion of the core and lower amplitude tails (as an example Fig. 12 (top and center), Appendix A 3 b, Figs. 45-47 for all cases).

- increase of the fit parameters $a$ and $c$ indicating a stronger population of the high amplitude tails (as an example Fig. 12 (bottom), Appendix A 3 b, Figs. 48-49)

As the normalized $\chi^{2}$ also stays constant during the entire excitation period (Appendix A 3 b, Fig. 50 and Appendix A 3 c, Fig. 56), an artificial effect due to a change in the quality of the fit can be excluded.

The change in the vertical distribution is so pronounced that it can also be directly observed on the residual and ratio of the profile. As an example Fig. 13 shows the vertical BSRT profiles at the end of the $7^{\text {th }}$ turn excitation in $\mathrm{V}$ an $\mathrm{H}+\mathrm{V}$, one bunch without excitation as reference and one which has experienced the maximum excitation $5 \cdot \Delta A$. The profiles of one representative bunch per case are summarized in Appendix A $3 \mathrm{~d}$, Figs. 57-62. The change of the residual and ratio suggest:

- Increase of the distribution around $2.5-3.5 \sigma$. 
- Depletion of the beam core.

Note that the integral of the distribution is normalized to 1 , so an increase of the population at a certain amplitude always entails a decrease at another amplitude.
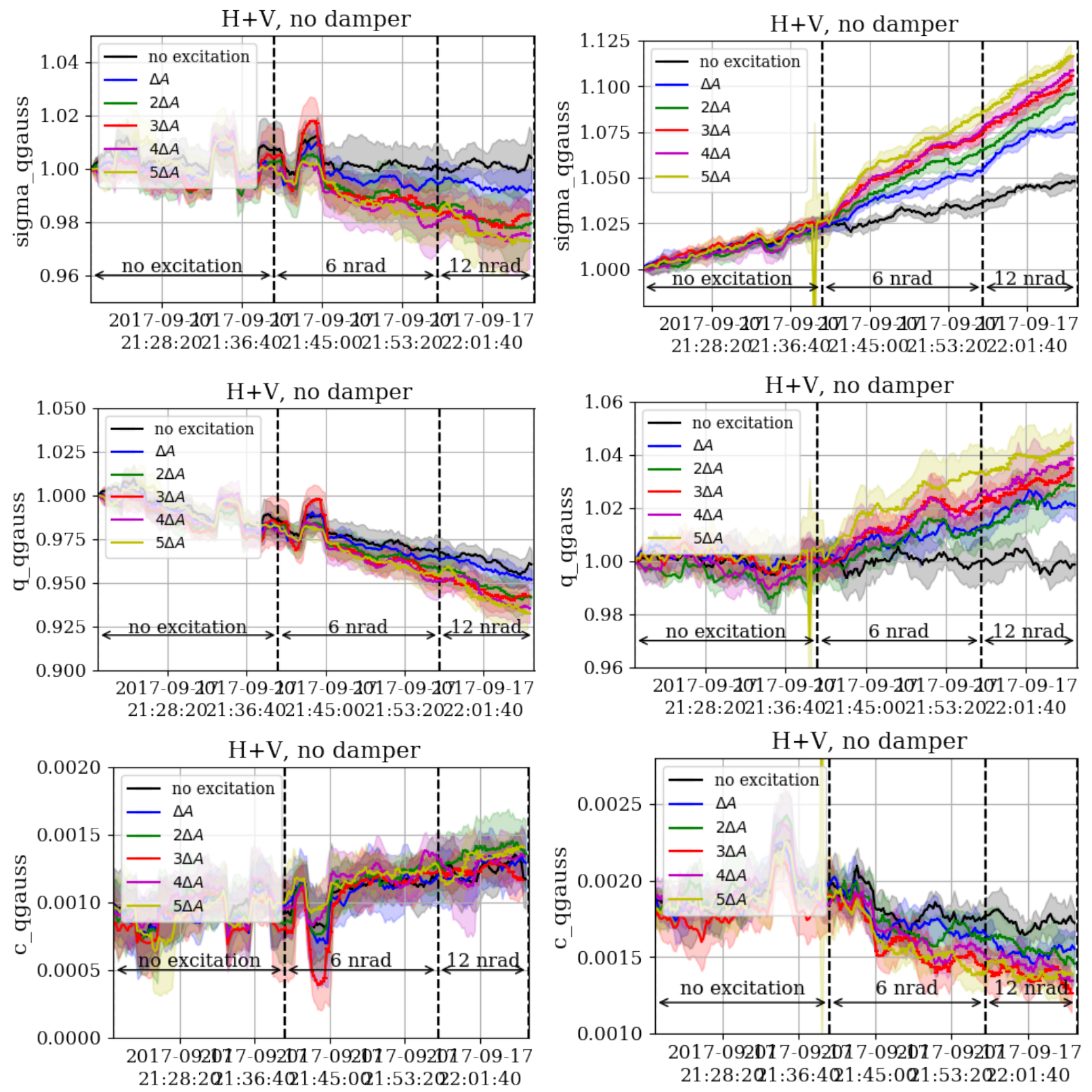

Figure 12. $7^{\text {th }}$ turn pulsing: relative beam sigma (top), relative fit parameter $q$ (center) and absolute value of the fit parameter $c$ from the q-Gaussian fit of the BSRT profiles (Eqn. 9) in the horizontal (left) and vertical (right) plane measured with the BSRT together with the $1 \sigma$ standard deviation over the bunches with the same excitation amplitude $n \cdot \Delta A$ indicated as an envelope for each amplitude. For all values the moving average profiles are used without any further averaging afterwards. 
no damper, no excitation

no damper, no excitation

V plane, slot 2666 - 2017-09-17 22:02:59, ref slot 2666 - 2017-09-17 21:35:00
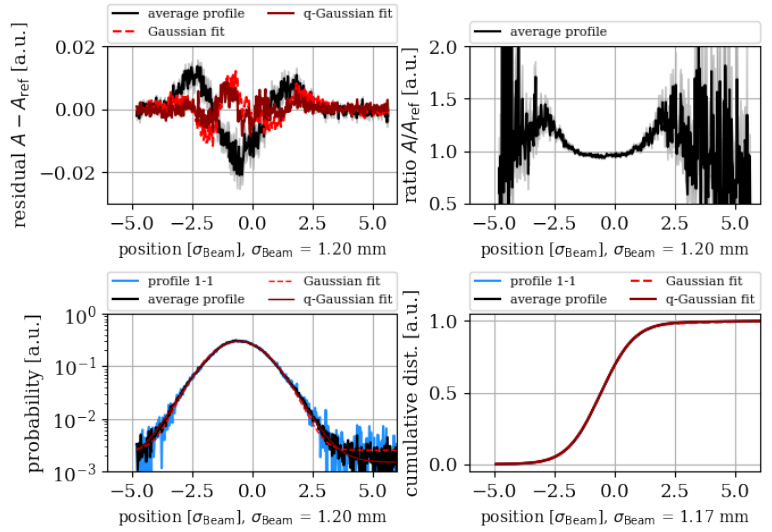

no damper, $5 \cdot \Delta A$ in $\mathrm{V}$

V plane, slot 1532 - 2017-09-17 22:02:55, ref slot 1532 - 2017-09-17 21:35:06
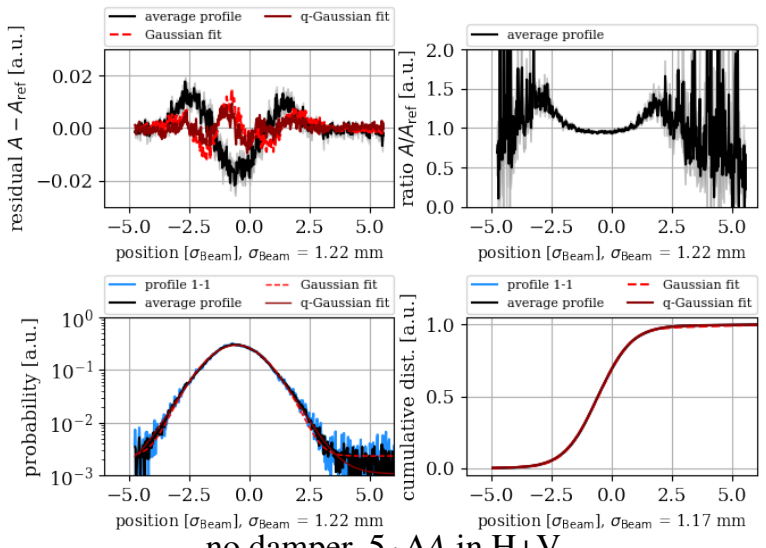
no damper, $5 \cdot \Delta A$ in $\mathrm{H}+\mathrm{V}$

V plane, slot 2696 - 2017-09-17 22:02:59, ref slot 2696 - 2017-09-17 21:35:00


Figure 13. $7^{\text {th }}$ turn pulsing: BSRT profile in the vertical plane of one bunch without resonant excitation (left) and one bunch experiencing the maximum excitation $\mathrm{V}$ (center) and in $\mathrm{H}+\mathrm{V}$ (right). The reference and excited bunch are taken out of the same group of 72 bunches. For all bunches the damper is not active. The residual is taken in respect to the start (2017-09-17 21:35) and end (2017-09-17 22:02) of the $7^{\text {th }}$ turn pulsing with a few minutes subtracted in order to account for the moving average of the profiles over 11 time stamps. The $\mathrm{x}$-axis is the position of the BSRT image projection and not the proton beam sigma.

\section{Comparison with simulations}

For the better understanding of the experimental results, an FMA analysis has been performed in order to identify the driving resonances. To obtain the dependence of the loss rate and emittance growth on the excitation plane and amplitude, tracking of a 6D Gaussian distribution over $10^{6}$ turns has been used. The simulations have been performed with Lifetrac [12] and the parameters are summarized in Table IV. In the distribution tracking, the orbit errors are disabled due to different implementation of the $a_{1}, b_{1}$ errors in Lifetrac and MAD-X.

In 2017 the machine tune was changed from $(64.28,59.31)$ to $(62.27,60.295)$ in standard operation accompanied by a slight change in optics. This change in tune entailed a change in the driving resonances, from previously the $7 Q_{x}$ resonances in 2016 [13] to the $7 Q_{x}, 7 Q_{y}$ and $7 Q_{x}+7 Q_{y}$ resonance in 2017 and also 
Table IV. Summary of simulation parameters for the distribution tracking with Lifetrac [12]. For details see [13] for 2016 simulations. The 2017 simulations use a similar setup except that a weighted Gaussian distribution is used in order to be more sensitive to losses of high amplitude particles and the larger emittance of $3.5 \mu \mathrm{m}$.

\begin{tabular}{|c|c|c|}
\hline Parameter & Distribution Tracking & FMA Analysis \\
\hline beam & \multicolumn{2}{|c|}{ Beam 1} \\
\hline beam energy & \multicolumn{2}{|c|}{$450 \mathrm{GeV}$} \\
\hline normalized emittance & $3.5 \mu \mathrm{m}$ & $2.5 \mu \mathrm{m}$ \\
\hline $4 \sigma$ bunch length & \multicolumn{2}{|c|}{$1.3 \mathrm{~ns}$} \\
\hline $1 \sigma$ bunch length & \multicolumn{2}{|c|}{$9.7 \mathrm{~cm}$} \\
\hline particle distribution & $\begin{array}{l}\text { 6D Gaussian distribution } \\
\qquad\left(10^{4} \text { particles }\right)\end{array}$ & $\begin{array}{l}\text { equally spaced grid in } x, y \\
\text { up to } 10 \sigma \text { with } \frac{\Delta p}{p_{0}}=0\end{array}$ \\
\hline turns tracked & $10^{6}$ turns & $10^{4}$ turns \\
\hline optics & \multicolumn{2}{|c|}{2017 injection optics, $\beta^{*}=11 \mathrm{~m}$} \\
\hline machine imperfections & $\begin{array}{c}\text { standard errors } \\
\text { with } a_{1}=b_{1}=0^{\text {a }}\end{array}$ & no errors \\
\hline octupoles & \multicolumn{2}{|c|}{$I_{\mathrm{MOF}}=+19.6 \mathrm{~A}, I_{\mathrm{MOD}}=-19.6 \mathrm{~A}$} \\
\hline tune $\left(Q_{x}, Q_{y}\right)$ & \multicolumn{2}{|c|}{$(62.27,60.295)$} \\
\hline chromaticity $\left(Q_{x}^{\prime}, Q_{y}^{\prime}\right)$ & \multicolumn{2}{|c|}{$(+15,+15)$} \\
\hline beam-beam & \multicolumn{2}{|c|}{ no collisions } \\
\hline transv. aperture ${ }^{\mathrm{b}}$ & $5.7 \sigma$ & - \\
\hline long. aperture & $10 \sigma$ & - \\
\hline $\begin{array}{l}7^{\text {th }} \text { turn pulsing at } \\
\text { ADT modules (sum over } 4 \text { modules) }\end{array}$ & 0,24 and $48 \mathrm{nrad}$ & $96 \mathrm{nrad}$ \\
\hline random noise at $\mathrm{ADT}$ & $6 \mathrm{nrad}$ & - \\
\hline
\end{tabular}

${ }^{\text {a }}$ Orbit errors are disabled due to different implementation of the $a_{1}, b_{1}$ errors in Lifetrac and MAD-X. $b_{2}$ errors are adjusted to yield an average (over 60 seeds) peak $\beta$-beat of $15 \%$ as expected from optics measurements in the LHC.

${ }^{\mathrm{b}} \sigma$ calculated for $3.5 \mu \mathrm{m}$ normalized emittance

a change in the affected amplitudes of the beam distribution (see Fig. 14 for the FMA analysis in frequency space and Appendix A 4, Fig. 63 in amplitude space).

The distribution tracking for $n^{\text {th }}$ turn pulsing usually features a fast adjustment over the first $10^{4}$ turns of the emittance to a new constant value meaning that the distribution adjusts and then reaches a new equilibrium state [13]. This behavior can be changed to an excitation amplitude dependent emittance growth by adding a random dipole noise component with uniform distribution. As a first estimate the value obtained in $[14,15]$ for $6.5 \mathrm{TeV}$ was used and scaled by the magnetic rigidity to the injection energy of $450 \mathrm{GeV}$, as no noise estimate is available at injection. This yields a maximum kick amplitude at the transverse damper of approximately

$$
\theta_{\text {random, ADT,max }}(450 \mathrm{GeV})=6 \mathrm{nrad}
$$





Figure 14. FMA analysis in frequency space for $7^{\text {th }}$ turn pulsing based on the 2017 injection optics with no machine errors and a tune of $(62.27,60.295)$. The excitation is $96 \mathrm{nrad}$ in each/both planes. The $7 Q_{x}$ and $7 Q_{y}$ resonance are both excited and in addition the 14 th order $7 Q_{x}+7 Q_{y}$.

which is most likely more an upper limit for the dipole noise to be expected at injection. Adding this random noise component, the simulations then yield correctly an excitation amplitude dependent emittance growth in the vertical plane only after a first fast adjustment of the beam distribution visible as an increased initial value. The so obtained simulations results are shown in Fig. 15. In general, higher excitation amplitudes compared to the experimental values have been used for simulations to see a noticeable effect.

In summary, the measurements showed strong excitation amplitude dependent losses for an excitation in $\mathrm{H}, \mathrm{V}$ and $\mathrm{H}+\mathrm{V}$ and emittance growth in the vertical plane for an excitation in $\mathrm{V}$ and $\mathrm{H}+\mathrm{V}$. In the horizontal plane no excitation amplitude dependent emittance growth was observed. In simulations, emittance growth in the vertical plane is observed for an excitation in $\mathrm{V}$ an $\mathrm{H}+\mathrm{V}$ and in the horizontal plane for an 



Figure 15. Relative intensity (top left) and relative vertical emittance (top right), and horizontal (bottom left) and vertical (bottom right) emittance obtained from simulations (Distribution Tracking) based on the 2016 injection optics with standard errors and $\left(Q_{x}, Q_{y}\right)=(64.28,59.31)$ and 2017 injection optics with standard errors and $\left(Q_{x}, Q_{y}\right)=$ $(62.27,60.295)$. The solid line indicates an excitation with only a random dipole noise component in $\mathrm{H}+\mathrm{V}$ of $6 \mathrm{nrad}$, and the dotted and dashed line the results for $7^{\text {th }}$ turn pulsing plus the random dipole noise component in $\mathrm{H}+\mathrm{V}$ of 6 nrad.

excitation in $\mathrm{H}+\mathrm{V}$. However higher losses are observed for an excitation in $\mathrm{H}$ and $\mathrm{H}+\mathrm{V}$ in simulations while in the experiment, the loss rates are independent of the excitation plane. Considering the complexity of the simulations and the starting point as presented in [13], the agreement of simulations and experiments has considerably improved by adding a random dipole noise component. However, the agreement is still far away from being able to make reliable predictions, one reason being that the LHC inherent noise has a non-negligible influence on the simulation results and is not well known, and also other effects influencing the complex diffusion process like electron cloud and intrabeam-scattering, both not included in the current simulation setup. 


\section{F. Fill 6220, uniform random excitation}

After the $7^{\text {th }}$ turn excitation, the beam was dumped and reinjected to try a random modulation. The random modulation is a uniform random distribution where all kick amplitudes are within $\left[0, A_{\text {ran,max }}\right]$, explicitly:

$$
A(\text { turn })=\operatorname{ran}(0,1)(\text { turn }) \cdot A_{\text {ran, } \max }
$$

with $\operatorname{ran}(0,1)$ a uniformly distributed random number between $[0,1]$, which is changed every turn. Consistent with the definitions and pattern for $n^{\text {th }}$ turn pulsing, the maximum amplitude of the random excitation $A_{\text {ran,max }}=n \cdot \Delta A$ is then changed according to the excitation pattern in Fig. 2. $A_{\max }=5 \cdot \Delta A$ defines the maximum excitation amplitude over the bunches, same as for the $n^{\text {th }}$ turn pulsing.

In the following sections the analysis of the FBCT data for losses (Sec. IIIF 1) and BSRT data for emittance (Sec. IIIF 2) and profiles (Sec. IIIF 3) is presented. The results are then compared with simulations (Sec. III F 4). Just before starting the random excitation a large orbit shift in the vertical plane occurred, the horizontal plane stayed stable (Fig. 16 and Appendix B 3 a, Figs. 72-73 for all bunches). Any change in losses, emittance or other beam parameters around this time stamp can therefore be attributed to this orbit shift with high probability.


Figure 16. random excitation: centroid calculated over the cumulative sum (Eqn. 17) in the horizontal (left) and vertical (right) plane measured with the BSRT together with the $1 \sigma$ standard deviation over the bunches with the same excitation amplitude $n \cdot \Delta A$ indicated as an envelope for each amplitude. No moving average over the profiles nor parameter is taken in order to show the un-smoothed oscillations. Just before switching on the random excitation a large orbit shift in the vertical plane occurred.

\section{Losses and loss rates from FBCT measurements}

As example Figs. 17-18 and Appendix B 1, Fig. 64 for all cases show the relative bunch intensity losses measured with the FBCT averaged over the bunches with the same excitation amplitude as defined in Eqn. 3. 
The losses clearly depend on the excitation amplitude. For $1 \mathrm{nrad}$ and $6 \mathrm{nrad}$ the losses are small and then increase dramatically for $12 \mathrm{nrad}$ maximum excitation amplitude. The losses for an excitation in the horizontal and vertical plane are considerably higher than for an excitation in only one of the planes. Both individual planes show similar losses. With the transverse damper active, the losses decrease considerably.
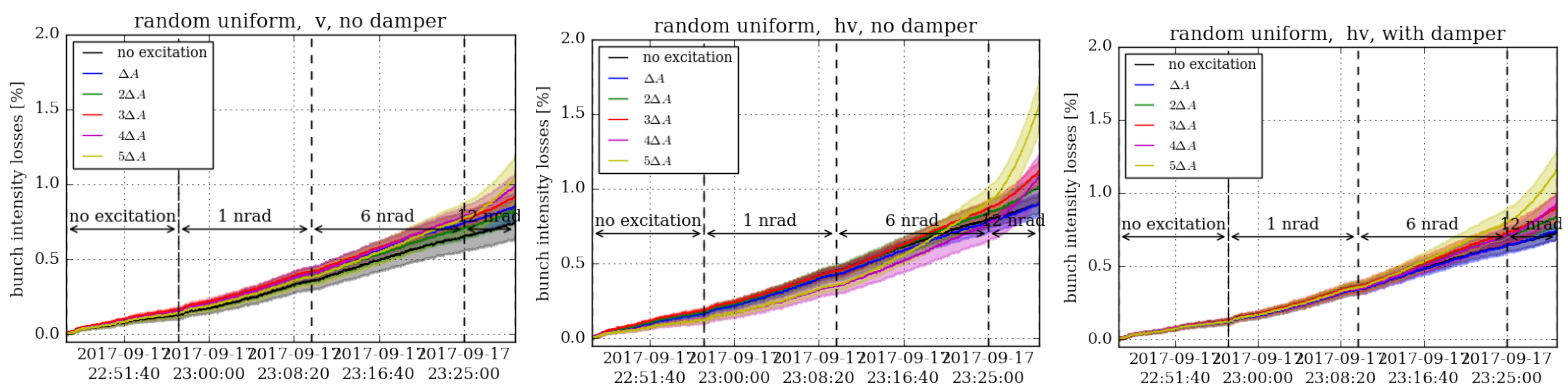

Figure 17. random uniform excitation: Relative bunch intensity losses measured with the FBCTs (Eqn. 3) for an excitation only in $\mathrm{V}$ with transverse damper not active (left), in $\mathrm{H}+\mathrm{V}$ with transverse damper not active (center) and in $\mathrm{H}+\mathrm{V}$ with transverse damper active (right). The average over the group of 6 bunches experiencing the same excitation amplitude is indicated by a solid line together with the $1 \sigma$ standard deviation over the four bunches indicated as an envelope in the same color. The maximum excitation amplitudes are indicated with black arrows. The losses caused by an excitation in the horizontal plane are similar to the ones in the vertical plane and appear to add up resulting in approximately double the losses in case of an excitation in the horizontal and vertical. The losses rate is also considerably reduced if the transverse damper is active.
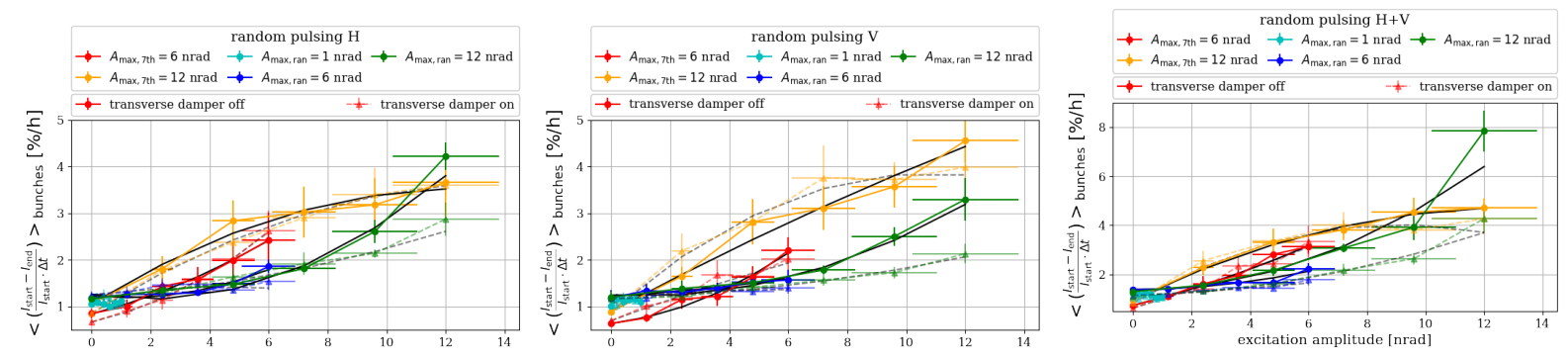

Figure 18. random uniform excitation: Average bunch intensity loss rate for an excitation in $\mathrm{H}$ (left), $\mathrm{V}$ (center) and $\mathrm{H}+\mathrm{V}$ (right) measured with the FBCTs (Eqn. 4) for a uniform random excitation (cyan, blue and green) and for $7^{\text {th }}$ turn pulsing during this MD (red and yellow). The bunches for which the transverse damper is active are shown with solid dots and the bunches for which the transverse damper is not active are shown with slightly transparent triangles. Each point represent the average over the six bunches with the same excitation amplitude $n \cdot \Delta A$. The second order polynomial fit to the loss rate is indicated with a solid line for the bunches with transverse damper active and with a dashed line for the bunches with the transverse damper not active. The error on the excitation amplitude during this MD is approximately to $10-15 \%$, where the upper limit of $15 \%$ has been assumed for this plot.

A quantitative comparison of the loss rate for random excitation and $7^{\text {th }}$ turn pulsing is shown in Fig. 18. The loss rates for both pulsing patterns follow a quadratic behavior indicated by a second order polynomial fit. The loss rates for $A_{\text {ran,max }}=12 \mathrm{nrad}$ are comparable for the $7^{\text {th }}$ turn pulsing and random excitation with a different sensitivity to the excitation plane, while for $A_{\text {ran,max }}=6$ nrad the loss rates for the random 
excitation are smaller than for the $7^{\text {th }}$ turn pulsing. Furthermore, this quantitative comparison also illustrates how the transverse damper reduces the loss rate in case of the random excitation while for the $7^{\text {th }}$ turn pulsing it does not have any effect.

\section{BSRT emittance from LHC logging database (Gaussian fit)}

For a random excitation, the emittance features the following excitation amplitude behavior:

- Emittance growth is only observed in the plane of excitation. This is illustrated in Fig. 19 on the example of pulsing in $\mathrm{H}, \mathrm{V}$ and $\mathrm{H}+\mathrm{V}$ with transverse damper not active and in Fig. 20 by comparing the emittance growth rates.
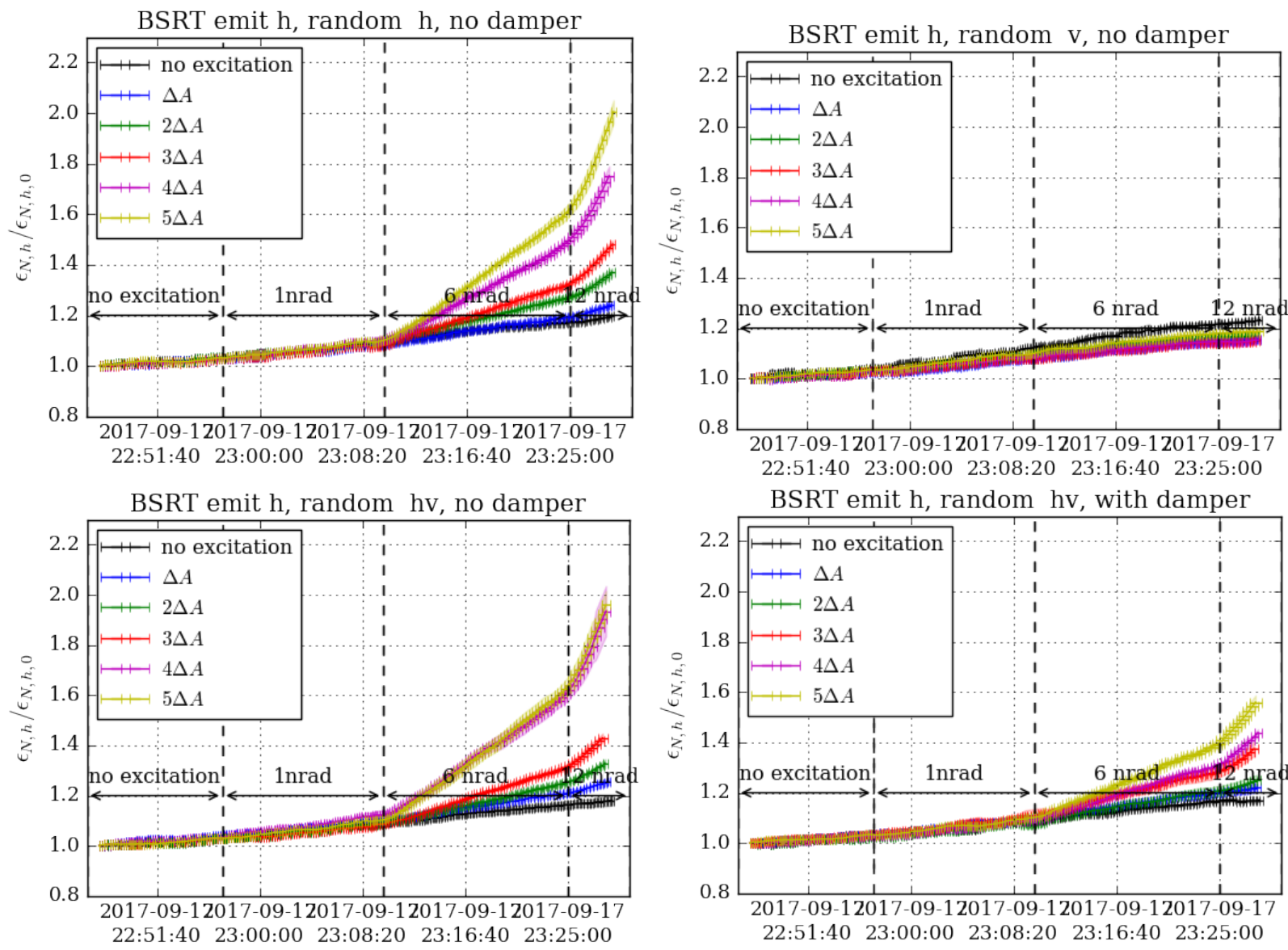

Figure 19. random uniform excitation: relative emittance in the horizontal plane for an excitation in $\mathrm{H}$ with transverse damper not active (upper left), $\mathrm{V}$ with transverse damper not active (upper right), $\mathrm{H}+\mathrm{V}$ with transverse damper not active (lower left) and $\mathrm{H}+\mathrm{V}$ with transverse damper active (lower right) measured with the BSRT (Gaussian fit) and as defined in Eqn. 24 together with the $1 \sigma$ standard deviation over the bunches with the same excitation amplitude $n \cdot \Delta A$ indicated as an envelope for each amplitude. For all emittance values a moving average over 10 time stamps is performed. The amplitudes $5 \cdot \Delta A$ and $4 \cdot \Delta A$ overlap for excitation in $\mathrm{H}+\mathrm{V}$. One reason for the behavior could be that the emittance for $4 \cdot \Delta A$ is much smaller than for $5 \cdot \Delta A$ (see Appendix B 2, Figs. 67-68) 

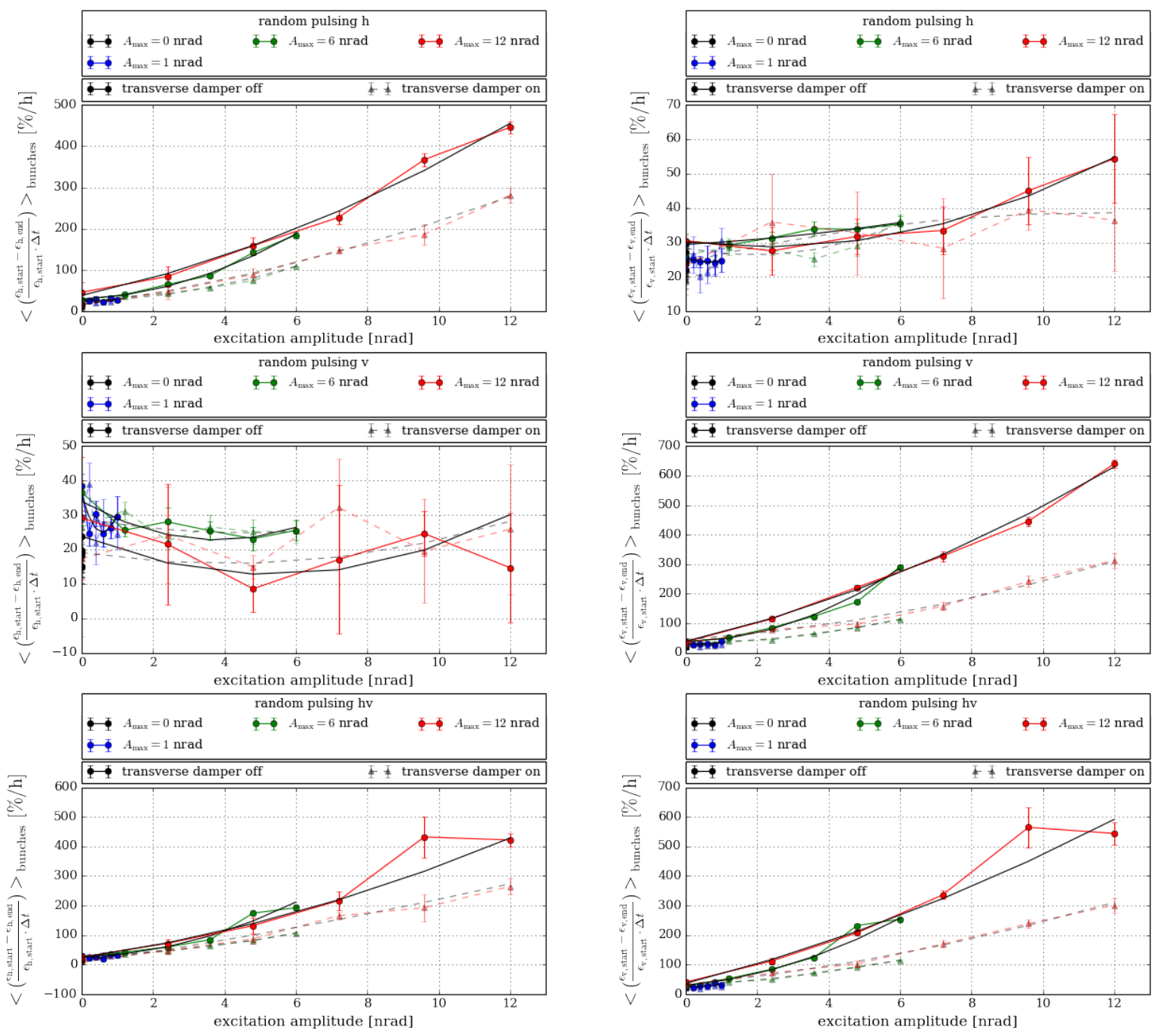

Figure 20. random excitation: Average emittance growth rate in the horizontal (left) and vertical plane (right) measured with the BSRT and as defined in Eqn. 25 using the emittance $\varepsilon$ from the LHC Logging database. The measurements for the different maximum excitation amplitudes $A_{\max }$ are indicated in different colors. The bunches for which the transverse damper is active are shown with solid dots and the bunches for which the transverse damper is not active are shown with slightly transparent triangles. The quadratic fit to the emittance growth rate is indicated with a solid line for the bunches with transverse damper active and with a dashed line for the bunches with the transverse damper not active. For all emittance values a moving average over 10 time stamps is performed.

- The emittance growth for exciting in one plane or in both is similar. This is illustrated in Fig. 19 on the example of pulsing in $\mathrm{H}$ and $\mathrm{H}+\mathrm{V}$ with transverse damper not active and in Fig. 20 by comparing the emittance growth rates.

- The emittance growth is more pronounced in the vertical plane (Fig. 20 and Appendix B 2, Figs. 6566).

- The emittance growth rate stays constant for a fixed excitation amplitude and during the entire du- 
ration of the excitation. This is in contrast to the $7^{\text {th }}$ turn pulsing, in which case the emittance first increases rapidly and then reaches a new steady state growth rate. This is illustrated in Fig. 19 for all cases where emittance growth is observed.

- The emittance growth is considerably reduced by the transverse damper. This is illustrated in Fig. 19 on the example of pulsing in $\mathrm{H}+\mathrm{V}$ with transverse damper not active and active.

- If emittance growth is observed, the growth rate increases approximately quadratically with the excitation amplitude (Fig. 20)

To compare all cases, the relative and absolute emittance for all excitation planes are summarized in Appendix B 2, Figs. 65-68. Comparing the emittance growth rates, an excitation only in $\mathrm{H}$ also results in a small emittance growth in $\mathrm{V}$, which is most likely due to coupling. This is not the case for an excitation only in $\mathrm{V}$ resulting in no emittance growth in $\mathrm{H}$ (Fig. 20).

\section{BSRT profiles}

Same as for the $7^{\text {th }}$ turn pulsing, we can use the beam sigma calculated over the cumulative sum (Fig. 22 and Appendix B 3 a, Fig. 70-71) as model independent measure for the emittance growth showing a similar evolution as the emittance calculated with the Gaussian fit (Fig. 19 and Appendix B 2, Figs. 65-66). The
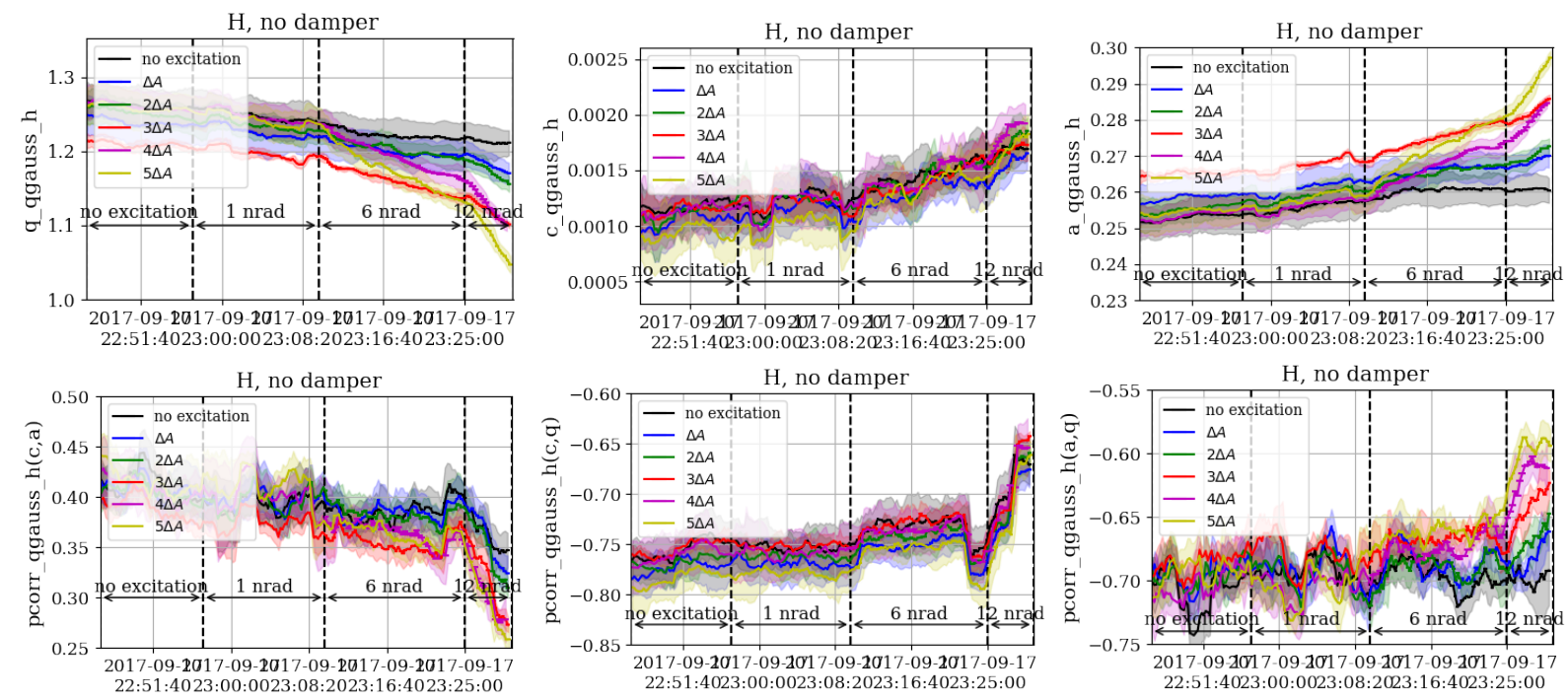

Figure 21. random excitation: fit parameter $q$ (upper left), $c$ (upper center) and $a$ (upper right), and correlation coefficient between fit parameter $c$ and $a$ (lower left), $c$ and $q$ (lower center) and $a$ and $q$ (lower right) from q-Gaussian fit of the BSRT profiles (Eqn. 9) in the horizontal plane measured with the BSRT together with the $1 \sigma$ standard deviation over the bunches with the same excitation amplitude $n \cdot \Delta A$ indicated as an envelope for each amplitude. For the fit the moving average profiles are used with no further averaging of the obtained fit parameters. 

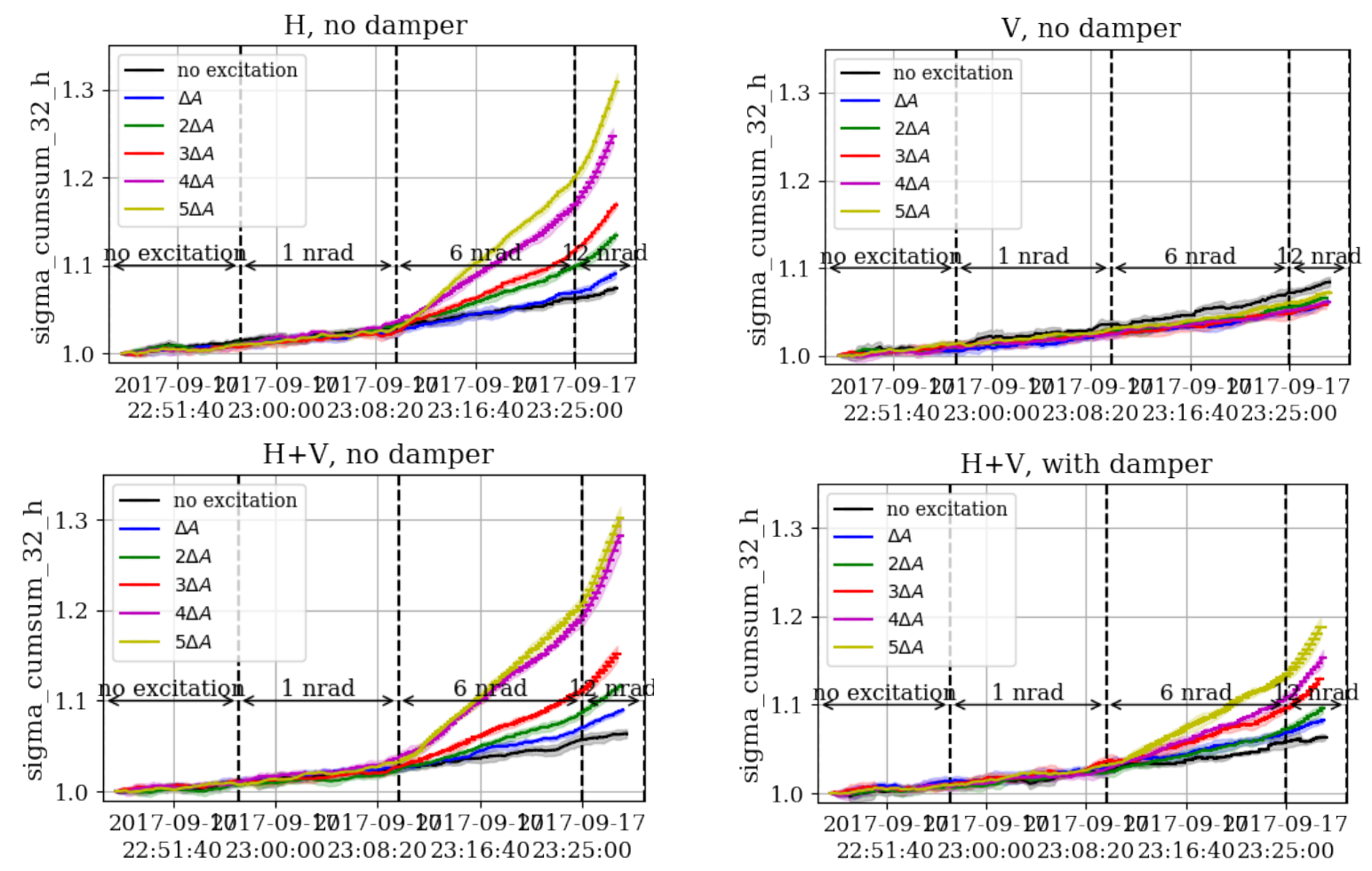

Figure 22. random uniform excitation: relative beam sigma calculated over the cumulative sum (Eqn. 17) in the horizontal plane for an excitation in $\mathrm{H}$ with transverse damper not active (upper left), $\mathrm{V}$ with transverse damper not active (upper right), $\mathrm{H}+\mathrm{V}$ with transverse damper not active (lower left) and $\mathrm{H}+\mathrm{V}$ with transverse damper active (lower right) measured with the BSRT together with the $1 \sigma$ standard deviation over the bunches with the same excitation amplitude $n \cdot \Delta A$ indicated as an envelope for each amplitude. For all beam sigma values a moving average over 10 time stamps is performed and the average, explicitly not moving average, profiles are used. The amplitudes $5 \cdot \Delta A$ and $4 \cdot \Delta A$ overlap for excitation in $\mathrm{H}+\mathrm{V}$. One reason for the behavior could be that the emittance for $4 \cdot \Delta A$ is much smaller than for $5 \cdot \Delta A$ (see Appendix B 2, Figs. 67-68)

increase of the beam sigma in the vertical plane between 22:53 and 22:55 is due to the orbit shift occurring at the same time (Fig. 16 and Appendix B 3 a, Fig. 72-73).

The change in beam sigma observed in the Gaussian fit to the BSRT profiles as well as the cumulative sum, is also very visible in the q-Gaussian fit to the profiles (Appendix B 3 b, Fig. 74, Appendix B $3 \mathrm{c}$, Fig. 83). Interpreting the distribution changes based on the q-Gaussian fit, is difficult as during the strong distribution changes encountered for $A_{\max }=12 \mathrm{nrad}$ several parameters and their correlation change:

- The fit parameter $q$ decreases with increasing excitation amplitude.

- The fit parameter $a$ increases with increasing excitation amplitude.

- The fit parameter $c$ increases with increasing excitation amplitude.

- The correlation between $c, a$ and $q$ changes. 
- The quality of the fit measured in terms of the $\chi^{2}$ of the fit stays almost constant except at the very end of the excitation for the fit in the horizontal plane.

- The qualitative behavior is the same for the bunches with transverse damper not active and transverse damper active, only that for the bunches with transverse damper active the effect is much less pronounced.

As an example the fit parameters $a, c$ and $q$ and the correlation coefficient between the same are shown in Fig. 21 for the BSRT profiles in the horizontal plane, an excitation also in the horizontal plane and the transverse damper not active. The plots for all cases are summarized in Appendix B 3 b, Figs. 77-82 for the

no damper, no excitation



V plane, slot 1698 - 2017-09-17 23:22:46, ref slot 1698 - 2017-09-17 22:57:06


no damper, with excitation $(5 \cdot \Delta A)$ in $\mathrm{H}$



V plane, slot 1532 - 2017-09-17 23:22:44, ref slot 1532 - 2017-09-17 22:57:05
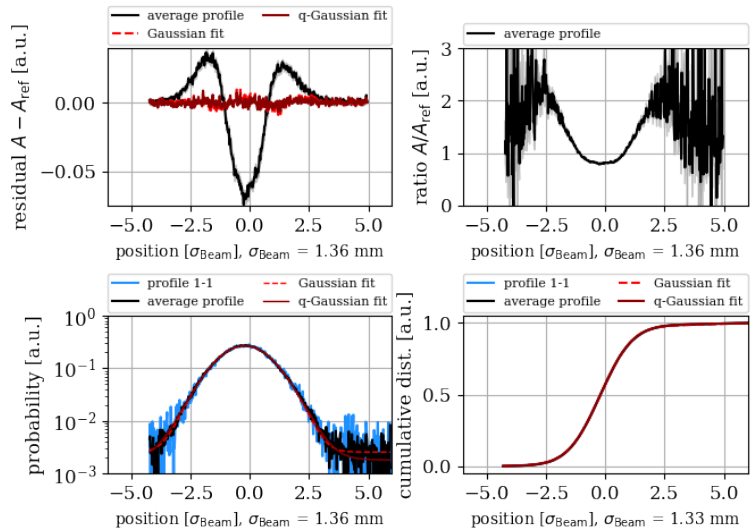

Figure 23. random uniform excitation: BSRT profile in the horizontal (top) and vertical (bottom) plane of one bunch without resonant excitation (left) and one bunch experiencing the maximum excitation (right) for an excitation only in $\mathrm{H}$ (upper right) and for an excitation only in $\mathrm{V}$ (lower right). The reference and excited bunch are taken out of the same group of 72 bunches. For all bunches the damper is not active. The residual is taken in respect to the start (2017-09-17 22:57) and end (2017-09-17 23:22) of the random excitation with a few minutes subtracted in order to account for the moving average of the profiles over 11 time stamps. Note that 22:56 is also after the closed orbit shift in the vertical plane and the different $y$-scales for the residual. The $\mathrm{x}$-axis is the position of the BSRT image projection and not the proton beam sigma. 
horizontal profiles and Appendix B 3 c, Figs. 86-91 for the vertical profiles.

The strong distribution changes are also visible on the residual and ratio of the moving average profiles suggesting:

- An increase of the beam population around $2.5-3 \sigma$

- Depletion of the center of the distribution.

Note that all profiles are normalized so that the integral over the distribution is equal to 1 . Thus an increase in one part of the distribution entails a decrease at other amplitudes. As an example the distributions of a reference bunch and a bunch experiencing the maximum excitation for an excitation only in $\mathrm{H}$ and only in $\mathrm{V}$ are compared in Fig. 23. The profiles for the other cases are summarized in Appendix B 3 d, Figs. 92-97.

\section{Comparison with simulations}

For the simulations, the same parameters as for the $7^{\text {th }}$ turn pulsing have been used (Table IV), except that a random excitation is applied instead of $7^{\text {th }}$ turn pulsing and no additional random component has been added for the distribution tracking leading to the excitation parameters listed in Table V.

Table V. Excitation applied in case of a random excitation. The other simulation parameters are the same as for the $7^{\text {th }}$ turn pulsing and are listed in IV.

\begin{tabular}{|l|c|c|}
\hline Parameter & Distribution Tracking & FMA Analysis \\
\hline $\begin{array}{l}\text { random excitaiton at } \\
\text { ADT modules (sum over 4 modules) }\end{array}$ & 0,12 and $24 \mathrm{nrad}$ & $6 \mathrm{nrad}$ \\
\hline random noise at ADT & $0 \mathrm{nrad}$ & $0 \mathrm{nrad}$ \\
\hline
\end{tabular}

The results of the distribution tracking are shown in Fig. 24. Qualitatively, the simulations agree very well with the experimental observations, explicitly:

- Emittance growth is only observed in the plane of excitation. For example, an excitation in $\mathrm{H}$ only causes emittance growth in the horizontal plane.

- The emittance growth increases with the excitation amplitude.

- In comparison to the $7^{\text {th }}$ turn pulsing, only very small losses are observed in relation to the large emittance growth.

- The bunch length stays constant, indicating that the losses are mainly transverse caused by the increasing beam size. 



Figure 24. Relative intensity (top left), bunch length (top right) and horizontal (bottom left) and vertical (bottom right) emittance obtained from simulations (Distribution Tracking) based on the 2017 injection optics with standard errors and $\left(Q_{x}, Q_{y}\right)=(62.27,60.295)$. The solid line indicates the results for no excitation, and the dotted and dashed line the results for a random excitation with 12 and $24 \mathrm{nrad}$.

Points 1 to 3 are all also experimentally observed. Point 4 is difficult to proof as in the experiment, we could not distinguish the plane in which the particles are lost. However, losses are experimentally only observed in parallel with the large transverse emittance increase encountered for $12 \mathrm{nrad}$. This indicates that the losses are also in the experiments just due to the increase of the beam sigma and thus scraping of the transverse beam tails.

The FMA analysis in frequency space for a random excitation is shown in Fig. 25 (see Appendix B 4, Fig. 98 for the FMA analysis in amplitude space). In contrast to the $n^{\text {th }}$ turn pulsing, the random excitation excites all frequencies and thus resonances. 

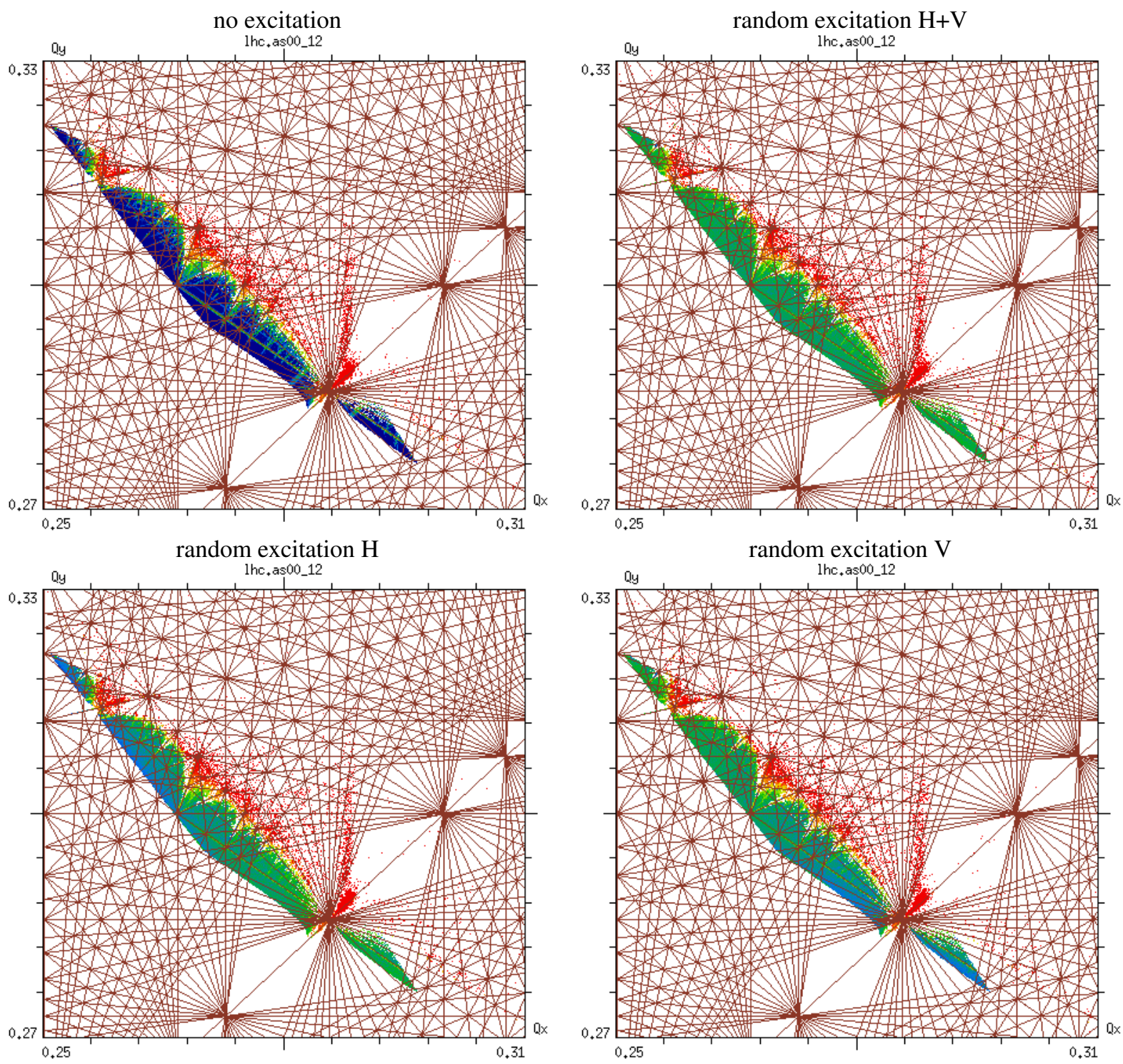

Figure 25. FMA analysis in frequency space for a random excitation based on the 2017 injection optics with no machine errors and a tune of $(62.27,60.295)$. The excitation is $1 \mathrm{nrad}$ in each/both planes. The random excitation excites all frequencies and in contrast to any $n^{\text {th }}$ turn pulsing acting only on certain resonances. 


\section{G. Fill 6220, $8^{\text {th }}$ turn}

As last excitation pattern, the $8^{\text {th }}$ turn pulsing for which only a very small effect if at all is expected from simulations (see Sec. III G 4). For this purpose, the excitation amplitude was increased up to the maximum value reachable with the ADT without risking saturation of $96 \mathrm{nrad}$.
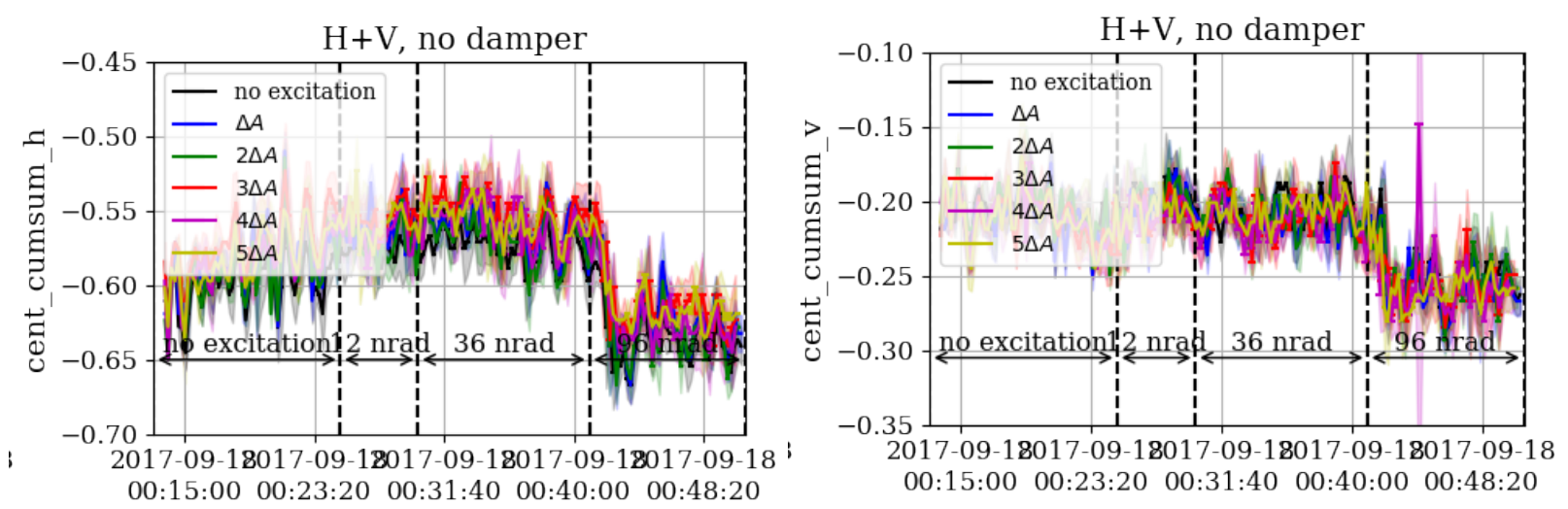

Figure 26. $8^{\text {th }}$ turn pulsing: centroid calculated over the cumulative sum (Eqn. 17) in the horizontal (left) and vertical (right) plane measured with the BSRT together with the $1 \sigma$ standard deviation over the bunches with the same excitation amplitude $n \cdot \Delta A$ indicated as an envelope for each amplitude. No moving average over the profiles nor parameter is taken in order to show the un-smoothed oscillations. The change to the maximum excitation amplitude of $A_{\max }=96 \mathrm{nrad}$ coincides with an orbit shift in the horizontal and vertical plane.

In the following sections the analysis of the FBCT data for losses (Sec. III G 1) and BSRT data for emittance (Sec. III G 2) and profiles (Sec. III G 3) is presented. The results are then compared with simulations (Sec. III G 4). Approximately isochronous with the start of the excitation with $A_{\max }=96 \mathrm{nrad}$, an orbit shift in the horizontal and vertical plane occurred (Fig. 26 and Appendix C 3 a, Figs. 107-108 for all bunches). This orbit shift did however not coincide and thus result in any change in losses, emittance or beam distribution.

\section{Losses and loss rates from FBCT measurements}

For $8^{\text {th }}$ turn pulsing, no statistically relevant increase of losses is observed for the relative bunch intensity losses as defined in Eqn. 3. As an example Fig. 27 shows the relative bunch intensity losses for an excitation in $\mathrm{H}+\mathrm{V}$. All cases are summarized in Appendix C 1, Fig. 99. A quantitative comparison of the loss rate as defined in Eqn. 4 for the three different maximum excitation amplitudes is shown in Fig. 28. For an excitation in $\mathrm{V}$ and $\mathrm{H}+\mathrm{V}$ a small increase of the loss rate is visible for $A_{\max }=96 \mathrm{nrad}$. In summary, losses are very small and it is questionable if the small increase visible in Fig. 28 is statistically relevant. 

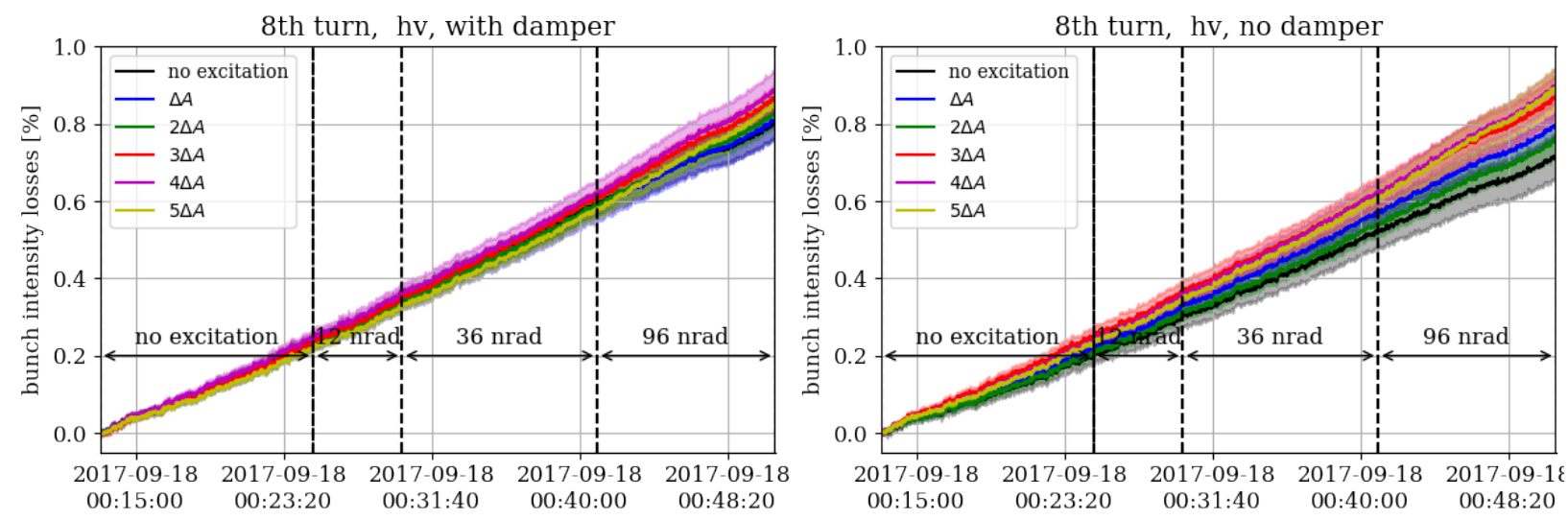

Figure 27. $8^{\text {th }}$ turn pulsing: Relative bunch intensity losses measured with the FBCTs (Eqn. 3). The average over the group of 6 bunches experiencing the same excitation amplitude is indicated by a solid line together with the $1 \sigma$ standard deviation over the four bunches indicated as an envelope in the same color. The maximum excitation amplitudes are indicated with black arrows. There is no dependence of the losses on the excitation amplitude.
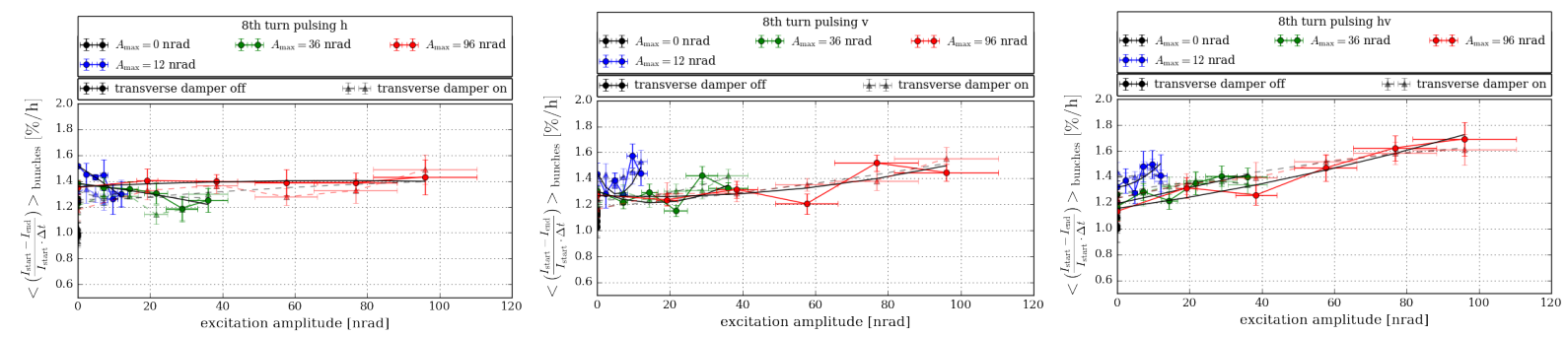

Figure 28. $8^{\text {th }}$ turn pulsing: Average bunch intensity loss rate measured with the FBCTs (Eqn. 4) for an excitation only in $\mathrm{H}$ (left), only in $\mathrm{V}$ (center) and in $\mathrm{H}+\mathrm{V}$ (right). The bunches for which the transverse damper is active are shown with solid dots and the bunches for which the transverse damper is not active are shown with slightly transparent triangles. Each point represents the average over the six bunches with the same excitation amplitude $n \cdot \Delta A$. The second order polynomial fit to the loss rate is indicated with a solid line for the bunches with transverse damper active and with a dashed line for the bunches with the transverse damper not active. The error on the excitation amplitude during this MD is approximately $10-15 \%$, where the upper limit of $15 \%$ has been assumed for this plot.

\section{BSRT emittance from LHC logging database (Gaussian fit)}

For $8^{\text {th }}$ turn pulsing, a small emittance increase is observed in the horizontal plane for an excitation in $\mathrm{H}$ and in $\mathrm{H}+\mathrm{V}$ and no emittance growth in the vertical plane. However, it is difficult to disentangle the dependence of the emittance growth due to the excitation and the difference in emittance growth due to the different initial emittances. Meaning, a smaller initial emittance usually results in a larger relative emittance growth. As shown in Fig. 29, the excitation amplitude dependent emittance growth is for example not visible for pulsing in $\mathrm{H}+\mathrm{V}$ due to the smaller initial emittances compensating the effect of the excitation. A strong dependence is on the other hand observed for pulsing only in $\mathrm{H}$, in which case the emittance growth due to the excitation adds up with the emittance growth due to the smaller initial emittance. The emittance and relative emittances for the other cases are summarized in Appendix C2, Figs. 100-103 and the scaling of 
the emittance with the excitation amplitude in Appendix C2, Fig. 104.
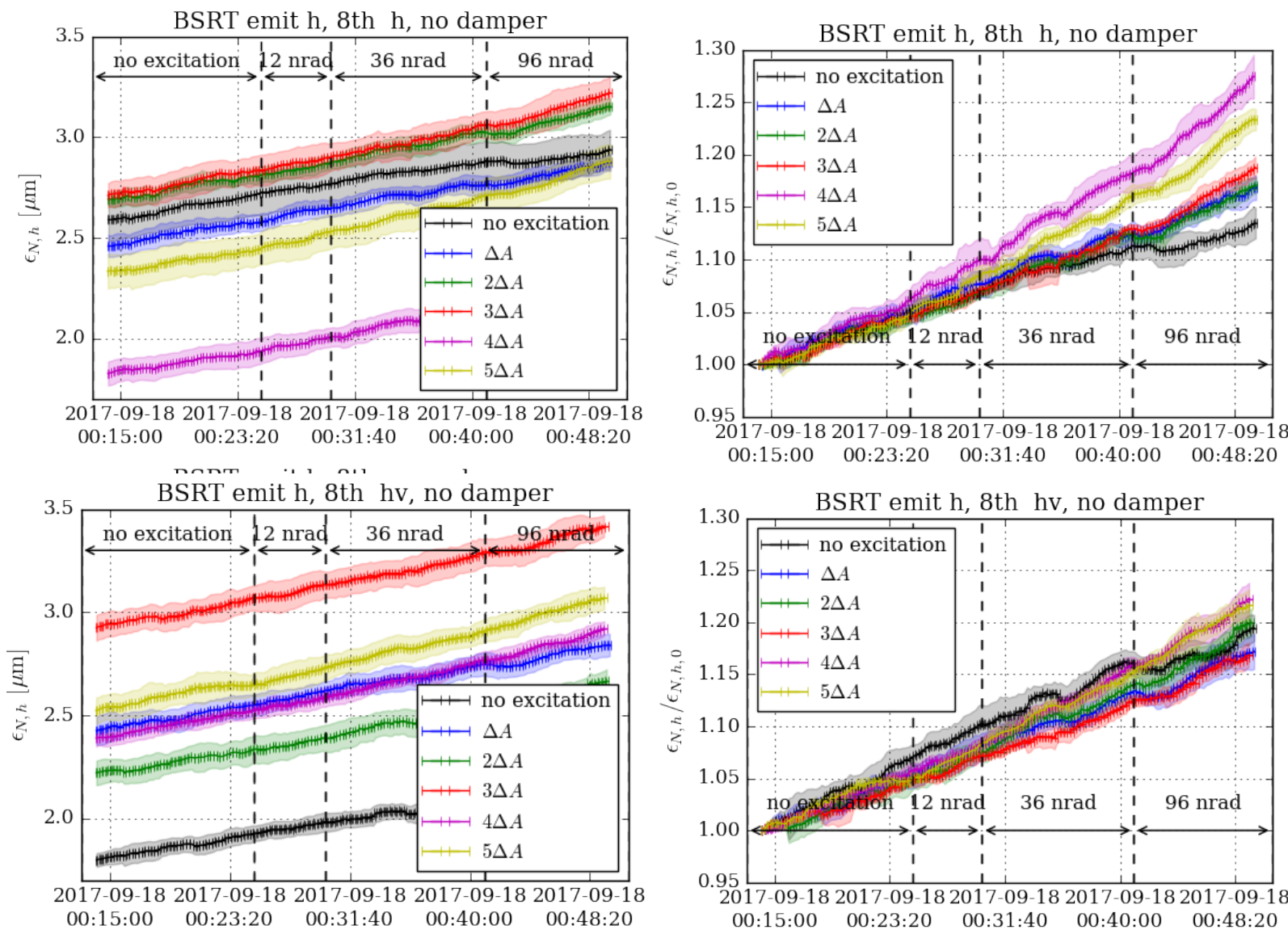

Figure 29. $8^{\text {th }}$ turn pulsing: emittance (left) and relative emittance (right) in the horizontal plane measured with the BSRT (Gaussian fit) and as defined in Eqn. 24 together with the $1 \sigma$ standard deviation over the bunches with the same excitation amplitude $n \cdot \Delta A$ indicated as an envelope for each amplitude. For all emittance values a moving average over 10 time stamps is performed.

\section{BSRT profiles}

Same as for the $7^{\text {th }}$ turn pulsing and random excitation, we can use the beam sigma calculated over the cumulative sum (Fig. 30 and Appendix C 3 a, Fig. 105-106) as model independent measure for the emittance growth showing a similar evolution as the emittance calculated with the Gaussian fit (Fig. 29 and Appendix C2, Figs. 100-101).

The change in beam sigma in the horizontal plane observed in the Gaussian fit to the BSRT profiles as well as the cumulative sum is also visible in the parameters of the q-Gaussian fit to the profiles:

- The beam sigma increases with increasing excitation amplitude.

- The fit parameter $q$ decreases with increasing excitation amplitude. 

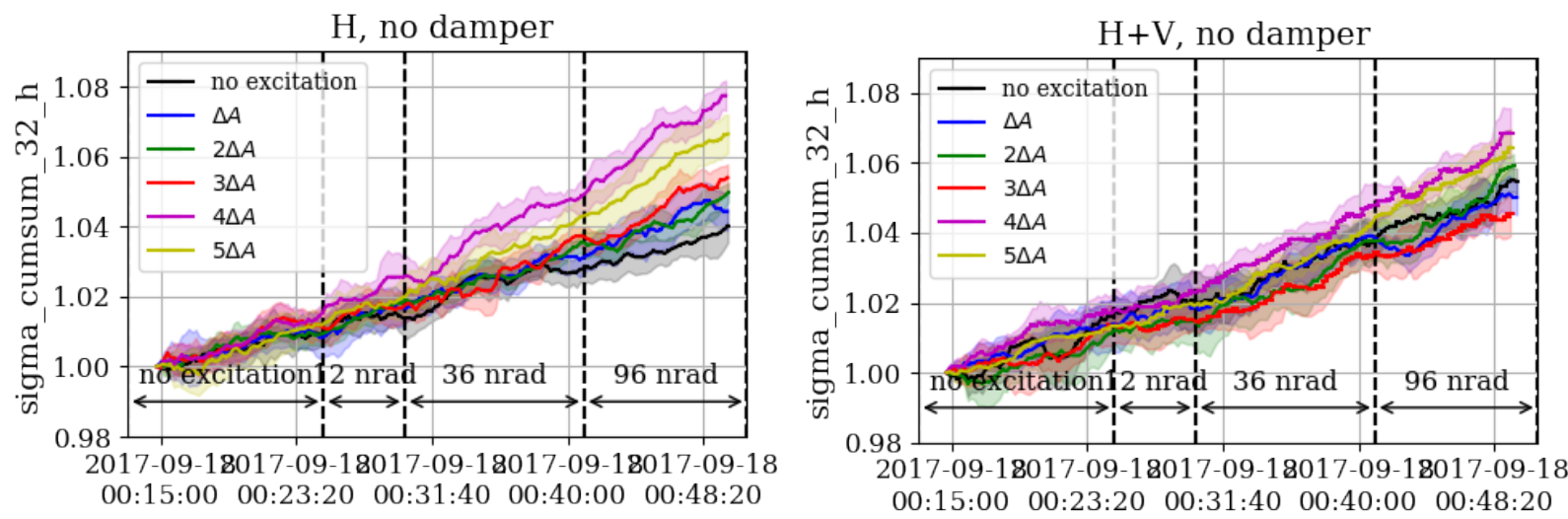

Figure 30. $8^{\text {th }}$ turn pulsing: relative beam sigma calculated over the cumulative sum (Eqn. 17) in the horizontal plane for an excitation in $\mathrm{H}$ (left) and $\mathrm{H}+\mathrm{V}$ (right) with the transverse damper not active and measured with the BSRT together with the $1 \sigma$ standard deviation over the bunches with the same excitation amplitude $n \cdot \Delta A$ indicated as an envelope for each amplitude. For all beam sigma values a moving average over 10 time stamps is performed and the average, explicitly not moving average, profiles are used.
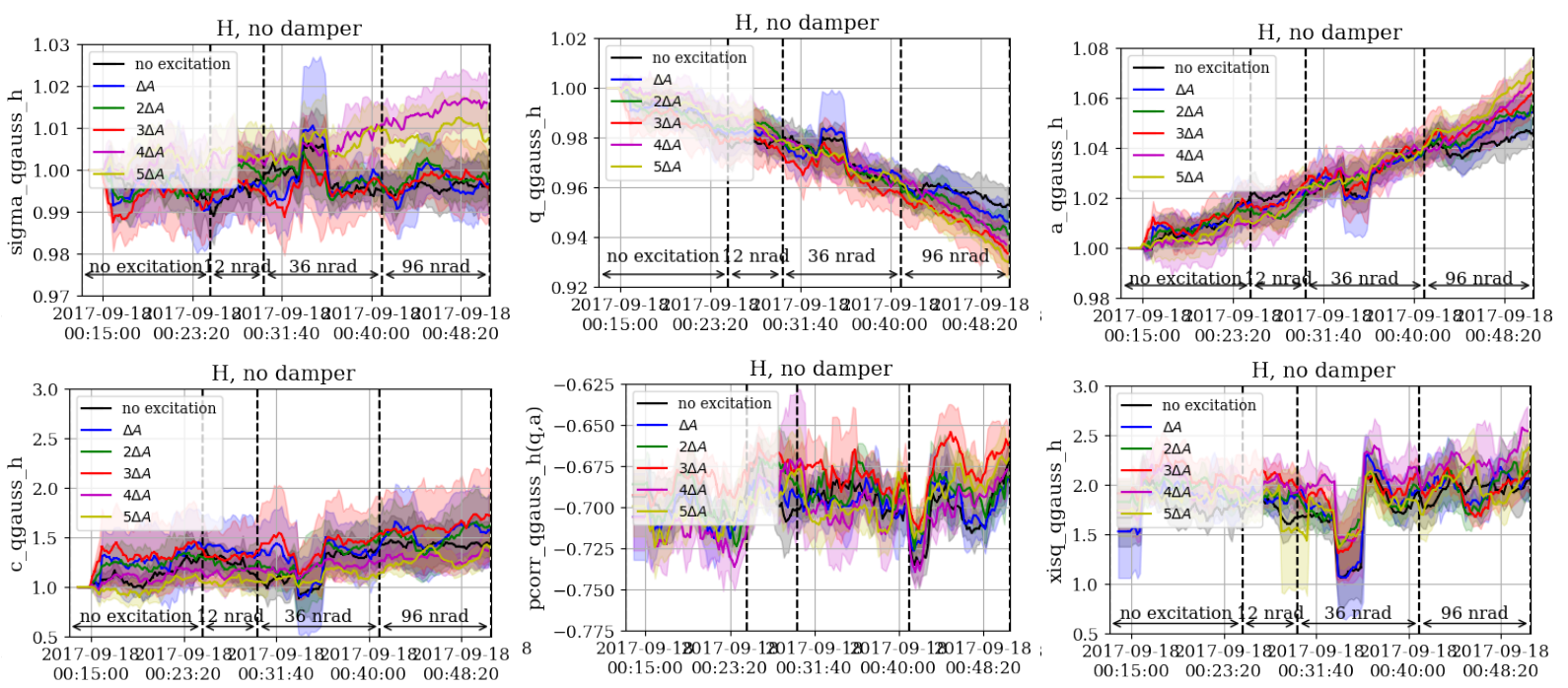

Figure 31. $8^{\text {th }}$ turn pulsing: relative beam sigma (upper left) and relative fit parameters $q$ (upper center), $a$ (upper right), $c$ (lower left), as well as correlation coefficient between fit parameter $a$ and $q$ (lower center) and $\chi^{2}$ (lower right) from q-Gaussian fit of the BSRT profiles (Eqn. 9) in the horizontal plane measured with the BSRT together with the $1 \sigma$ standard deviation over the bunches with the same excitation amplitude $n \cdot \Delta A$ indicated as an envelope for each amplitude. For the fit the moving average profiles are used with no further averaging of the obtained fit parameters.

- The fit parameter $a$ increases with increasing excitation amplitude.

- The correlation between $a$ and $q$ stays constant and does not depend on the excitation amplitude.

- The evolution of the fit parameter $c$ does not depend on the excitation amplitude.

- The quality of the fit measured in terms of the $\chi^{2}$ of the fit stays constant. 
- The behavior is the same for the bunches with transverse damper not active and transverse damper active.

As an example Fig. 31 shows the parameters of interest for the BSRT profiles in the horizontal plane, an excitation also in the horizontal plane and the transverse damper not active. The plots for all cases are summarized in Appendix C 3 b, Figs. 109-117 for the horizontal profiles. In the vertical plane, no changes are observed (Appendix B 3 c, Figs. 118-126).
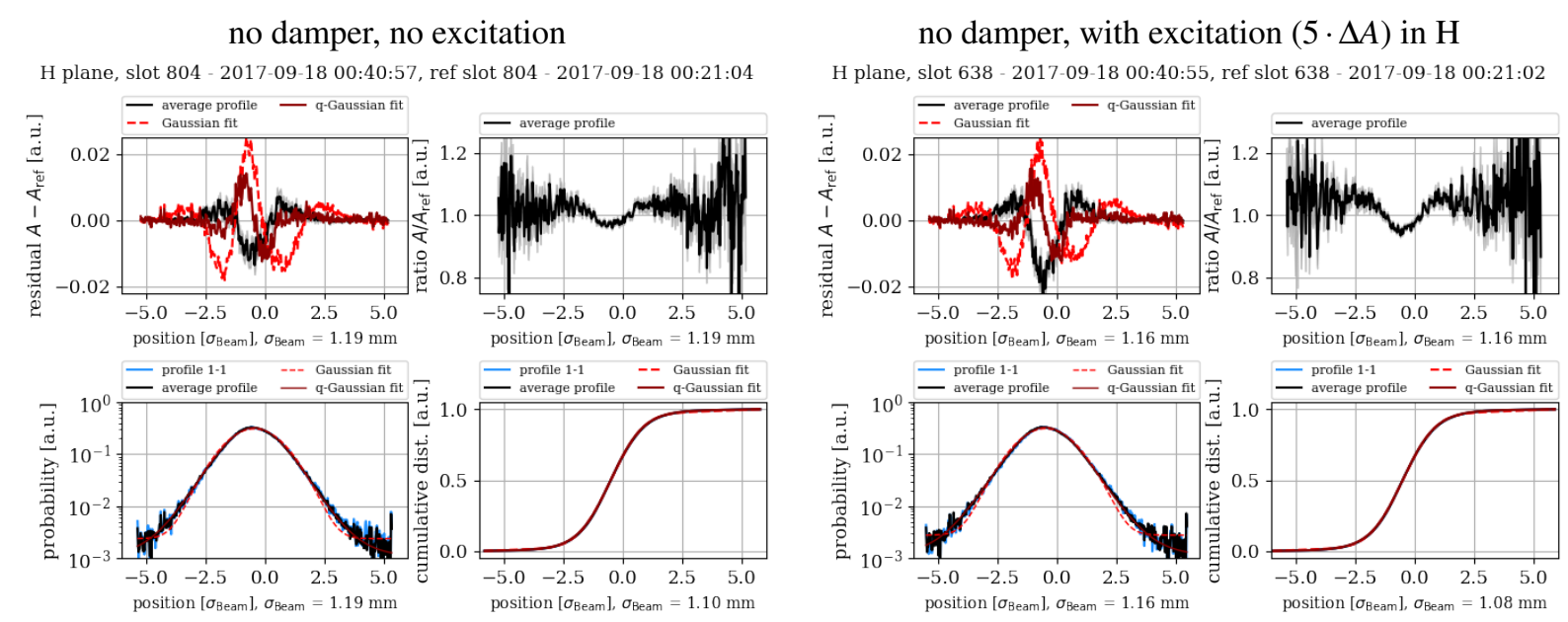

Figure 32. $8^{\text {th }}$ turn pulsing: BSRT profile in the horizontal plane of one bunch without resonant excitation (left) and one bunch experiencing the maximum excitation (right) for an excitation only in $\mathrm{H}$. The reference and excited bunch are taken out of the same group of 72 bunches. For all bunches the damper is not active. The residual is taken in respect to the start (2017-09-18 00:21) and end (2017-09-18 00:40) of the $8^{\text {th }}$ turn pulsing with a few minutes subtracted in order to account for the moving average of the profiles over 11 time stamps. The x-axis is the position of the BSRT image projection and not the proton beam sigma.

The slight changes of the distribution described above are even visible in the profiles itself. As an example Fig. 32 compares the profiles in the horizontal plane of a reference bunch and a bunch experiencing the maximum excitation $5 \cdot \Delta A$ in $\mathrm{H}$. Both bunches also have approximately the same emittance, explicitly $\varepsilon_{N, h}($ slot 804$)=3.0578$ and $\varepsilon_{N, h}($ slot 638$)=3.0134$, the change in distribution can thus be attributed to the excitation and not any excitation independent difference in emittance growth due to different initial emittances.

The profiles of one representative bunch per case are summarized in Appendix C 3 d, Figs. 127-132. Note that for example for an excitation in $\mathrm{H}+\mathrm{V}$ with damper not active the distribution of the reference and excited bunch look equivalent, however the initial emittance of the reference bunch is much smaller than the one of the excited bunch and therefore both bunches experience an equivalent emittance growth however from different sources (Appendix C 3 d, Fig. 129).

In summary, the changes in the fit parameter suggest the following distribution changes:

- A change in distribution in the horizontal plane. As both fit parameters $a$ and $q$ are strongly correlated it is however difficult to translate this change in a change of population for certain amplitudes. 
- The transverse damper has no effect.

- The vertical distribution does not change.

The changes in the residual and ratio of the profile suggest consistent with the change in the $q$-Gaussian fit parameters:

- A depletion of the beam core in the horizontal plane.

- An increase of the distribution around $2.0 \sigma$ in the horizontal plane.

- No effect of the transverse damper.

- No changes of the vertical distribution.

\section{Comparison with simulations}

For the simulations, the same parameters as for the $7^{\text {th }}$ turn pulsing have been used (Table IV), except that $8^{\text {th }}$ turn pulsing with higher excitation amplitudes is applied instead of $7^{\text {th }}$ turn pulsing leading to the excitation parameters listed in Table VI. Which resonances are excited by the $8^{\text {th }}$ turn pulsing and

Table VI. Excitation applied in case of a random excitation. The other simulation parameters are the same as for the $7^{\text {th }}$ turn pulsing and are listed in IV.

\begin{tabular}{|l|c|c|}
\hline Parameter & Distribution Tracking & FMA Analysis \\
\hline $8^{\text {th }}$ turn pulsing at & 0,96 and $192 \mathrm{nrad}$ & $96 \mathrm{nrad}$ \\
ADT modules (sum over 4 modules) & & \\
\hline random noise at ADT & $6 \mathrm{nrad}$ & - \\
\hline
\end{tabular}

their amplitude dependence is best seen from the FMA plot in amplitude space shown in Fig. 33 (see Appendix C4, Fig. 133 for the FMA analysis in frequency space). For a horizontal excitation the $16 Q_{y}$ resonance is excited, for a vertical excitation the $8 Q_{x}-4 Q_{y}$ and for an excitation in both planes obviously both resonances. These results are a bit puzzling as a horizontal excitation should not drive a vertical resonance, here $16 Q_{y}$.

The results of the distribution tracking are shown in Fig. 34. In summary the following observations can be made:

- No increase in losses.

- No change in bunch length.

- Small emittance growth in the horizontal and vertical plane with no clear dependence on the excitation plane. 

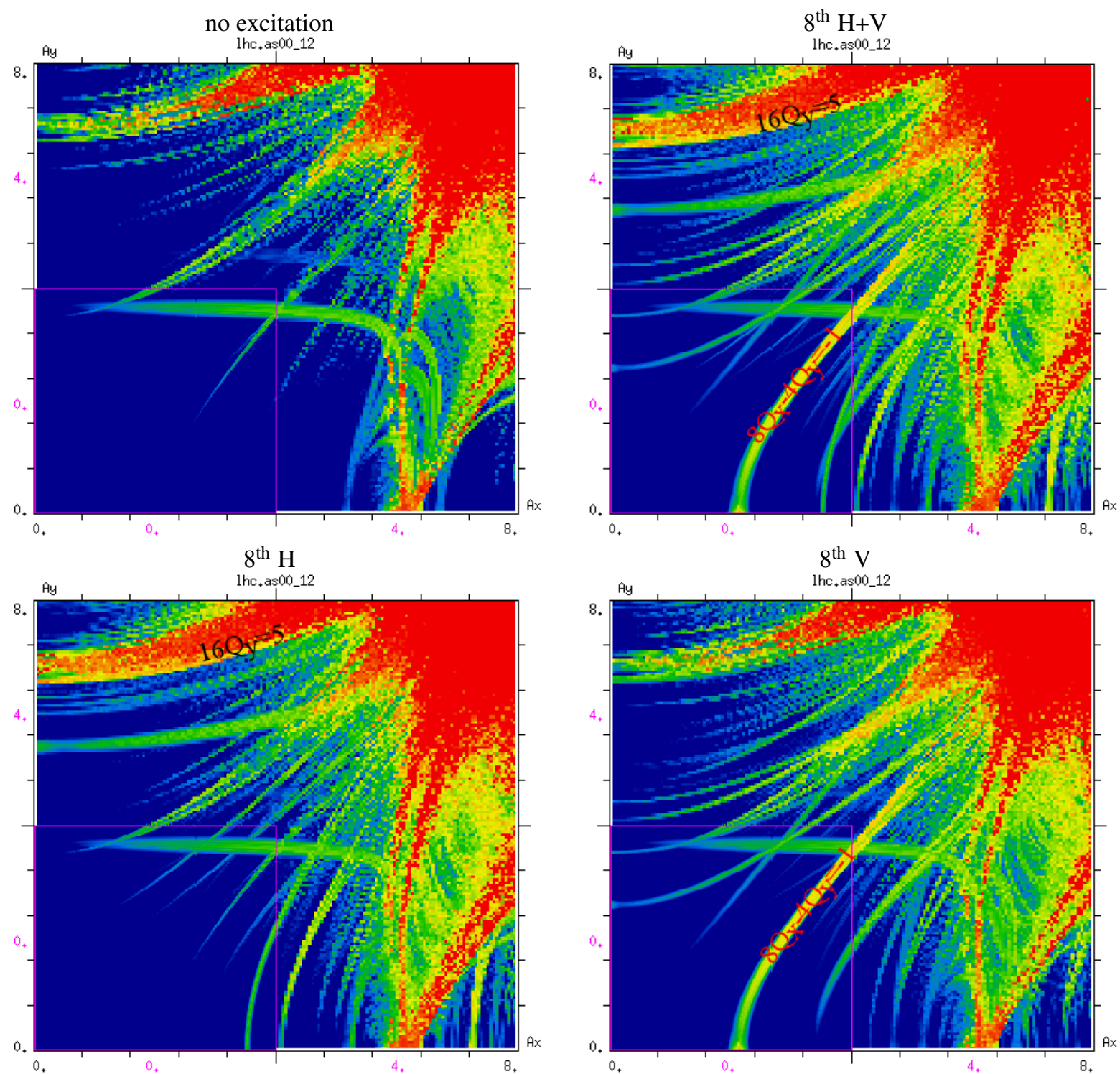

Figure 33. FMA analysis in amplitude space for $8^{\text {th }}$ turn pulsing based on the 2017 injection optics with no machine errors and a tune of $(62.27,60.295)$. The excitation is $96 \mathrm{nrad}$ in each/both planes. For a horizontal excitation the $16 Q_{y}$ resonance is excited, for a vertical excitation the $8 Q_{x}-4 Q_{y}$ and for an excitation in both planes obviously both resonances. The excitation of the $16 Q_{y}$ resonance for a horizontal excitation is surprising and might be just an artifact of the simulation.

The effect of the $8^{\text {th }}$ turn pulsing in simulations is in general very small which is in accordance with the experimental results. In experiments, only the horizontal emittance changes for an excitation in $\mathrm{H}$ and $\mathrm{H}+\mathrm{V}$. In simulations, this sensitivity to the excitation plane could however not be reproduced. 



Figure 34. Relative intensity (top left), bunch length (top right) and horizontal (bottom left) and vertical (bottom right) emittance obtained from simulations (Distribution Tracking) based on the 2017 injection optics with standard errors and $\left(Q_{x}, Q_{y}\right)=(62.27,60.295)$. The solid line indicates the results for no excitation, and the dotted and dashed line the results for $8^{\text {th }}$ turn pulsing with 96 and 192 nrad.

\section{H. Influence of the transverse damper}

All measurements during this MD were always conducted with the transverse damper active on half of the bunches (with damper) and not active on the other half of the bunches (no damper). Comparing the results for both cases, the damper does not reduce the loss rate or emittance nor change the distribution in case of any $n^{\text {th }}$ turn pulsing pattern tried during the MD in 2016 [1] and this MD (see Sec. III E and Sec. III G). One explanation is that the excitation lies outside of the bandwidth of the transverse damper which is narrowly set around the transverse tunes. For a random excitation, the damper considerably decreases the loss rate, emittance growth and also distribution changes (see Sec. III F). 


\section{CONCLUSIONS}

The ultimate aim of this MD in 2017 and the first MD in 2016 [1] was to define tolerances on the kick of the e-lens on the particles in the beam core for HL-LHC in case of a pulsed operation. In the MD in 2016 the 10th turn and $7^{\text {th }}$ turn pulsing were successfully tested. The aim of this MD in 2017 was now to:

- Confirm the results of the $7^{\text {th }}$ turn pulsing.

- Test one $n^{\text {th }}$ turn pulsing pattern for which a very small/no effect on the beam is expected. Here the $8^{\text {th }}$ turn pulsing was tried.

- Test the random excitation in comparison to the $n^{\text {th }}$ turn pulsing.

In summary the following results were obtained for the above pulsing patterns:

$7^{\text {th }}$ turn pulsing:: For an excitation in $\mathrm{H}, \mathrm{V}$ and $\mathrm{H}+\mathrm{V}$ with an excitation amplitude of 6 nrad and $12 \mathrm{nrad}$, excitation amplitude dependent losses were observed and the loss rates were comparable for all three cases. In the horizontal plane, the emittance growth is independent of the excitation. In the vertical plane, an excitation amplitude dependent emittance growth is observed for pulsing in $\mathrm{V}$ and $\mathrm{H}+\mathrm{V}$. The BSRT profile analysis reveals an increase of the vertical beam distribution around $2.5-3 \sigma$ and a depletion of the beam core. In the horizontal plane, the BSRT profiles are not changed by the excitation. Loss rates were in general comparable to the 2016 MD results. The absence of emittance growth for an excitation in $\mathrm{H}$ furthermore agreed with the results of 2016 featuring also no emittance growth. Note that the optics and working point differ for 2016 and 2017. This entailed that not only the $7 Q_{x}$ resonance was excited as in 2016 , but in addition also the $7 Q_{y}$ and $7 Q_{x}+7 Q_{y}$.

$8^{\text {th }}$ turn pulsing:: Here the excitation amplitude was raised to the maximum kick amplitude achievable with the ADT without risking any saturation effects, explicitly $12 \mathrm{nrad}, 36 \mathrm{nrad}$ and finally the maximum of $96 \mathrm{nrad}$ were tried. For an excitation in V and $\mathrm{H}+\mathrm{V}$ very small excitation amplitude dependent losses were observed. In the horizontal plane, a small excitation amplitude dependent increase is observed for pulsing in $\mathrm{H}$ and $\mathrm{H}+\mathrm{V}$. In the vertical plane, the emittance growth is independent of the excitation. These small changes in the vertical plane could also be detected with the BSRT profiles, which reveal an increase of the horizontal beam distribution around $2.0 \sigma$ and a depletion of the beam core. In the vertical plane, the profiles are not changed by the excitation.

random uniform excitation:: From a random excitation, the strongest effect is expected and therefore only very small excitation amplitudes were tried, explicitly $1 \mathrm{nrad}, 6$ nrad and $12 \mathrm{nrad}$. For $1 \mathrm{nrad}$, no effect on the beam was observed. For 6 nrad and 12 nrad excitation amplitude dependent losses were detected which can be attributed to the large emittance growth. Large emittance growth occurred in the plane of excitation. For example a horizontal excitation resulted only in horizontal emittance 
growth and no changes in the vertical plane. These changes could also be directly seen in the BSRT profiles showing an increase of the distribution around $2.5-3 \sigma$ and a depletion of the beam core.

In agreement with the simulations, the experiments furthermore revealed the following differences between a resonant excitation (here 7th, 8th and 10th turn pulsing) and a random excitation:

- A resonant excitation acts on certain resonances while a random excitation excites all frequencies. This implies, that the effect of the different $n^{\text {th }}$ turn pulsing patterns can differ widely depending on $n$.

- A random excitation mainly results in emittance growth in the plane of excitation. With each increase of the excitation amplitude, the emittance growth adjusts immediately to a new constant growth rate. Losses occur and increase rapidly once the aperture starts eating into the transverse beam distribution.

- A resonant excitation does not necessarily result in emittance growth, but might also cause primarily losses indicating a higher diffusion in the beam tails without affecting the core. If emittance growth is observed, the emittance first undergoes a fast strong increase and then adjusts to new constant growth rate. A further increase of the maximum excitation amplitude does not change this new growth rate any more. This behavior is explained by a fast adjust of the beam distribution to a new equilibrium state, which is also observed in simuations.

\section{FURTHER ANALYSIS AND OUTLOOK}

In this note the data acquired with the diamond BLMs has not been analyzed. This data could give more insight into losses and also confirm the measurements with the FBCTs.

As could be shown in simulations, the effect of the resonant excitation changes if random noise is included. As at injection the natural noise of the LHC is a mixture of many different noise sources, e.g. ground motion, magnetic field ripple, and is not well known, it is in general challenging to compare simulations and experiments. At top energy, the natural noise of the LHC is better understood and we therefore recommend to repeat the experiment at top energy.

\section{ACKNOWLEDGEMENTS}

We wish to thank the whole OP team for a succesful MD, Hannes Bartosik, Heiko Damerau and Verena Kain for their help with the injection scheme, Riccardo De Maria for his help with the preparation of the MAD-X input files and Enrico Bravin and Georges Trad for their help with the BSRT profile analysis. 
[1] Miriam Fitterer, Giulio Stancari, Alexander Valishev, Roderik Bruce, Parthena Stefania Papadopoulou, Giulia Papotti, Dario Pellegrini, Stefano Redaelli, Georges Trad, Daniel Valuch, Gianluca Valentino, Joschka Wagner, and Chen Xu. Effect of a resonant excitation on the evolution of the beam emittance and halo population. Technical Report CERN-ACC-NOTE-2017-0037, May 2017.

[2] Review on the needs for a hollow e-lens for the HL-LHC, October 2016.

[3] G. Stancari, A. Valishev, G. Annala, G. Kuznetsov, V. Shiltsev, D. A. Still, and L. G. Vorobiev. Collimation with hollow electron beams. Phys. Rev. Lett., 107:084802, Aug 2011.

[4] Valentina Previtali, Giulio Stancari, Alexander Valishev, and Stefano Redaelli. Simulation study of hollow electron beam collimation for LHC. Technical Report FERMILAB-TM-2584-APC, Fermilab, May 2014.

[5] Valentina Previtali, Giulio Stancari, Alexander Valishev, and Stefano Redaelli. Numerical simulations of a proposed hollow electron beam collimator for the LHC upgrade at CERN. Technical Report FERMILAB-TM2560-APC, Fermilab, July 2013.

[6] Miriam Fitterer, Giulio Stancari, and Alexander Valishev. Simulation study of hollow electron beam collimation in HL-LHC. Technical Report FERMILAB-TM-2636-AD, Fermilab, Oct. 2016.

[7] Xiao-Long Zhang, Kip Bishofberger, Vsevolod Kamerdzhiev, Valery Lebedev, Vladimir Shiltsev, Randy Thurman-Keup, and Alvin Tollestrup. Generation and diagnostics of uncaptured beam in the Fermilab Tevatron and its control by electron lenses. Phys. Rev. ST Accel. Beams, 11:051002, May 2008.

[8] Miriam Fitterer, Giulio Stancari, and Alexander Valishev. Effect of pulsed hollow electron-lens operation on the proton beam core in LHC. Technical Report FERMILAB-TM-2635-AD, Fermilab, Oct. 2016.

[9] private communication H. Bartosik.

[10] Miriam Fitterer, Fanouria Antoniou, Giulio Stancari, Papadopoulou Stefania, and Alexander Valishev. Analysis of BSRT profiles in the LHC at injection. Technical Report to be published, Fermilab, Feb. 2017.

[11] private communication R. Tomás.

[12] Dmitry Shatilov, Yuri Alexahin, Valeri Lebedev, and Alexander Valishev. Lifetrac code for the weak-strong simulation of the beam-beam effects in Tevatron. Conf. Proc., (FERMILAB-CONF-05-246-AD, PAC-2005TPAT084), 2005.

[13] Miriam Fitterer, Giulio Stancari, and Alexander Valishev. Effect of pulsed hollow electron-lens operation on the proton beam core in LHC. Technical Report FERMILAB-TM-2635-AD, Fermilab, Oct. 2016.

[14] Xavier Buffat, Nicolo Biancacci, Sondre Vik Furuseth, Delphine Jacquet, Elias Metral, Dario Pellegrini, Mirko Pojer, Georges Trad, Daniel Valuch, Javier Barranco Garcia, Tatiana Pieloni, Claudia Tambasco, and Qiang Li. Probing the behaviour of high brightness bunches in collision at $6.5 \mathrm{TeV}$ and the interplay with an external source of noise (MD1433). Apr 2017.

[15] Javier Barranco Garcia, Xavier Buffat, Tatiana Pieloni, Claudia Tambasco, Georges Trad, Daniel Valuch, Michael Betz, Manfred Wendt, Mirko Pojer, Matteo Solfaroli Camillocci, Belen Maria Salvachua Ferrando, Kajetan Fuchsberger, Markus Albert, and Ji Qiang. Md 400: LHC emittance growth in presence of an external source of noise during collision. Technical Report CERN-ACC-NOTE-2016-0020, CERN, Jan. 2016. 


\section{Appendix A: Additional figures for fill 6220, $7^{\text {th }}$ turn pusling}

\section{1. $7^{\text {th }}$ turn pulsing: beam losses measured with FBCT}
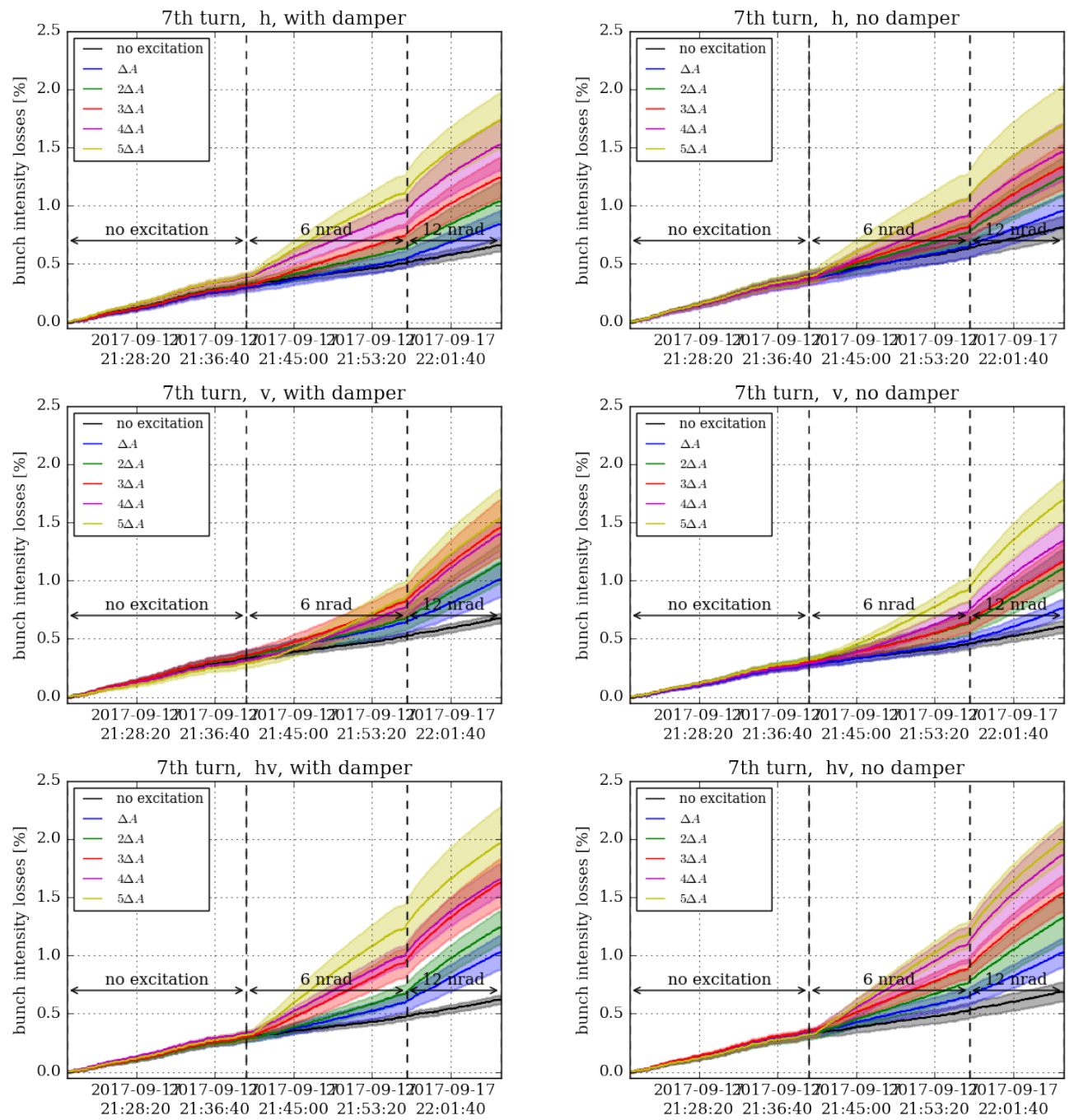

Figure 35. $7^{\text {th }}$ turn pulsing: Relative bunch intensity losses measured with the FBCTs (Eqn. 3). The average over the group of 6 bunches experiencing the same excitation amplitude is indicated by a solid line together with the $1 \sigma$ standard deviation over the four bunches indicated as an envelope in the same color. The maximum excitation amplitudes are indicated with black arrows. A clear increase of the losses with the excitation amplitude within the bunch train $(n \cdot \Delta A)$ and at each increase of the maximum amplitude $\left(A_{\max }\right)$ is visible. The losses caused by an excitation in the horizontal plane are sightly larger than the ones in the vertical plane. The damper does not appear to have any effect. 


\section{2. $7^{\text {th }}$ turn pulsing: BSRT emittance as logged in the LHC Logging database}


Figure 36. $7^{\text {th }}$ turn pulsing: relative emittance in the horizontal plane measured with the BSRT (Gaussian fit) and as defined in Eqn. 24 together with the $1 \sigma$ standard deviation over the bunches with the same excitation amplitude $n \cdot \Delta A$ indicated as an envelope for each amplitude. For all emittance values a moving average over 10 time stamps is performed. 

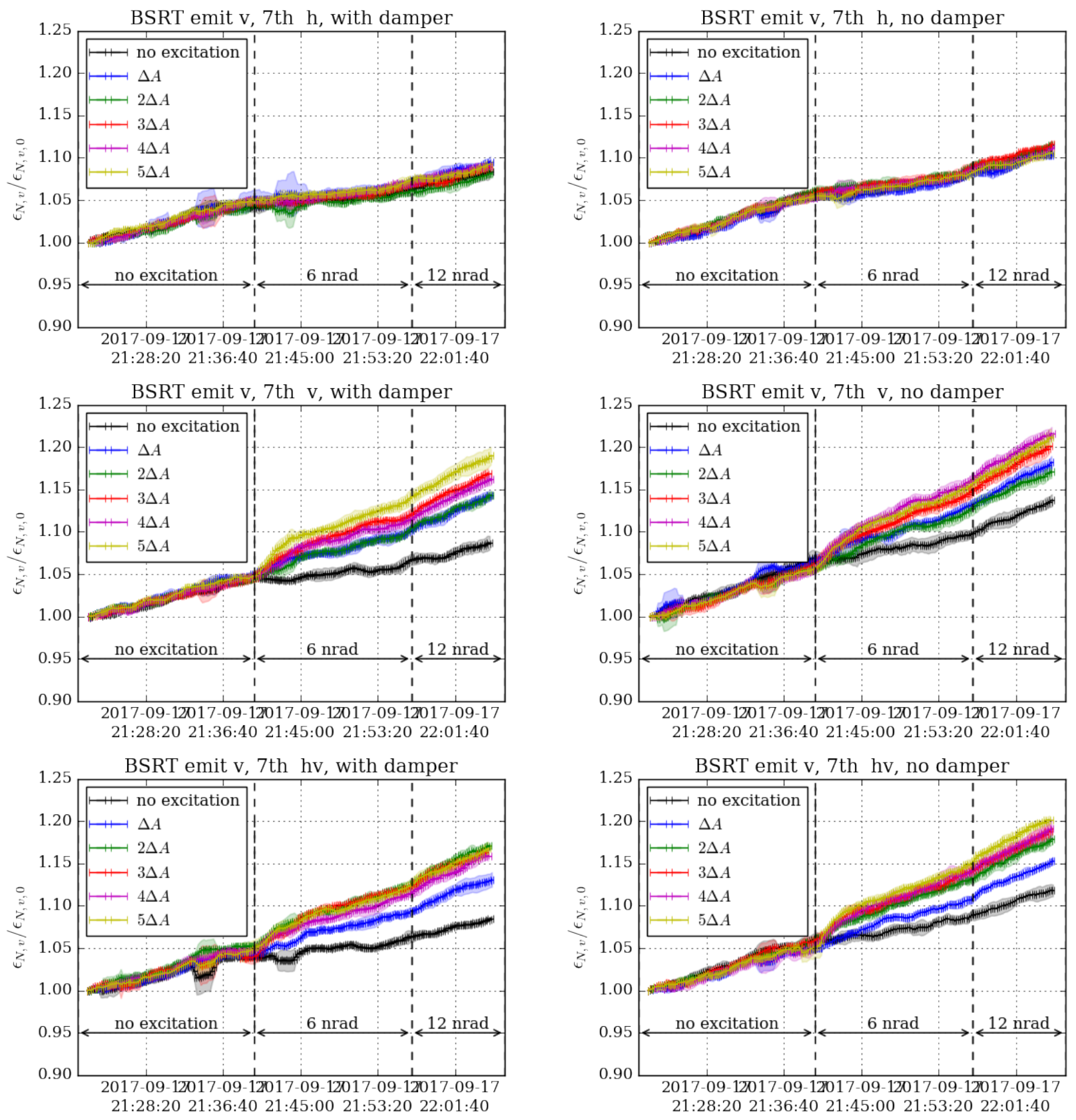

Figure 37. $7^{\text {th }}$ turn pulsing: relative emittance in the vertical plane measured with the BSRT (Gaussian fit) and as defined in Eqn. 24 together with the $1 \sigma$ standard deviation over the bunches with the same excitation amplitude $n \cdot \Delta A$ indicated as an envelope for each amplitude. For all emittance values a moving average over 10 time stamps is performed. A clear excitation amplitude dependent emittance growth for pulsing in $\mathrm{V}$ and $\mathrm{H}+\mathrm{V}$ is visible. 


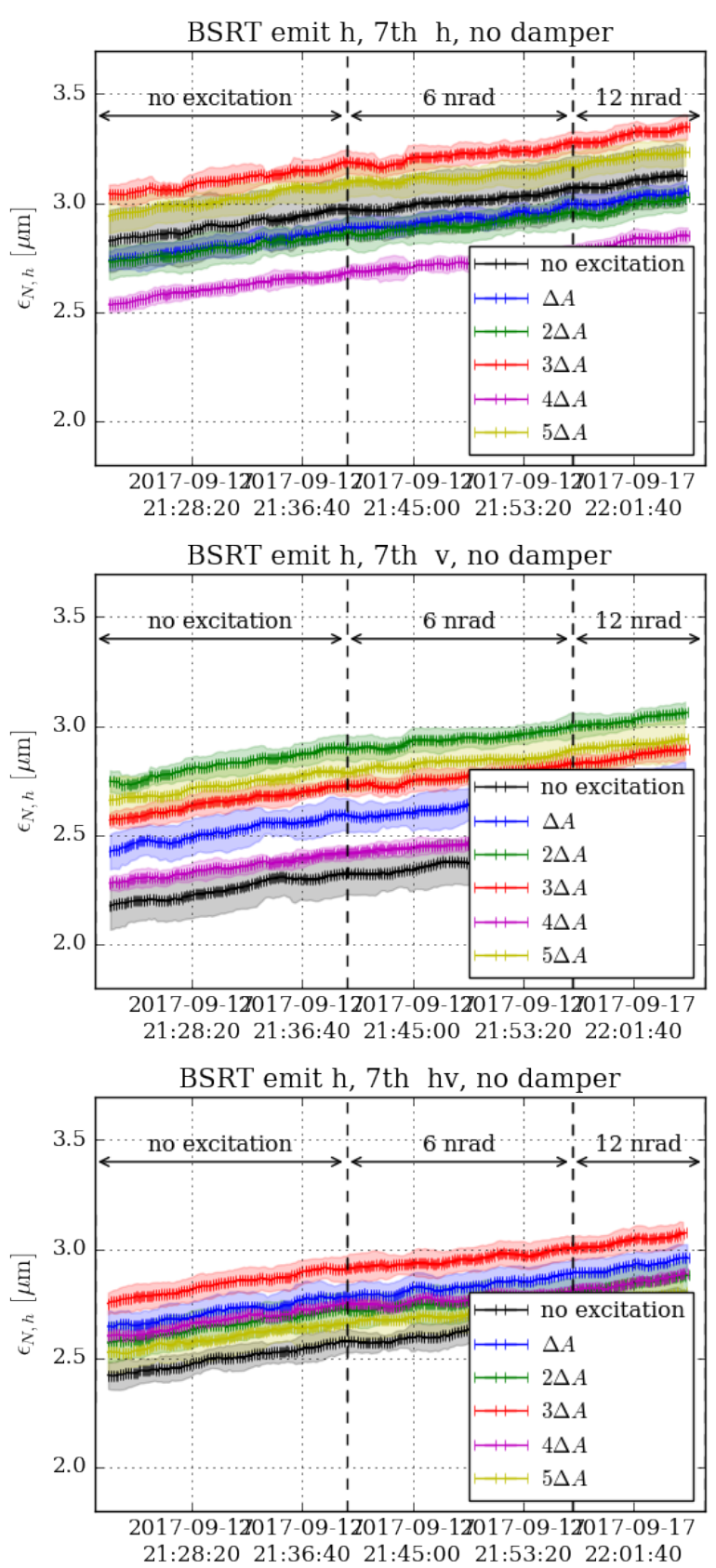

Figure 38. $7^{\text {th }}$ turn pulsing: emittance in the horizontal plane measured with the BSRT (Gaussian fit) and as defined in Eqn. 22 together with the $1 \sigma$ standard deviation over the bunches with the same excitation amplitude $n \cdot \Delta A$ indicated as an envelope for each amplitude. For all emittance values a moving average over 10 time stamps is performed. 

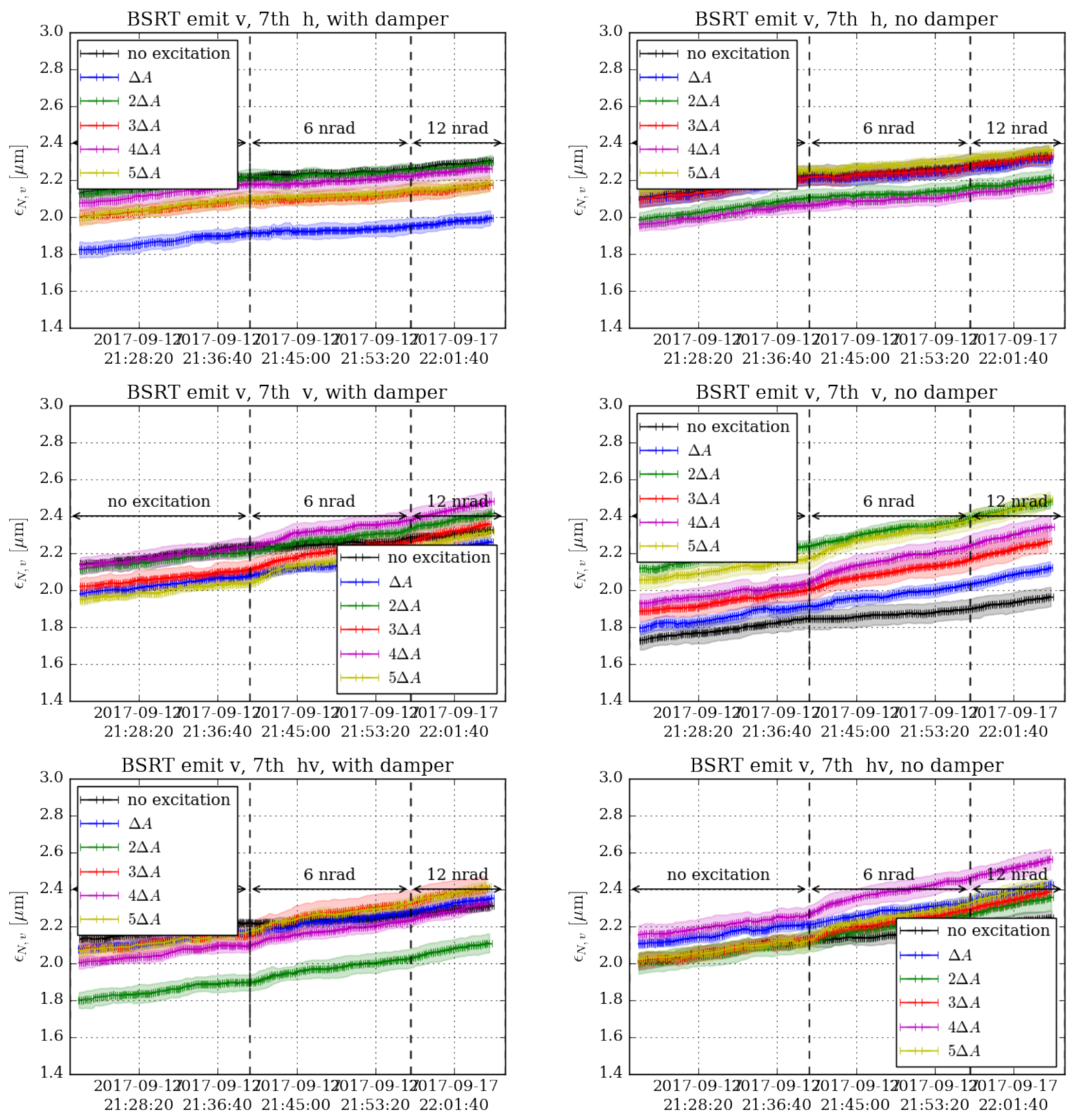

Figure 39. $7^{\text {th }}$ turn pulsing: emittance in the vertical plane measured with the BSRT (Gaussian fit) and as defined in Eqn. 22 together with the $1 \sigma$ standard deviation over the bunches with the same excitation amplitude $n \cdot \Delta A$ indicated as an envelope for each amplitude. For all emittance values a moving average over 10 time stamps is performed. 

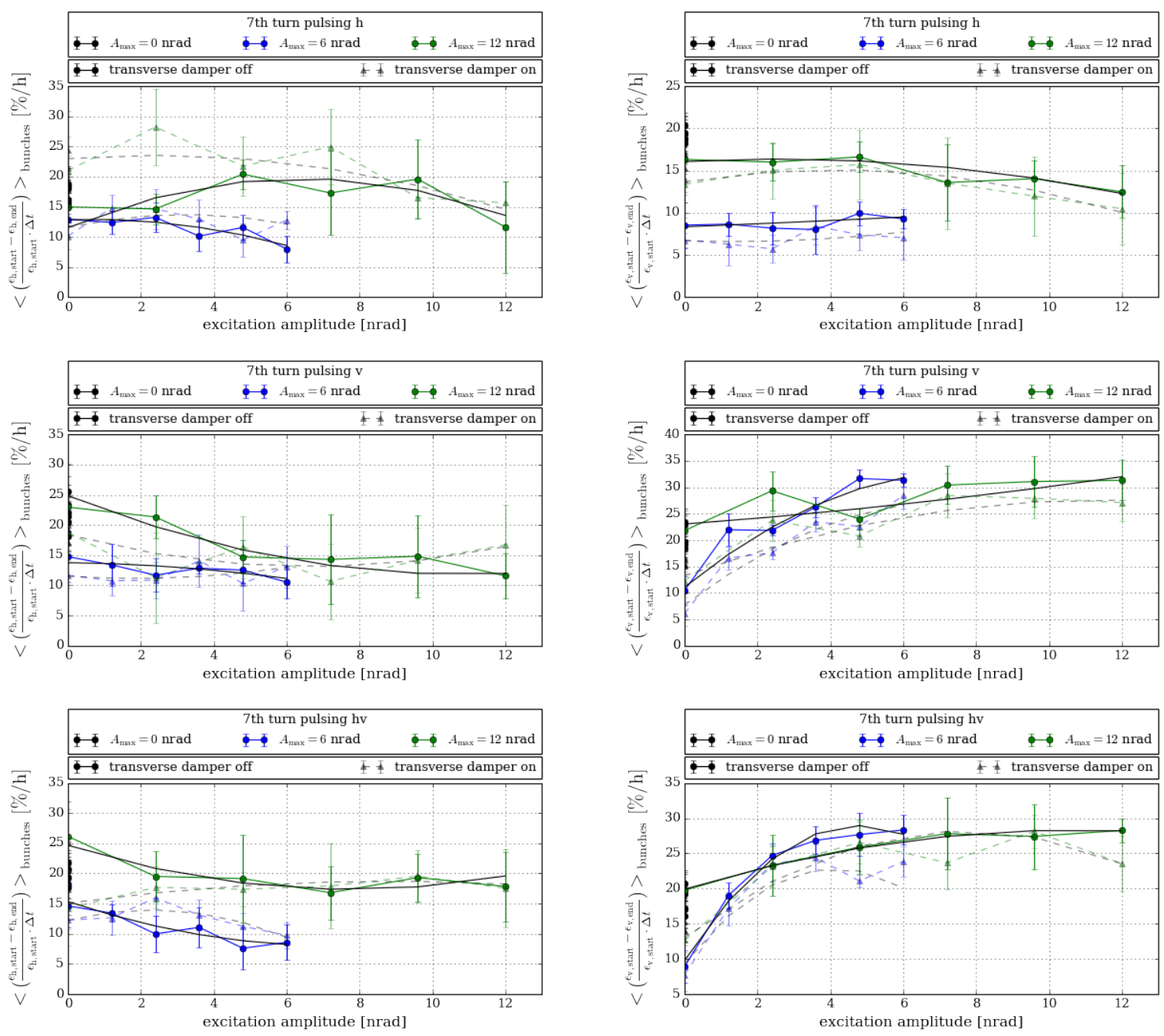

Figure 40. $7^{\text {th }}$ turn pulsing: Average emittance growth rate in the horizontal (left) and vertical (right) plane measured with the BSRT and as defined in Eqn. 25 using the emittance $\varepsilon$ from the LHC Logging database. The measurements for the different maximum excitation amplitudes $A_{\max }$ are indicated in different colors. The bunches for which the transverse damper is active are shown with solid dots and the bunches for which the transverse damper is not active are shown with slightly transparent triangles. The emittance growth rate increases approximately quadratically with the excitation amplitude $n \cdot \Delta A$, if a dependence on the excitation amplitude is observed. The quadratic fit to the emittance growth rate is indicated with a solid line for the bunches with transverse damper active and with a dashed line for the bunches with the transverse damper not active. For all emittance values a moving average over 10 time stamps is performed. 


\section{3. $7^{\text {th }}$ turn pulsing: BSRT profile analysis}

\section{a. $7^{\text {th }}$ turn pulsing: BSRT profiles - statistical parameters}


Figure $41.7^{\text {th }}$ turn pulsing: relative beam sigma calculated over the cumulative sum (Eqn. 17) in the horizontal plane measured with the BSRT together with the $1 \sigma$ standard deviation over the bunches with the same excitation amplitude $n \cdot \Delta A$ indicated as an envelope for each amplitude. For all values a moving average over 10 time stamps is performed and the average, explicitly not moving average, profiles are used. There is no dependence of the beam sigma on the excitation amplitude $n \cdot \Delta A$. 



Figure 42. $7^{\text {th }}$ turn pulsing: relative beam sigma calculated over the cumulative sum (Eqn. 17) in the vertical plane measured with the BSRT together with the $1 \sigma$ standard deviation over the bunches with the same excitation amplitude $n \cdot \Delta A$ indicated as an envelope for each amplitude. For all beam sigma values a moving average over 10 time stamps is performed and the average, explicitly not moving average, profiles are used. A clear excitation amplitude dependent emittance growth for pulsing in $\mathrm{V}$ and $\mathrm{H}+\mathrm{V}$ is visible. 

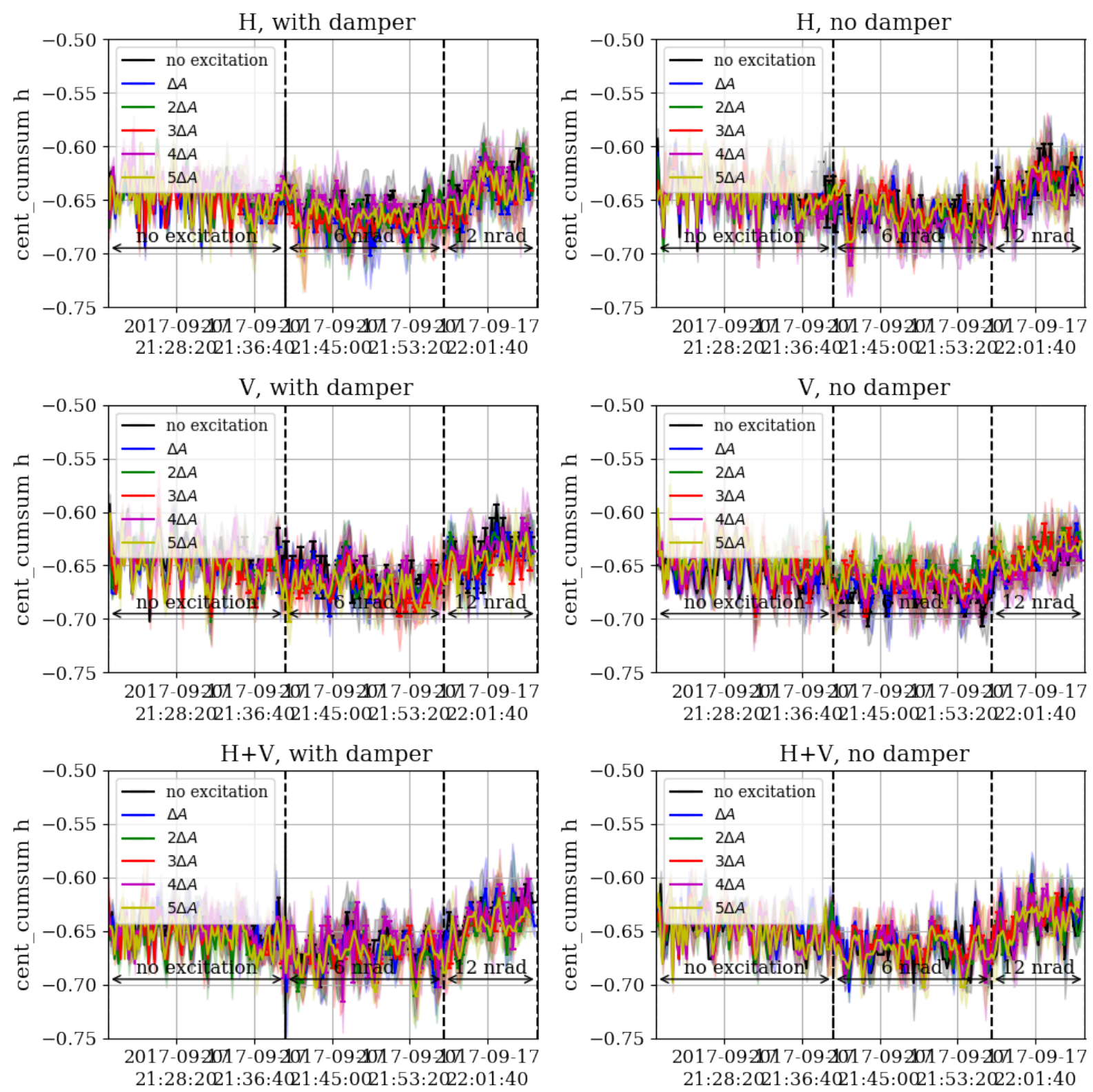

Figure 43. $7^{\text {th }}$ turn pulsing: centroid calculated over the cumulative sum (Eqn. 17) in the horizontal plane measured with the BSRT together with the $1 \sigma$ standard deviation over the bunches with the same excitation amplitude $n$. $\Delta A$ indicated as an envelope for each amplitude. No moving average is taken in order to show the un-smoothed oscillations. 



Figure 44. $7^{\text {th }}$ turn pulsing: centroid calculated over the cumulative sum (Eqn. 17) in the vertical plane measured with the BSRT together with the $1 \sigma$ standard deviation over the bunches with the same excitation amplitude $n \cdot \Delta A$ indicated as an envelope for each amplitude. No moving average is taken in order to show the un-smoothed oscillations. 
b. $7^{\text {th }}$ turn pulsing: BSRT profiles - $q$-Gaussian fit parameters in the horizontal plane
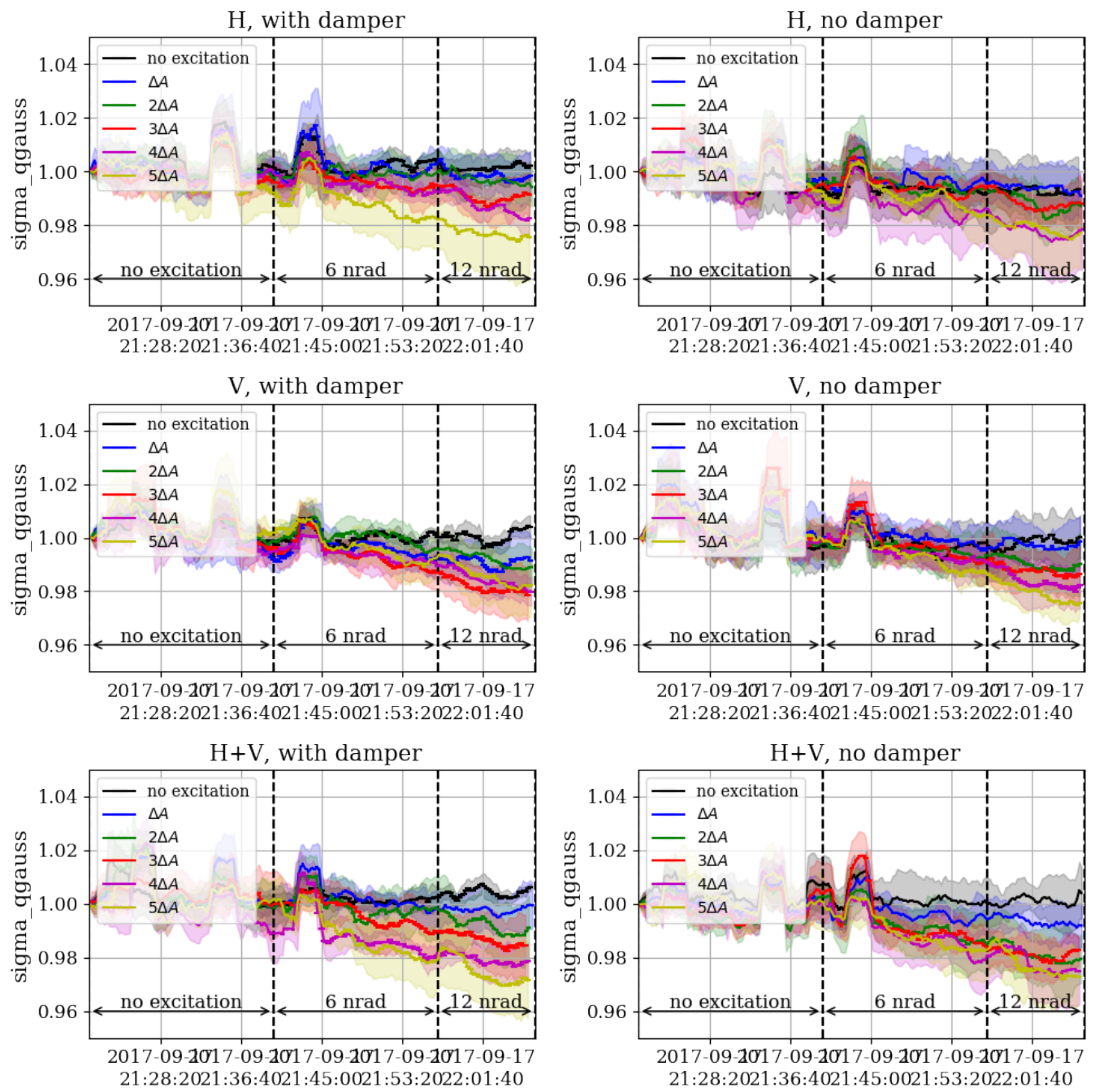

Figure 45. $7^{\text {th }}$ turn pulsing: relative beam sigma from q-Gaussian fit of the BSRT profiles (Eqn. 9) in the horizontal plane measured with the BSRT together with the $1 \sigma$ standard deviation over the bunches with the same excitation amplitude $n \cdot \Delta A$ indicated as an envelope for each amplitude. For all values the moving average profiles are used without any further averaging afterwards. 
$\mathrm{H}$, with damper


$\mathrm{H}$, no damper
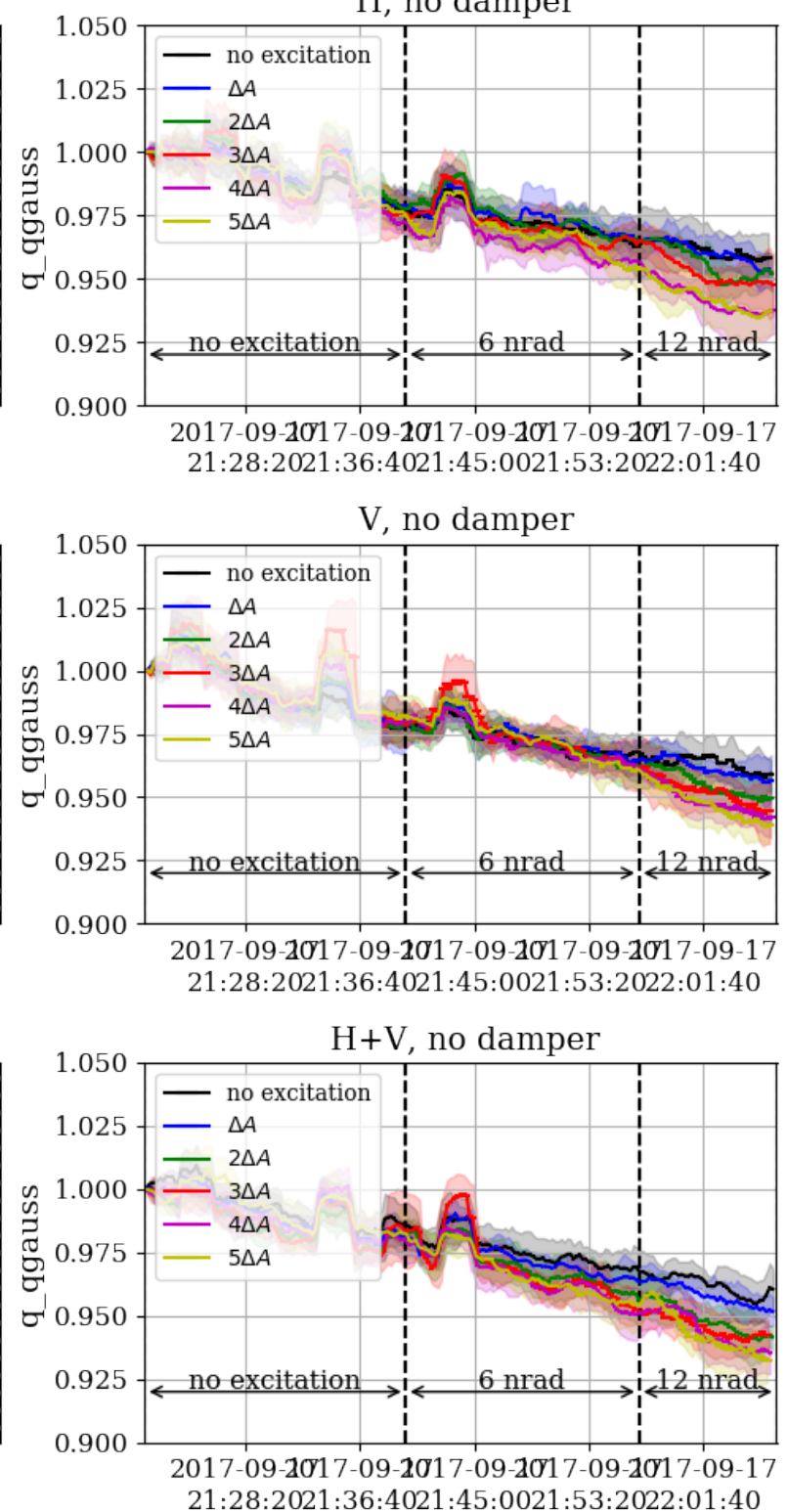

Figure 46. $7^{\text {th }}$ turn pulsing: relative fit parameter $q$ from q-Gaussian fit of the BSRT profiles (Eqn. 9) in the horizontal plane measured with the BSRT together with the $1 \sigma$ standard deviation over the bunches with the same excitation amplitude $n \cdot \Delta A$ indicated as an envelope for each amplitude. For all values the moving average profiles are used without any further averaging afterwards. 

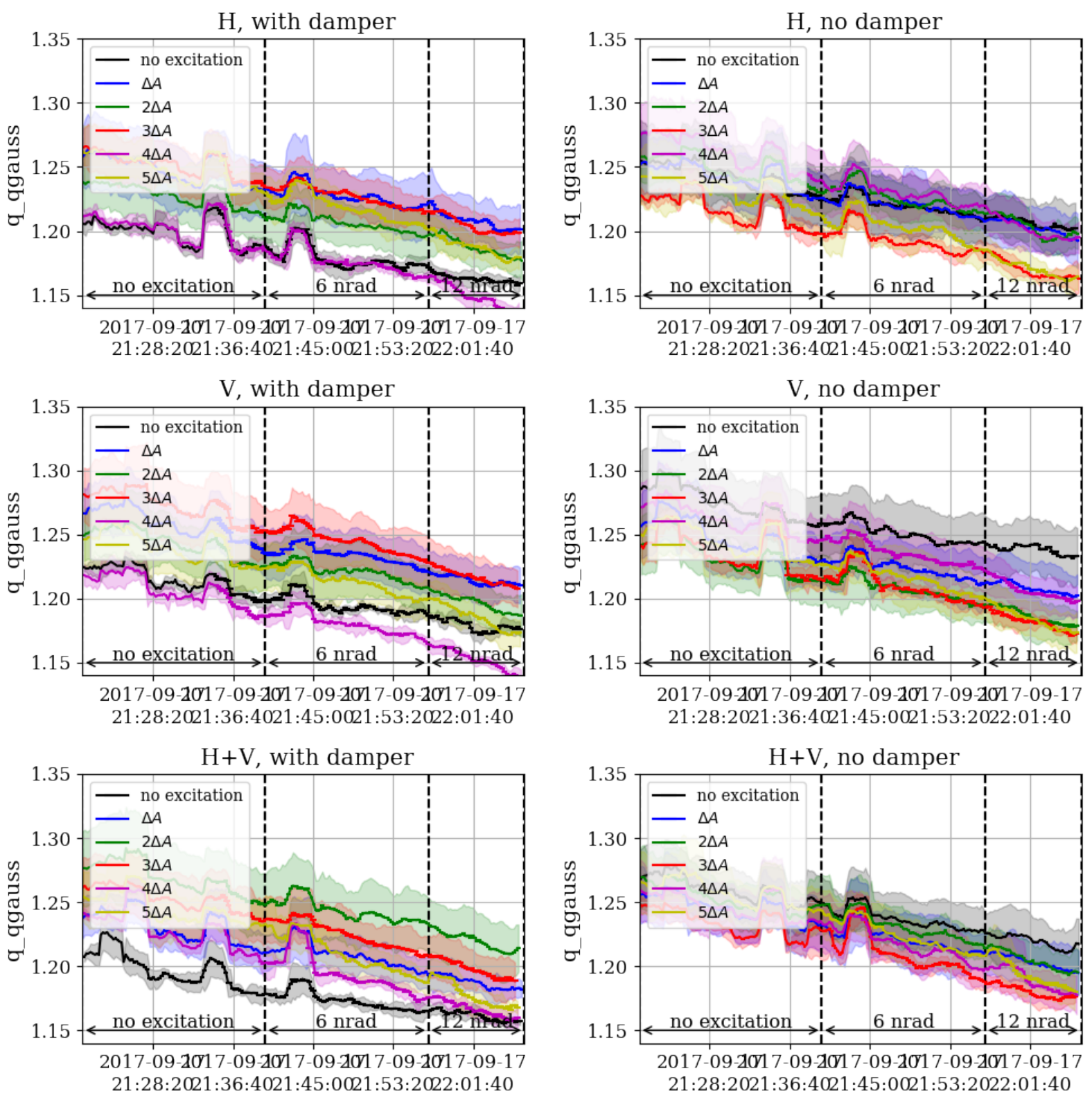

Figure 47. $7^{\text {th }}$ turn pulsing: fit parameter $q$ from q-Gaussian fit of the BSRT profiles (Eqn. 9) in the horizontal plane measured with the BSRT together with the $1 \sigma$ standard deviation over the bunches with the same excitation amplitude $n \cdot \Delta A$ indicated as an envelope for each amplitude. For all values the moving average profiles are used without any further averaging afterwards. 

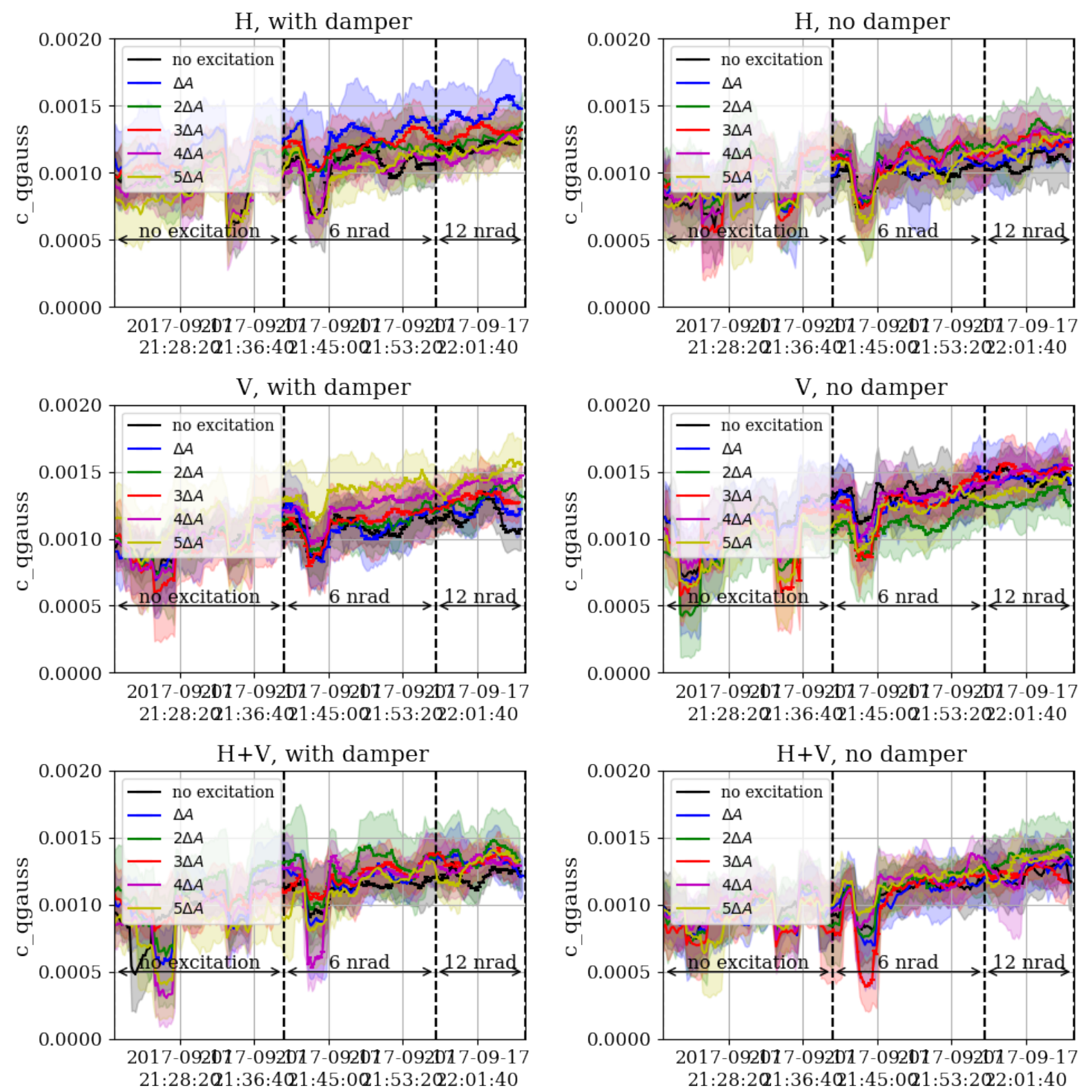

Figure 48. $7^{\text {th }}$ turn pulsing: fit parameter $c$ from q-Gaussian fit of the BSRT profiles (Eqn. 9) in the horizontal plane measured with the BSRT together with the $1 \sigma$ standard deviation over the bunches with the same excitation amplitude $n \cdot \Delta A$ indicated as an envelope for each amplitude. For all values the moving average profiles are used without any further averaging afterwards. 
$\mathrm{H}$, with damper


$\mathrm{H}$, no damper
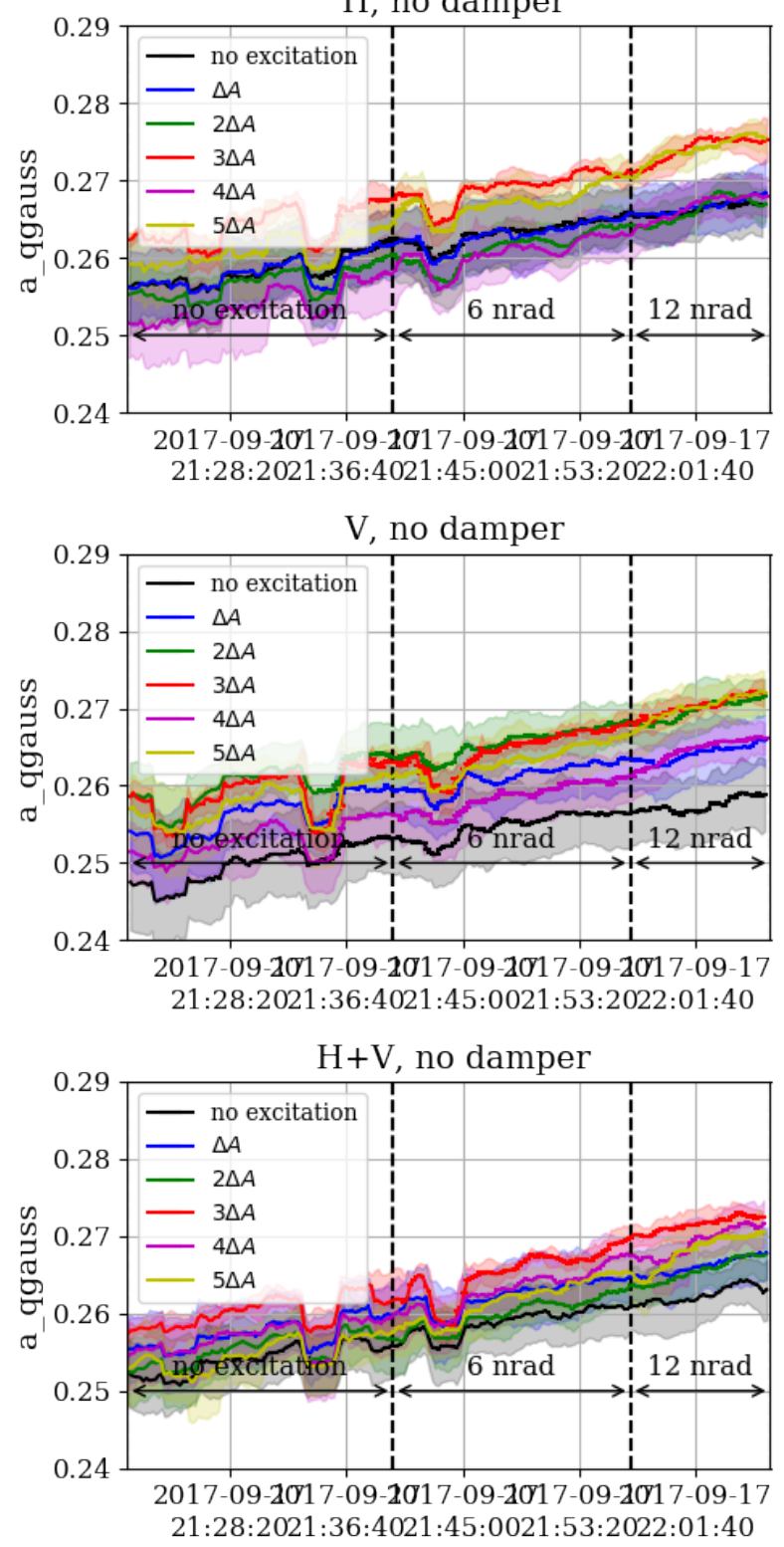

Figure 49. $7^{\text {th }}$ turn pulsing: fit parameter $a$ from q-Gaussian fit of the BSRT profiles (Eqn. 9) in the horizontal plane measured with the BSRT together with the $1 \sigma$ standard deviation over the bunches with the same excitation amplitude $n \cdot \Delta A$ indicated as an envelope for each amplitude. For all values the moving average profiles are used without any further averaging afterwards. 



Figure 50. $7^{\text {th }}$ turn pulsing: normalized $\chi^{2}$ of q-Gaussian fit of the BSRT profiles (Eqn. 9) in the horizontal plane measured with the BSRT together with the $1 \sigma$ standard deviation over the bunches with the same excitation amplitude $n \cdot \Delta A$ indicated as an envelope for each amplitude. For all values the moving average profiles are used without any further averaging afterwards. The $\chi^{2}$ stays comparably constant indicating that the quality of the fit also stays unchanged during the entire excitation period. 
c. $7^{\text {th }}$ turn pulsing: BSRT profiles - $q$-Gaussian fit parameters in the vertical plane


Figure 51. $7^{\text {th }}$ turn pulsing: relative beam sigma from q-Gaussian fit of the BSRT profiles (Eqn. 9) in the vertical plane measured with the BSRT together with the $1 \sigma$ standard deviation over the bunches with the same excitation amplitude $n \cdot \Delta A$ indicated as an envelope for each amplitude. For all values the moving average profiles are used without any further averaging afterwards. 



Figure 52. $7^{\text {th }}$ turn pulsing: relative fit parameter $q$ from q-Gaussian fit of the BSRT profiles (Eqn. 9) in the vertical plane measured with the BSRT together with the $1 \sigma$ standard deviation over the bunches with the same excitation amplitude $n \cdot \Delta A$ indicated as an envelope for each amplitude. For all values the moving average profiles are used without any further averaging afterwards. 

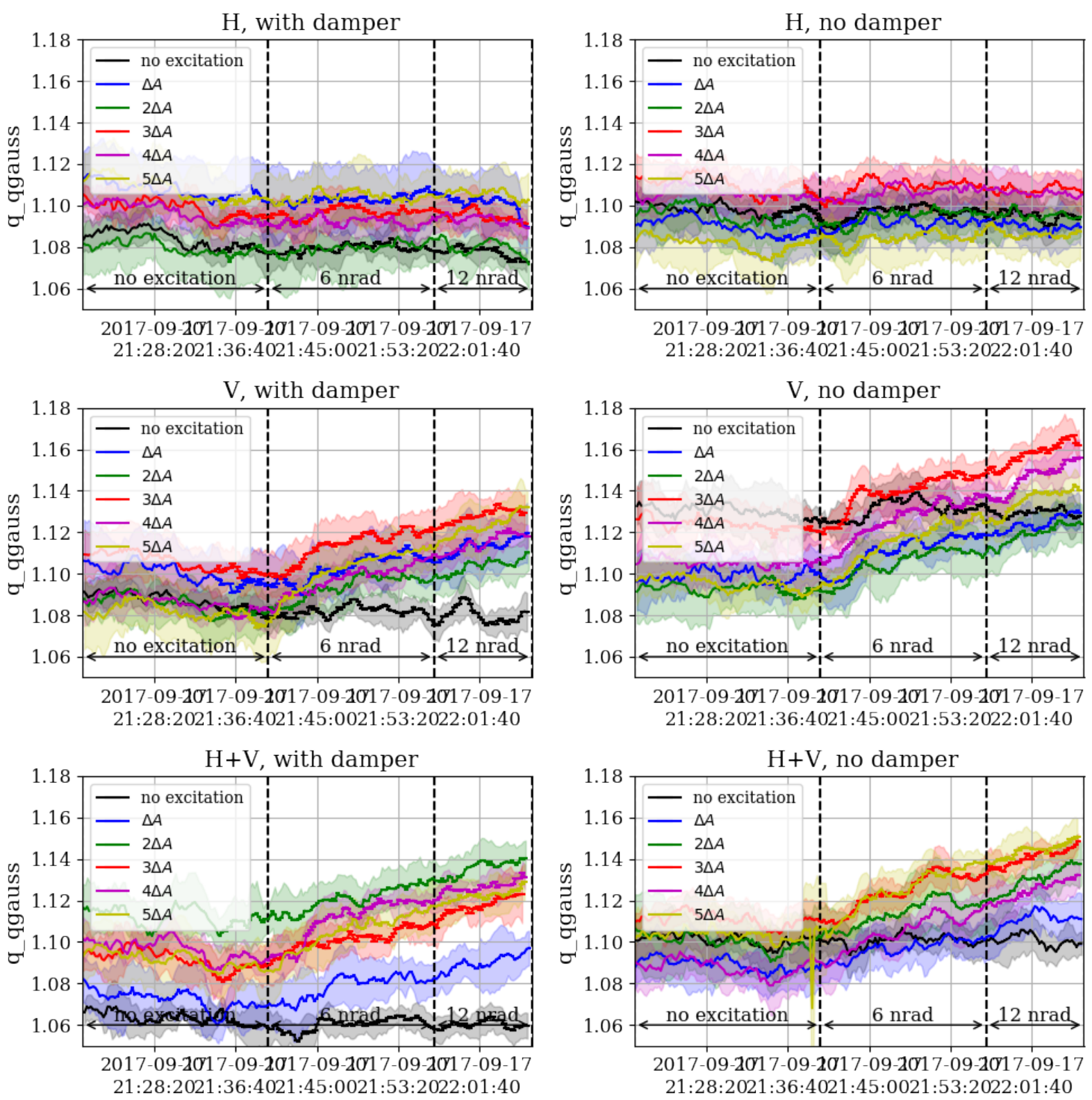

Figure 53. $7^{\text {th }}$ turn pulsing: fit parameter $q$ from q-Gaussian fit of the BSRT profiles (Eqn. 9) in the vertical plane measured with the BSRT together with the $1 \sigma$ standard deviation over the bunches with the same excitation amplitude $n \cdot \Delta A$ indicated as an envelope for each amplitude. For all values the moving average profiles are used without any further averaging afterwards. 

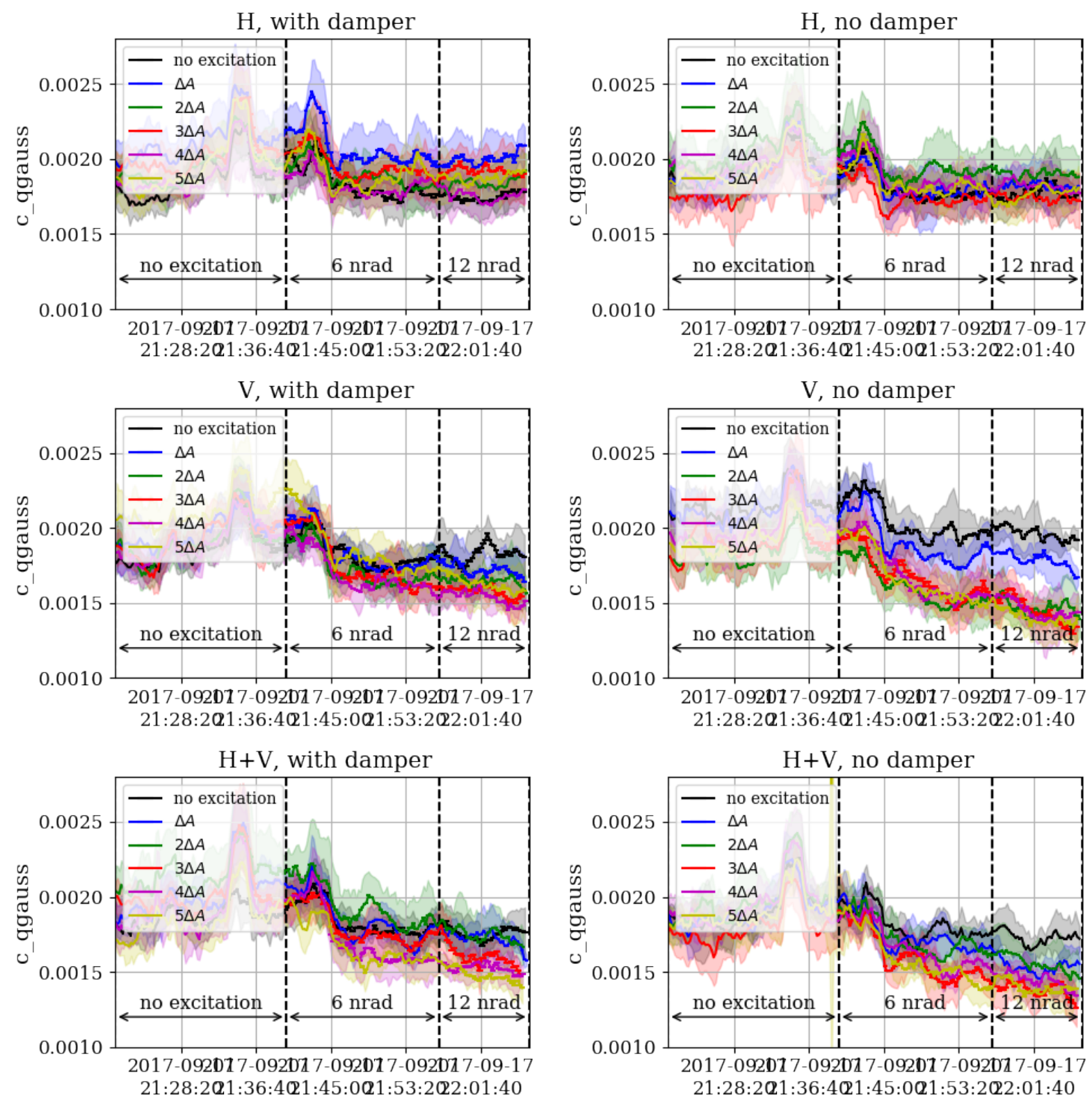

Figure 54. $7^{\text {th }}$ turn pulsing: fit parameter $c$ from q-Gaussian fit of the BSRT profiles (Eqn. 9) in the vertical plane measured with the BSRT together with the $1 \sigma$ standard deviation over the bunches with the same excitation amplitude $n \cdot \Delta A$ indicated as an envelope for each amplitude. For all values the moving average profiles are used without any further averaging afterwards. 

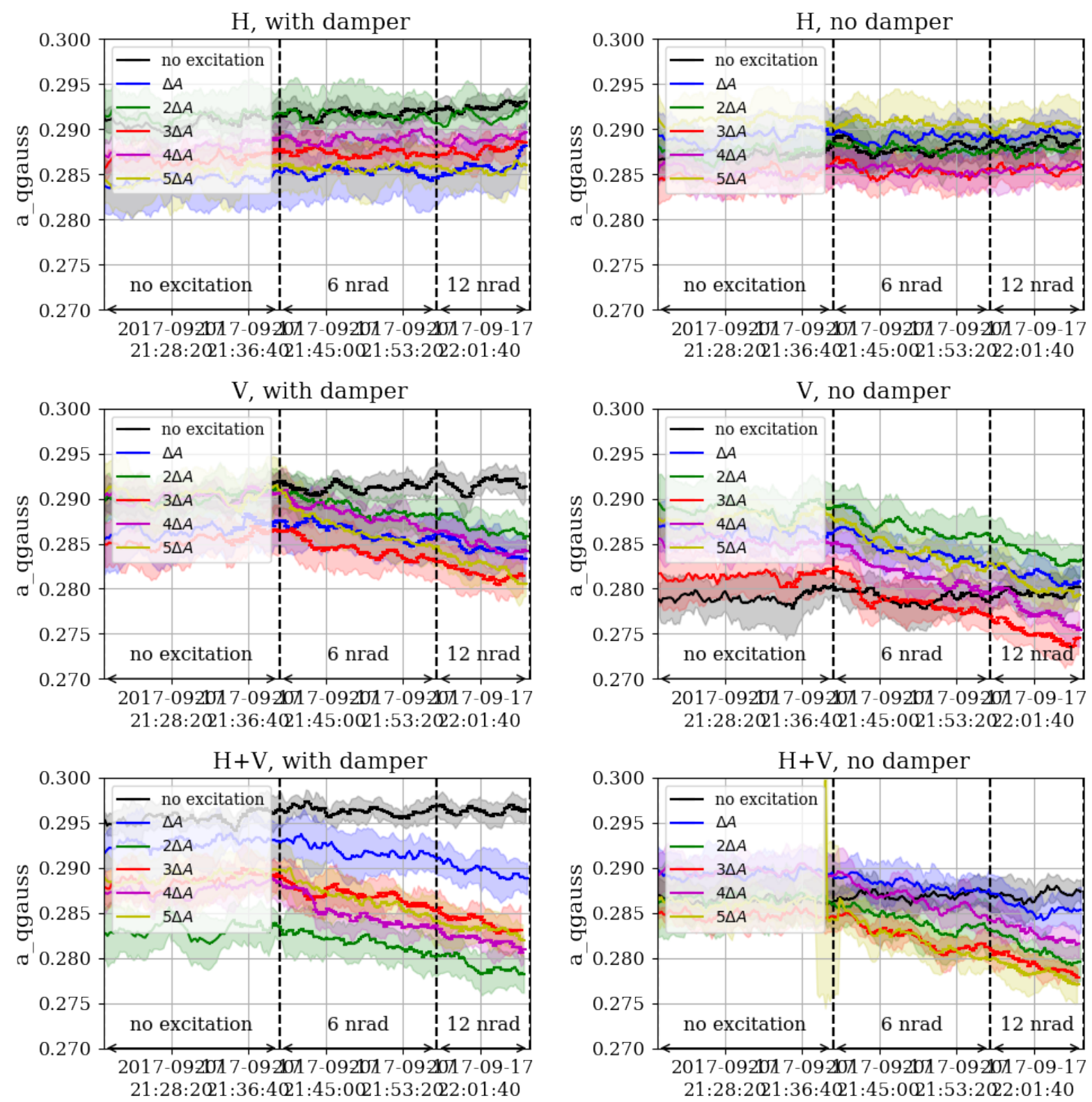

Figure 55. $7^{\text {th }}$ turn pulsing: fit parameter $a$ from q-Gaussian fit of the BSRT profiles (Eqn. 9) in the vertical plane measured with the BSRT together with the $1 \sigma$ standard deviation over the bunches with the same excitation amplitude $n \cdot \Delta A$ indicated as an envelope for each amplitude. For all values the moving average profiles are used without any further averaging afterwards. 

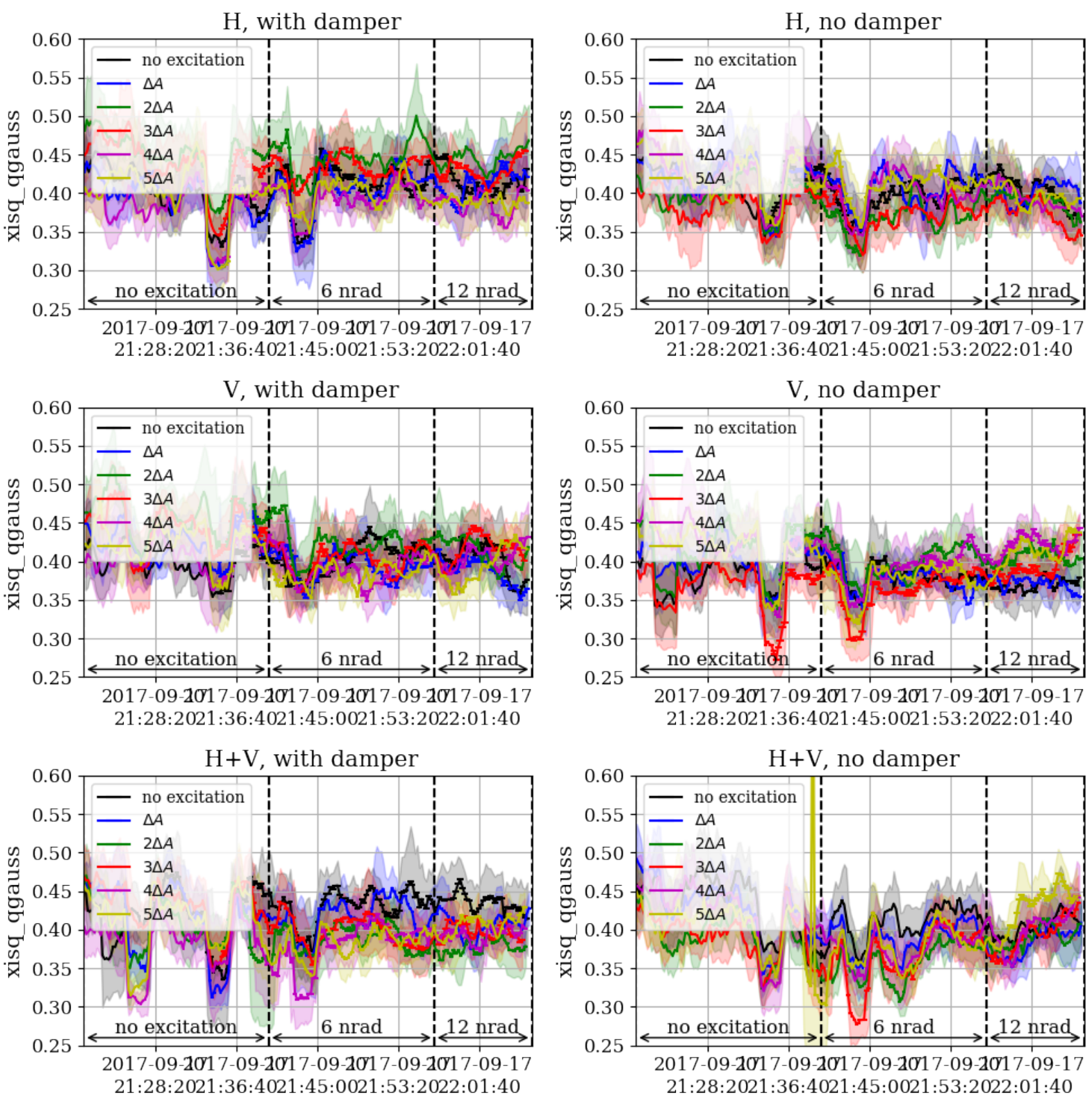

Figure 56. $7^{\text {th }}$ turn pulsing: normalized $\chi^{2}$ of q-Gaussian fit of the BSRT profiles (Eqn. 9) in the vertical plane measured with the BSRT together with the $1 \sigma$ standard deviation over the bunches with the same excitation amplitude $n \cdot \Delta A$ indicated as an envelope for each amplitude. For all values the moving average profiles are used without any further averaging afterwards. The $\chi^{2}$ stays comparably constant indicating that the quality of the fit also stays unchanged during the entire excitation period. 
d. $7^{\text {th }}$ turn pulsing: BSRT profiles of representative bunches

no damper, no excitation

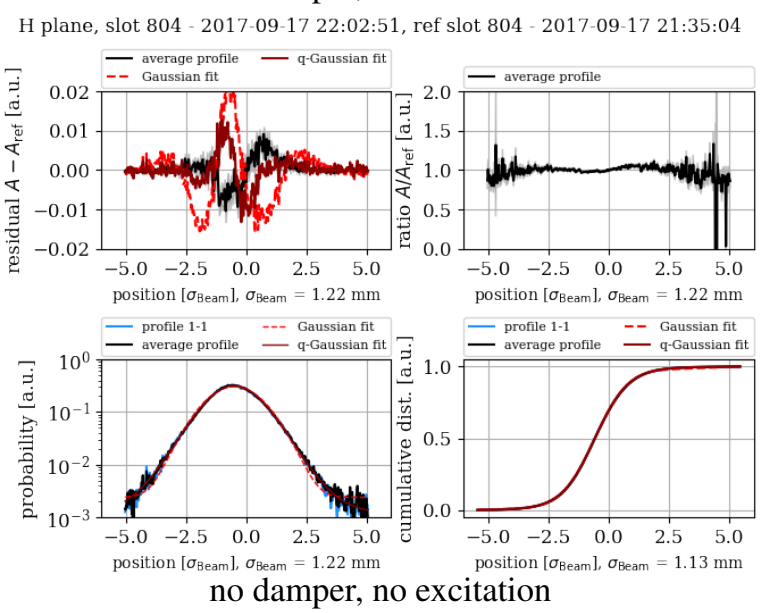

V plane, slot 804 - 2017-09-17 22:02:51, ref slot 804 - 2017-09-17 21:35:04
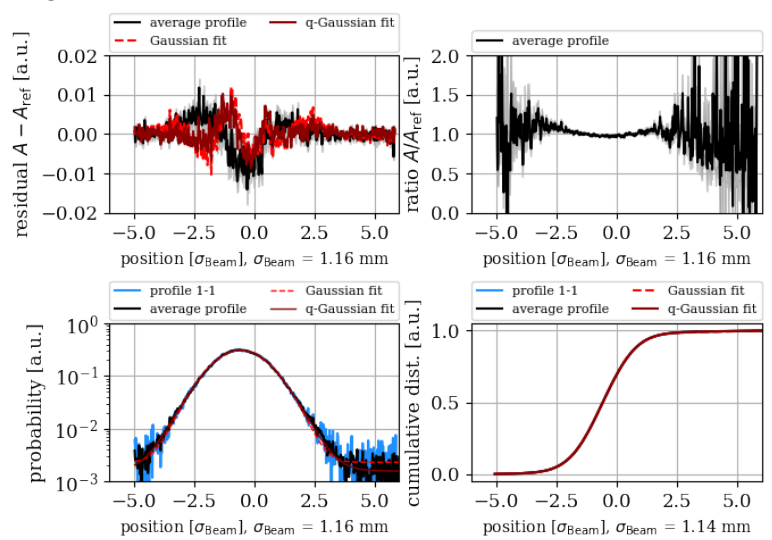

no damper, with excitation $(5 \cdot \Delta A)$ in $\mathrm{H}$
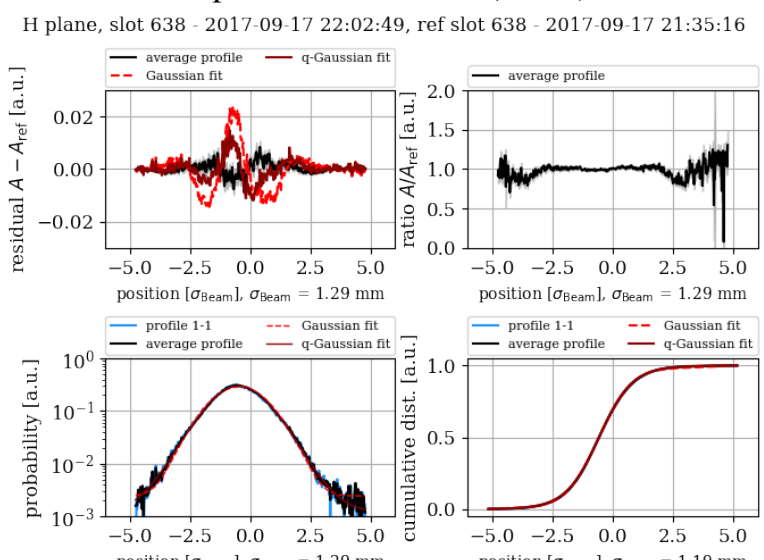

position [ $\sigma_{\text {Beam }],} \sigma_{\text {Beam }}=1.29 \mathrm{~mm}$ no damper, with excitation $(5 \cdot \Delta A)$ in $\mathrm{H}$
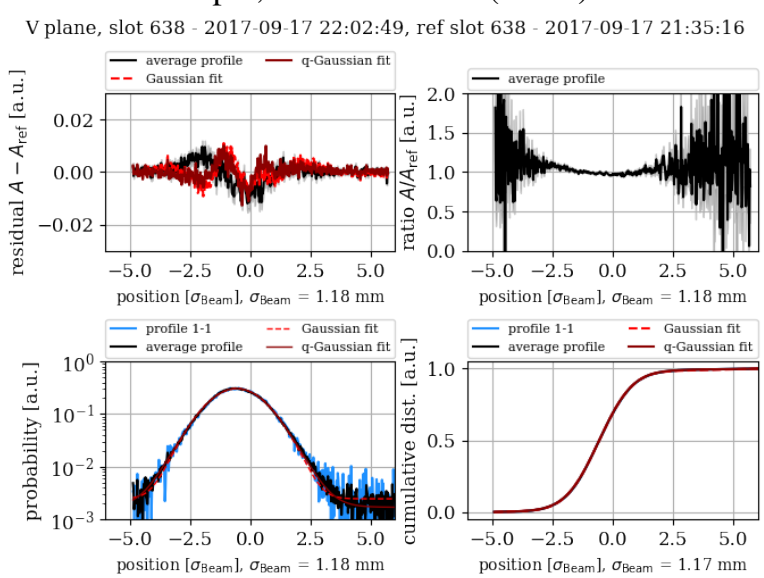

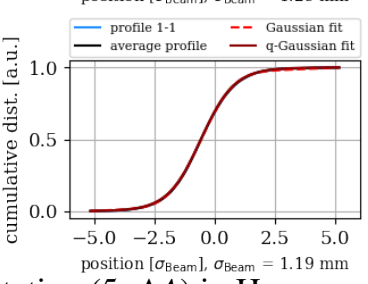

position [ $\left.\sigma_{\text {Beam }}\right], \sigma_{\text {Beam }}=1.18 \mathrm{~mm}$

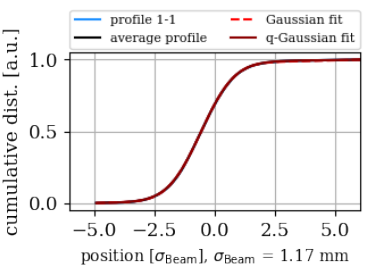

Figure 57. $7^{\text {th }}$ turn pulsing: BSRT profile in the horizontal (top) and vertical (bottom) plane of one bunch without resonant excitation (left) and one bunch experiencing the maximum excitation (right) for an excitation only in H. For all bunches the damper is not active. The residual is taken in respect to the start (2017-09-17 22:03) and end (201709-17 21:35) of the $7^{\text {th }}$ turns pulsing with a few minutes subtracted in order to account for the moving average of the profiles over 11 time stamps. The $\mathrm{x}$-axis is the position of the BSRT image projection and not the proton beam sigma. 
no damper, no excitation

H plane, slot 1698 - 2017-09-17 22:02:56, ref slot 1698 - 2017-09-17 21:35:08
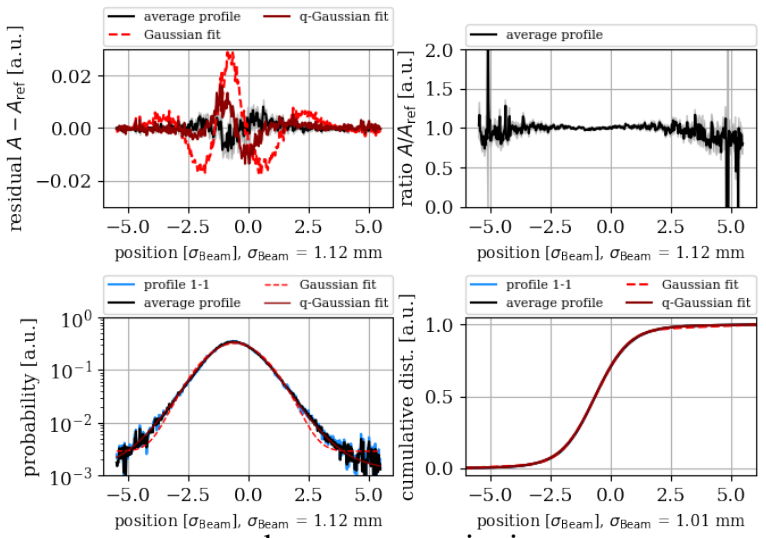
no damper, no excitation

V plane, slot 1698 - 2017-09-17 22:02:56, ref slot 1698 - 2017-09-17 21:35:08
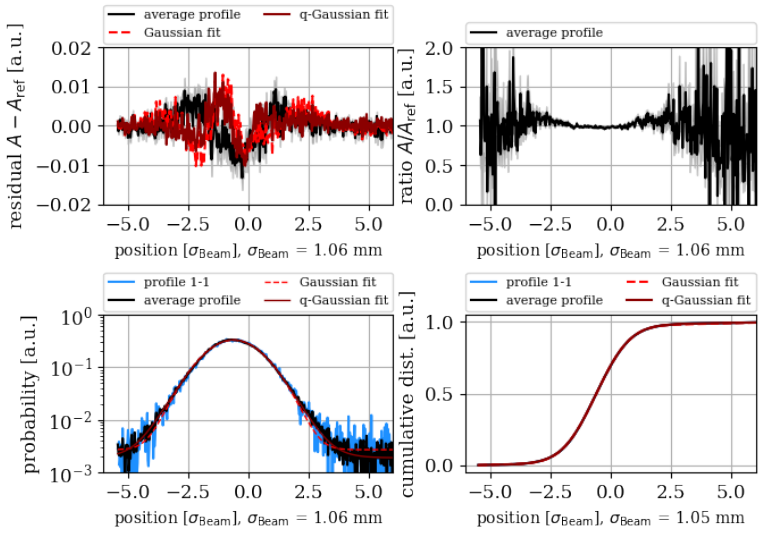
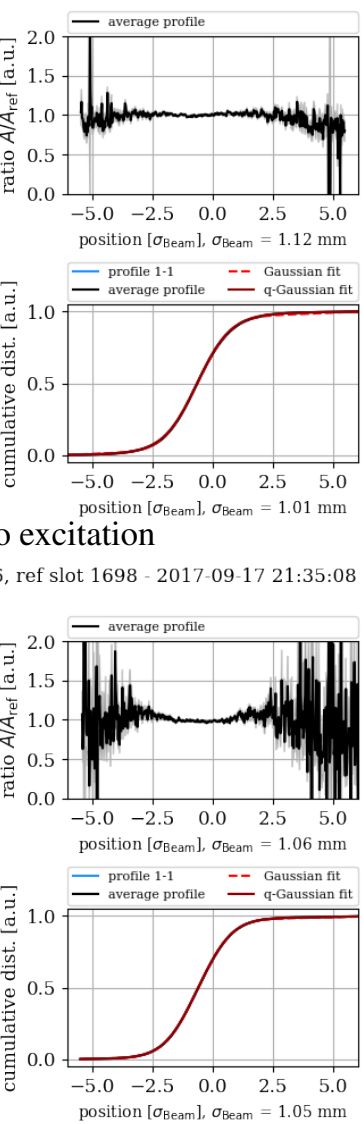

no damper, with excitation $(5 \cdot \Delta A)$ in $\mathrm{V}$

H plane, slot 1532 - 2017-09-17 22:02:55, ref slot 1532 - 2017-09-17 21:35:06
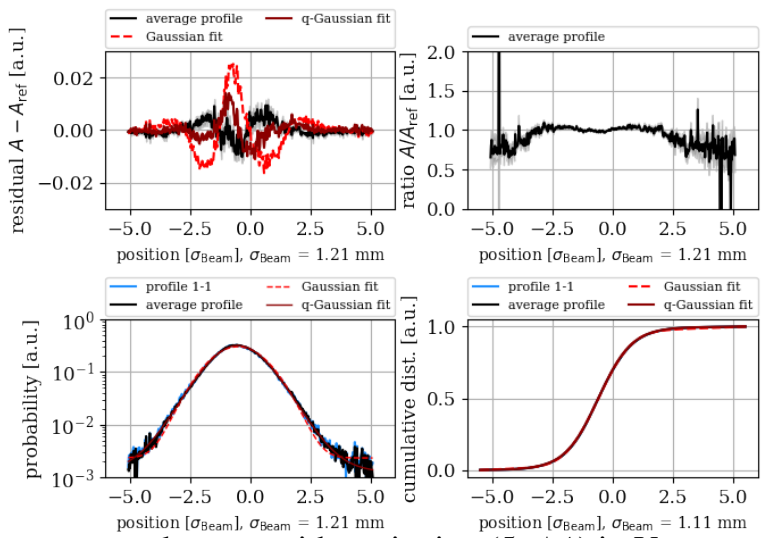

position $\left[\sigma_{\text {Beam }}\right], \sigma_{\text {Bea }}=1.21 \mathrm{~mm}$ position $\left[\sigma_{\text {Beam }}\right], \sigma_{\text {Beam }}=1.11 \mathrm{~mm}$ no damper, with excitation $(5 \cdot \Delta A)$ in $\mathrm{V}$

V plane, slot 1532 - 2017-09-17 22:02:55, ref slot 1532 - 2017-09-17 21:35:06
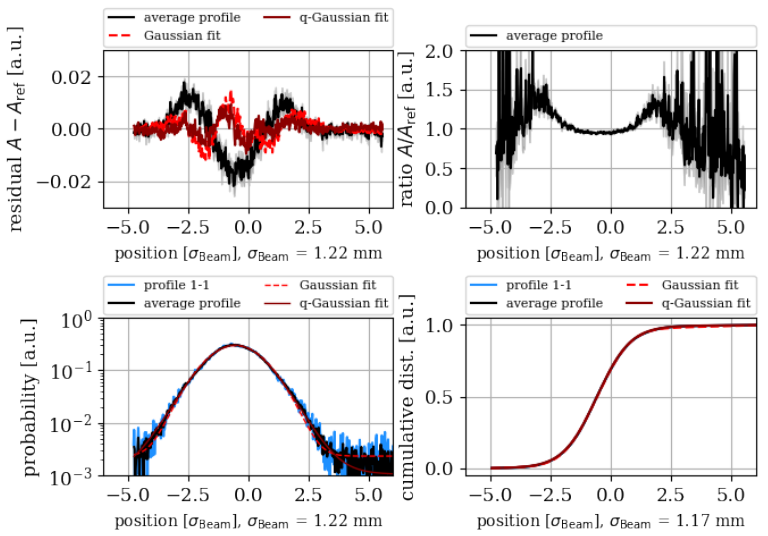

Figure 58. $7^{\text {th }}$ turn pulsing: BSRT profile in the horizontal (top) and vertical (bottom) plane of one bunch without resonant excitation (left) and one bunch experiencing the maximum excitation (right) for an excitation only in V. For all bunches the damper is not active. The residual is taken in respect to the start (2017-09-17 22:03) and end (201709-17 21:35) of the $7^{\text {th }}$ turn pulsing with a few minutes subtracted in order to account for the moving average of the profiles over 11 time stamps. Note that 22:56 is also after the closed orbit shift in the vertical plane. The $\mathrm{x}$-axis is the position of the BSRT image projection and not the proton beam sigma. 
no damper, no excitation


no damper, no excitation

V plane, slot 2862 - 2017-09-17 22:02:46, ref slot 2862 - 2017-09-17 21:35:01
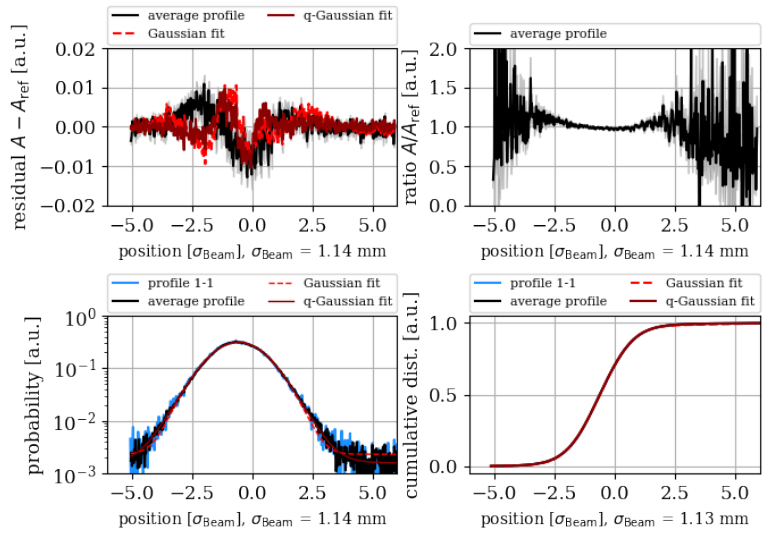


no damper, with excitation $(5 \cdot \Delta A)$ in $\mathrm{H}+\mathrm{V}$

H plane, slot 2696 - 2017-09-17 22:02:59, ref slot 2696 - 2017-09-17 21:35:00
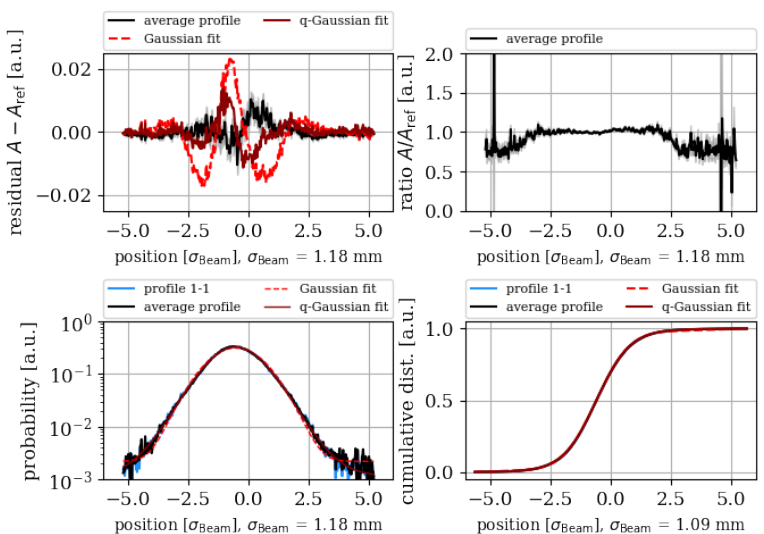
position $\left[\sigma_{\text {Beam }}\right], \sigma_{\text {Beam }}=1.18 \mathrm{~mm}$
no damper, with excitation $(5 \cdot \Delta A)$ in $\mathrm{H}+\mathrm{V}$

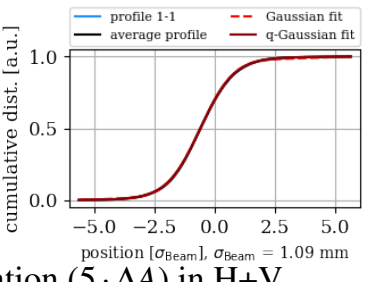

V plane, slot 2696 - 2017-09-17 22:02:59, ref slot 2696 - 2017-09-17 21:35:00


Figure 59. $7^{\text {th }}$ turn pulsing: BSRT profile in the horizontal (top) and vertical (bottom) plane of one bunch without resonant excitation (left) and one bunch experiencing the maximum excitation (right) for an excitation only in $\mathrm{H}+\mathrm{V}$. For all bunches the damper is not active. The residual is taken in respect to the start (2017-09-17 22:03) and end (2017-09-17 21:35) of the $7^{\text {th }}$ turn pulsing with a few minutes subtracted in order to account for the moving average of the profiles over 11 time stamps. Note that 22:56 is also after the closed orbit shift in the vertical plane. The $\mathrm{x}$-axis is the position of the BSRT image projection and not the proton beam sigma. 
with damper, no excitation

H plane, slot 442 - 2017-09-17 23:22:52, ref slot 442 - 2017-09-17 22:57:11
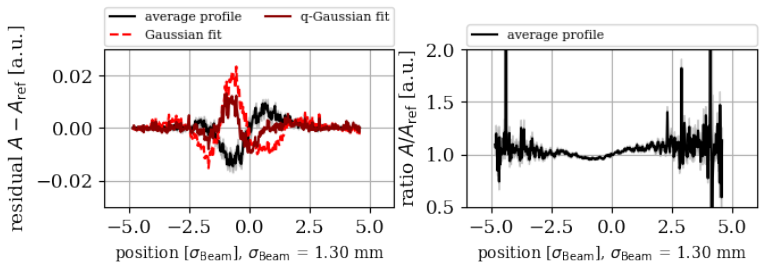

- profile 1-1 --- Gaussian fit

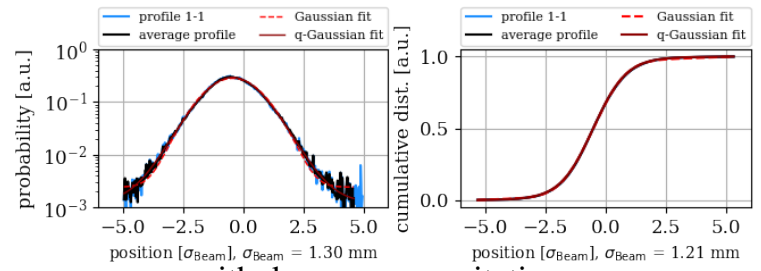

with damper, no excitation
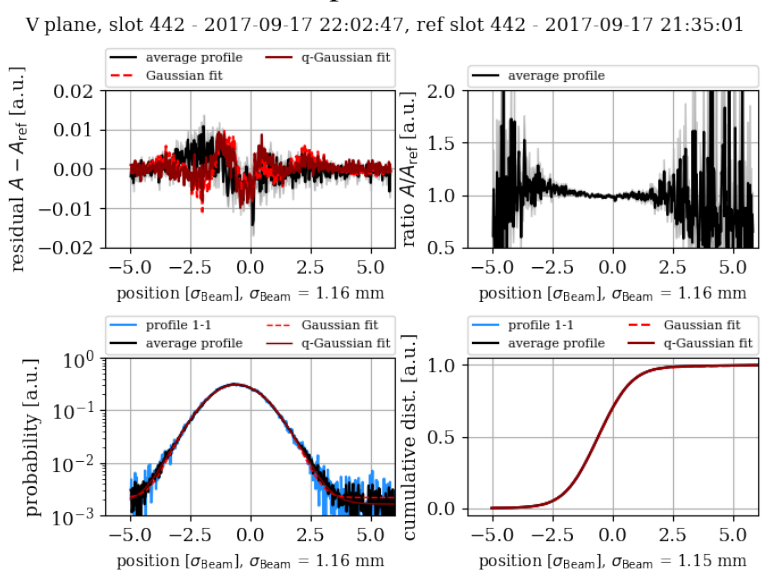

with damper, with excitation $(5 \cdot \Delta A)$ in $\mathrm{H}$

H plane, slot 608 - 2017-09-17 22:02:49, ref slot 608 - 2017-09-17 21:35:15
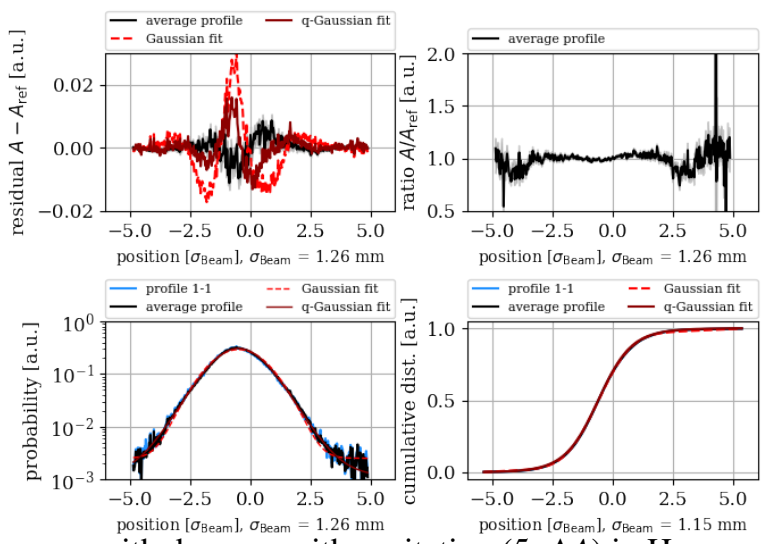

with damper, with excitation $(5 \cdot \Delta A)$ in $\mathrm{H}$

V plane, slot 608 - 2017-09-17 22:02:49, ref slot 608 - 2017-09-17 21:35:15
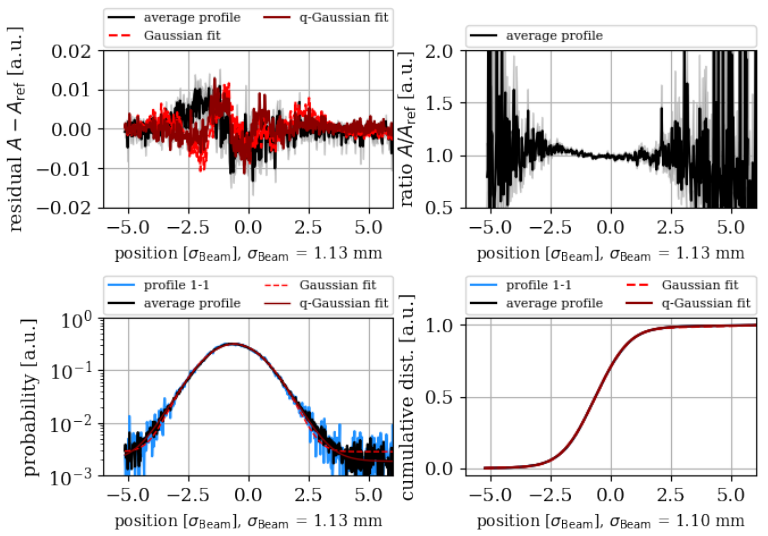

Figure 60. $7^{\text {th }}$ turn pulsing: BSRT profile in the horizontal (top) and vertical (bottom) plane of one bunch without resonant excitation (left) and one bunch experiencing the maximum excitation (right) for an excitation only in H. For all bunches the damper is active. The residual is taken in respect to the start (2017-09-17 22:03) and end (2017-09-17 21:35) of the $7^{\text {th }}$ turn pulsing with a few minutes subtracted in order to account for the moving average of the profiles over 11 time stamps. The $\mathrm{x}$-axis is the position of the BSRT image projection and not the proton beam sigma. 
with damper, no excitation

H plane, slot 1336 - 2017-09-17 22:02:51, ref slot 1336 - 2017-09-17 21:35:04
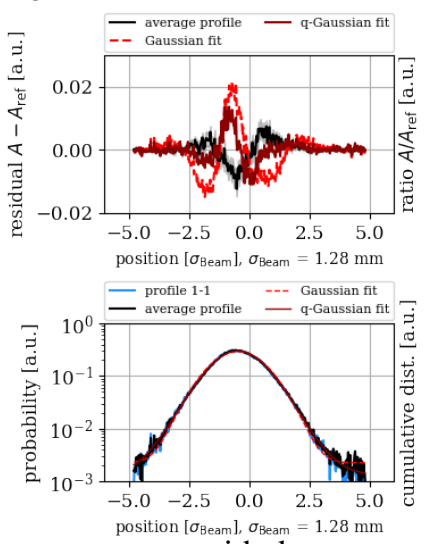

with damper, no excitation

V plane, slot 1336 - 2017-09-17 22:02:51, ref slot 1336 - 2017-09-17 21:35:04
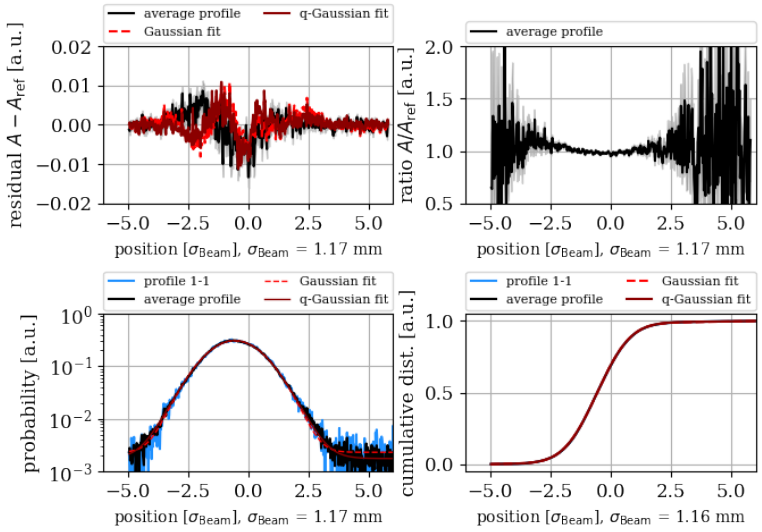


with damper, with excitation $(5 \cdot \Delta A)$ in $\mathrm{V}$

H plane, slot 1502 - 2017-09-17 22:02:53, ref slot 1502 - 2017-09-17 21:35:06
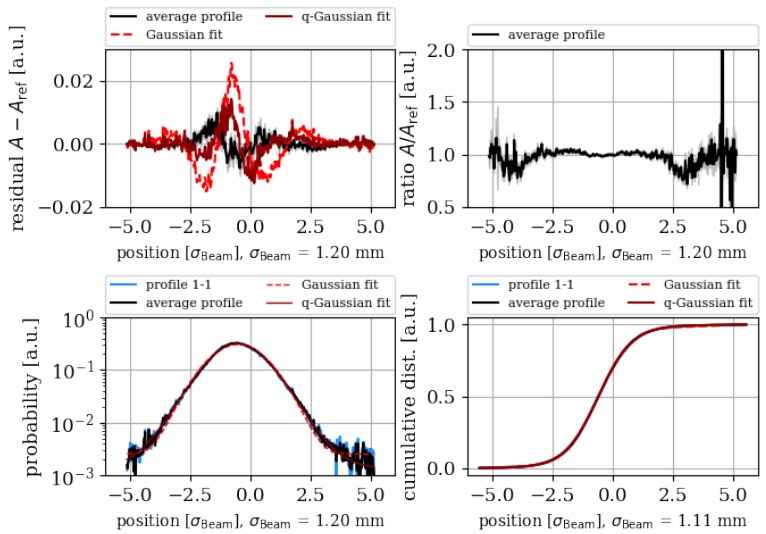
position $\left[\sigma_{\text {Beam }}\right], \sigma_{\text {Beam }}=1.20 \mathrm{~mm}$
with damper, with excitation $(5 \cdot \Delta A)$ in $\mathrm{V}$

V plane, slot 1502 - 2017-09-17 22:02:53, ref slot 1502 - 2017-09-17 21:35:06
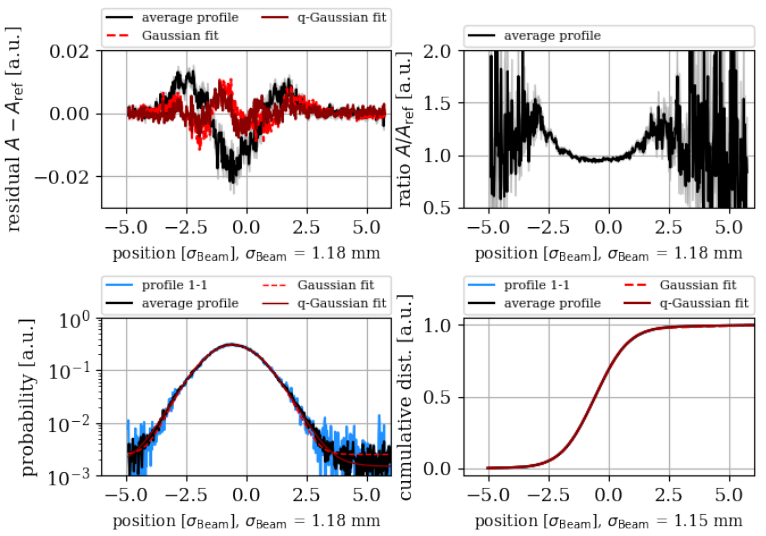

Figure 61. $7^{\text {th }}$ turn pulsing: BSRT profile in the horizontal (top) and vertical (bottom) plane of one bunch without resonant excitation (left) and one bunch experiencing the maximum excitation (right) for an excitation only in V. For all bunches the damper is active. The residual is taken in respect to the start (2017-09-17 22:03) and end (2017-09-17 21:35) of the $7^{\text {th }}$ turn pulsing with a few minutes subtracted in order to account for the moving average of the profiles over 11 time stamps. Note that 22:56 is also after the closed orbit shift in the vertical plane. The $\mathrm{x}$-axis is the position of the BSRT image projection and not the proton beam sigma. 
with damper, no excitation

H plane, slot 2500 - 2017-09-17 22:02:57, ref slot 2500 - 2017-09-17 21:35:08
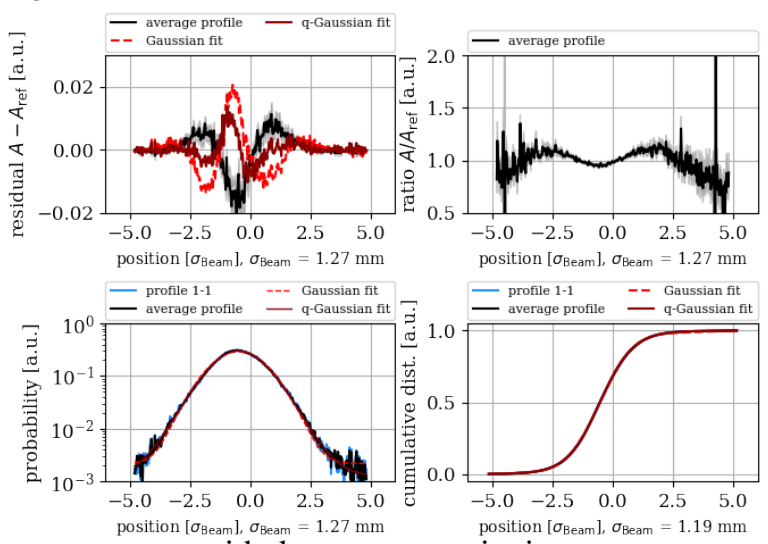

with damper, no excitation

V plane, slot 2500 - 2017-09-17 22:02:57, ref slot 2500 - 2017-09-17 21:35:08

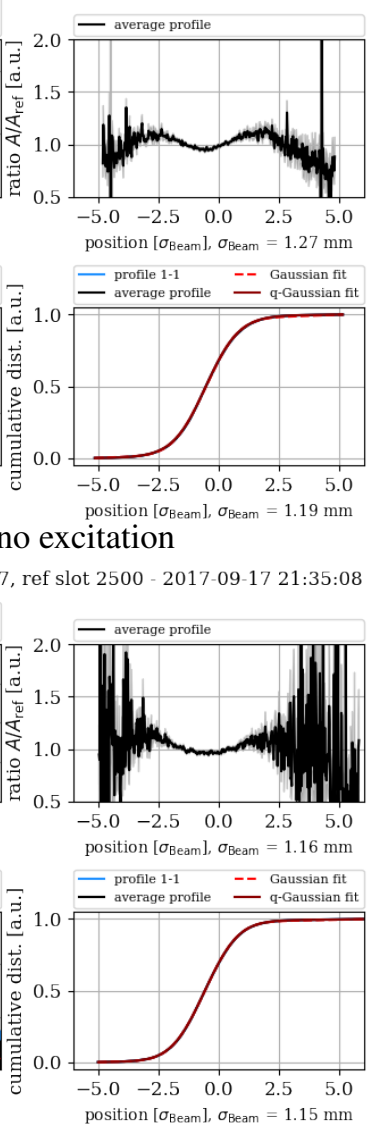

with damper, with excitation $(5 \cdot \Delta A)$ in $\mathrm{H}+\mathrm{V}$

H plane, slot 2666 - 2017-09-17 22:02:59, ref slot 2666 - 2017-09-17 21:35:00
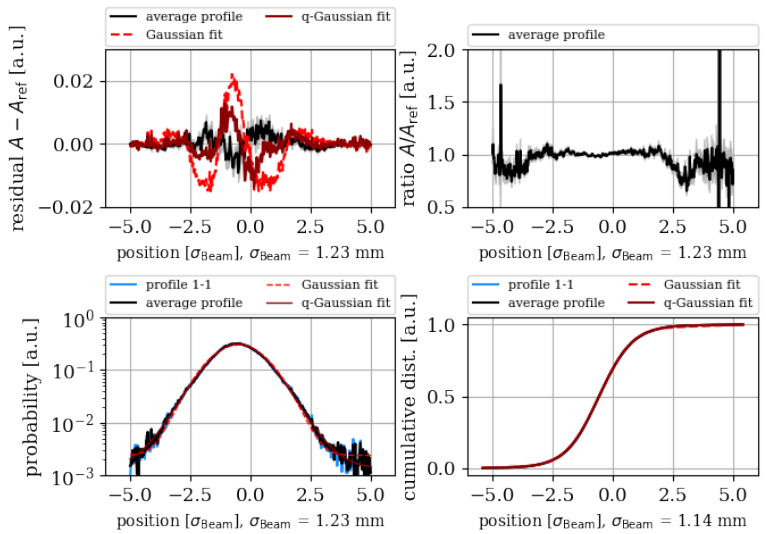

with damper, with excitation $(5 \cdot \Delta A)$ in $\mathrm{H}+\mathrm{V}$

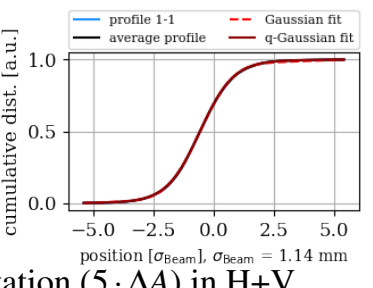

V plane, slot 2666 - 2017-09-17 22:02:59, ref slot 2666 - 2017-09-17 21:35:00


Figure 62. $7^{\text {th }}$ turn pulsing: BSRT profile in the horizontal (top) and vertical (bottom) plane of one bunch without resonant excitation (left) and one bunch experiencing the maximum excitation (right) for an excitation only in $\mathrm{H}+\mathrm{V}$. For all bunches the damper is active. The residual is taken in respect to the start (2017-09-17 22:03) and end (201709-17 21:35) of the $7^{\text {th }}$ turn pulsing with a few minutes subtracted in order to account for the moving average of the profiles over 11 time stamps. The x-axis is the position of the BSRT image projection and not the proton beam sigma. 


\section{4. $7^{\text {th }}$ turn pulsing: FMA Analysis for 2017 optics and tune}
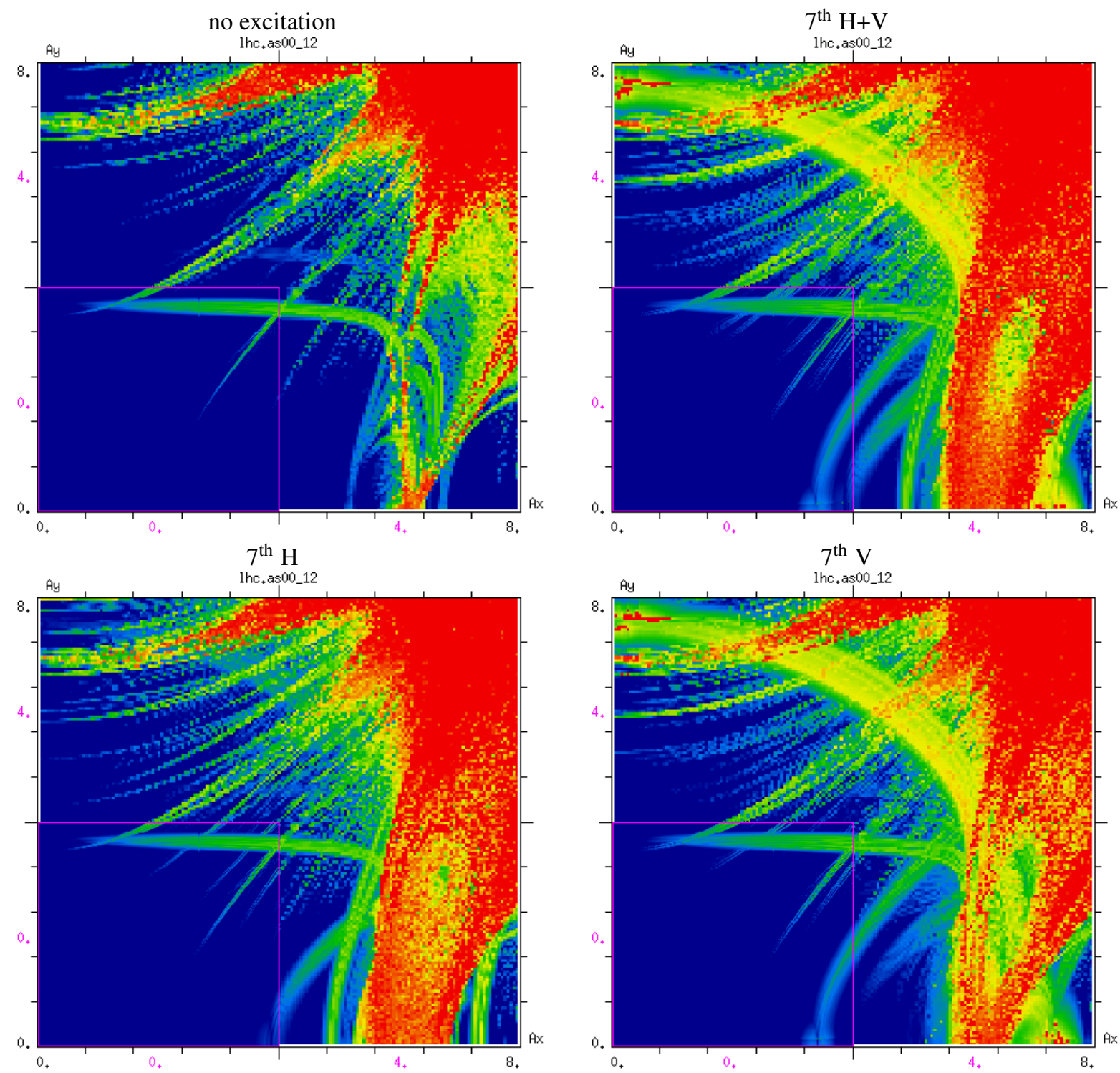

Figure 63. FMA analysis in amplitude space for $7^{\text {th }}$ turn pulsing based on the 2017 injection optics with no machine errors and a tune of $(62.27,60.295)$. The excitation is $96 \mathrm{nrad}$ in each/both planes. The $7 Q_{x}$ and $7 Q_{y}$ resonance are both excited and in addition the 14 th order $7 Q_{x}+7 Q_{y}$. 


\section{Appendix B: Additional figures for fill 6220, random excitation}

\section{Random excitation: Beam losses measured with FBCT}
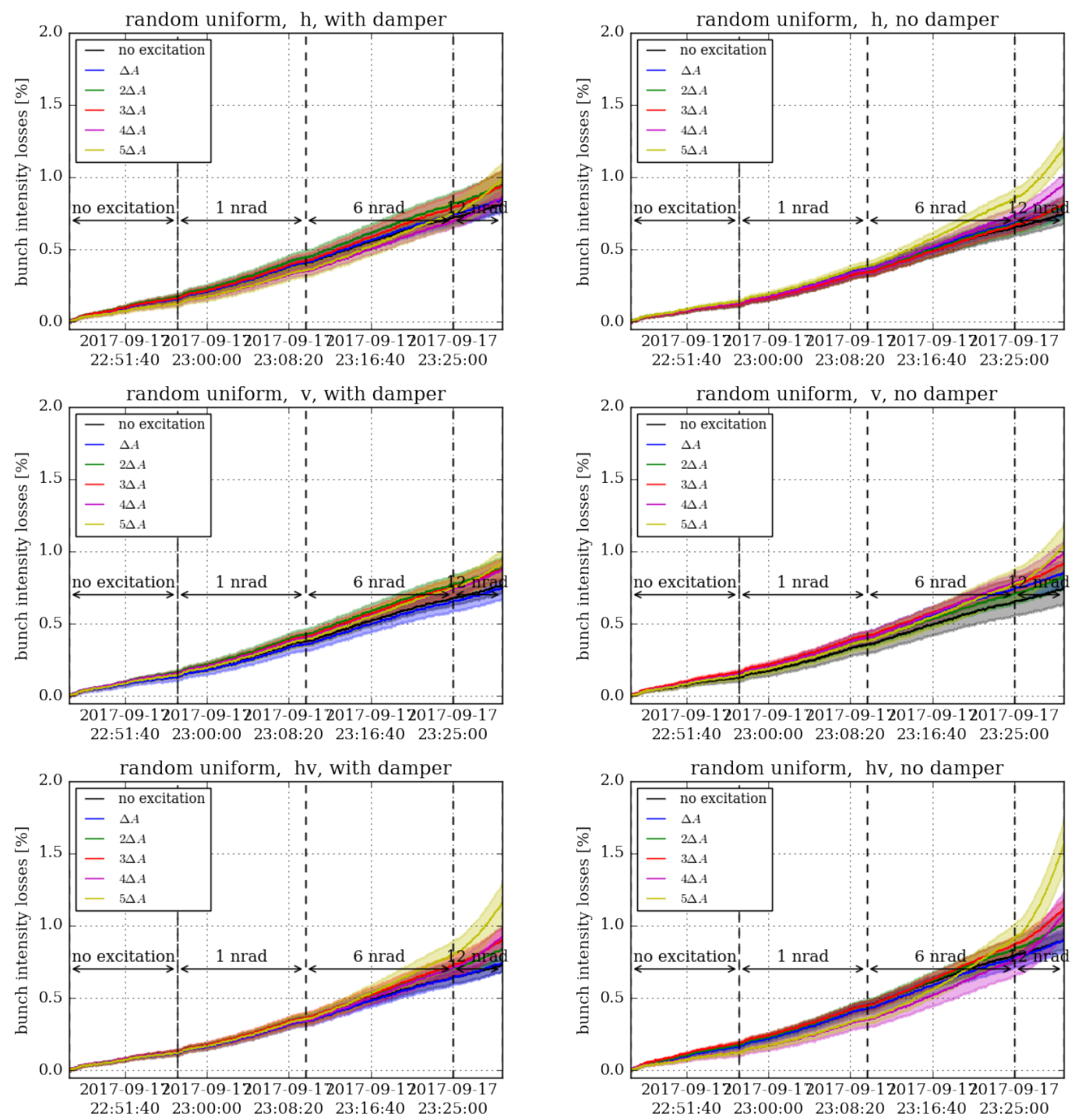

Figure 64. random uniform excitation: Relative bunch intensity losses measured with the FBCTs (Eqn. 3). The average over the group of 6 bunches experiencing the same excitation amplitude is indicated by a solid line together with the $1 \sigma$ standard deviation over the four bunches indicated as an envelope in the same color. The maximum excitation amplitudes are indicated with black arrows. 


\section{Random excitation: BSRT emittance as logged in the LHC Logging database}


Figure 65. random excitation: relative emittance in the horizontal plane measured with the BSRT (Gaussian fit) and as defined in Eqn. 24 together with the $1 \sigma$ standard deviation over the bunches with the same excitation amplitude $n \cdot \Delta A$ indicated as an envelope for each amplitude. For all emittance values a moving average over 10 time stamps is performed. The amplitudes $5 \cdot \Delta A$ and $4 \cdot \Delta A$ overlap for excitation in $\mathrm{H}+\mathrm{V}$. One reason for the behavior could be that the emittance for $4 \cdot \Delta A$ is much smaller than for $5 \cdot \Delta A$ (see Appendix B 2, Fig. 67) 

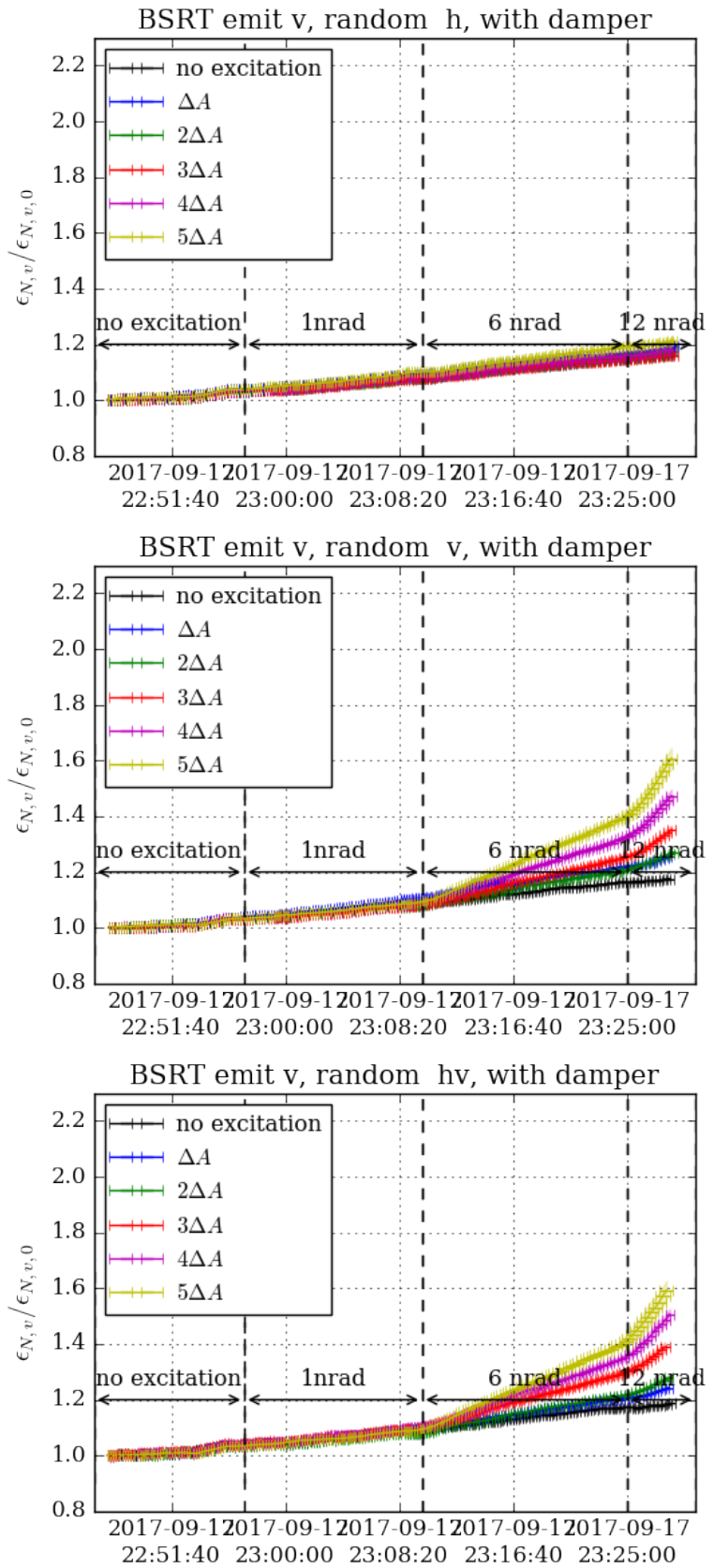
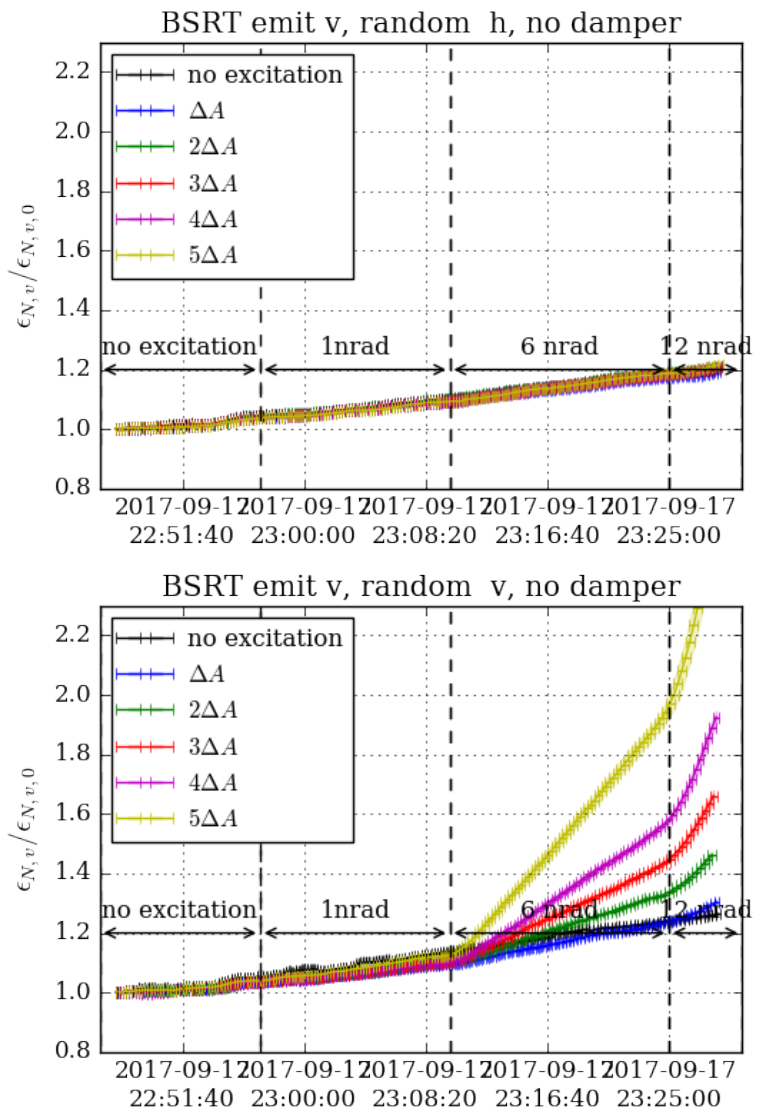

BSRT emit $v$, random hv, no damper

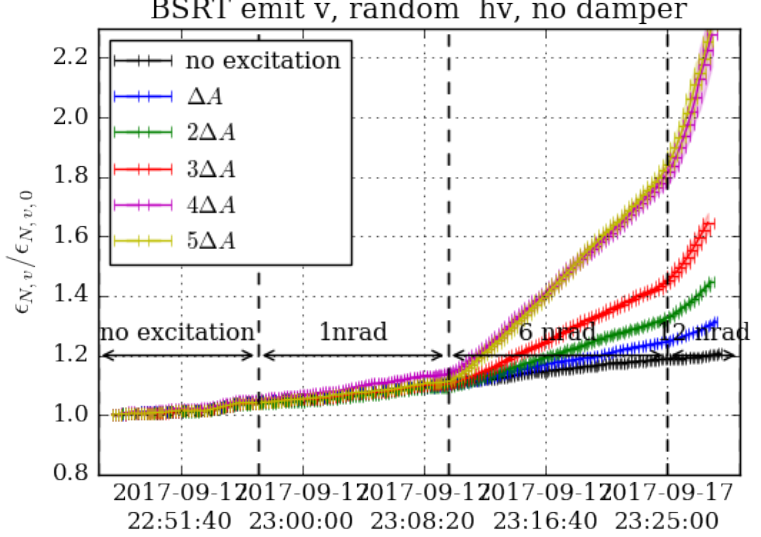

Figure 66. random excitation: relative emittance in the vertical plane measured with the BSRT (Gaussian fit) and as defined in Eqn. 24 together with the $1 \sigma$ standard deviation over the bunches with the same excitation amplitude $n \cdot \Delta A$ indicated as an envelope for each amplitude. For all emittance values a moving average over 10 time stamps is performed. The amplitudes $5 \cdot \Delta A$ and $4 \cdot \Delta A$ overlap for excitation in $\mathrm{H}+\mathrm{V}$. One reason for the behavior could be that the emittance for $4 \cdot \Delta A$ is much smaller than for $5 \cdot \Delta A$ (see Appendix B 2, Fig. 68) 



Figure 67. random excitation: emittance in the horizontal plane measured with the BSRT (Gaussian fit) and as defined in Eqn. 22 together with the $1 \sigma$ standard deviation over the bunches with the same excitation amplitude $n \cdot \Delta A$ indicated as an envelope for each amplitude. For all emittance values a moving average over 10 time stamps is performed. 

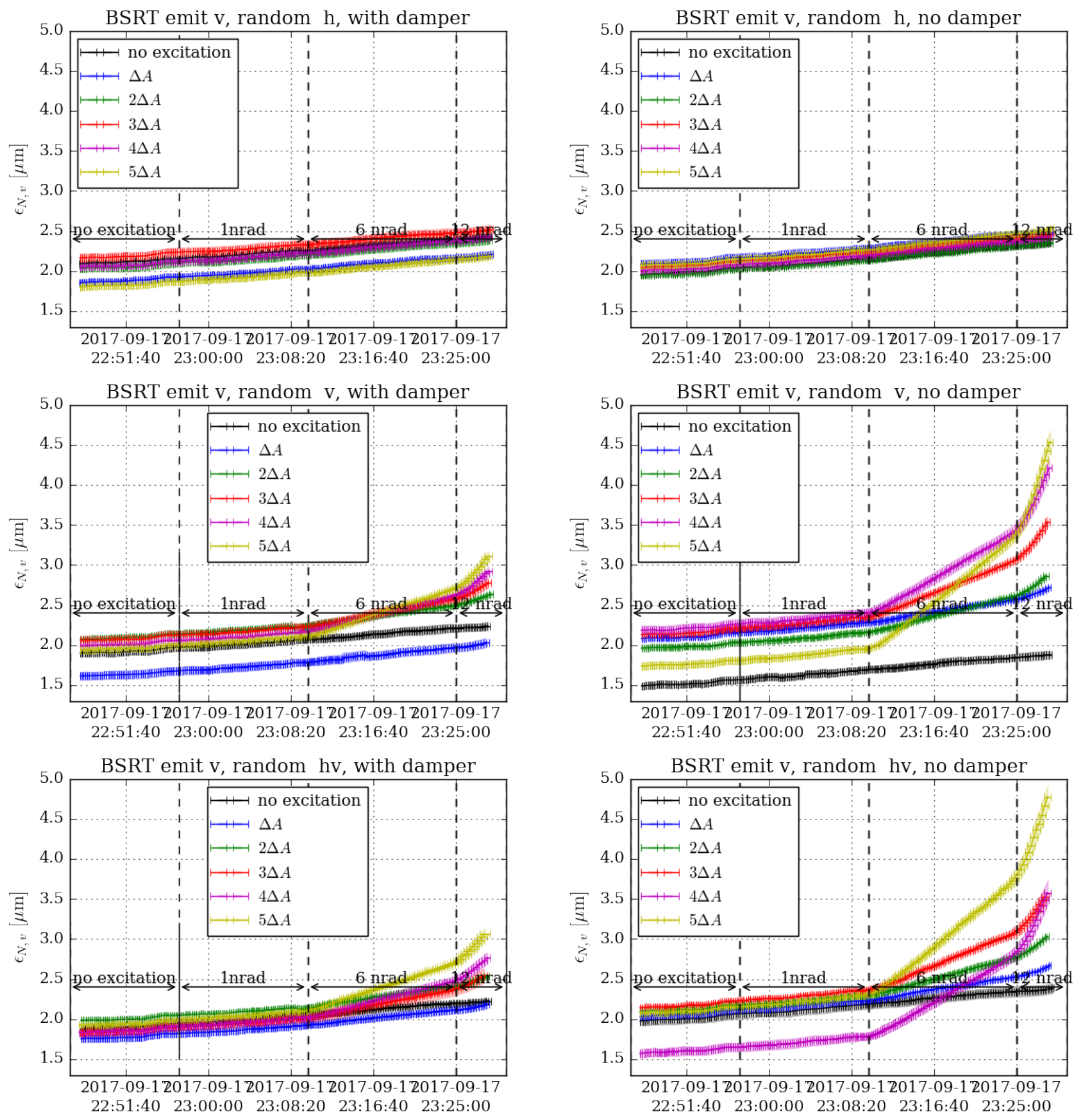

Figure 68. random excitation: relative emittance in the vertical plane measured with the BSRT (Gaussian fit) and as defined in Eqn. 22 together with the $1 \sigma$ standard deviation over the bunches with the same excitation amplitude $n \cdot \Delta A$ indicated as an envelope for each amplitude. For all emittance values a moving average over 10 time stamps is performed. 

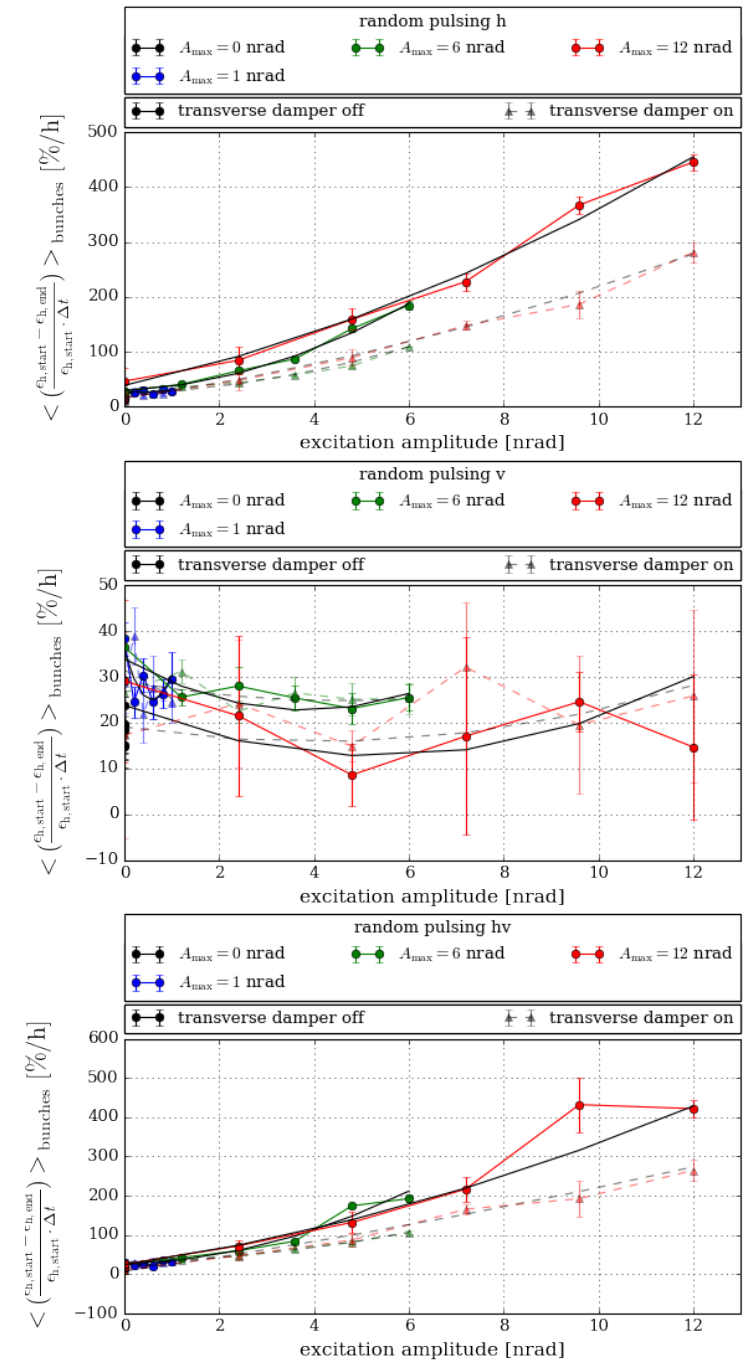

Figure 69. random excitation: Average emittance growth rate in the horizontal (left) and vertical (right) plane measured with the BSRT and as defined in Eqn. 25 using the emittance $\varepsilon$ from the LHC Logging database. The measurements for the different maximum excitation amplitudes $A_{\max }$ are indicated in different colors. The bunches for which the transverse damper is active are shown with solid dots and the bunches for which the transverse damper is not active are shown with slightly transparent triangles. The emittance growth rate increases approximately quadratically with the excitation amplitude $n \cdot \Delta A$, if a dependence on the excitation amplitude is observed. The quadratic fit to the emittance growth rate is indicated with a solid line for the bunches with transverse damper active and with a dashed line for the bunches with the transverse damper not active. For all emittance values a moving average over 10 time stamps is performed.
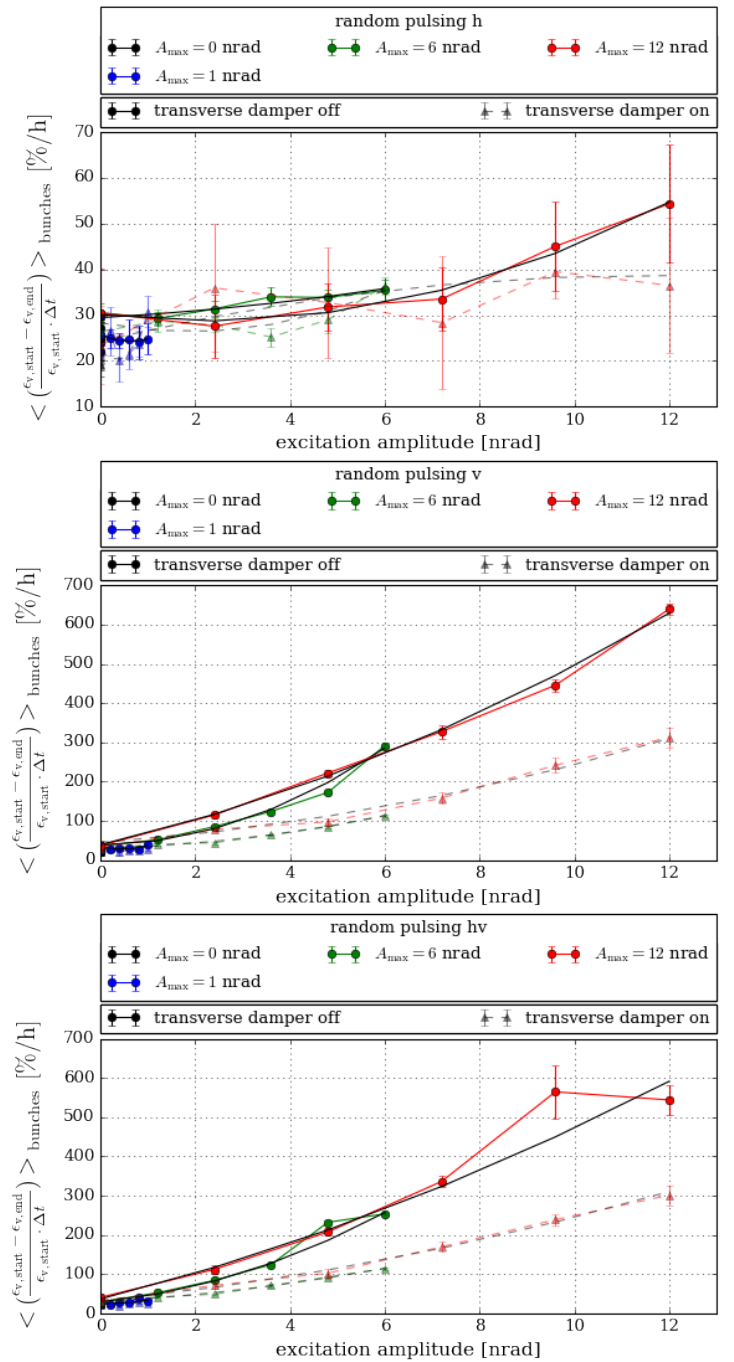


\section{Random excitation: BSRT profile analysis}

\section{a. Random excitation: BSRT profiles - statistical parameters}
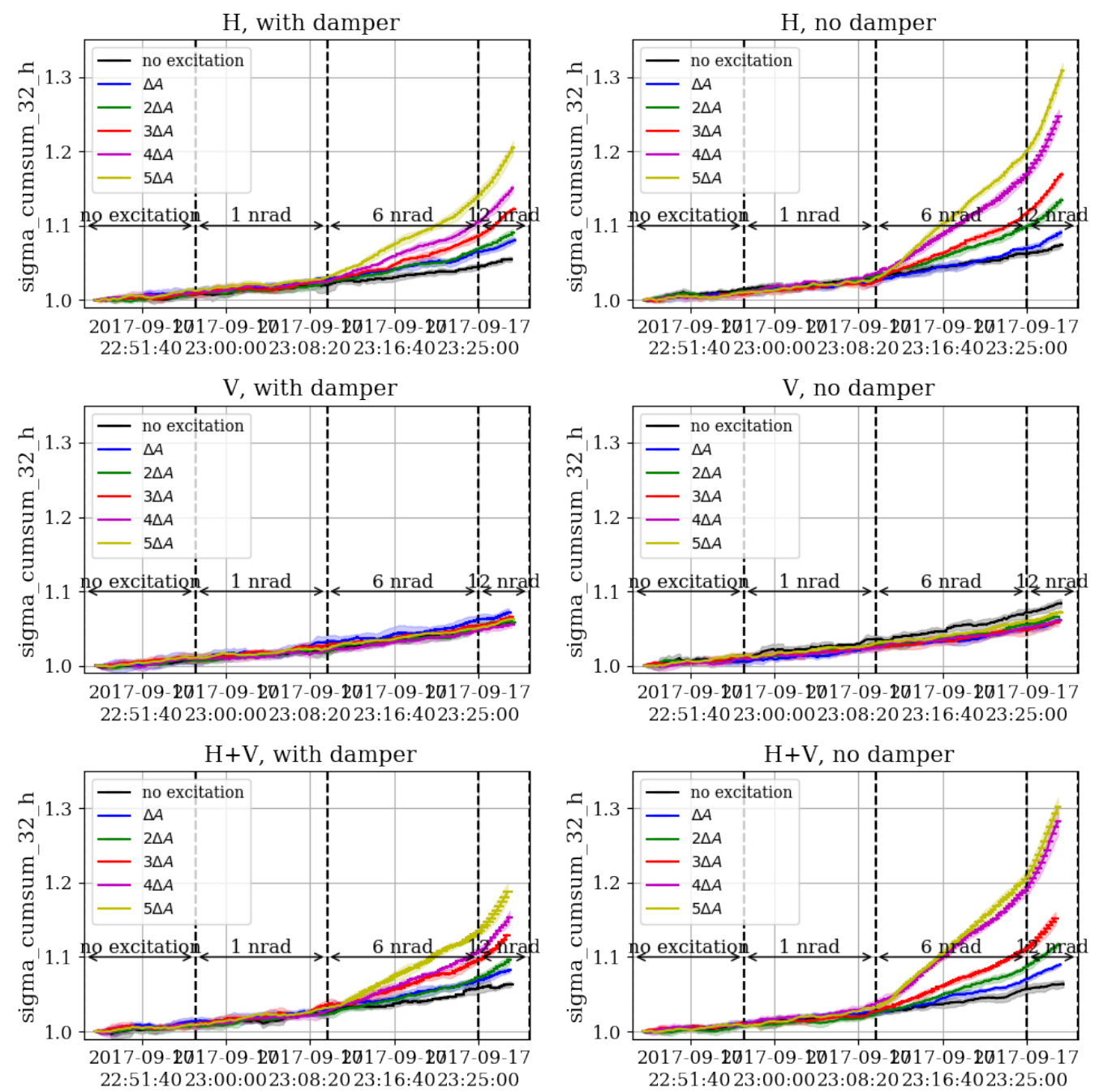

Figure 70. random excitation: relative beam sigma calculated over the cumulative sum (Eqn. 17) in the horizontal plane measured with the BSRT together with the $1 \sigma$ standard deviation over the bunches with the same excitation amplitude $n \cdot \Delta A$ indicated as an envelope for each amplitude. For all beam sigma values a moving average over 10 time stamps is performed and the average, explicitly not moving average, profiles are used. 
$\mathrm{H}$, with damper
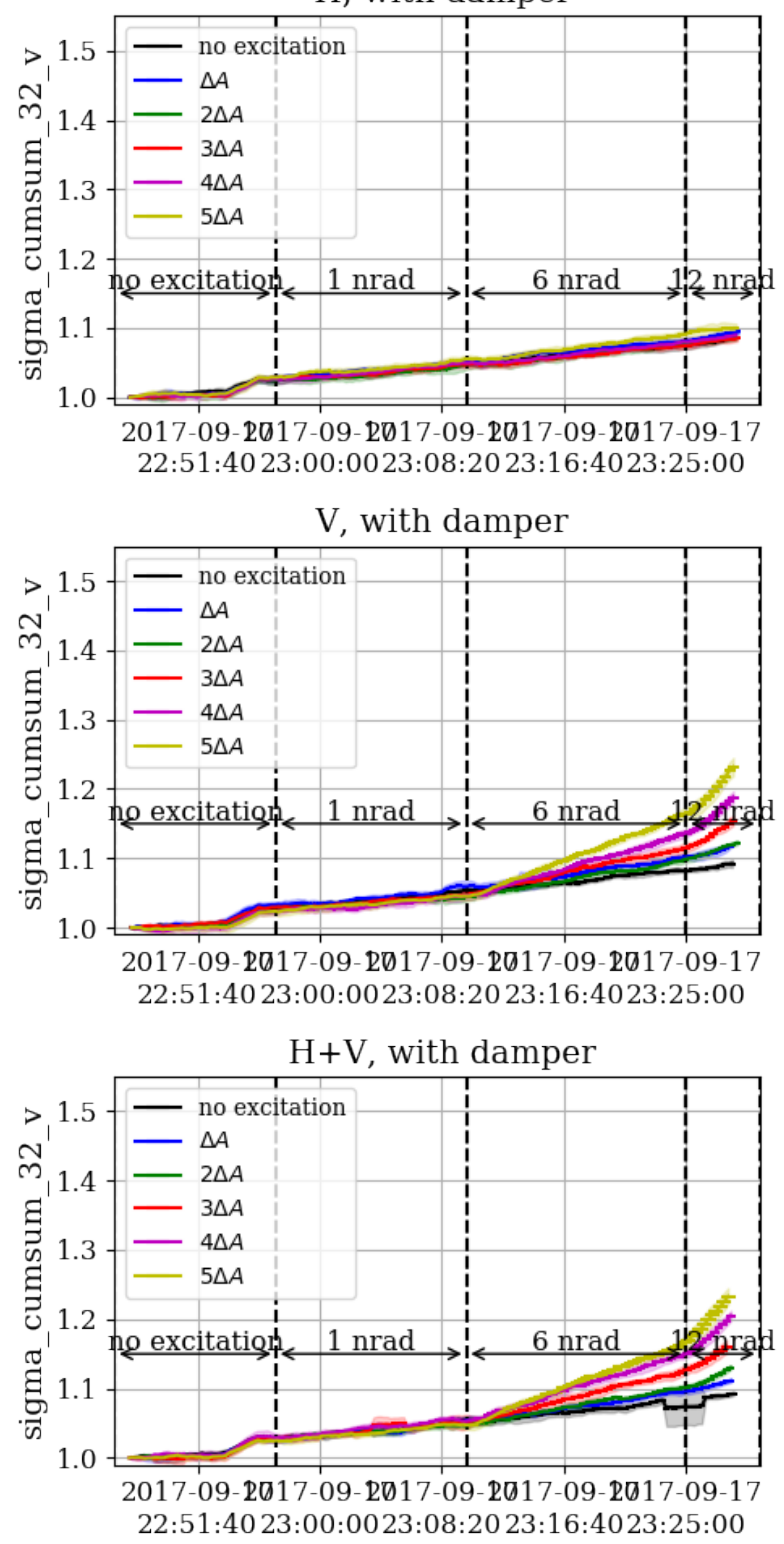

H, no damper
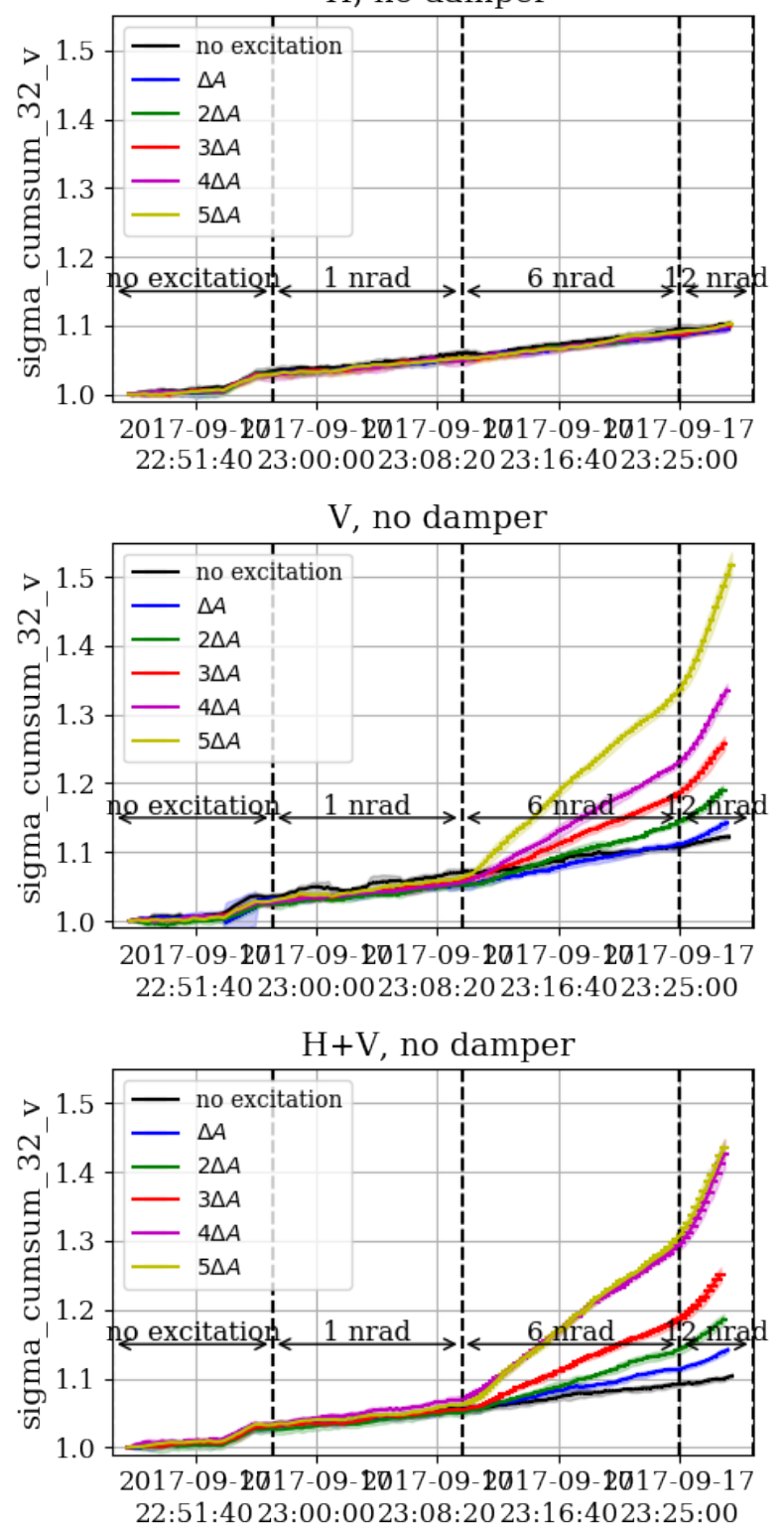

Figure 71. random excitation: relative beam sigma calculated over the cumulative sum (Eqn. 17) in the vertical plane measured with the BSRT together with the $1 \sigma$ standard deviation over the bunches with the same excitation amplitude $n \cdot \Delta A$ indicated as an envelope for each amplitude. For all beam sigma values a moving average over 10 time stamps is performed and the average, explicitly not moving average, profiles are used. The increase of the beam sigma in the vertical plane between 22:53 and 22:55 is due to the orbit shift shown in Appendix B 3 a, Figs. 72-73. 
$H$, with damper

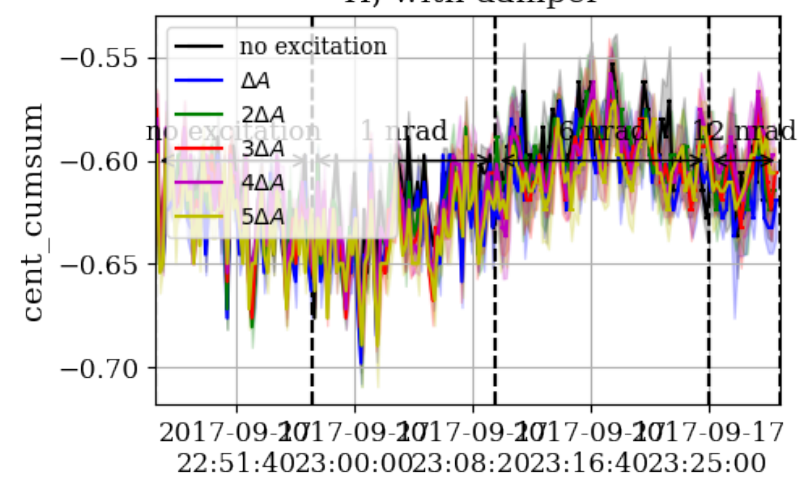

$\mathrm{V}$, with damper
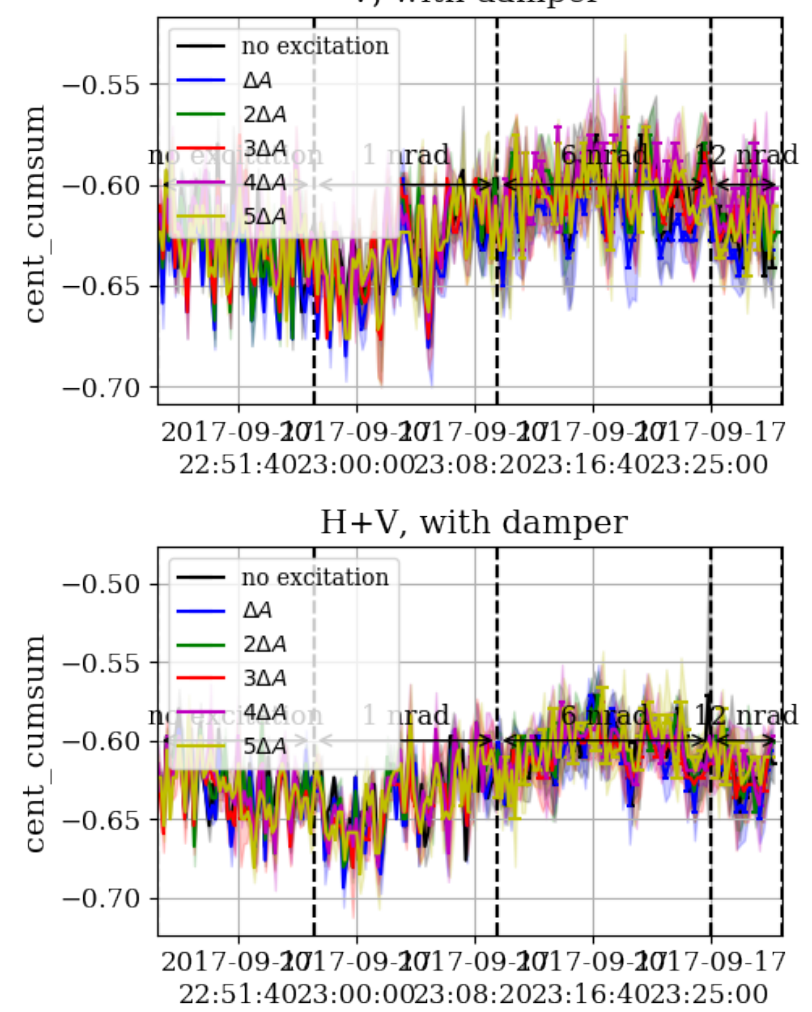
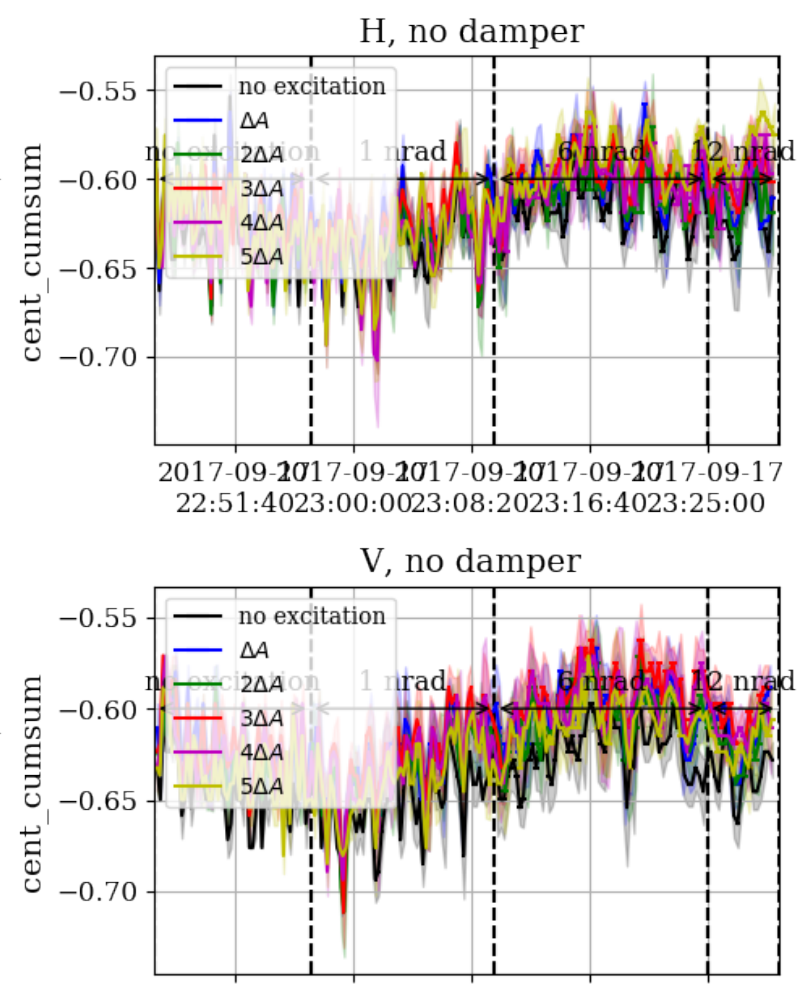

2017-09-2017-09-2017-09-2017-09-2017-09-17 22:51:4023:00:0023:08:2023:16:4023:25:00

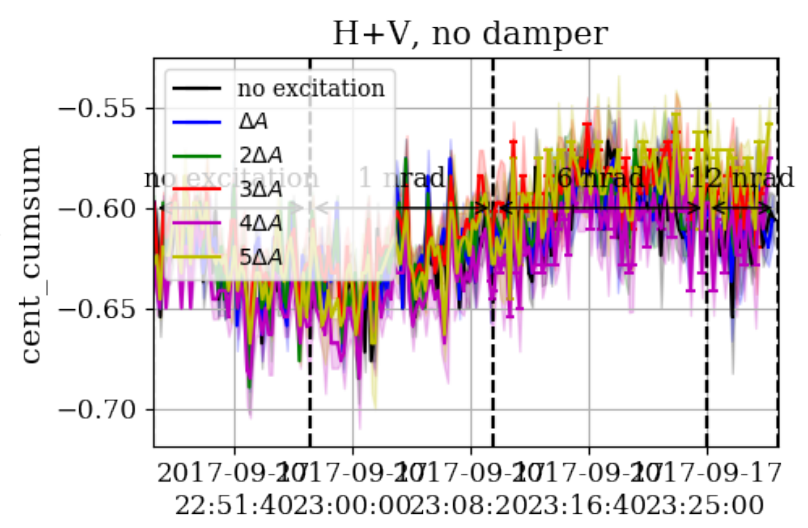

Figure 72. random excitation: centroid calculated over the cumulative sum (Eqn. 17) in the horizontal plane measured with the BSRT together with the $1 \sigma$ standard deviation over the bunches with the same excitation amplitude $n \cdot \Delta A$ indicated as an envelope for each amplitude. No moving average over the profiles nor parameter is taken in order to show the un-smoothed oscillations. 
$\mathrm{H}$, with damper

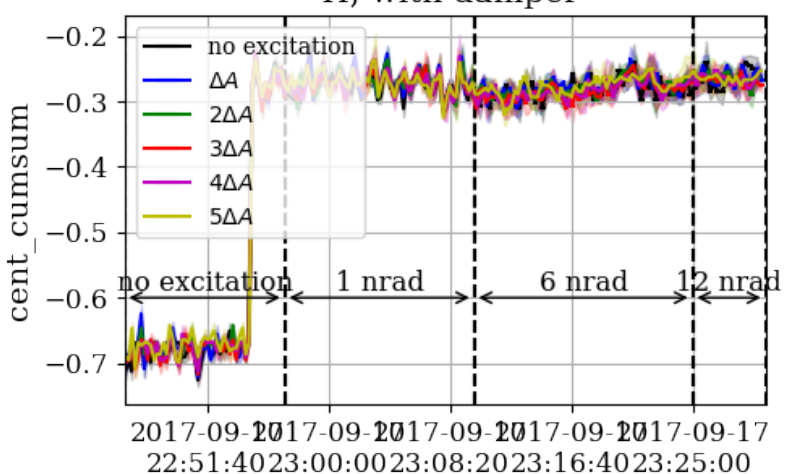

$\mathrm{V}$, with damper

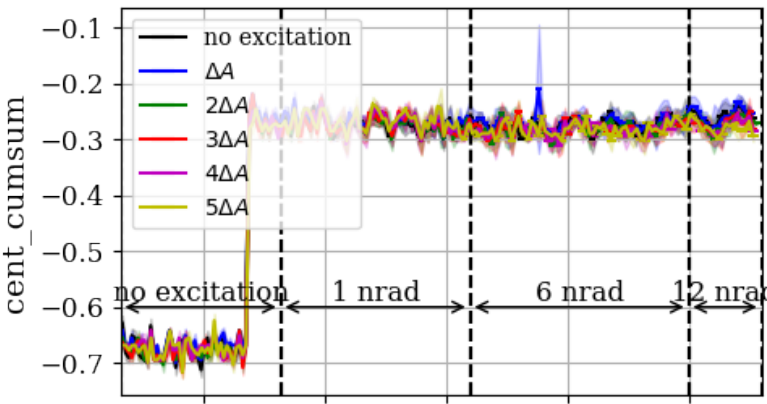

2017-09-2017-09-2017-09-2017-09-2017-09-17 22:51:4023:00:0023:08:2023:16:4023:25:00

$\mathrm{H}+\mathrm{V}$, with damper

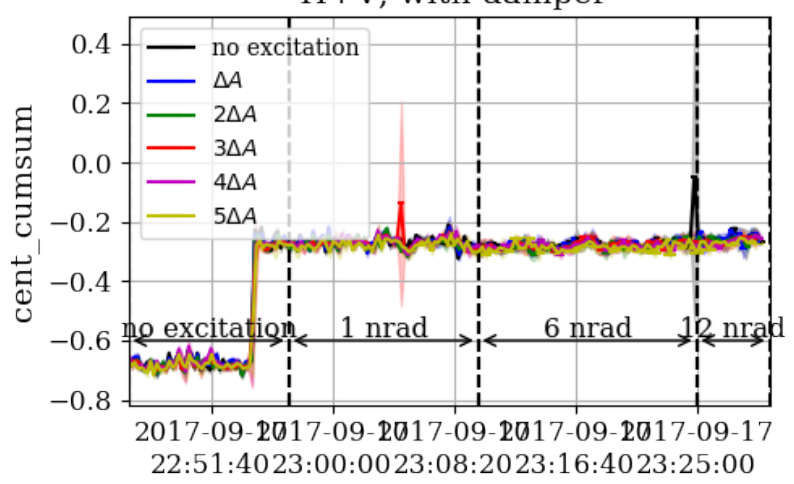

H, no damper


2017-09-2017-09-2017-09-2017-09-2017-09-17 22:51:4023:00:0023:08:2023:16:4023:25:00

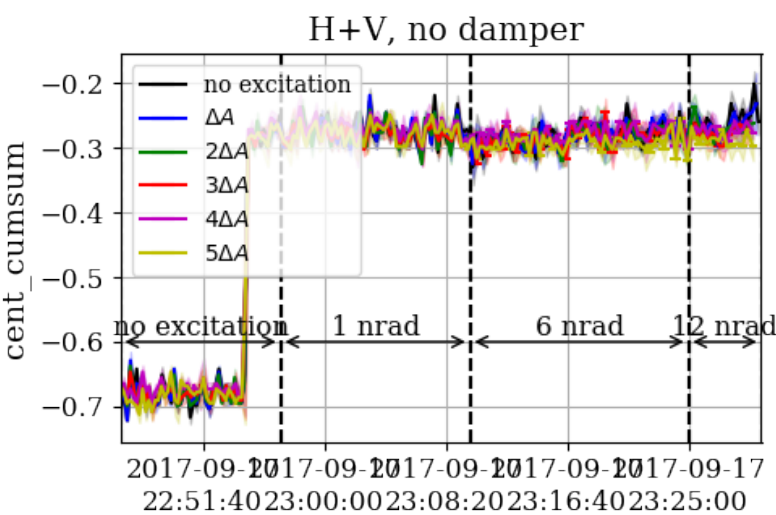

Figure 73. random excitation: centroid calculated over the cumulative sum (Eqn. 17) in the vertical plane measured with the BSRT together with the $1 \sigma$ standard deviation over the bunches with the same excitation amplitude $n \cdot \Delta A$ indicated as an envelope for each amplitude. No moving average over the profiles nor parameter is taken in order to show the un-smoothed oscillations. 
b. Random excitation: BSRT profiles - q-Gaussian fit parameters in the horizontal plane
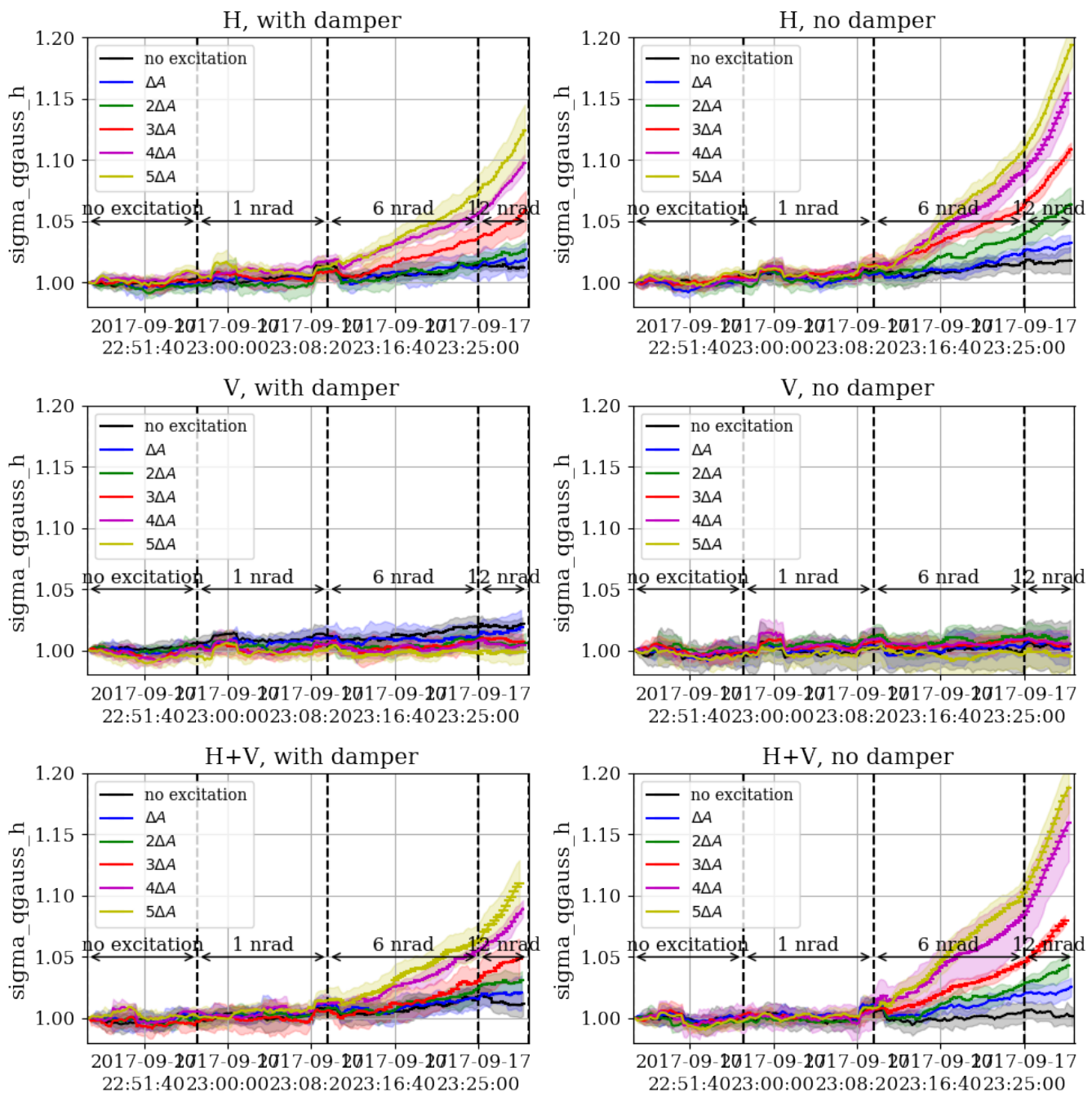

Figure 74. random excitation: relative beam sigma from q-Gaussian fit of the BSRT profiles (Eqn. 9) in the horizontal plane measured with the BSRT together with the $1 \sigma$ standard deviation over the bunches with the same excitation amplitude $n \cdot \Delta A$ indicated as an envelope for each amplitude. For all values the moving average profiles are used without any further averaging afterwards. 
$\mathrm{H}$, with damper


22:51:40 23:00:0023:08:20 23:16:4023:25:00






Figure 75. random excitation: relative fit parameter $q$ from q-Gaussian fit of the BSRT profiles (Eqn. 9) in the horizontal plane measured with the BSRT together with the $1 \sigma$ standard deviation over the bunches with the same excitation amplitude $n \cdot \Delta A$ indicated as an envelope for each amplitude. For all values the moving average profiles are used without any further averaging afterwards. 

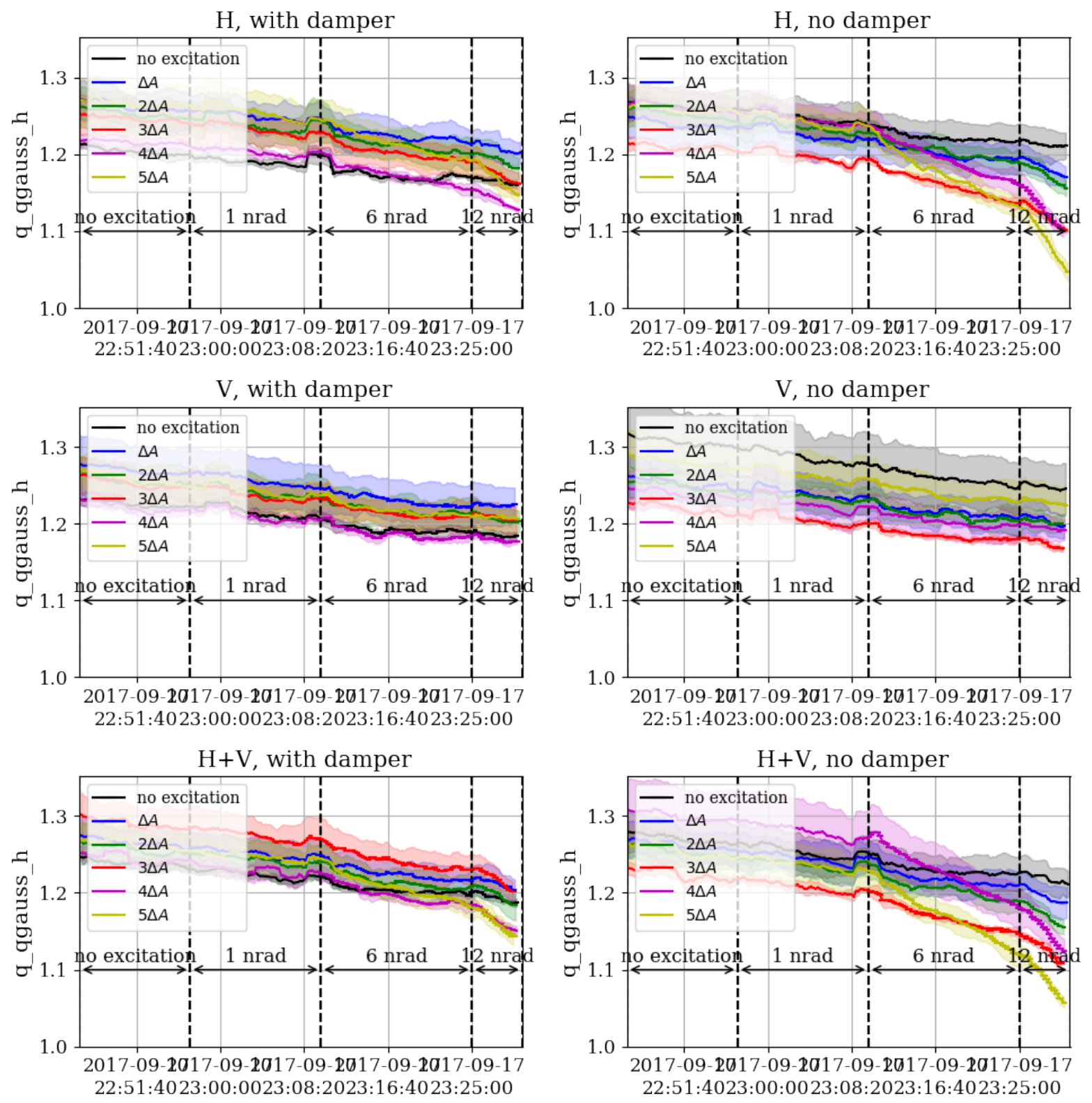

Figure 76. random excitation: fit parameter $q$ from q-Gaussian fit of the BSRT profiles (Eqn. 9) in the horizontal plane measured with the BSRT together with the $1 \sigma$ standard deviation over the bunches with the same excitation amplitude $n \cdot \Delta A$ indicated as an envelope for each amplitude. For all values the moving average profiles are used without any further averaging afterwards. 
$\mathrm{H}$, with damper
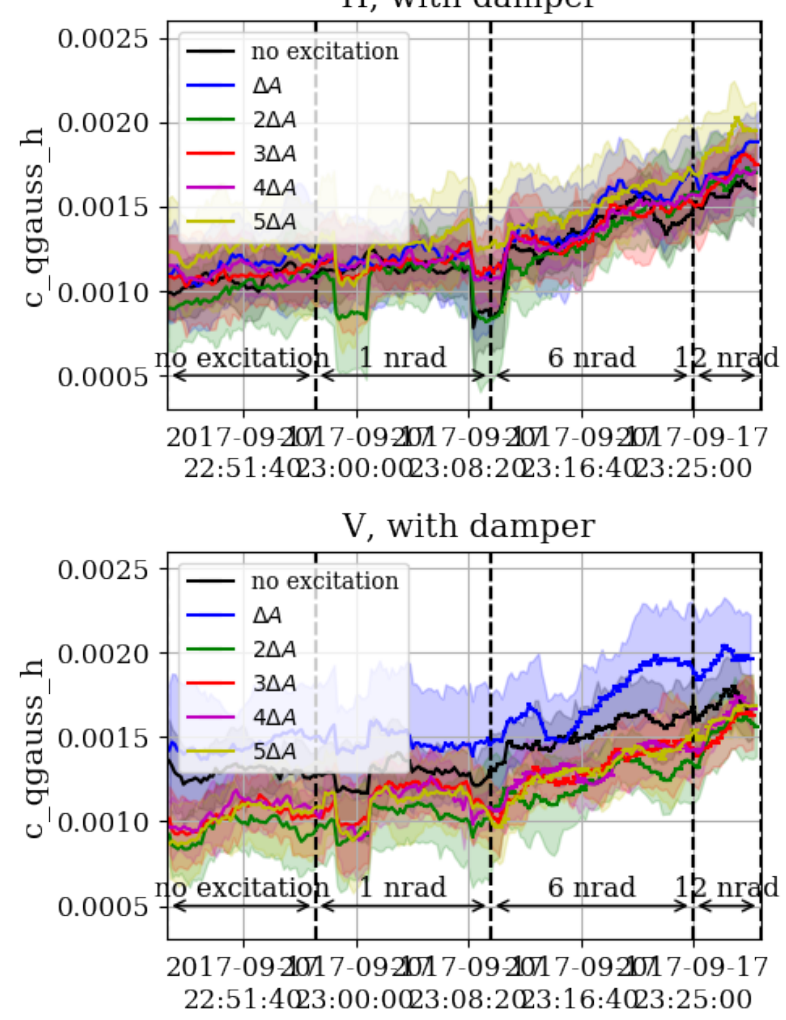

$\mathrm{H}+\mathrm{V}$, with damper



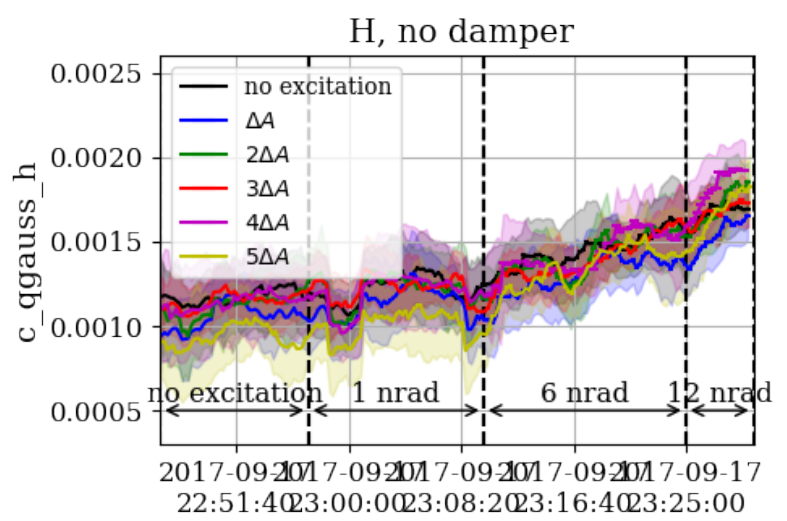


Figure 77. random excitation: fit parameter $c$ from q-Gaussian fit of the BSRT profiles (Eqn. 9) in the horizontal plane measured with the BSRT together with the $1 \sigma$ standard deviation over the bunches with the same excitation amplitude $n \cdot \Delta A$ indicated as an envelope for each amplitude. For all values the moving average profiles are used without any further averaging afterwards. 

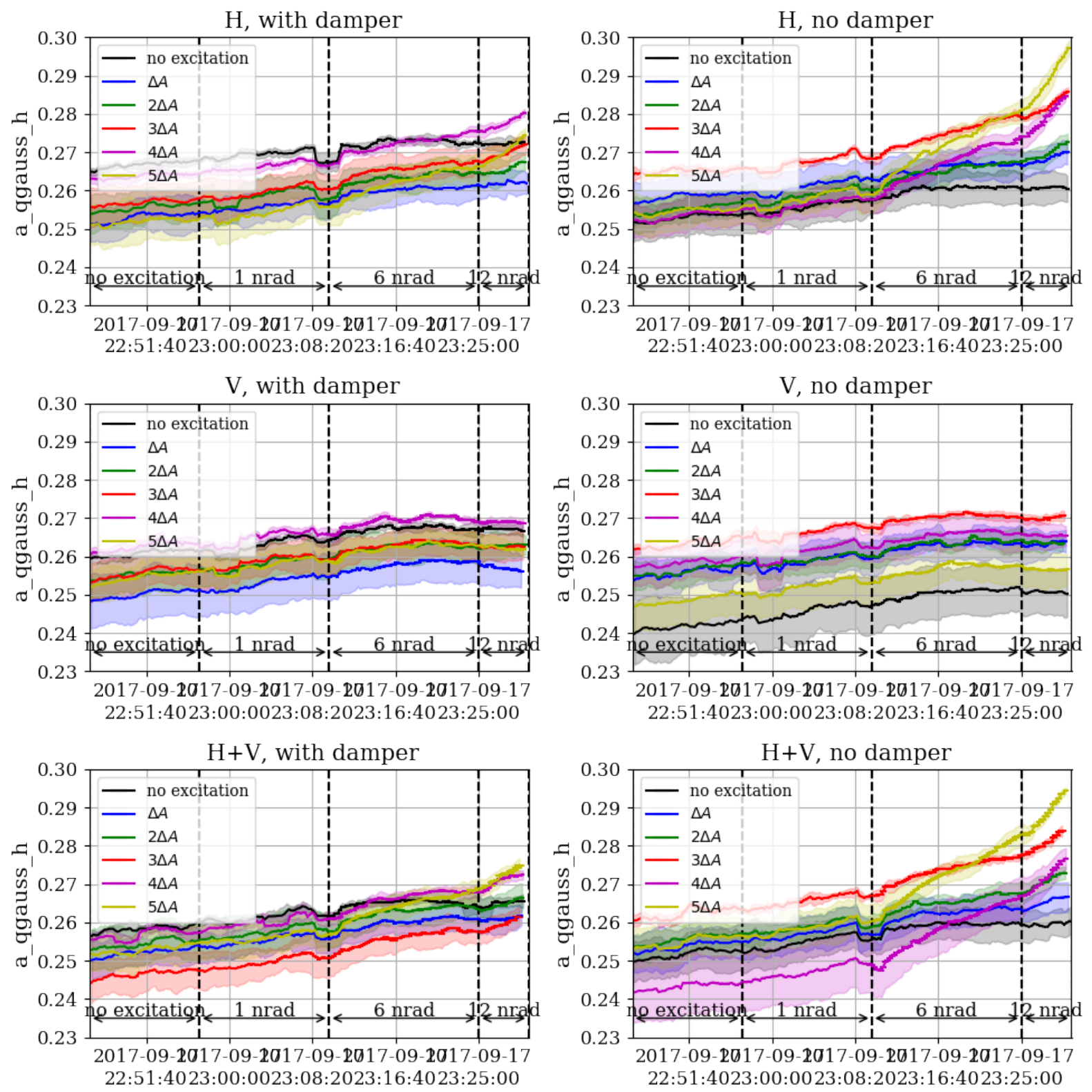

Figure 78. random excitation: fit parameter $a$ from q-Gaussian fit of the BSRT profiles (Eqn. 9) in the horizontal plane measured with the BSRT together with the $1 \sigma$ standard deviation over the bunches with the same excitation amplitude $n \cdot \Delta A$ indicated as an envelope for each amplitude. For all values the moving average profiles are used without any further averaging afterwards. 

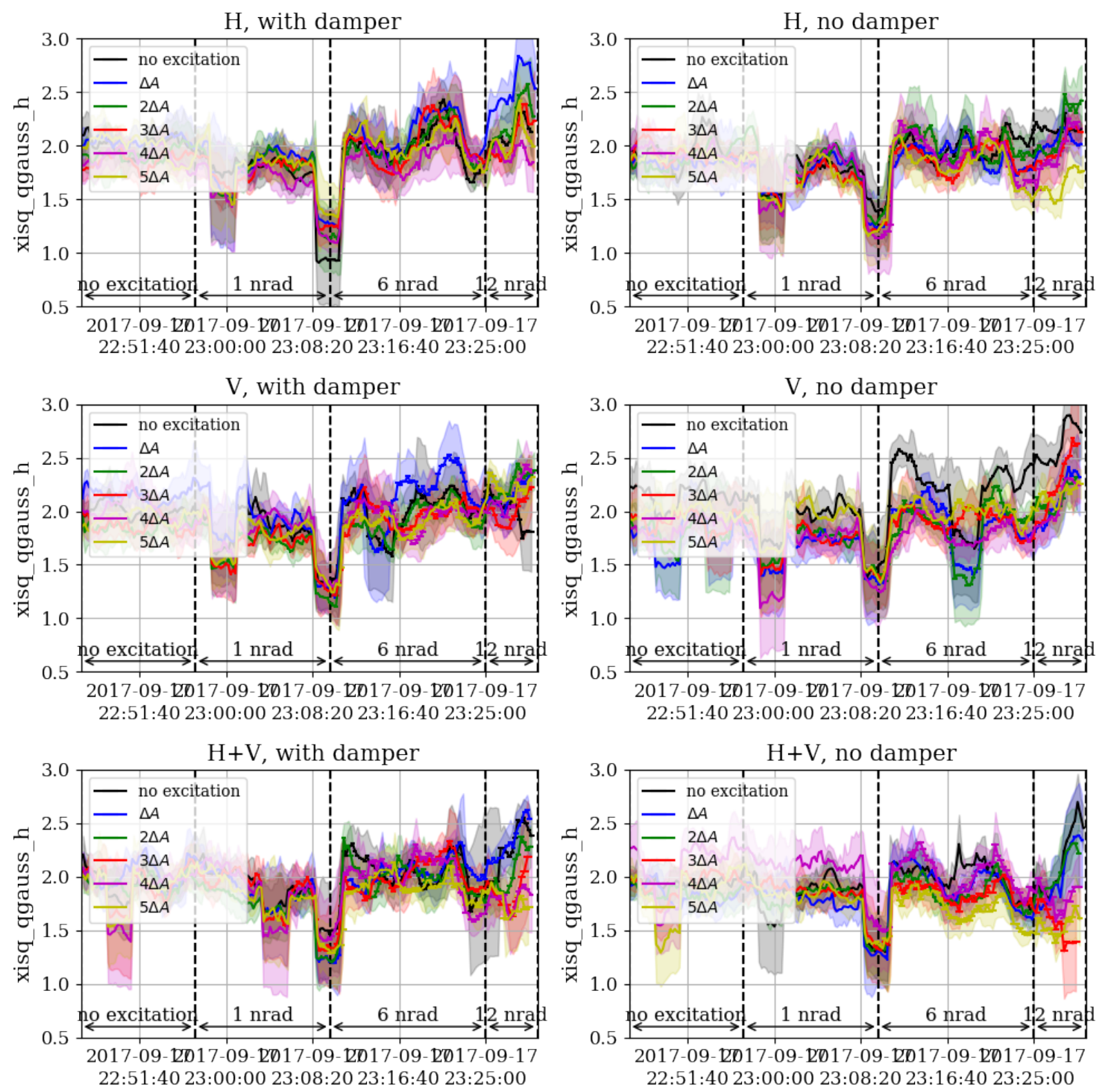

Figure 79. random excitation: normalized $\chi^{2}$ of q-Gaussian fit of the BSRT profiles (Eqn. 9) in the horizontal plane measured with the BSRT together with the $1 \sigma$ standard deviation over the bunches with the same excitation amplitude $n \cdot \Delta A$ indicated as an envelope for each amplitude. For all values the moving average profiles are used without any further averaging afterwards. 

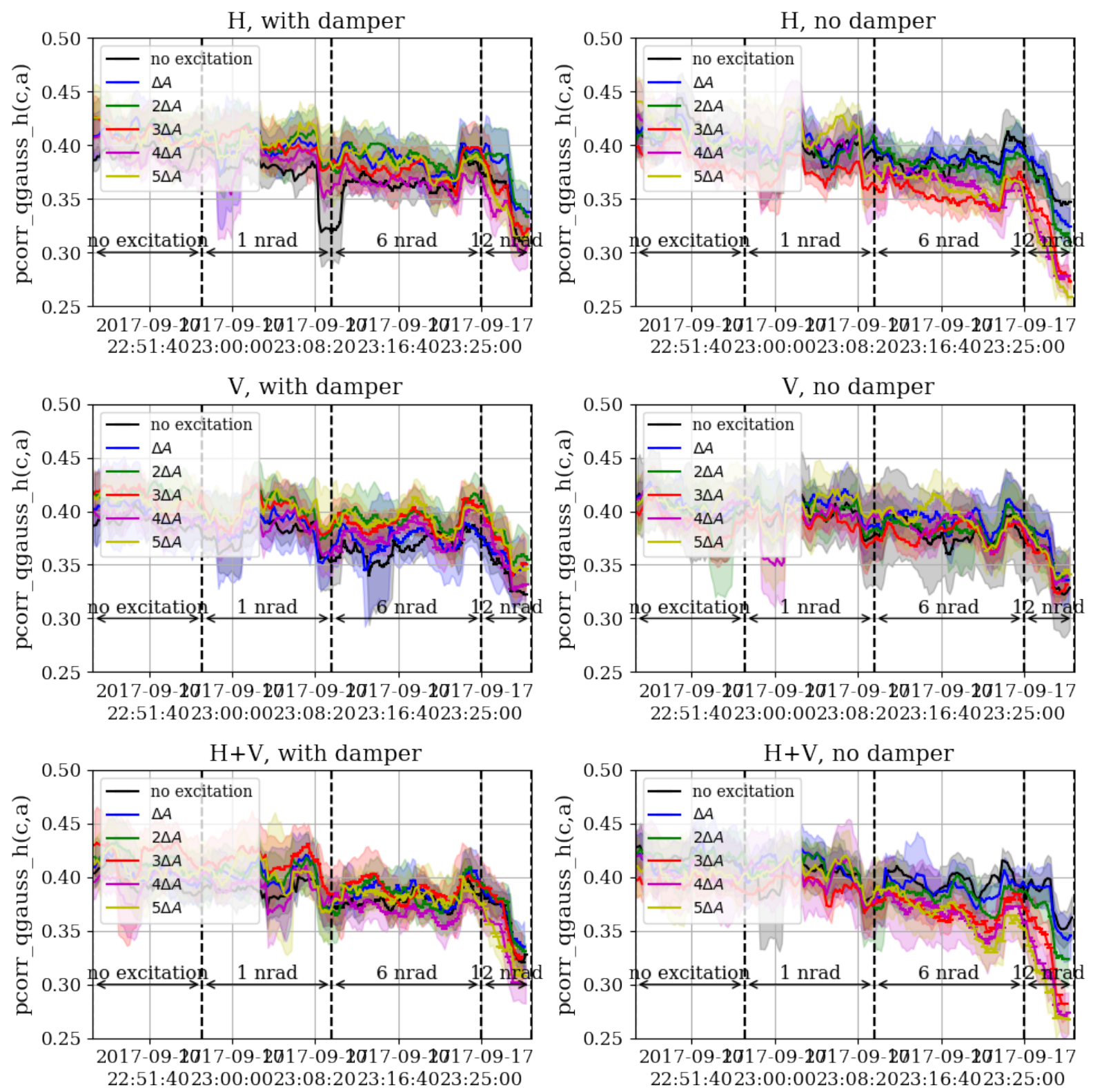

Figure 80. random excitation: correlation coefficient between fit parameter $c$ and $a$ from q-Gaussian fit of the BSRT profiles (Eqn. 9) in the horizontal plane measured with the BSRT together with the $1 \sigma$ standard deviation over the bunches with the same excitation amplitude $n \cdot \Delta A$ indicated as an envelope for each amplitude. For all values the moving average profiles are used without any further averaging afterwards. 

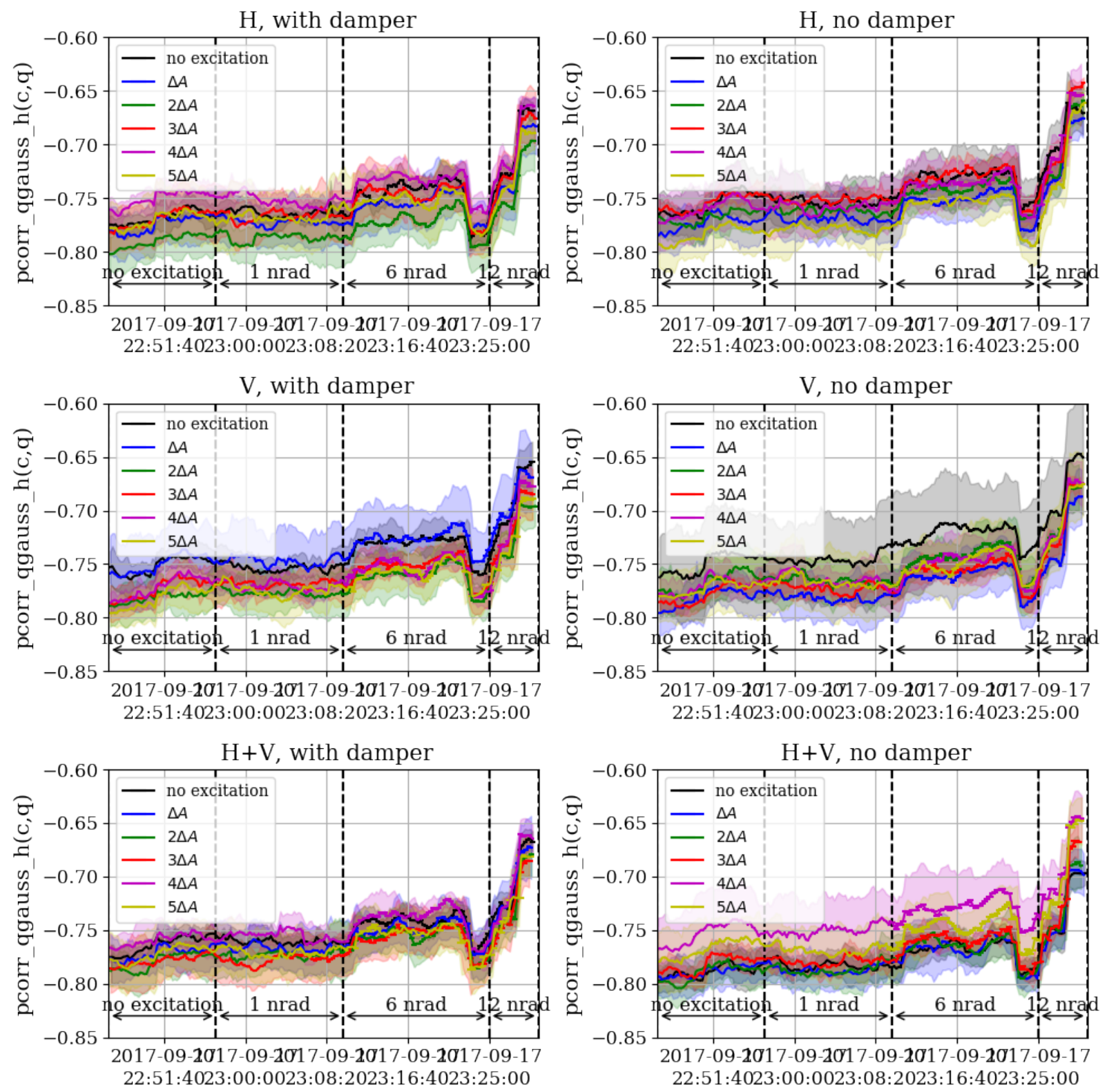

Figure 81. random excitation: correlation coefficient between fit parameter $c$ and $q$ from q-Gaussian fit of the BSRT profiles (Eqn. 9) in the horizontal plane measured with the BSRT together with the $1 \sigma$ standard deviation over the bunches with the same excitation amplitude $n \cdot \Delta A$ indicated as an envelope for each amplitude. For all values the moving average profiles are used without any further averaging afterwards. 

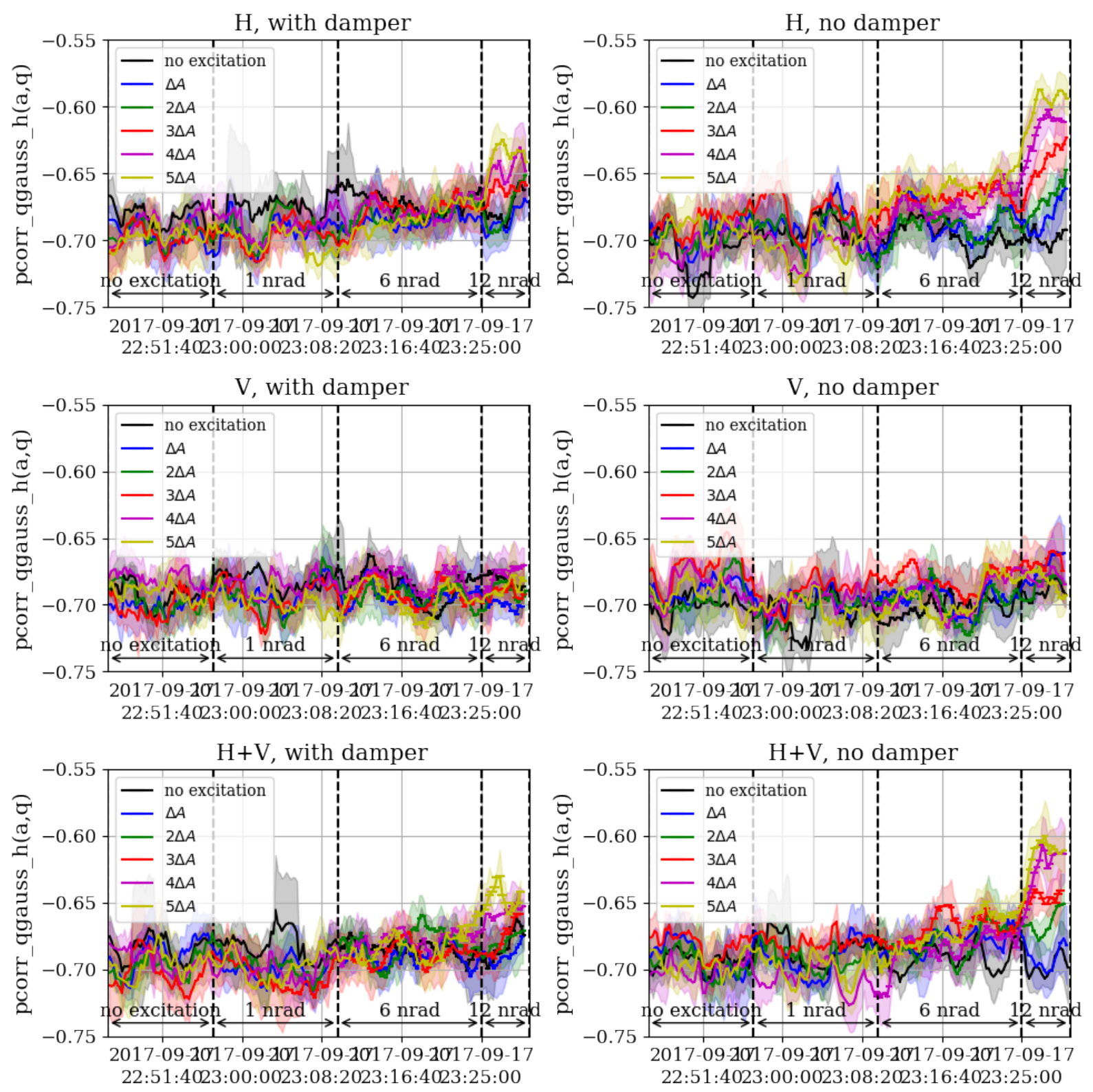

Figure 82. random excitation: correlation coefficient between fit parameter $a$ and $q$ from q-Gaussian fit of the BSRT profiles (Eqn. 9) in the horizontal plane measured with the BSRT together with the $1 \sigma$ standard deviation over the bunches with the same excitation amplitude $n \cdot \Delta A$ indicated as an envelope for each amplitude. For all values the moving average profiles are used without any further averaging afterwards. 
c. Random excitation: BSRT profiles - q-Gaussian fit parameters in the vertical plane
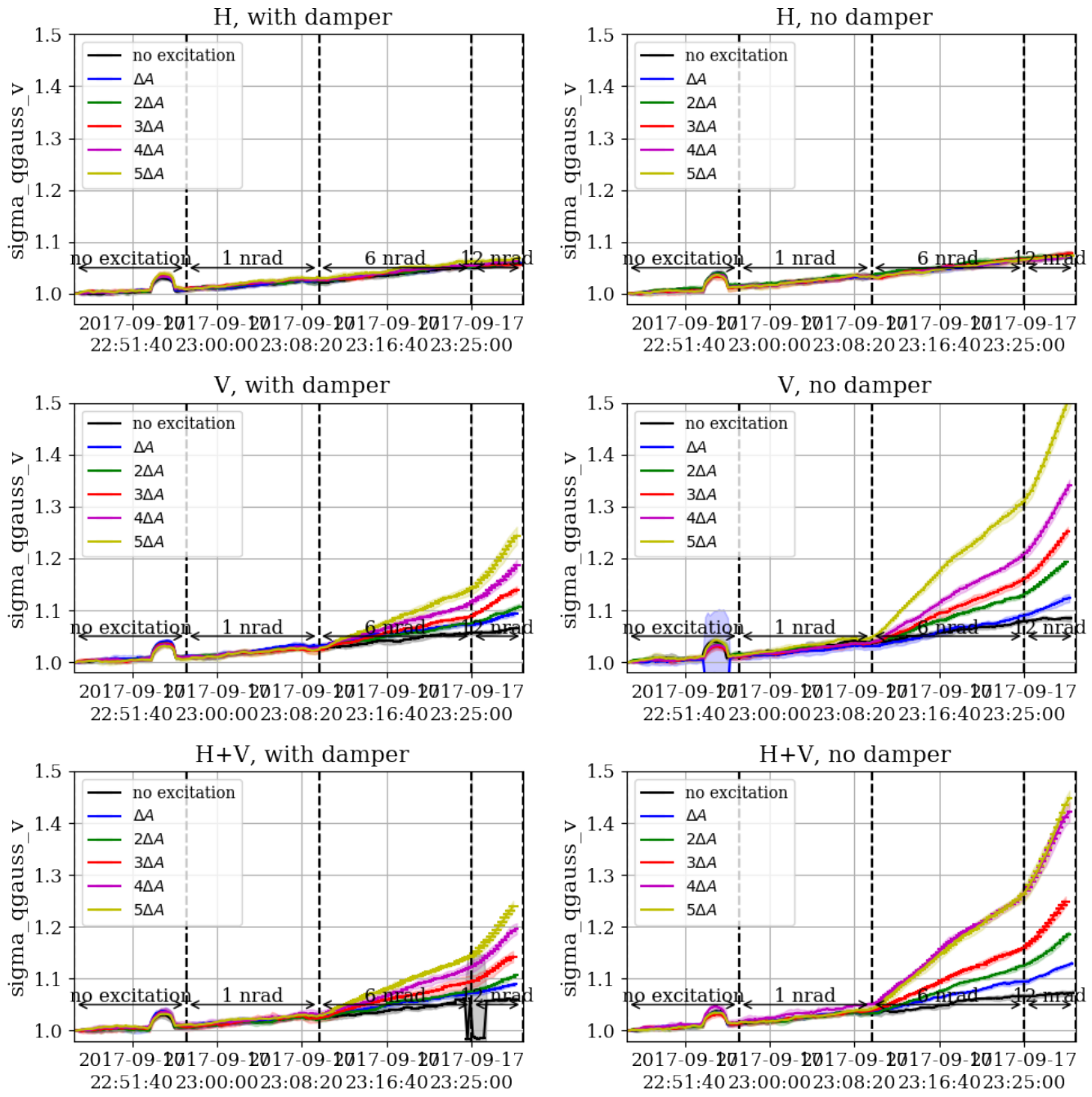

Figure 83. random excitation: relative beam sigma from q-Gaussian fit of the BSRT profiles (Eqn. 9) in the vertical plane measured with the BSRT together with the $1 \sigma$ standard deviation over the bunches with the same excitation amplitude $n \cdot \Delta A$ indicated as an envelope for each amplitude. For all values the moving average profiles are used without any further averaging afterwards. The increase of the beam sigma between 22:53 and 22:55 is due to the orbit shift shown in Appendix B 3 a, Figs. 72-73. 

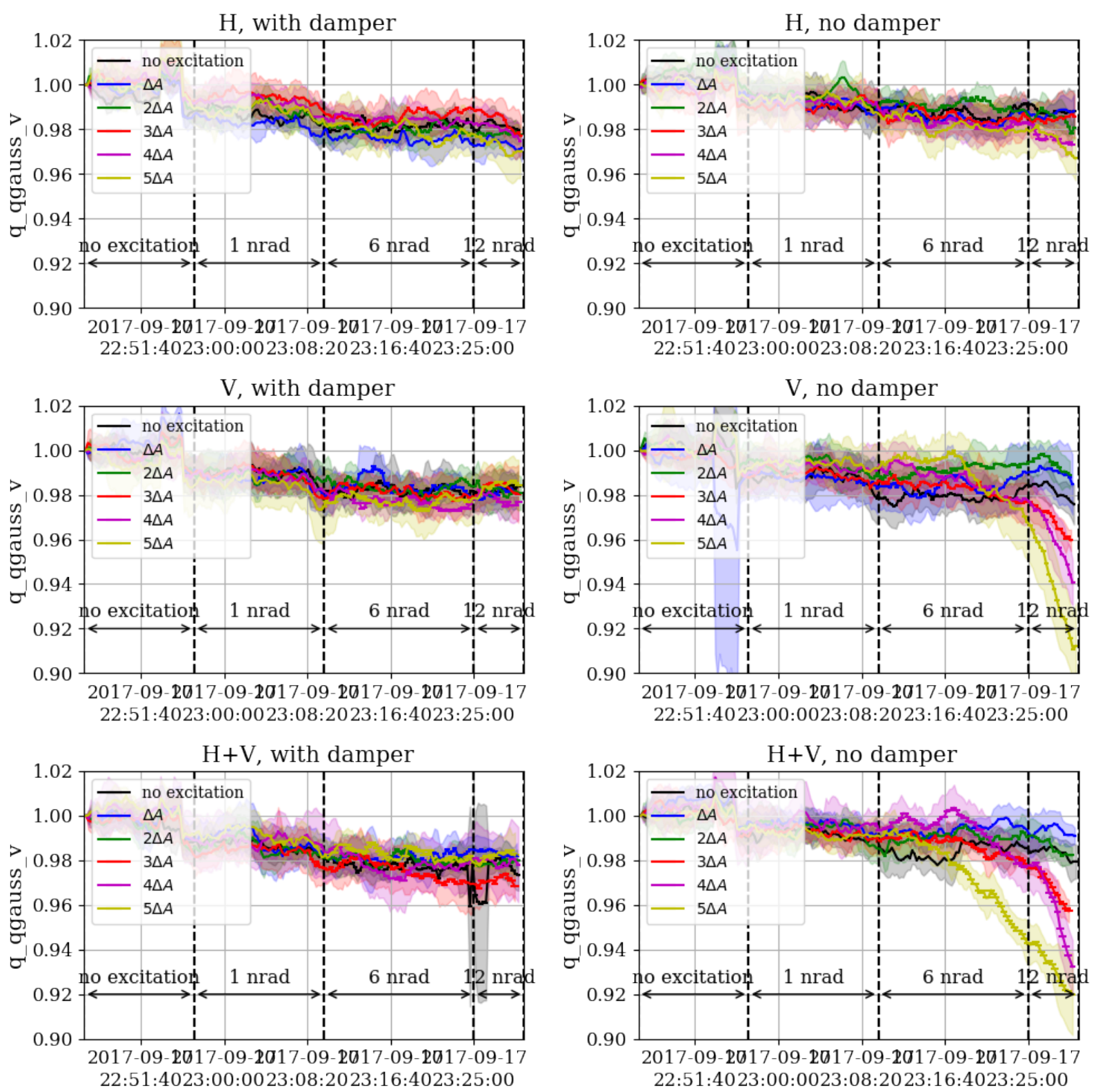

Figure 84. random excitation: relative fit parameter $q$ from q-Gaussian fit of the BSRT profiles (Eqn. 9) in the vertical plane measured with the BSRT together with the $1 \sigma$ standard deviation over the bunches with the same excitation amplitude $n \cdot \Delta A$ indicated as an envelope for each amplitude. For all values the moving average profiles are used without any further averaging afterwards. The change of the relative fit parameter $q$ between 22:53 and 22:55 is due to the orbit shift shown in Appendix B 3 a, Figs. 72-73. 



Figure 85. random excitation: fit parameter $q$ from q-Gaussian fit of the BSRT profiles (Eqn. 9) in the vertical plane measured with the BSRT together with the $1 \sigma$ standard deviation over the bunches with the same excitation amplitude $n \cdot \Delta A$ indicated as an envelope for each amplitude. For all values the moving average profiles are used without any further averaging afterwards. The change of the fit parameter $q$ between 22:53 and 22:55 is due to the orbit shift shown in Appendix B 3 a, Figs. 72-73. 



Figure 86. random excitation: fit parameter $c$ from q-Gaussian fit of the BSRT profiles (Eqn. 9) in the vertical plane measured with the BSRT together with the $1 \sigma$ standard deviation over the bunches with the same excitation amplitude $n \cdot \Delta A$ indicated as an envelope for each amplitude. For all values the moving average profiles are used without any further averaging afterwards. The change of the relative fit parameter $c$ between 22:53 and 22:55 is due to the orbit shift shown in Appendix B 3 a, Figs. 72-73. 



Figure 87. random excitation: fit parameter $a$ from q-Gaussian fit of the BSRT profiles (Eqn. 9) in the vertical plane measured with the BSRT together with the $1 \sigma$ standard deviation over the bunches with the same excitation amplitude $n \cdot \Delta A$ indicated as an envelope for each amplitude. For all values the moving average profiles are used without any further averaging afterwards. The change of the relative fit parameter $a$ between 22:53 and 22:55 is due to the orbit shift shown in Appendix B 3 a, Figs. 72-73. 

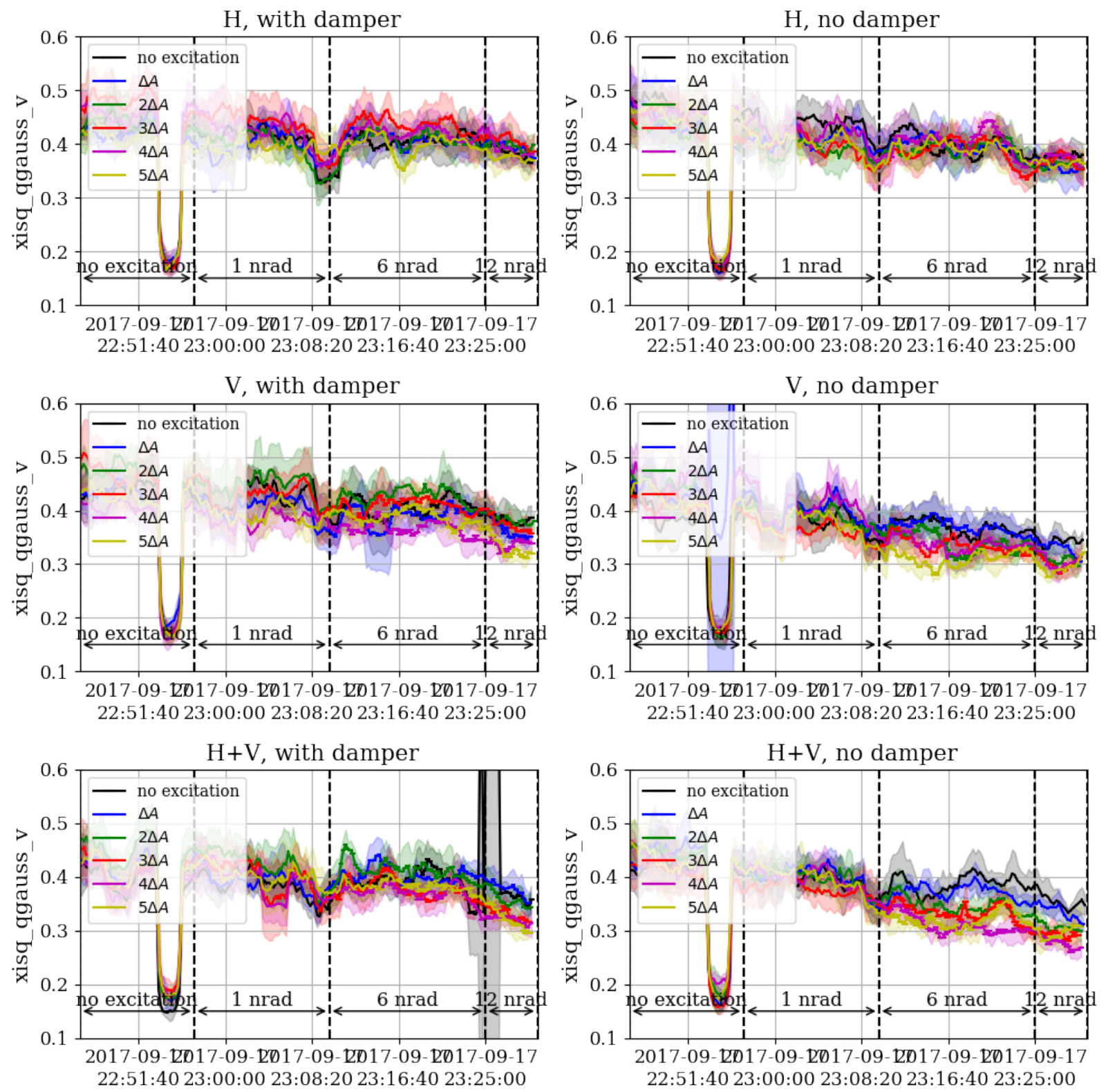

Figure 88. random excitation: normalized $\chi^{2}$ of q-Gaussian fit of the BSRT profiles (Eqn. 9) in the vertical plane measured with the BSRT together with the $1 \sigma$ standard deviation over the bunches with the same excitation amplitude $n \cdot \Delta A$ indicated as an envelope for each amplitude. For all values the moving average profiles are used without any further averaging afterwards. The change of the $\chi^{2}$ between 22:53 and 22:55 is due to the orbit shift shown in Appendix B 3 a, Figs. 72-73. 



Figure 89. random excitation: correlation coefficient between fit parameter $c$ and $a$ from q-Gaussian fit of the BSRT profiles (Eqn. 9) in the vertical plane measured with the BSRT together with the $1 \sigma$ standard deviation over the bunches with the same excitation amplitude $n \cdot \Delta A$ indicated as an envelope for each amplitude. For all values the moving average profiles are used without any further averaging afterwards. The change in correlation coefficient between 22:53 and 22:55 is due to the orbit shift shown in Appendix B 3 a, Figs. 72-73. 



Figure 90. random excitation: correlation coefficient between fit parameter $c$ and $q$ from q-Gaussian fit of the BSRT profiles (Eqn. 9) in the vertical plane measured with the BSRT together with the $1 \sigma$ standard deviation over the bunches with the same excitation amplitude $n \cdot \Delta A$ indicated as an envelope for each amplitude. For all values the moving average profiles are used without any further averaging afterwards. The change in correlation coefficient between 22:53 and 22:55 is due to the orbit shift shown in Appendix B 3 a, Figs. 72-73. 

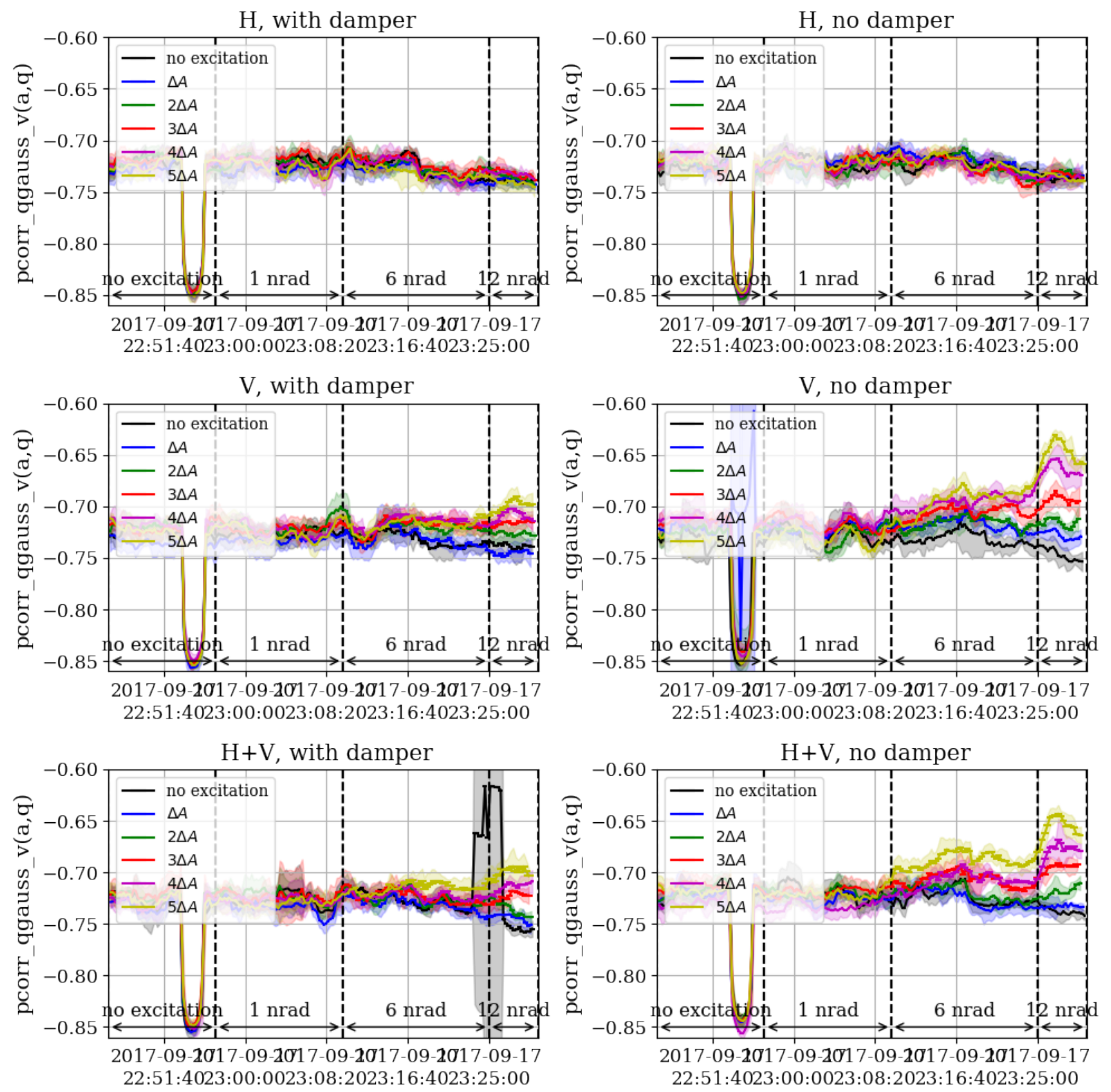

Figure 91. random excitation: correlation coefficient between fit parameter $a$ and $q$ from q-Gaussian fit of the BSRT profiles (Eqn. 9) in the vertical plane measured with the BSRT together with the $1 \sigma$ standard deviation over the bunches with the same excitation amplitude $n \cdot \Delta A$ indicated as an envelope for each amplitude. For all values the moving average profiles are used without any further averaging afterwards. The change in correlation coefficient between 22:53 and 22:55 is due to the orbit shift shown in Appendix B 3 a, Figs. 72-73. 


\section{d. random excitation: BSRT profiles of representative bunches}

no damper, no excitation
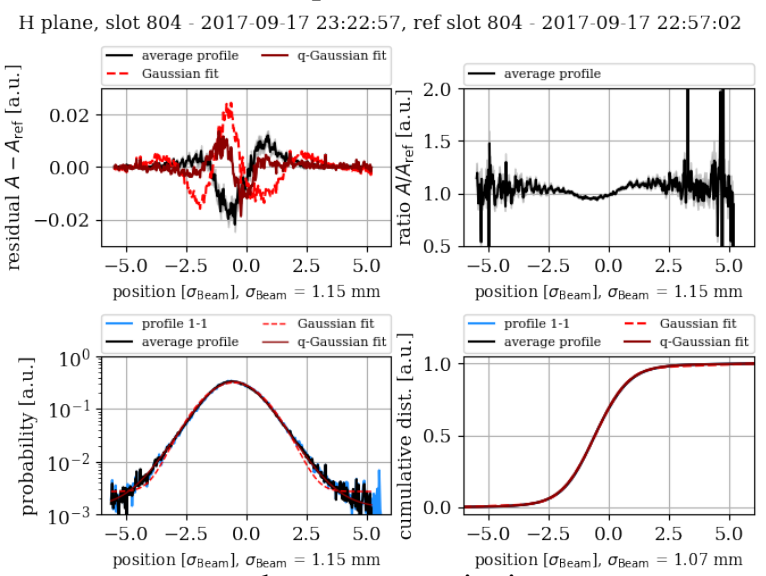
no damper, no excitation

V plane, slot 804 - 2017-09-17 23:22:57, ref slot 804 - 2017-09-17 22:57:02
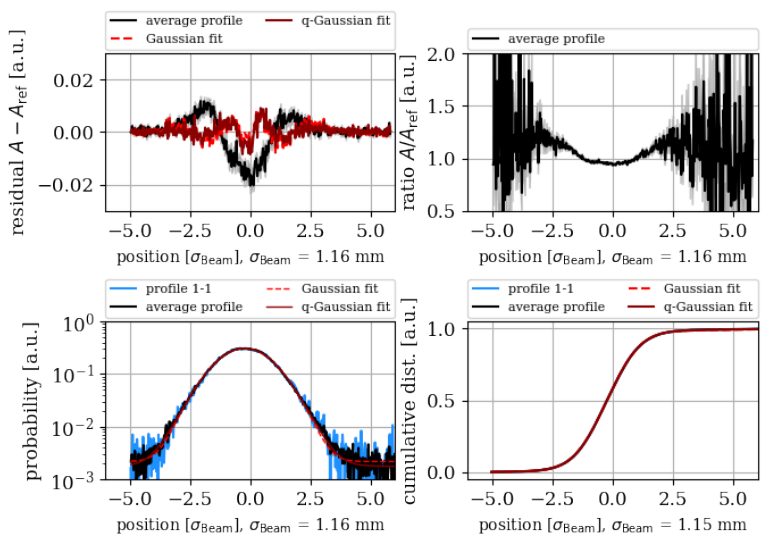

no damper, with excitation $(5 \cdot \Delta A)$ in $\mathrm{H}$
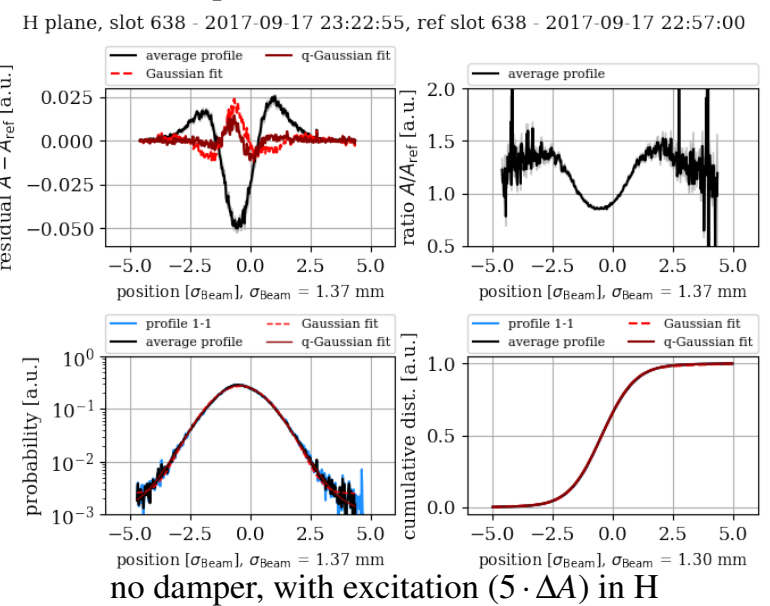

V plane, slot 638 - 2017-09-17 23:22:55, ref slot 638 - 2017-09-17 22:57:00
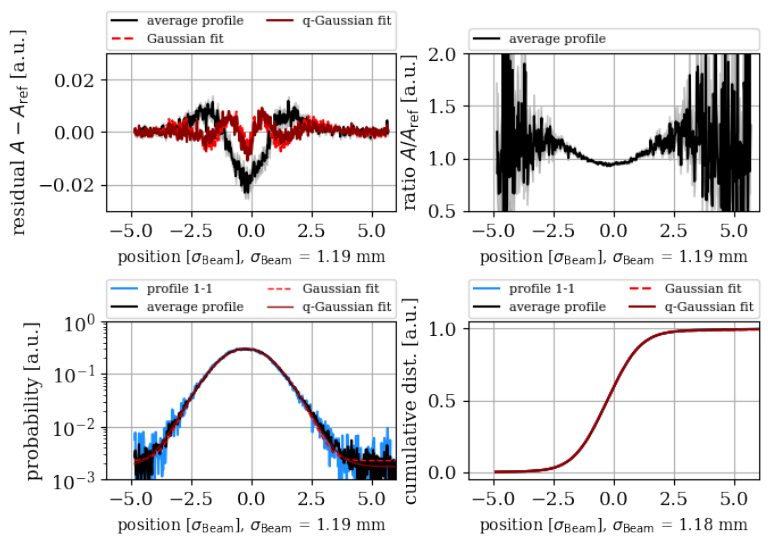

Figure 92. Random excitation: BSRT profile in the horizontal (top) and vertical (bottom) plane of one bunch without resonant excitation (left) and one bunch experiencing the maximum excitation (right) for an excitation only in H. For all bunches the damper is not active. The residual is taken in respect to the start (2017-09-17 22:57) and end (201709-17 23:22) of the random excitation with a few minutes subtracted in order to account for the moving average of the profiles over 11 time stamps. The $\mathrm{x}$-axis is the position of the BSRT image projection and not the proton beam sigma. 
no damper, no excitation

H plane, slot 1698 - 2017-09-17 23:22:46, ref slot 1698 - 2017-09-17 22:57:06
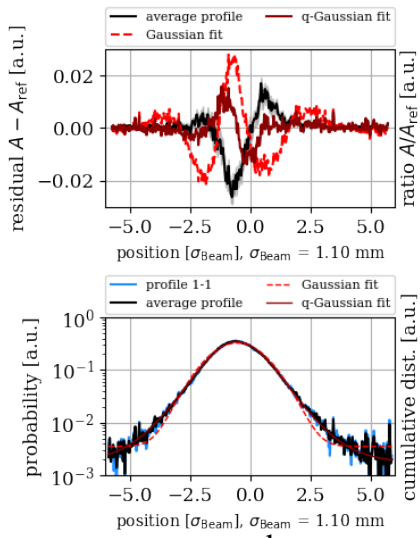
no damper, no excitation

V plane, slot 1698 - 2017-09-17 23:22:46, ref slot 1698 - 2017-09-17 22:57:06
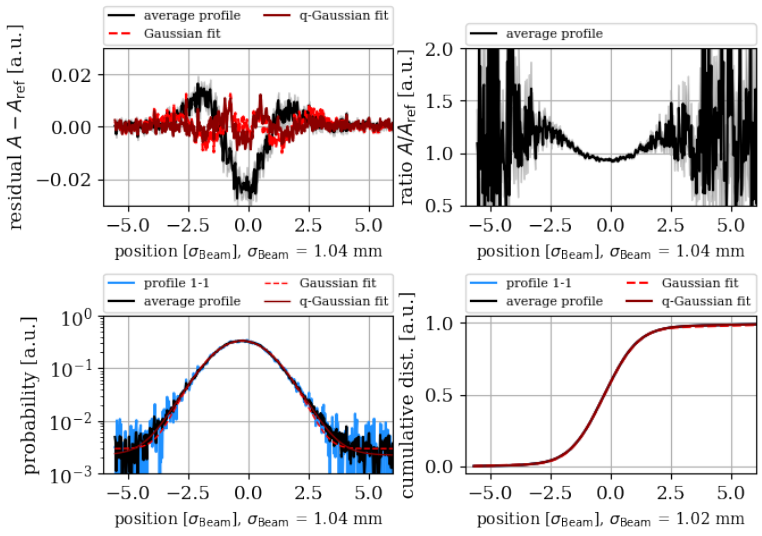
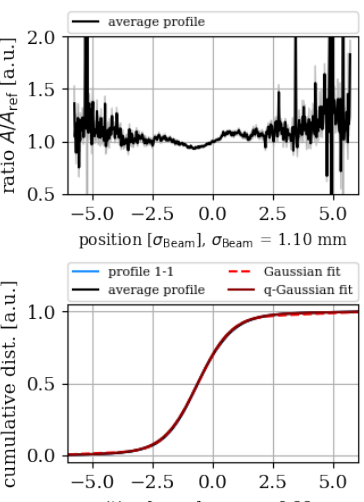

position $\left[\sigma_{\text {Beam }}\right], \sigma_{\text {Beam }}=0.99 \mathrm{~mm}$

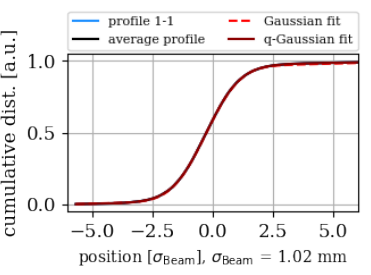

no damper, with excitation $(5 \cdot \Delta A)$ in $\mathrm{V}$

H plane, slot 1532 - 2017-09-17 23:22:44, ref slot 1532 - 2017-09-17 22:57:05


no damper, with excitation $(5 \cdot \Delta A)$ in $\mathrm{V}$

V plane, slot 1532 - 2017-09-17 23:22:44, ref slot 1532 - 2017-09-17 22:57:05
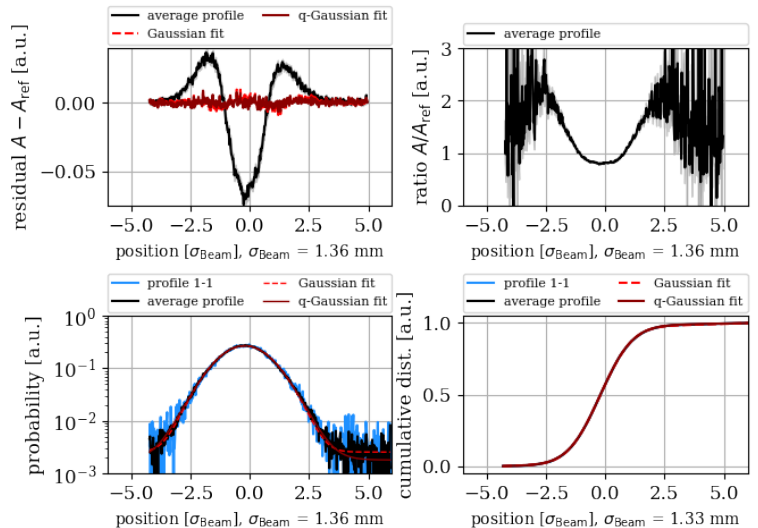

Figure 93. Random excitation: BSRT profile in the horizontal (top) and vertical (bottom) plane of one bunch without resonant excitation (left) and one bunch experiencing the maximum excitation (right) for an excitation only in V. For all bunches the damper is not active. The residual is taken in respect to the start (2017-09-17 22:57) and end (201709-17 23:22) of the random excitation with a few minutes subtracted in order to account for the moving average of the profiles over 11 time stamps. Note that $22: 56$ is also after the closed orbit shift in the vertical plane. The $\mathrm{x}$-axis is the position of the BSRT image projection and not the proton beam sigma. 
no damper, no excitation

H plane, slot 2862 - 2017-09-17 23:22:51, ref slot 2862 - 2017-09-17 22:57:10

no damper, no excitation

V plane, slot 2862 - 2017-09-17 23:22:51, ref slot 2862 - 2017-09-17 22:57:10
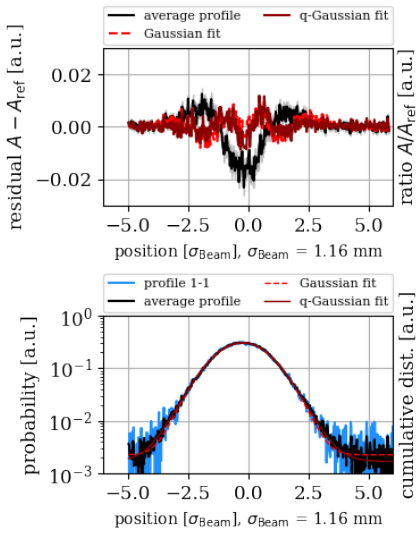
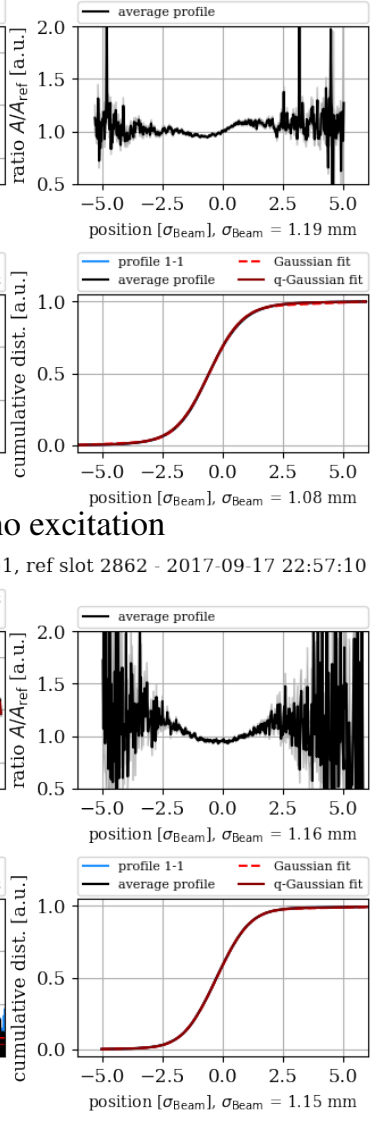

no damper, with excitation $(5 \cdot \Delta A)$ in $\mathrm{H}+\mathrm{V}$

H plane, slot 2696 - 2017-09-17 23:22:49, ref slot 2696 - 2017-09-17 22:57:09
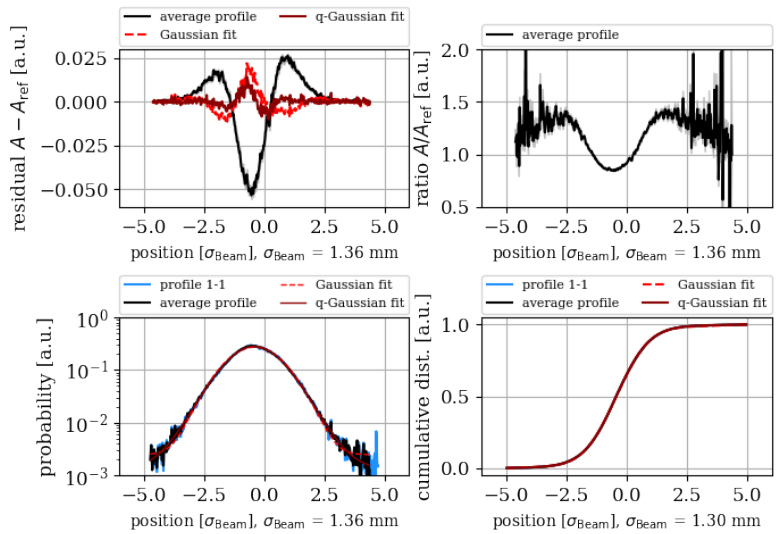

no damper, with excitation $(5 \cdot \Delta A)$ in $\mathrm{H}+\mathrm{V}$

V plane, slot 2696 - 2017-09-17 23:22:49, ref slot 2696 - 2017-09-17 22:57:09
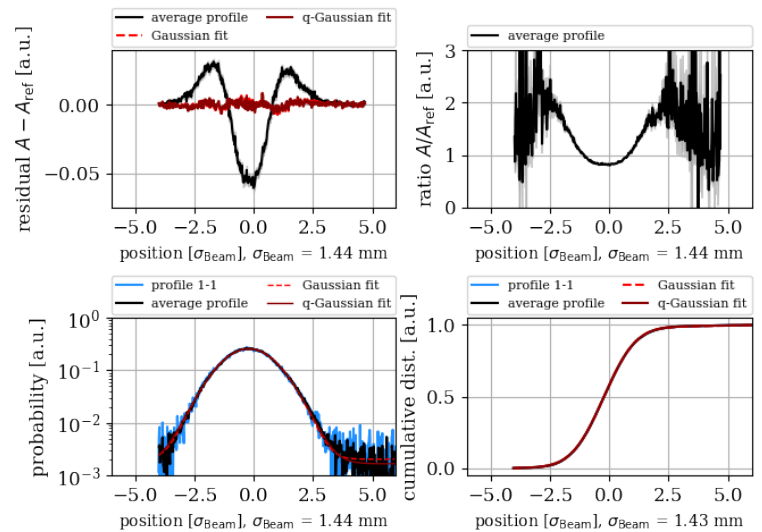

Figure 94. Random excitation: BSRT profile in the horizontal (top) and vertical (bottom) plane of one bunch without resonant excitation (left) and one bunch experiencing the maximum excitation (right) for an excitation only in $\mathrm{H}+\mathrm{V}$. For all bunches the damper is not active. The residual is taken in respect to the start (2017-09-17 22:57) and end (201709-17 23:22) of the random excitation with a few minutes subtracted in order to account for the moving average of the profiles over 11 time stamps. Note that $22: 56$ is also after the closed orbit shift in the vertical plane. The $\mathrm{x}$-axis is the position of the BSRT image projection and not the proton beam sigma. 
with damper, no excitation

H plane, slot 442 - 2017-09-17 23:22:52, ref slot 442 - 2017-09-17 22:57:11
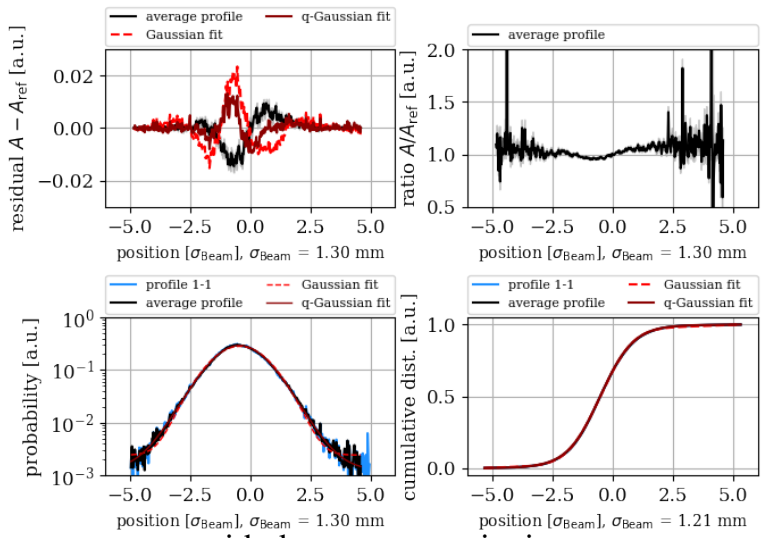

with damper, no excitation

V plane, slot 442 - 2017-09-17 23:22:52, ref slot 442 - 2017-09-17 22:57:11


with damper, with excitation $(5 \cdot \Delta A)$ in $\mathrm{H}$
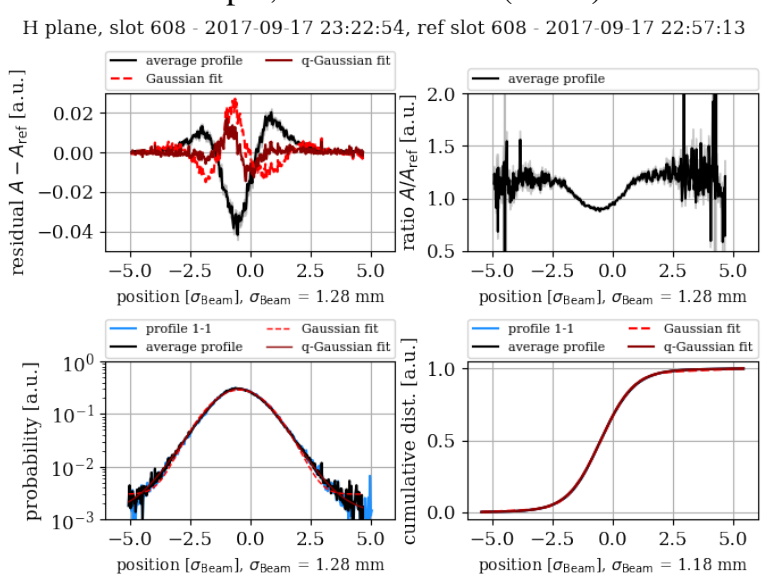

position $\left[\sigma_{\text {Beam }}\right], \sigma_{\text {Beam }}=1.18 \mathrm{~mm}$ with damper, with excitation $(5 \cdot \Delta A)$ in $\mathrm{H}$

V plane, slot 608 - 2017-09-17 23:22:54, ref slot 608 - 2017-09-17 22:57:13


Figure 95. Random excitation: BSRT profile in the horizontal (top) and vertical (bottom) plane of one bunch without resonant excitation (left) and one bunch experiencing the maximum excitation (right) for an excitation only in H. For all bunches the damper is active. The residual is taken in respect to the start (2017-09-17 22:57) and end (2017-09-17 23:22) of the random excitation with a few minutes subtracted in order to account for the moving average of the profiles over 11 time stamps. The $\mathrm{x}$-axis is the position of the BSRT image projection and not the proton beam sigma. 
with damper, no excitation

H plane, slot 1336 - 2017-09-17 23:22:57, ref slot 1336 - 2017-09-17 22:57:03
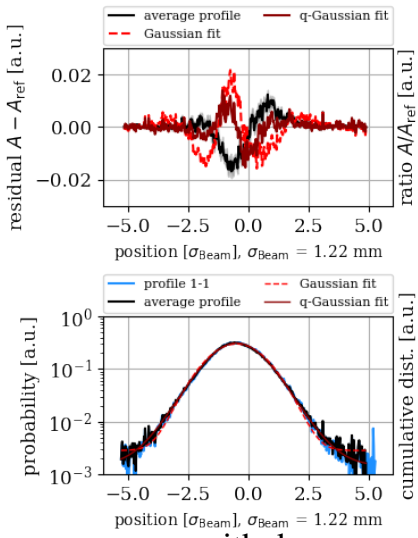

with damper, no excitation

V plane, slot 1336 - 2017-09-17 23:22:57, ref slot 1336 - 2017-09-17 22:57:03

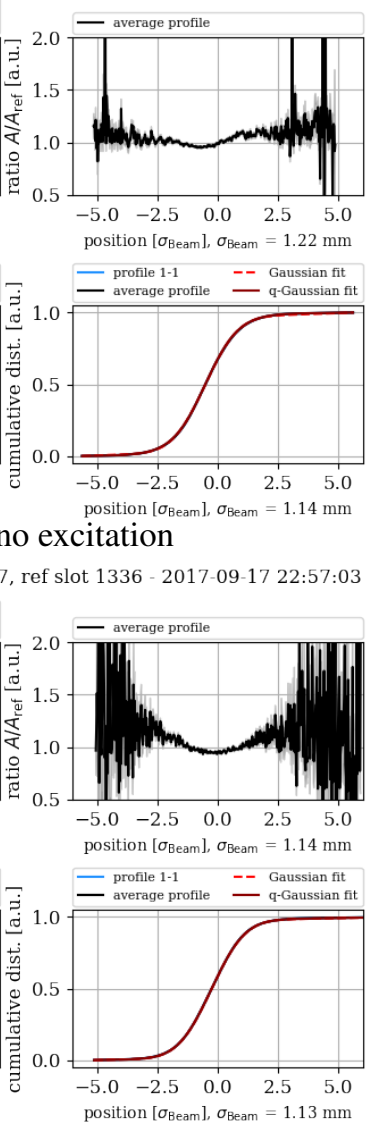

with damper, with excitation $(5 \cdot \Delta A)$ in $\mathrm{V}$

H plane, slot 1502 - 2017-09-17 23:22:44, ref slot 1502 - 2017-09-17 22:57:04
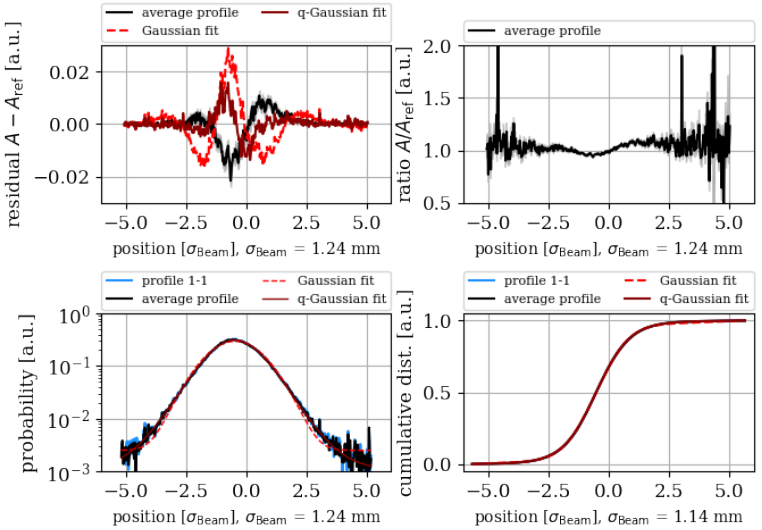
position $\left[\sigma_{\text {Beam }}\right], \sigma_{\text {Beam }}=1.24 \mathrm{~mm}$
with damper, with excitation $(5 \cdot \Delta A)$ in $\mathrm{V}$

V plane, slot 1502 - 2017-09-17 23:22:44, ref slot 1502 - 2017-09-17 22:57:04
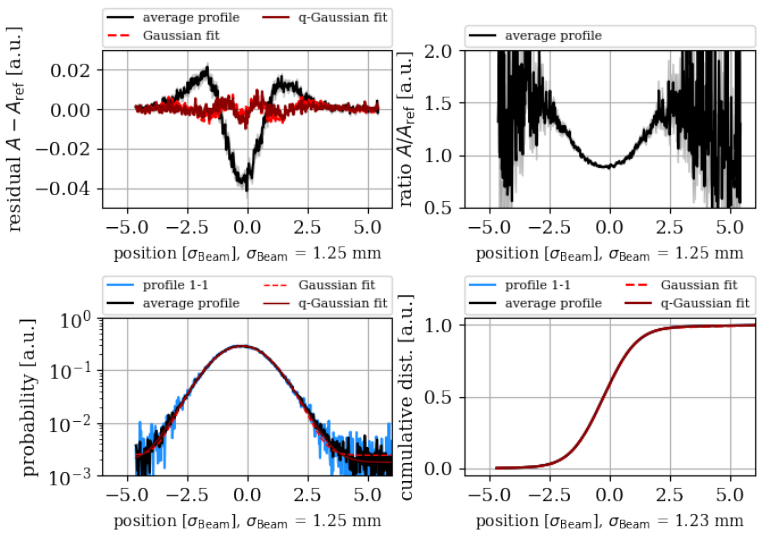
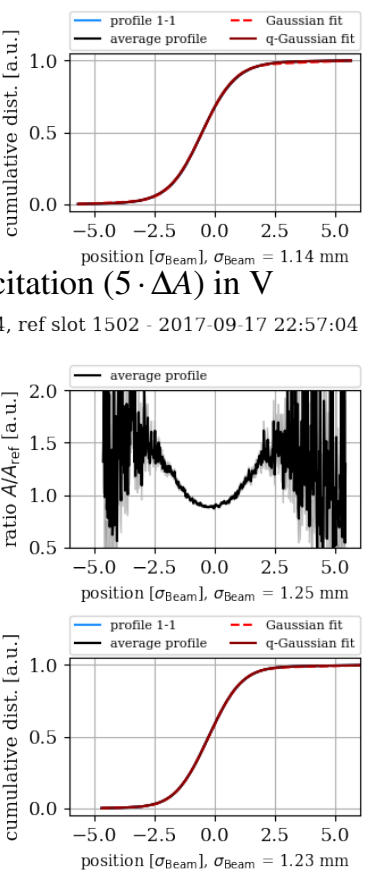

Figure 96. Random excitation: BSRT profile in the horizontal (top) and vertical (bottom) plane of one bunch without resonant excitation (left) and one bunch experiencing the maximum excitation (right) for an excitation only in V. For all bunches the damper is active. The residual is taken in respect to the start (2017-09-17 22:57) and end (2017-09-17 23:22) of the random excitation with a few minutes subtracted in order to account for the moving average of the profiles over 11 time stamps. Note that 22:56 is also after the closed orbit shift in the vertical plane. The $\mathrm{x}$-axis is the position of the BSRT image projection and not the proton beam sigma. 
with damper, no excitation

with damper, no excitation

V plane, slot 2500 - 2017-09-17 23:22:47, ref slot 2500 - 2017-09-17 22:57:07
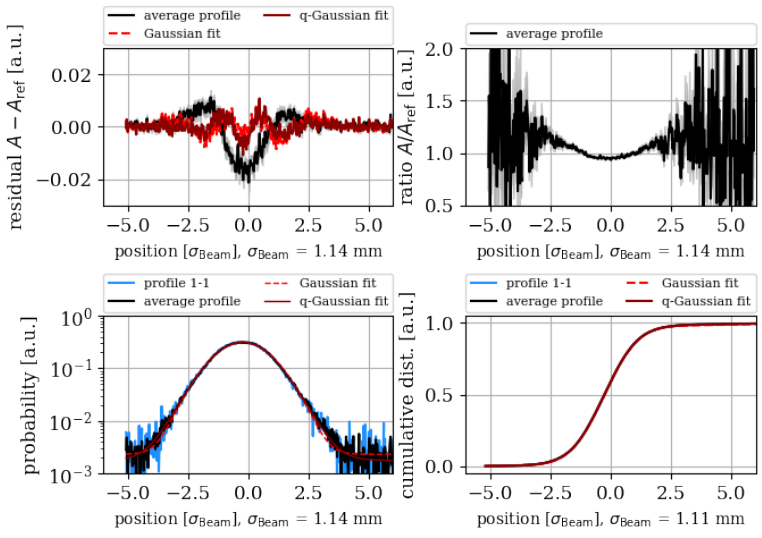
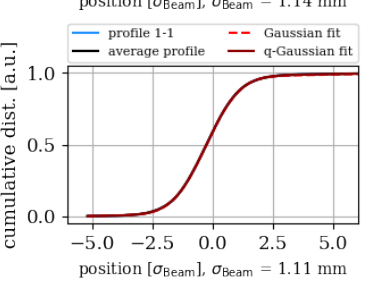

with damper, with excitation $(5 \cdot \Delta A)$ in $\mathrm{H}+\mathrm{V}$

H plane, slot 2666 - 2017-09-17 23:22:49, ref slot 2666 - 2017-09-17 22:57:08
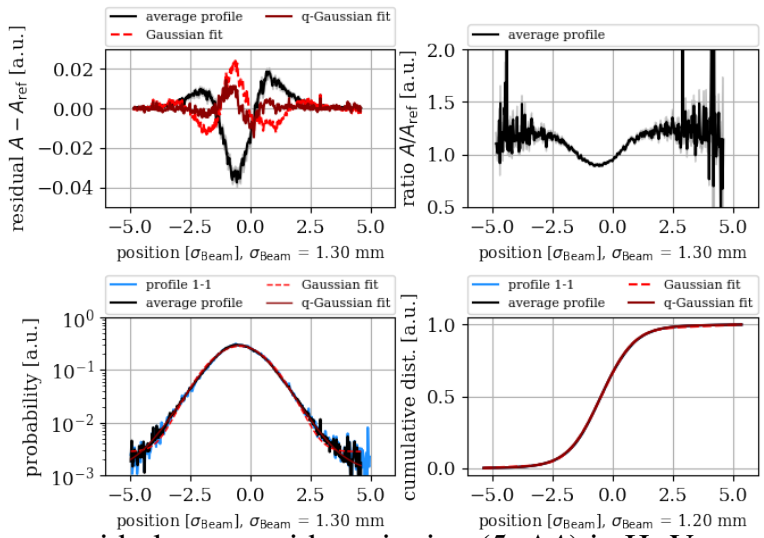

position $\left[\sigma_{\text {Beam }}\right], \sigma_{\text {Beam }}=1.30 \mathrm{~mm}$.

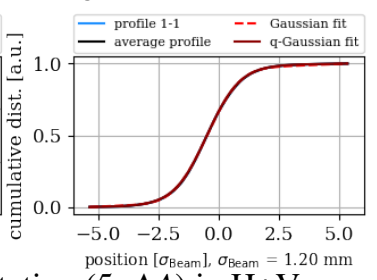

position $\left[\sigma_{\text {Beam }}\right], \sigma_{\text {Beam }}=1.20 \mathrm{~mm}$

with damper, with excitation $(5 \cdot \Delta A)$ in $\mathrm{H}+\mathrm{V}$

V plane, slot 2666 - 2017-09-17 23:22:49, ref slot 2666 - 2017-09-17 22:57:08
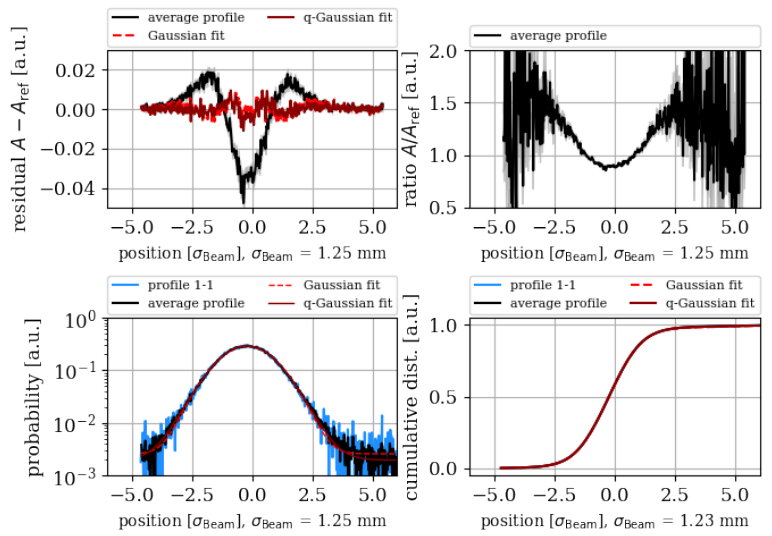

Figure 97. Random excitation: BSRT profile in the horizontal (top) and vertical (bottom) plane of one bunch without resonant excitation (left) and one bunch experiencing the maximum excitation (right) for an excitation only in $\mathrm{H}+\mathrm{V}$. For all bunches the damper is active. The residual is taken in respect to the start (2017-09-17 22:57) and end (201709-17 23:22) of the random excitation with a few minutes subtracted in order to account for the moving average of the profiles over 11 time stamps. Note that $22: 56$ is also after the closed orbit shift in the vertical plane. The $\mathrm{x}$-axis is the position of the BSRT image projection and not the proton beam sigma. 


\section{4. random excitation: FMA Analysis for 2017 optics and tune}
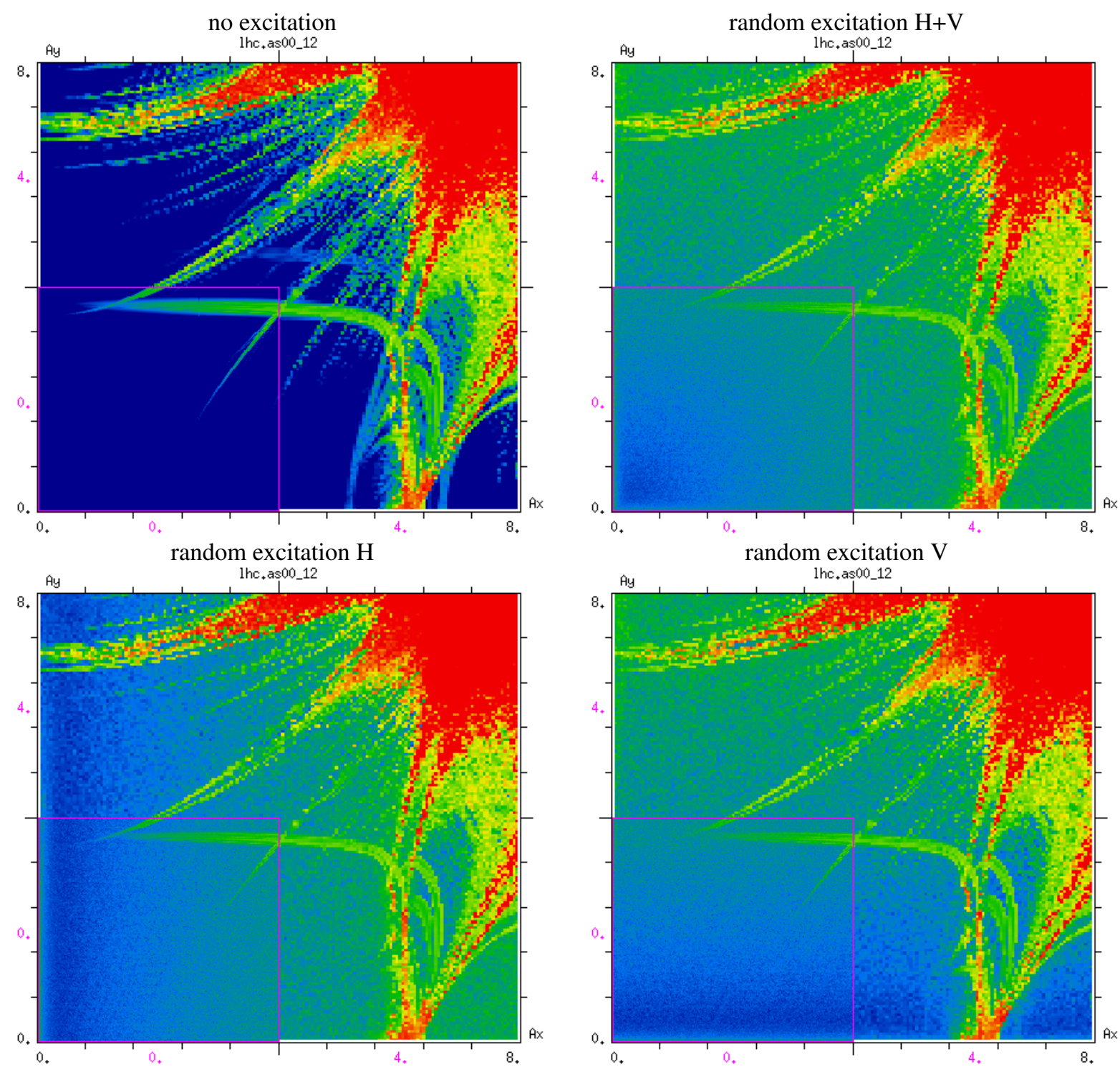

Figure 98. FMA analysis in amplitude space for a random excitation based on the 2017 injection optics with no machine errors and a tune of $(62.27,60.295)$. The excitation is $1 \mathrm{nrad}$ in each/both planes. The random excitation excites all frequencies in contrast to any $n^{\text {th }}$ turn pulsing acting only on certain resonances. 


\section{Appendix C: Additional figures for fill 6220, $8^{\text {th }}$ turn pusling}

\section{1. $8^{\text {th }}$ turn pulsing: beam losses measured with FBCT}
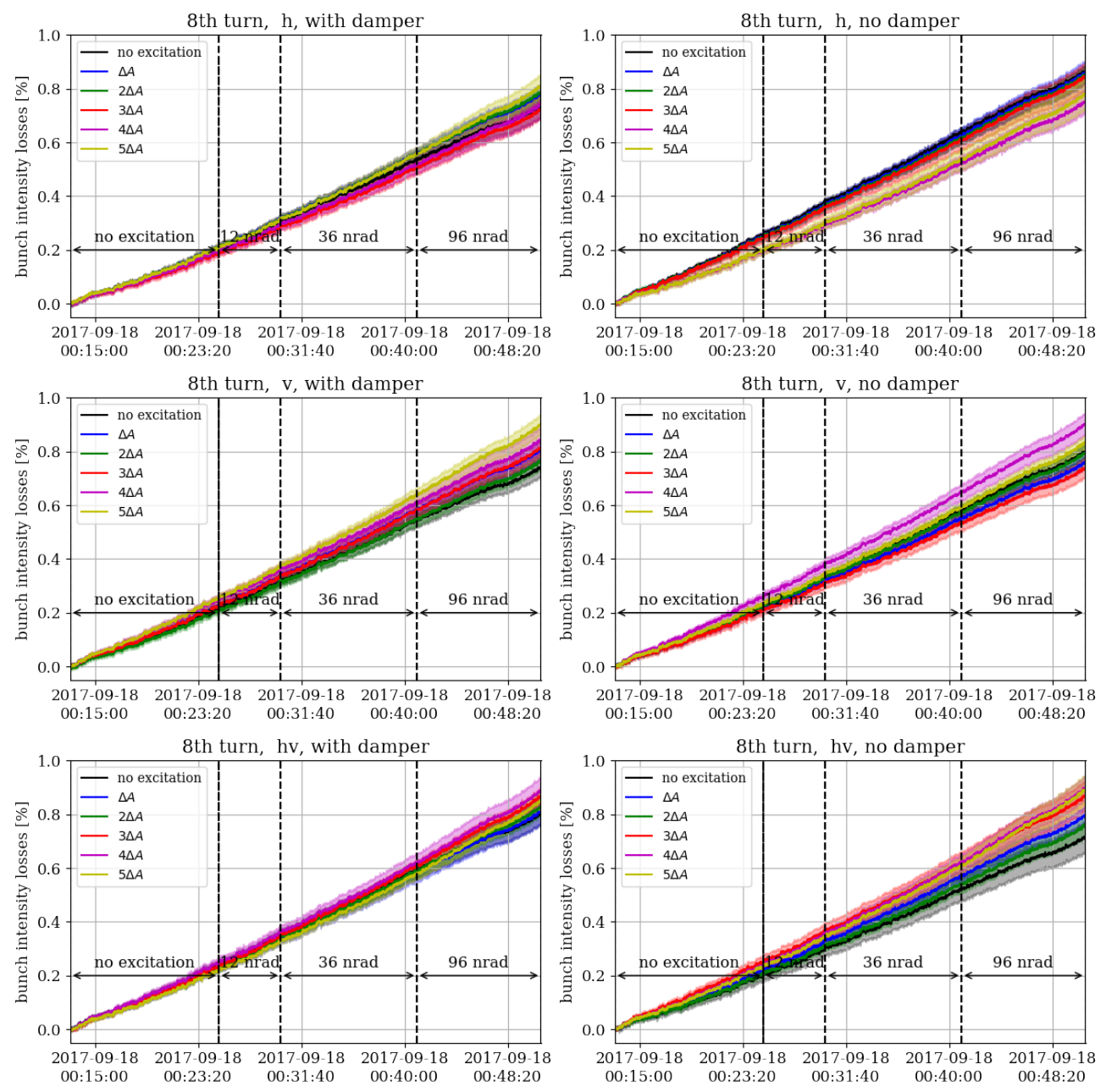

Figure 99. $8^{\text {th }}$ turn pulsing: Relative bunch intensity losses measured with the FBCTs (Eqn. 3). The average over the group of 6 bunches experiencing the same excitation amplitude is indicated by a solid line together with the $1 \sigma$ standard deviation over the four bunches indicated as an envelope in the same color. The maximum excitation amplitudes are indicated with black arrows. 


\section{2. $8^{\text {th }}$ turn pulsing: BSRT emittance as logged in the LHC Logging database}


Figure 100. $8^{\text {th }}$ turn pulsing: relative emittance in the horizontal plane measured with the BSRT (Gaussian fit) and as defined in Eqn. 24 together with the $1 \sigma$ standard deviation over the bunches with the same excitation amplitude $n \cdot \Delta A$ indicated as an envelope for each amplitude. For all emittance values a moving average over 10 time stamps is performed. 

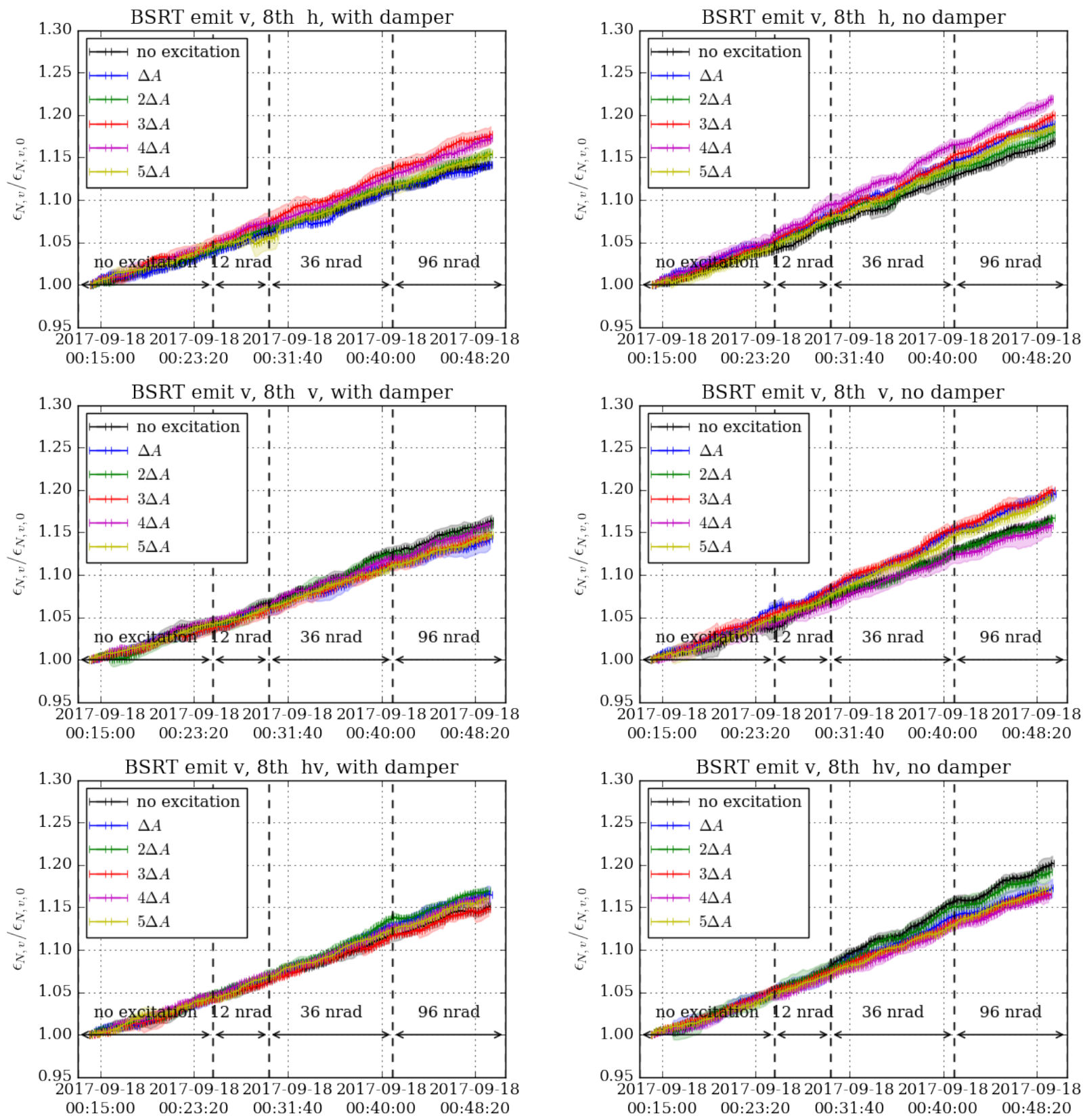

Figure 101. $8^{\text {th }}$ turn pulsing: relative emittance in the vertical plane measured with the BSRT (Gaussian fit) and as defined in Eqn. 24 together with the $1 \sigma$ standard deviation over the bunches with the same excitation amplitude $n \cdot \Delta A$ indicated as an envelope for each amplitude. For all emittance values a moving average over 10 time stamps is performed. 

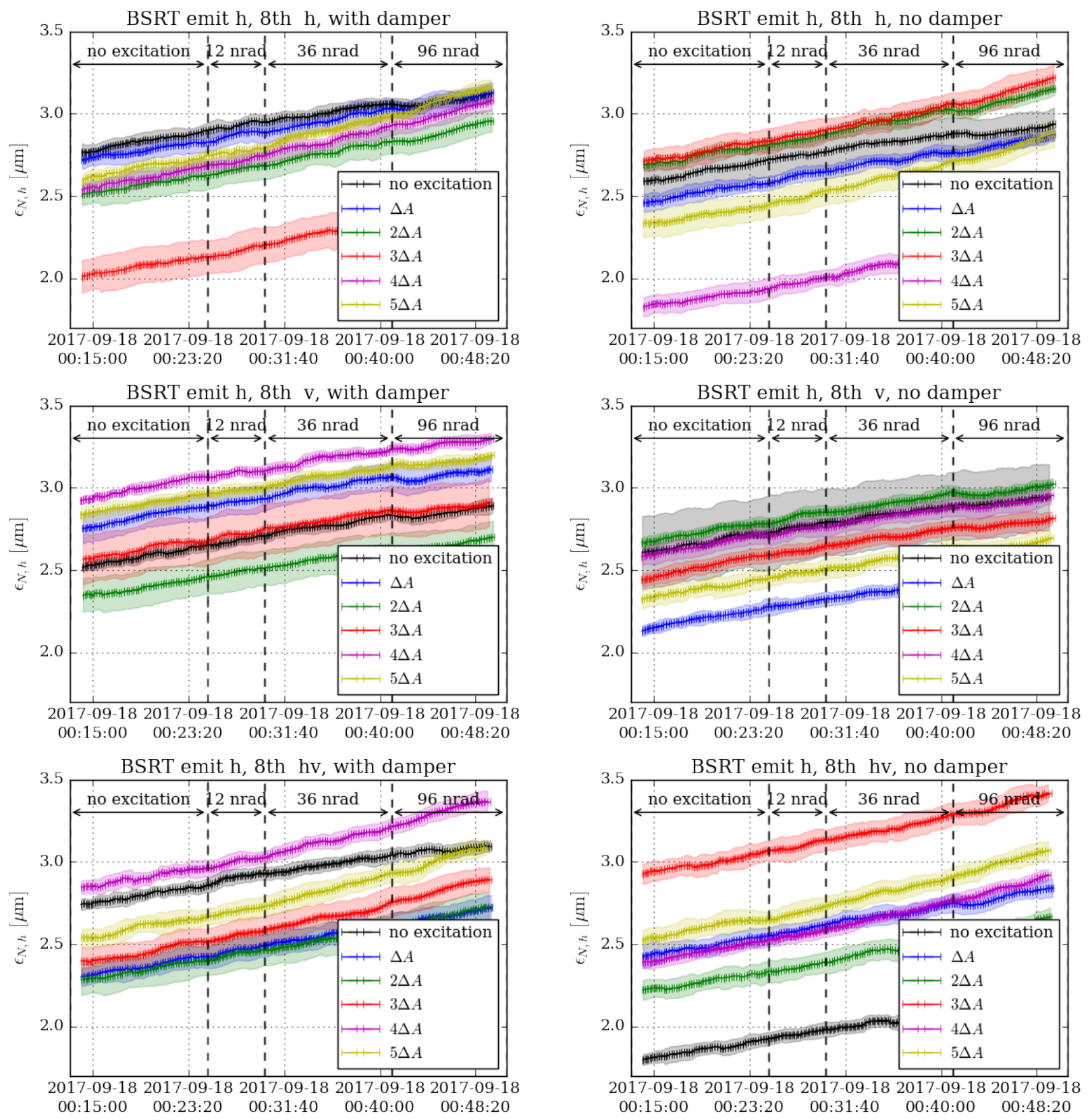

Figure 102. $8^{\text {th }}$ turn pulsing: emittance in the horizontal plane measured with the BSRT (Gaussian fit) and as defined in Eqn. 22 together with the $1 \sigma$ standard deviation over the bunches with the same excitation amplitude $n \cdot \Delta A$ indicated as an envelope for each amplitude. For all emittance values a moving average over 10 time stamps is performed. 



Figure 103. $8^{\text {th }}$ turn pulsing: relative emittance in the vertical plane measured with the BSRT (Gaussian fit) and as defined in Eqn. 22 together with the $1 \sigma$ standard deviation over the bunches with the same excitation amplitude $n \cdot \Delta A$ indicated as an envelope for each amplitude. For all emittance values a moving average over 10 time stamps is performed. 

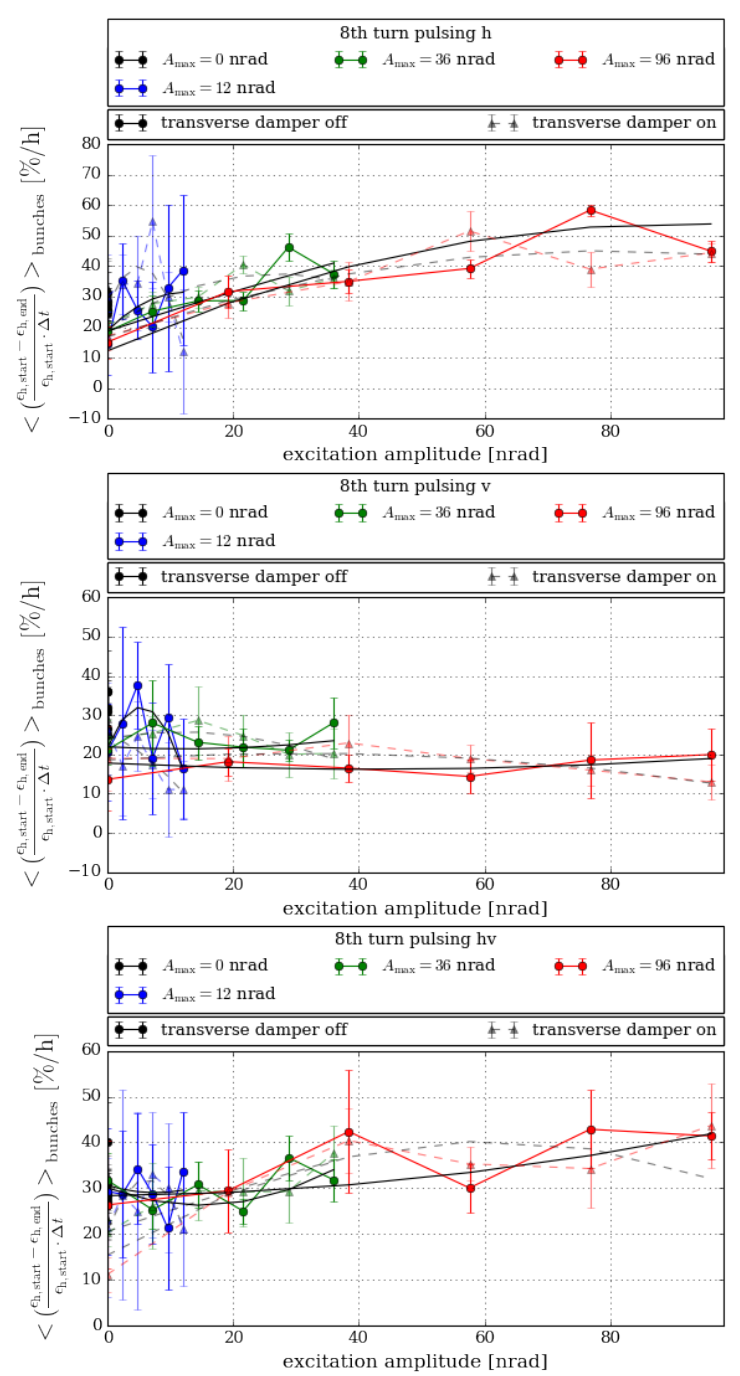
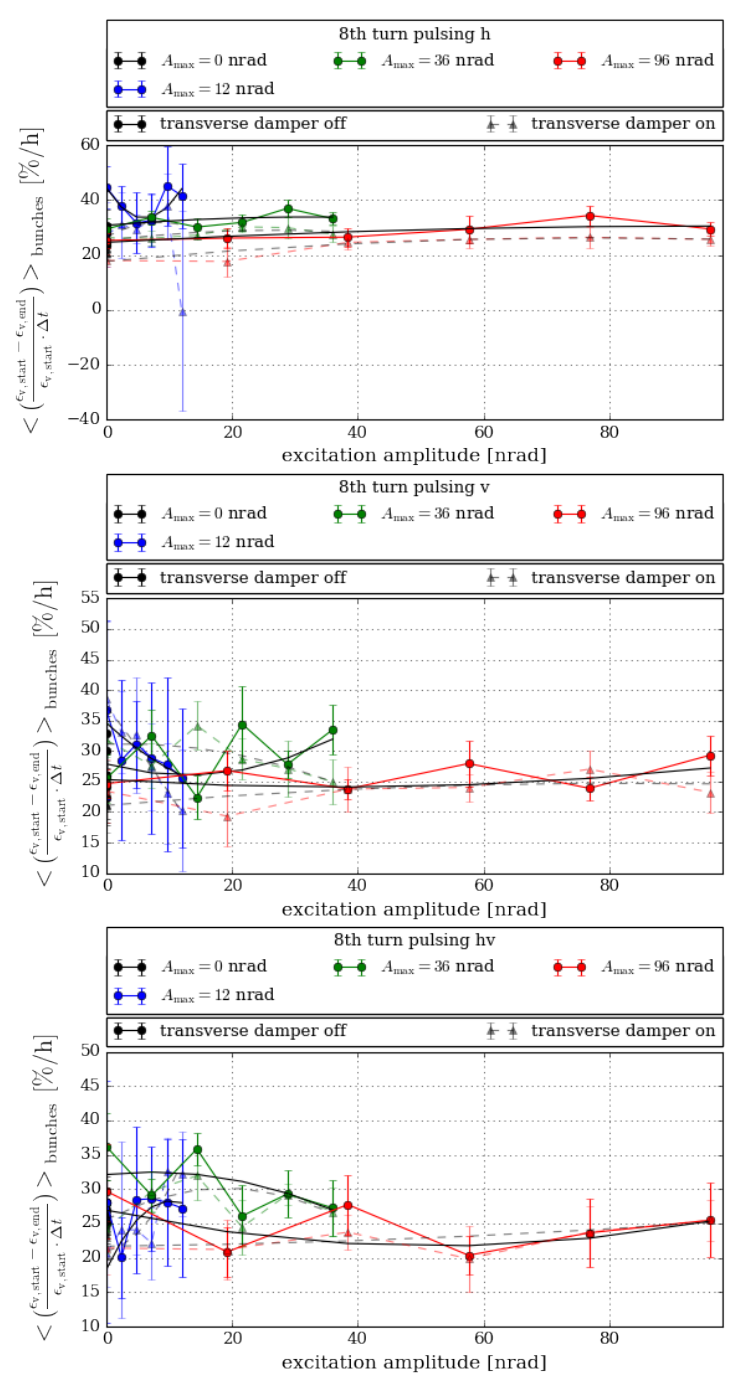

Figure 104. $8^{\text {th }}$ turn pulsing: Average emittance growth rate in the horizontal (left) and vertical (right) plane for an excitation in $\mathrm{H}$ (top) and in $\mathrm{H}+\mathrm{V}$ (bottom) measured with the BSRT and as defined in Eqn. 25 using the emittance $\varepsilon$ from the LHC Logging database. The measurements for the different maximum excitation amplitudes $A_{\max }$ are indicated in different colors. The bunches for which the transverse damper is active are shown with solid dots and the bunches for which the transverse damper is not active are shown with slightly transparent triangles. The quadratic fit to the emittance growth rate is indicated with a solid line for the bunches with transverse damper active and with a dashed line for the bunches with the transverse damper not active. For all emittance values a moving average over 10 time stamps is performed. 


\section{3. $8^{\text {th }}$ turn pulsing: BSRT profile analysis}

\section{a. $\quad 8^{\text {th }}$ turn pulsing: BSRT profiles - statistical parameters}
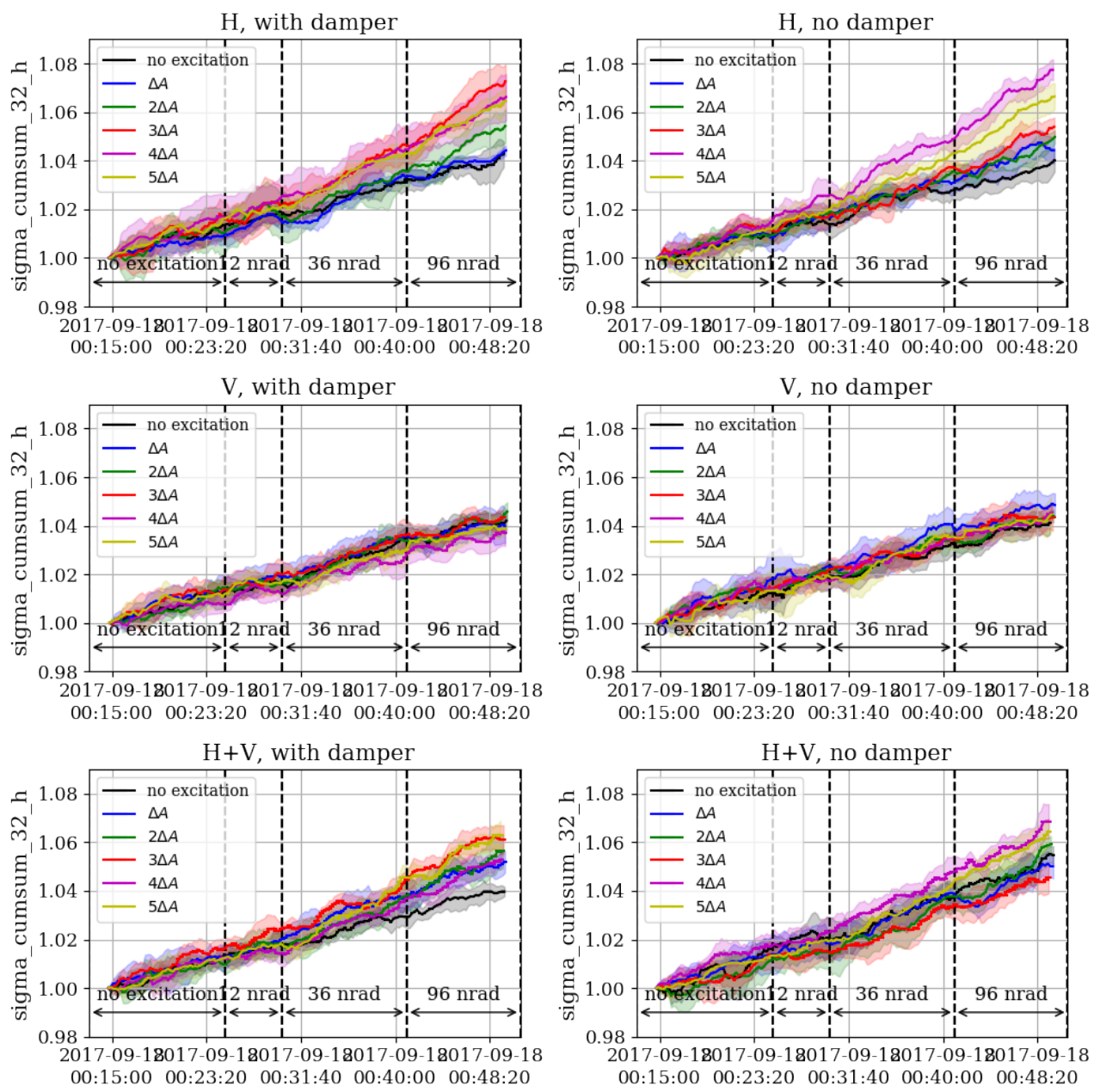

Figure 105. $8^{\text {th }}$ turn pulsing: relative beam sigma calculated over the cumulative sum (Eqn. 17) in the horizontal plane measured with the BSRT together with the $1 \sigma$ standard deviation over the bunches with the same excitation amplitude $n \cdot \Delta A$ indicated as an envelope for each amplitude. For all values a moving average over 10 time stamps is performed and the average, explicitly not moving average, profiles are used. There is no dependence of the beam sigma on the excitation amplitude $n \cdot \Delta A$. 



Figure 106. $8^{\text {th }}$ turn pulsing: relative beam sigma calculated over the cumulative sum (Eqn. 17) in the vertical plane measured with the BSRT together with the $1 \sigma$ standard deviation over the bunches with the same excitation amplitude $n \cdot \Delta A$ indicated as an envelope for each amplitude. For all beam sigma values a moving average over 10 time stamps is performed and the average, explicitly not moving average, profiles are used. A clear excitation amplitude dependent emittance growth for pulsing in $\mathrm{V}$ and $\mathrm{H}+\mathrm{V}$ is visible. 

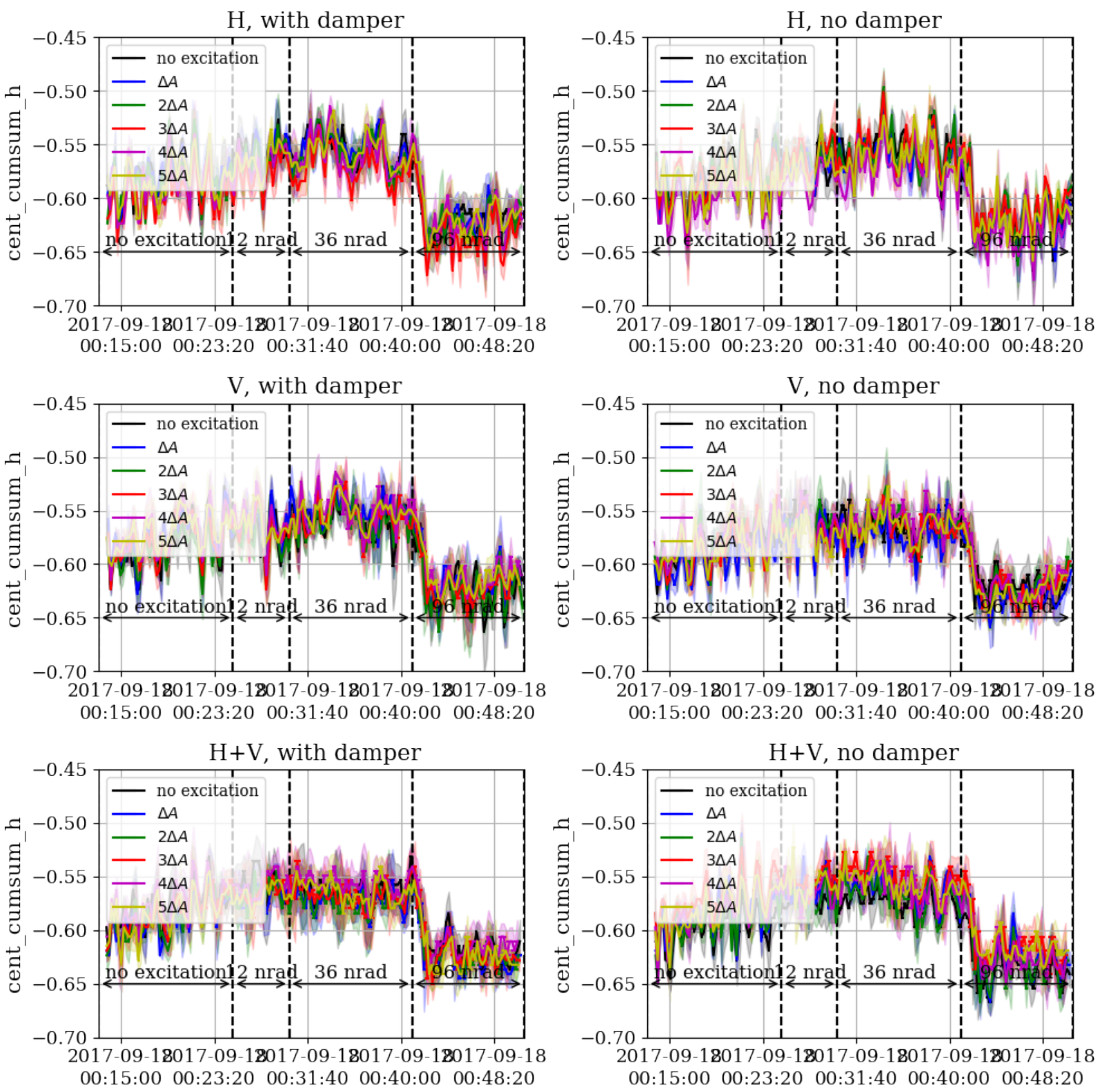

Figure 107. $8^{\text {th }}$ turn pulsing: centroid calculated over the cumulative sum (Eqn. 17) in the horizontal plane measured with the BSRT together with the $1 \sigma$ standard deviation over the bunches with the same excitation amplitude $n$. $\Delta A$ indicated as an envelope for each amplitude. No moving average is taken in order to show the un-smoothed oscillations. 



Figure 108. $8^{\text {th }}$ turn pulsing: centroid calculated over the cumulative sum (Eqn. 17) in the vertical plane measured with the BSRT together with the $1 \sigma$ standard deviation over the bunches with the same excitation amplitude $n \cdot \Delta A$ indicated as an envelope for each amplitude. No moving average is taken in order to show the un-smoothed oscillations. 
b. $8^{\text {th }}$ turn pulsing: BSRT profiles - q-Gaussian fit parameters in the horizontal plane


Figure 109. $8^{\text {th }}$ turn pulsing: relative beam sigma from q-Gaussian fit of the BSRT profiles (Eqn. 9) in the horizontal plane measured with the BSRT together with the $1 \sigma$ standard deviation over the bunches with the same excitation amplitude $n \cdot \Delta A$ indicated as an envelope for each amplitude. For all values the moving average profiles are used without any further averaging afterwards. 



Figure 110. $8^{\text {th }}$ turn pulsing: relative fit parameter $q$ from q-Gaussian fit of the BSRT profiles (Eqn. 9) in the horizontal plane measured with the BSRT together with the $1 \sigma$ standard deviation over the bunches with the same excitation amplitude $n \cdot \Delta A$ indicated as an envelope for each amplitude. For all values the moving average profiles are used without any further averaging afterwards. 

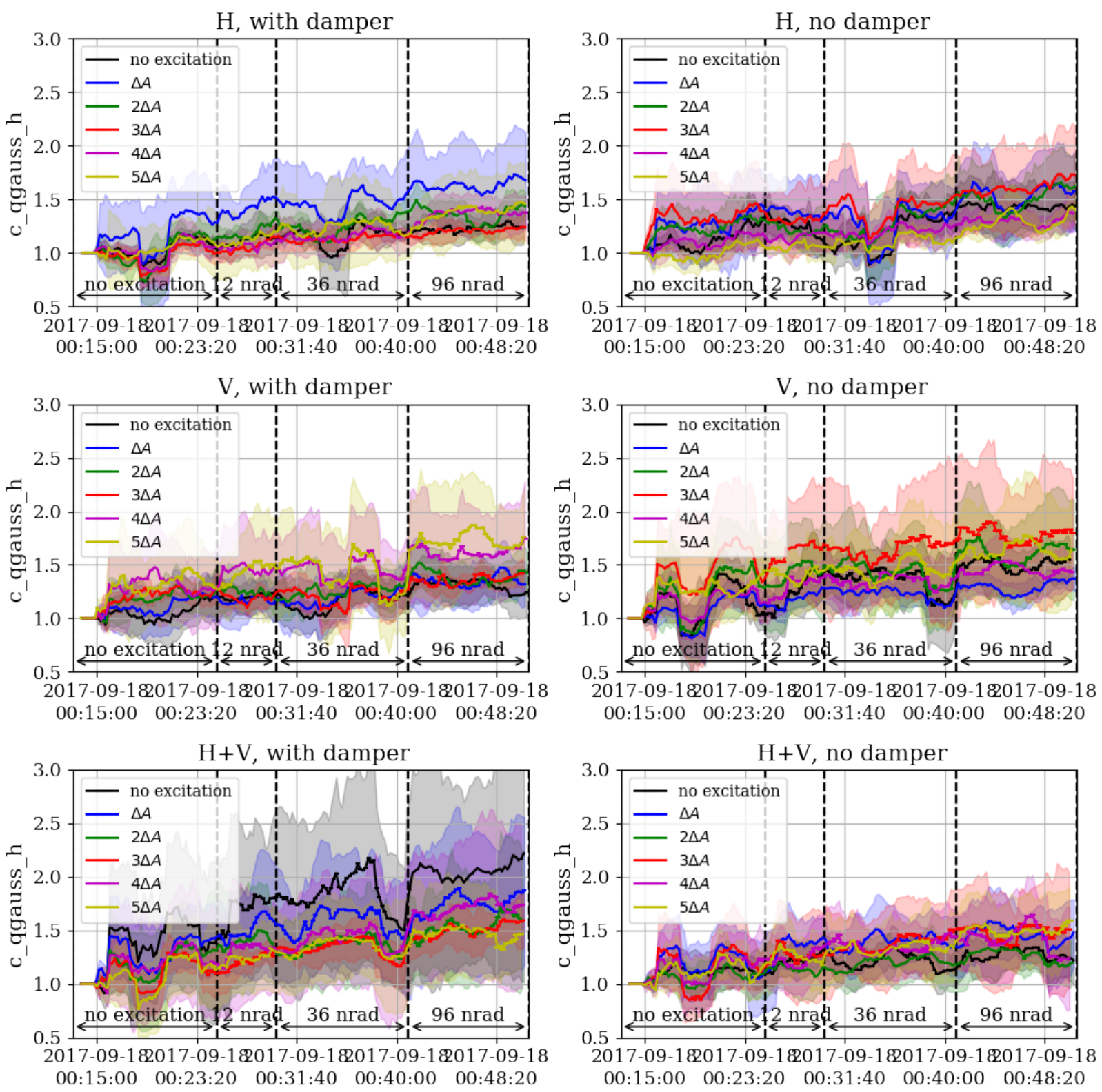

Figure 111. $8^{\text {th }}$ turn pulsing: relative fit parameter $c$ from q-Gaussian fit of the BSRT profiles (Eqn. 9) in the horizontal plane measured with the BSRT together with the $1 \sigma$ standard deviation over the bunches with the same excitation amplitude $n \cdot \Delta A$ indicated as an envelope for each amplitude. For all values the moving average profiles are used without any further averaging afterwards. 

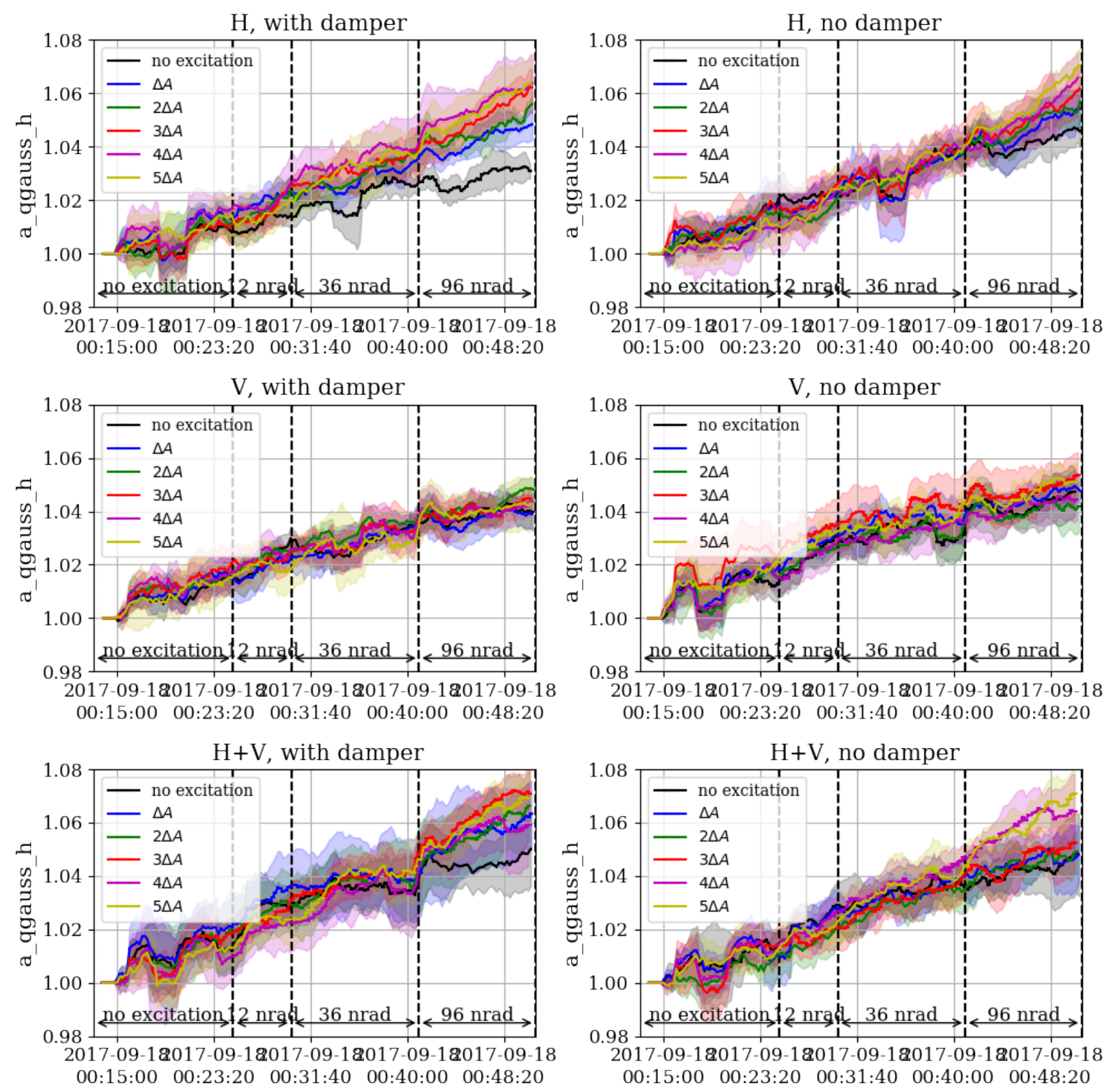

Figure 112. $8^{\text {th }}$ turn pulsing: relative fit parameter $a$ from q-Gaussian fit of the BSRT profiles (Eqn. 9) in the horizontal plane measured with the BSRT together with the $1 \sigma$ standard deviation over the bunches with the same excitation amplitude $n \cdot \Delta A$ indicated as an envelope for each amplitude. For all values the moving average profiles are used without any further averaging afterwards. 



Figure 113. $8^{\text {th }}$ turn pulsing: fit parameter $q$ from q-Gaussian fit of the BSRT profiles (Eqn. 9) in the horizontal plane measured with the BSRT together with the $1 \sigma$ standard deviation over the bunches with the same excitation amplitude $n \cdot \Delta A$ indicated as an envelope for each amplitude. For all values the moving average profiles are used without any further averaging afterwards. 

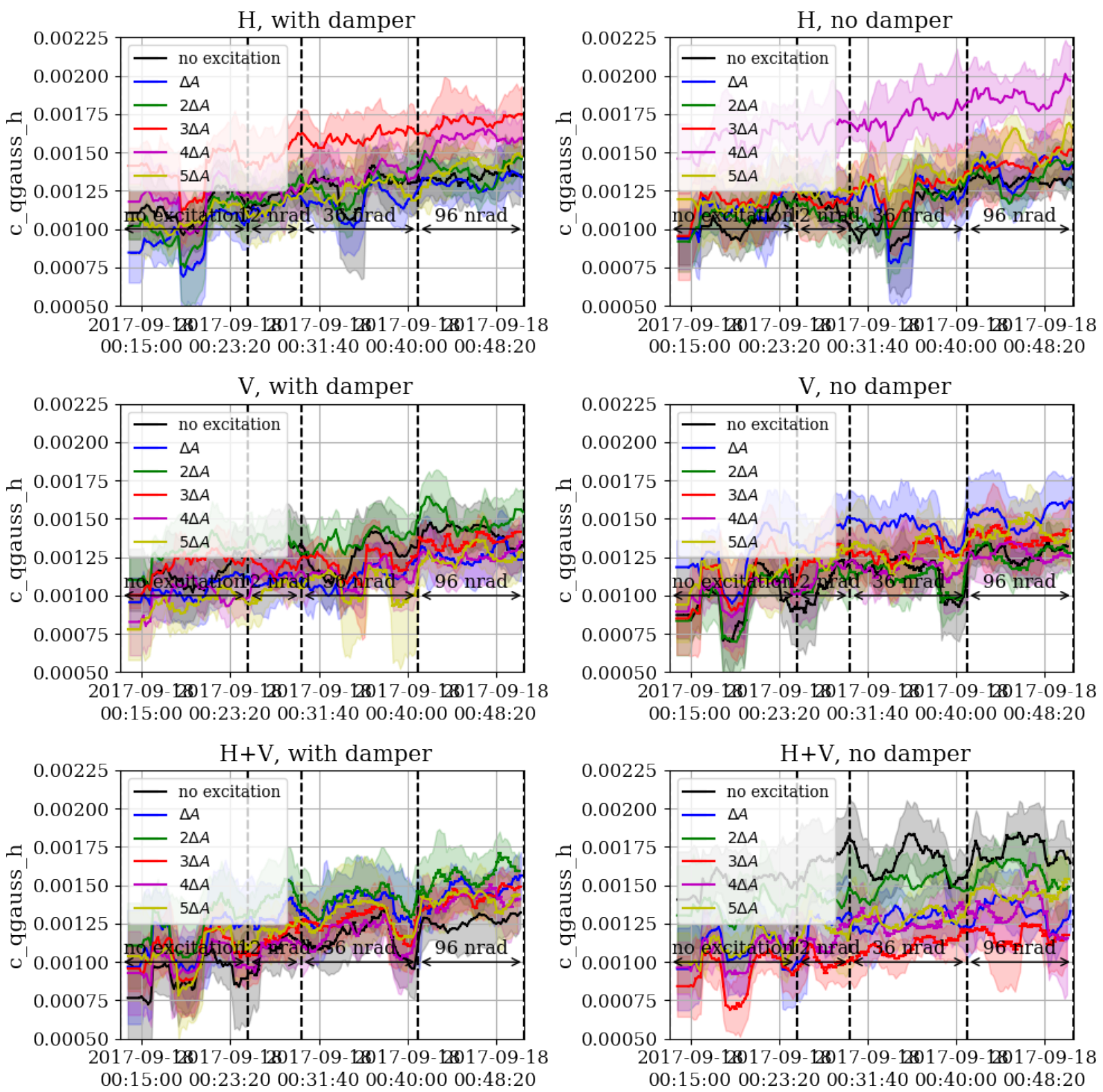

Figure 114. $8^{\text {th }}$ turn pulsing: fit parameter $c$ from q-Gaussian fit of the BSRT profiles (Eqn. 9) in the horizontal plane measured with the BSRT together with the $1 \sigma$ standard deviation over the bunches with the same excitation amplitude $n \cdot \Delta A$ indicated as an envelope for each amplitude. For all values the moving average profiles are used without any further averaging afterwards. 

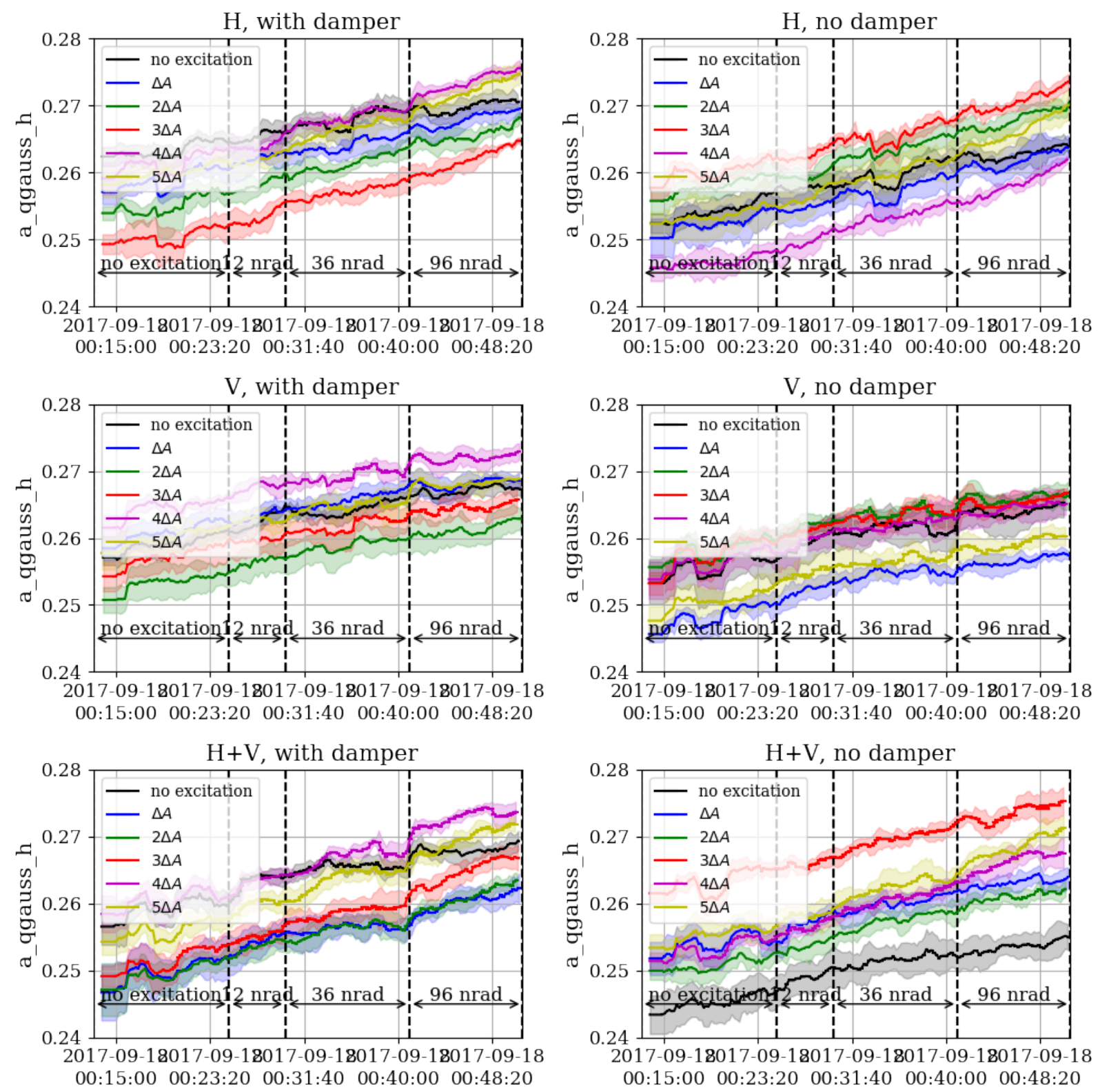

Figure 115. $8^{\text {th }}$ turn pulsing: fit parameter $a$ from q-Gaussian fit of the BSRT profiles (Eqn. 9) in the horizontal plane measured with the BSRT together with the $1 \sigma$ standard deviation over the bunches with the same excitation amplitude $n \cdot \Delta A$ indicated as an envelope for each amplitude. For all values the moving average profiles are used without any further averaging afterwards. 

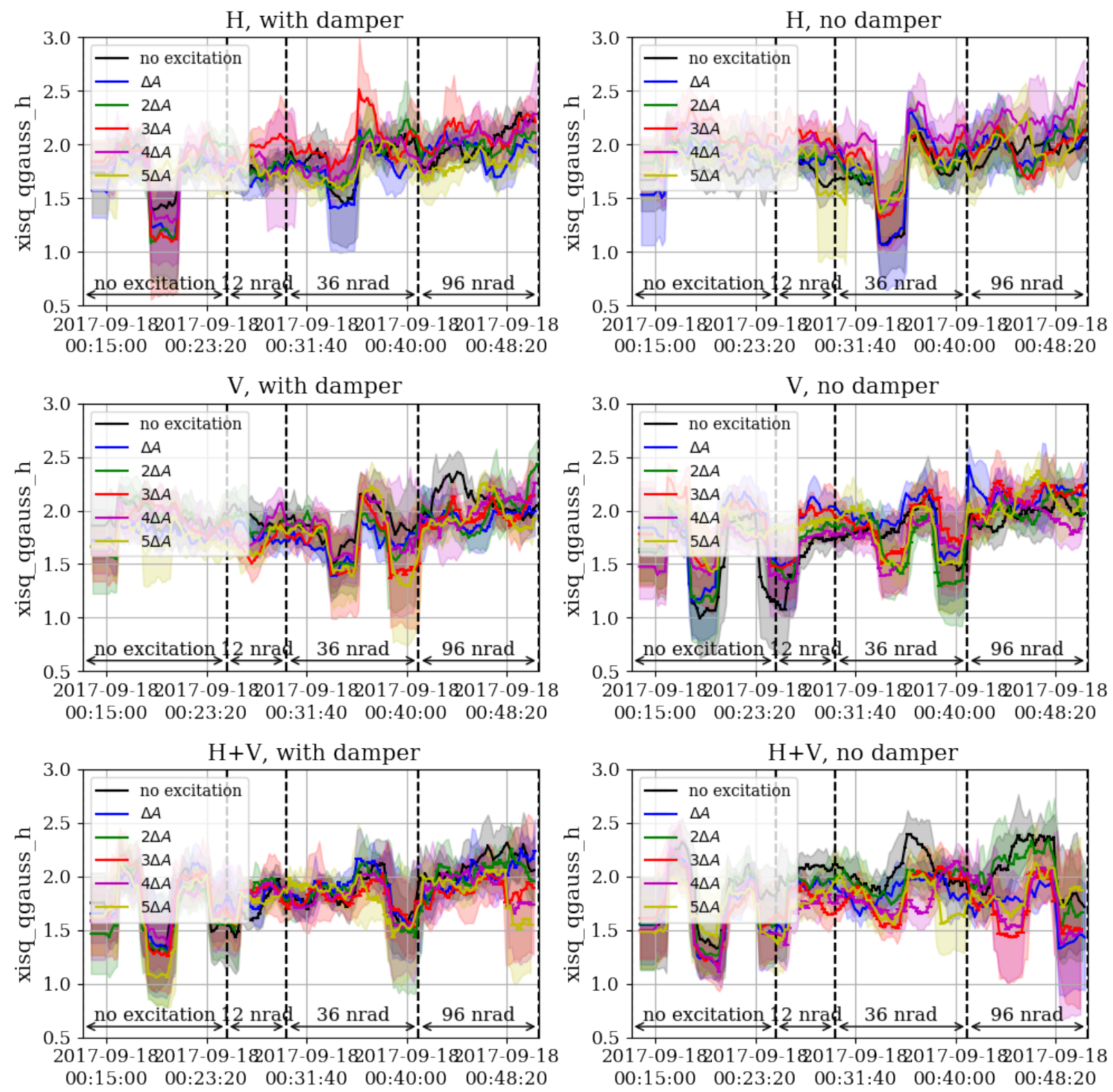

Figure 116. $8^{\text {th }}$ turn pulsing: normalized $\chi^{2}$ of q-Gaussian fit of the BSRT profiles (Eqn. 9) in the horizontal plane measured with the BSRT together with the $1 \sigma$ standard deviation over the bunches with the same excitation amplitude $n \cdot \Delta A$ indicated as an envelope for each amplitude. For all values the moving average profiles are used without any further averaging afterwards. The $\chi^{2}$ stays comparably constant indicating that the quality of the fit also stays unchanged during the entire excitation period. 



Figure 117. $8^{\text {th }}$ turn pulsing: correlation coefficient between fit parameter $a$ and $q$ from q-Gaussian fit of the BSRT profiles (Eqn. 9) in the horizontal plane measured with the BSRT together with the $1 \sigma$ standard deviation over the bunches with the same excitation amplitude $n \cdot \Delta A$ indicated as an envelope for each amplitude. For all values the moving average profiles are used without any further averaging afterwards. 
c. $8^{\text {th }}$ turn pulsing: BSRT profiles - q-Gaussian fit parameters in the vertical plane
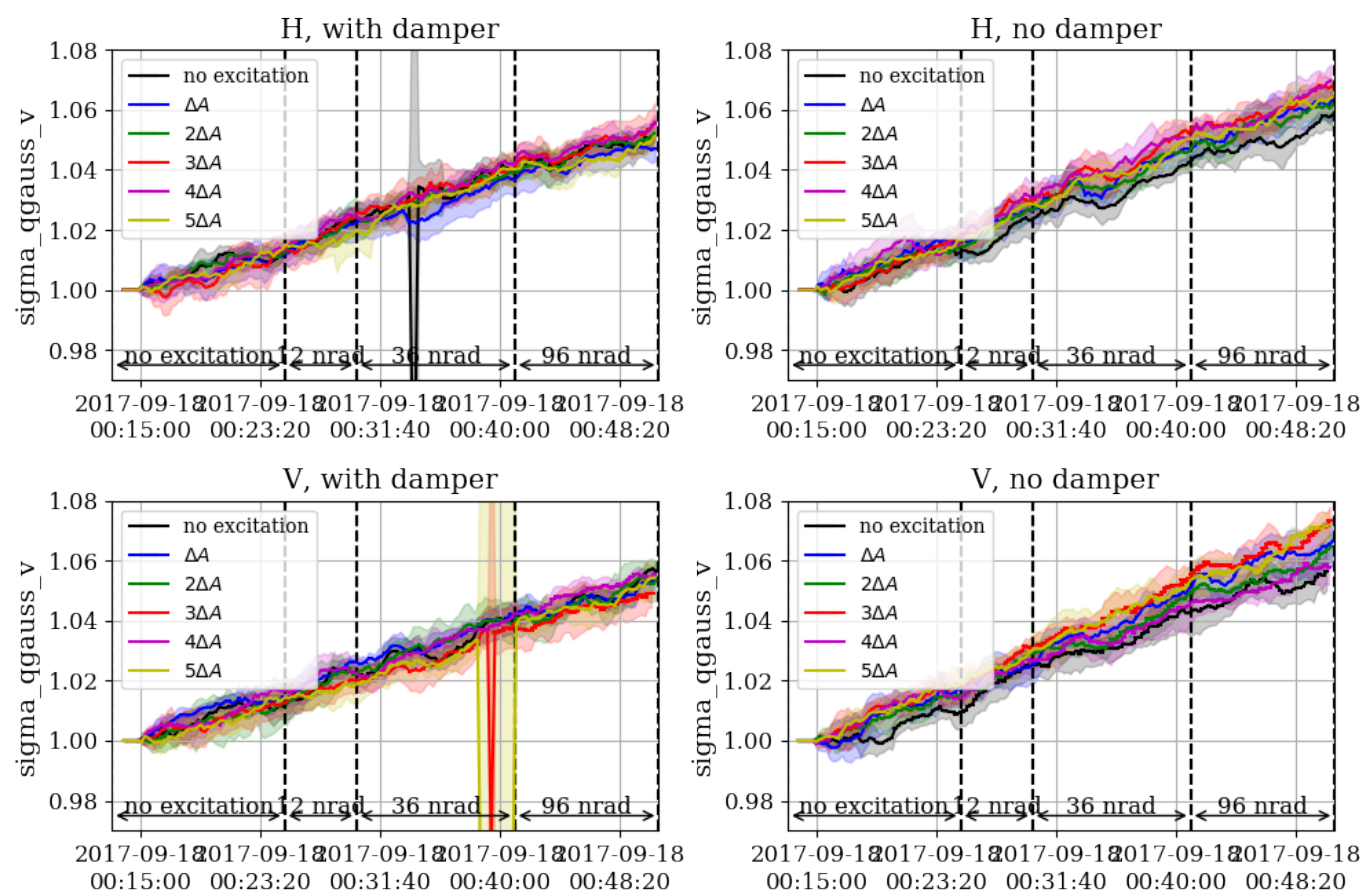

2017-09-1\&017-09-1\&017-09-1\&017-09-1\&017-09-18 00:15:00 00:23:20 00:31:40 00:40:00 00:48:20 00:15:00 00:23:20 00:31:40 00:40:00 00:48:20
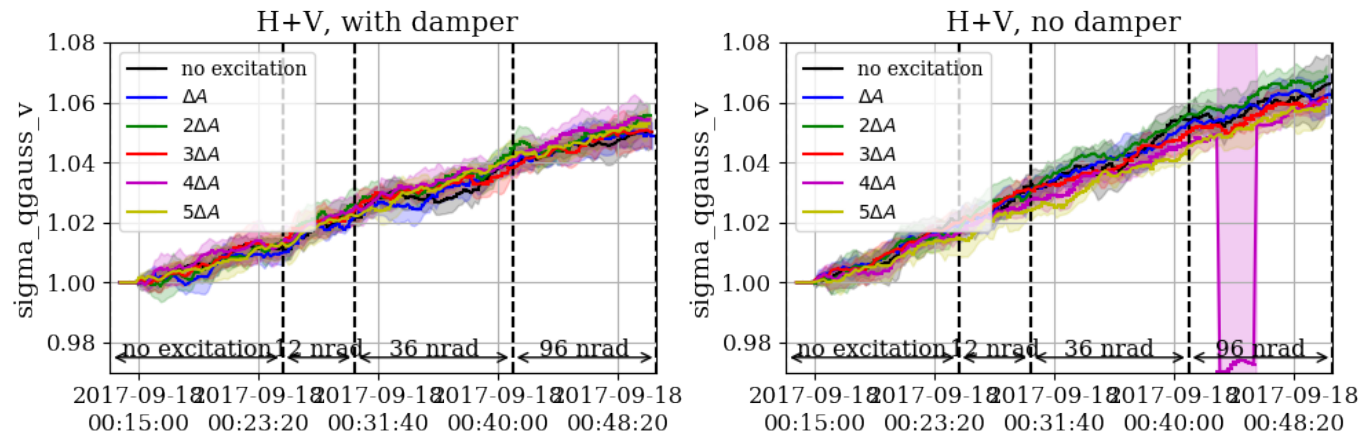

Figure 118. $8^{\text {th }}$ turn pulsing: relative beam sigma from q-Gaussian fit of the BSRT profiles (Eqn. 9) in the vertical plane measured with the BSRT together with the $1 \sigma$ standard deviation over the bunches with the same excitation amplitude $n \cdot \Delta A$ indicated as an envelope for each amplitude. For all values the moving average profiles are used without any further averaging afterwards. 

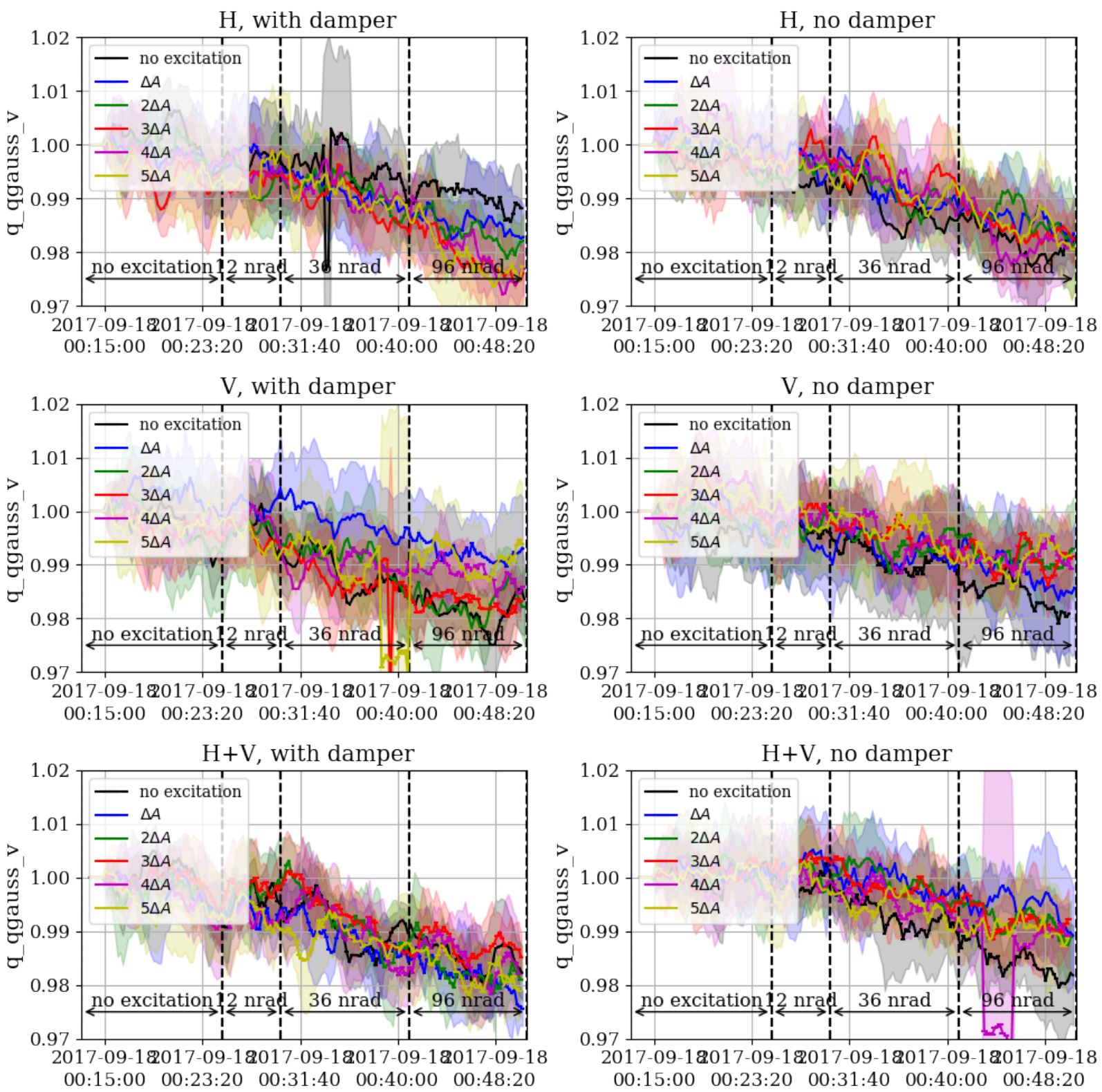

Figure 119. $8^{\text {th }}$ turn pulsing: relative fit parameter $q$ from q-Gaussian fit of the BSRT profiles (Eqn. 9) in the vertical plane measured with the BSRT together with the $1 \sigma$ standard deviation over the bunches with the same excitation amplitude $n \cdot \Delta A$ indicated as an envelope for each amplitude. For all values the moving average profiles are used without any further averaging afterwards. 

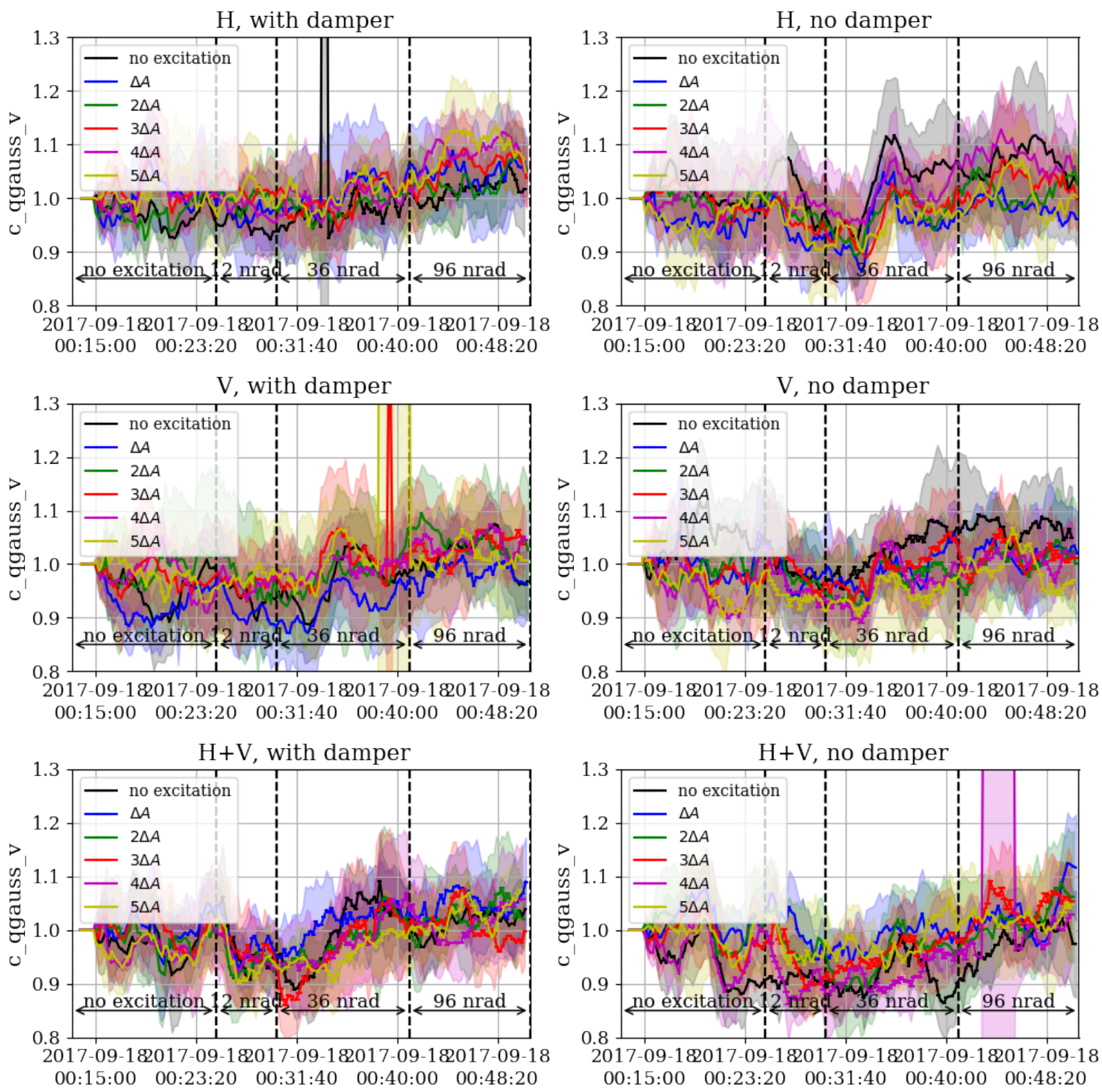

Figure 120. $8^{\text {th }}$ turn pulsing: relative fit parameter $c$ from q-Gaussian fit of the BSRT profiles (Eqn. 9) in the vertical plane measured with the BSRT together with the $1 \sigma$ standard deviation over the bunches with the same excitation amplitude $n \cdot \Delta A$ indicated as an envelope for each amplitude. For all values the moving average profiles are used without any further averaging afterwards. 

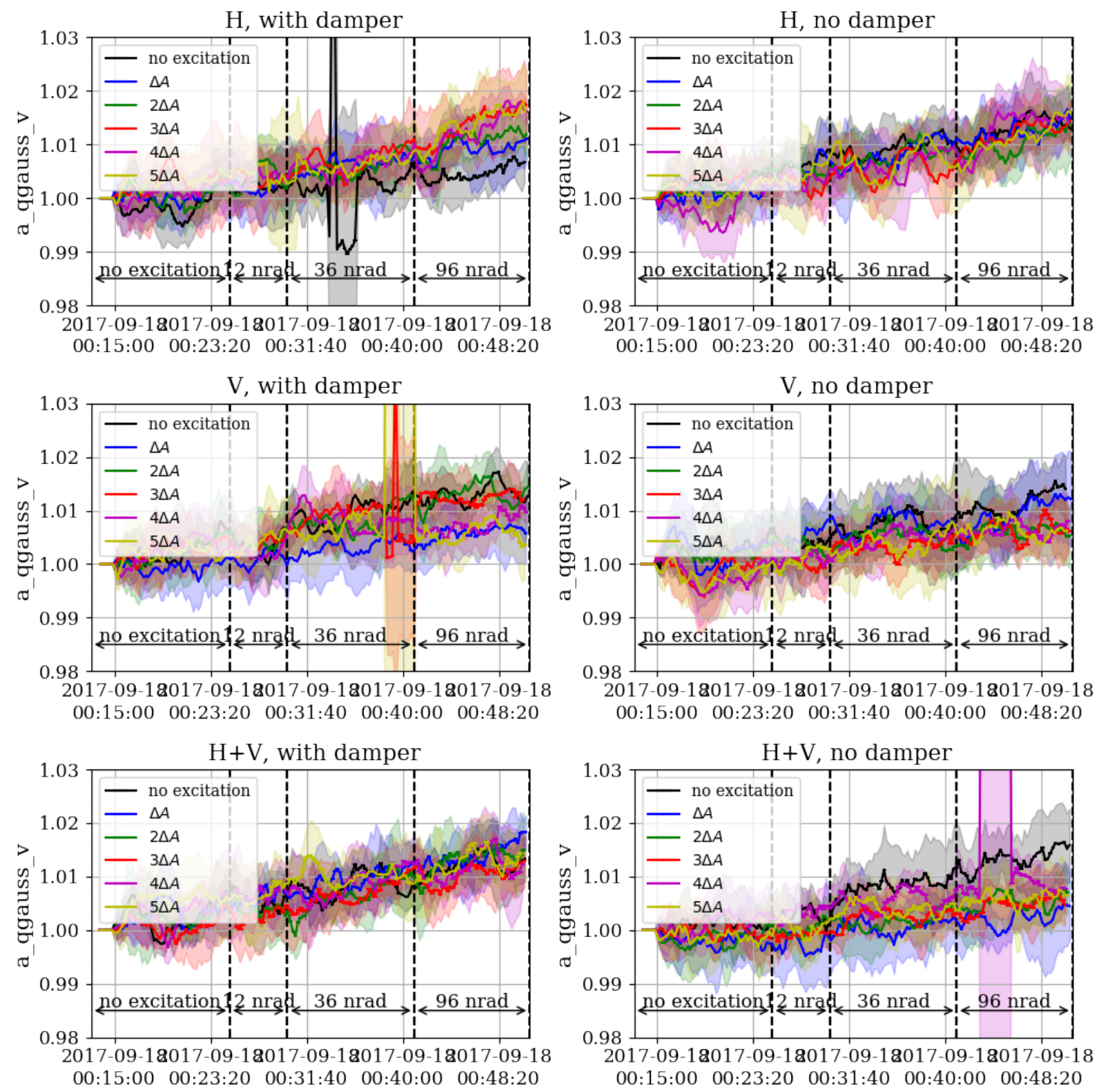

Figure 121. $8^{\text {th }}$ turn pulsing: relative fit parameter $a$ from q-Gaussian fit of the BSRT profiles (Eqn. 9) in the vertical plane measured with the BSRT together with the $1 \sigma$ standard deviation over the bunches with the same excitation amplitude $n \cdot \Delta A$ indicated as an envelope for each amplitude. For all values the moving average profiles are used without any further averaging afterwards. 
$\mathrm{H}$, with damper

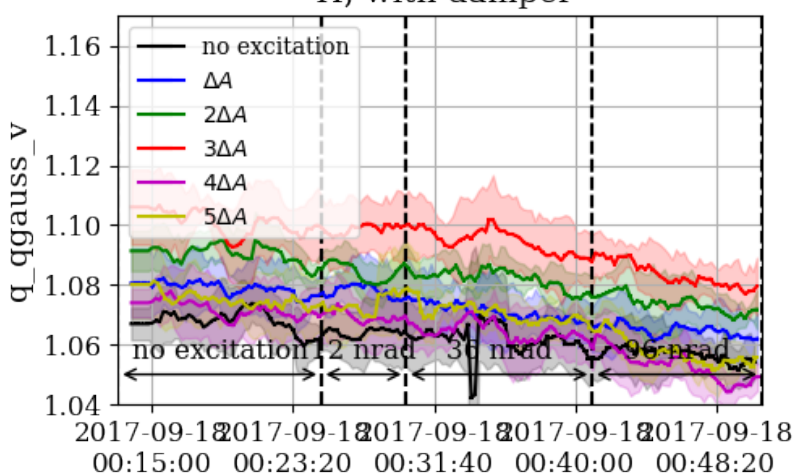

$\mathrm{V}$, with damper



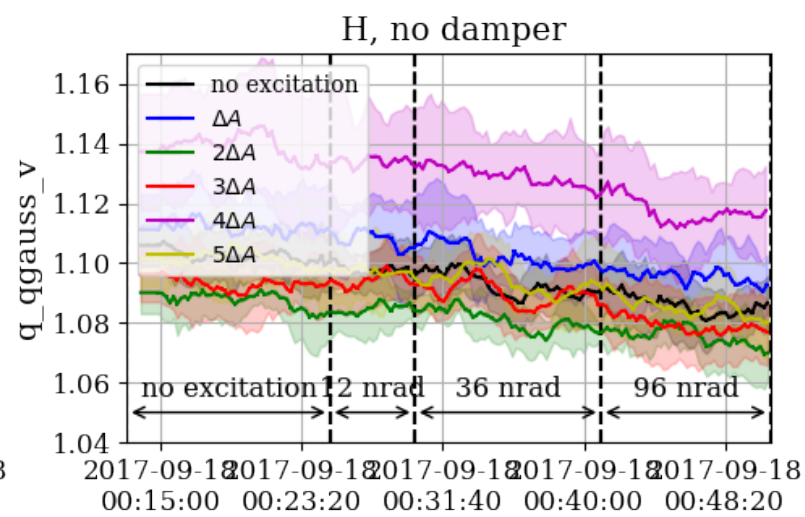
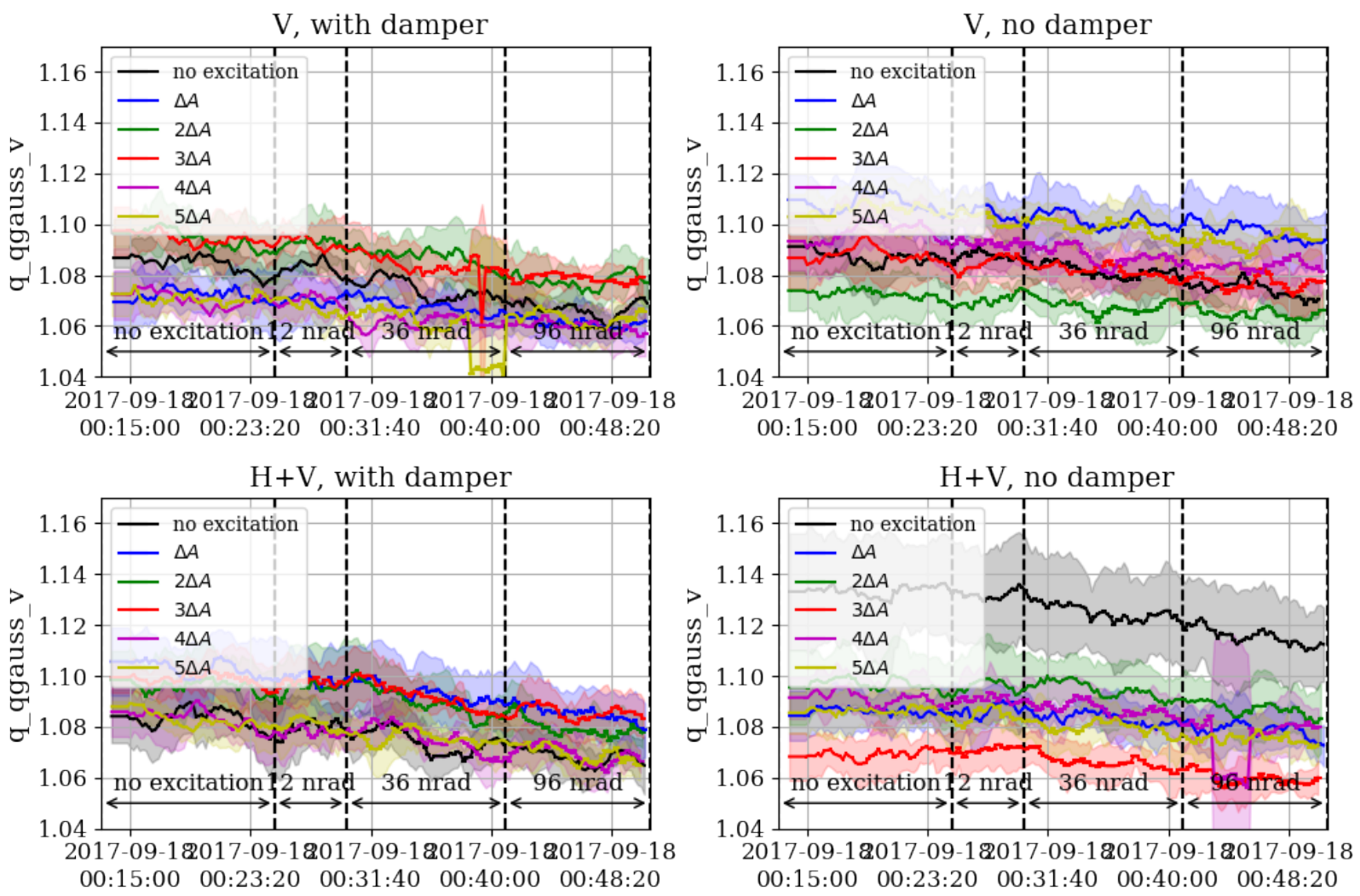

Figure 122. $8^{\text {th }}$ turn pulsing: fit parameter $q$ from q-Gaussian fit of the BSRT profiles (Eqn. 9) in the vertical plane measured with the BSRT together with the $1 \sigma$ standard deviation over the bunches with the same excitation amplitude $n \cdot \Delta A$ indicated as an envelope for each amplitude. For all values the moving average profiles are used without any further averaging afterwards. 



Figure 123. $8^{\text {th }}$ turn pulsing: fit parameter $c$ from q-Gaussian fit of the BSRT profiles (Eqn. 9) in the vertical plane measured with the BSRT together with the $1 \sigma$ standard deviation over the bunches with the same excitation amplitude $n \cdot \Delta A$ indicated as an envelope for each amplitude. For all values the moving average profiles are used without any further averaging afterwards. 



Figure 124. $8^{\text {th }}$ turn pulsing: fit parameter $a$ from q-Gaussian fit of the BSRT profiles (Eqn. 9) in the vertical plane measured with the BSRT together with the $1 \sigma$ standard deviation over the bunches with the same excitation amplitude $n \cdot \Delta A$ indicated as an envelope for each amplitude. For all values the moving average profiles are used without any further averaging afterwards. 

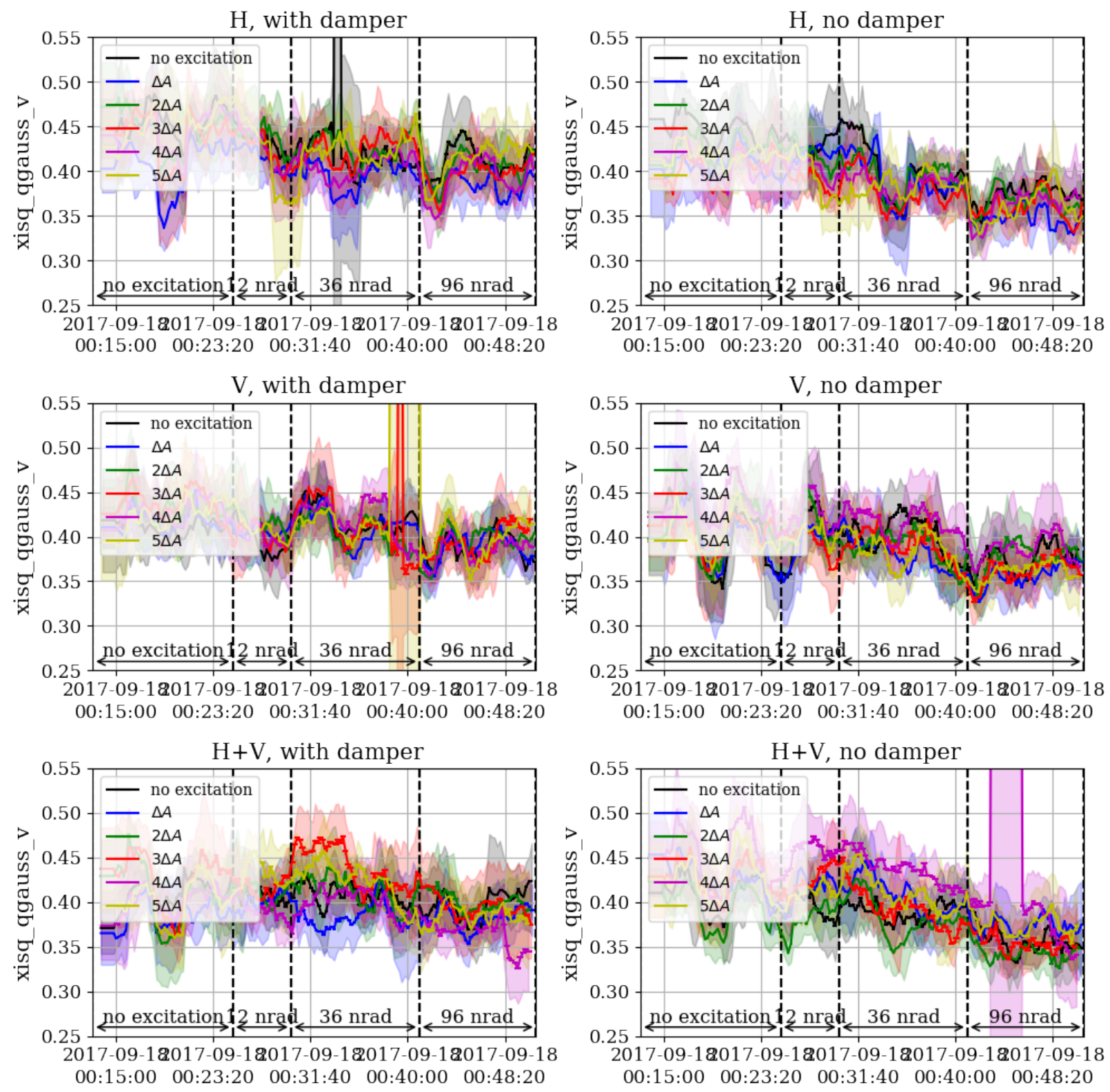

Figure 125. $8^{\text {th }}$ turn pulsing: normalized $\chi^{2}$ of q-Gaussian fit of the BSRT profiles (Eqn. 9) in the vertical plane measured with the BSRT together with the $1 \sigma$ standard deviation over the bunches with the same excitation amplitude $n \cdot \Delta A$ indicated as an envelope for each amplitude. For all values the moving average profiles are used without any further averaging afterwards. The $\chi^{2}$ stays comparably constant indicating that the quality of the fit also stays unchanged during the entire excitation period. 

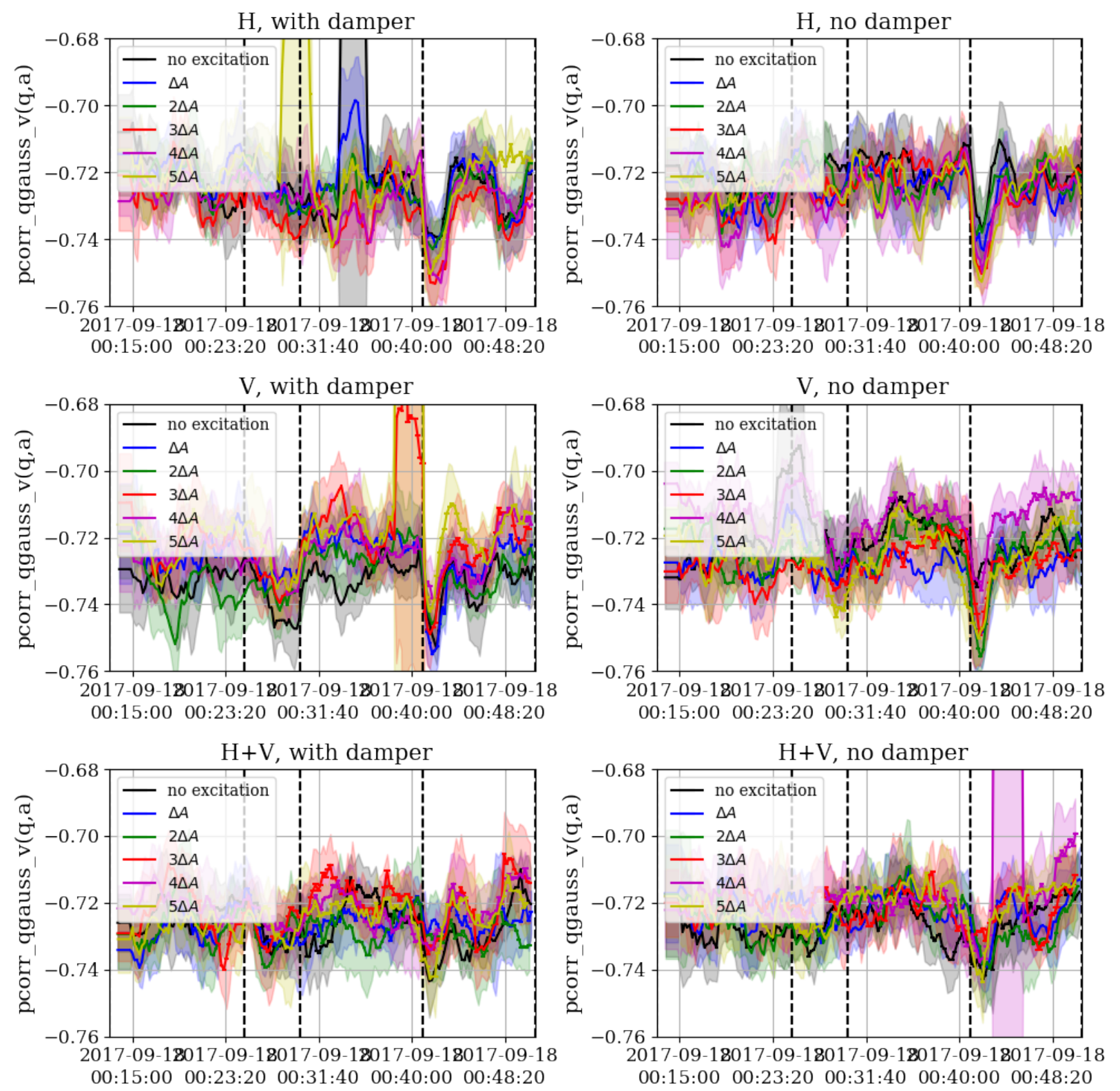

Figure 126. $8^{\text {th }}$ turn pulsing: correlation coefficient between fit parameter $a$ and $q$ from q-Gaussian fit of the BSRT profiles (Eqn. 9) in the vertical plane measured with the BSRT together with the $1 \sigma$ standard deviation over the bunches with the same excitation amplitude $n \cdot \Delta A$ indicated as an envelope for each amplitude. For all values the moving average profiles are used without any further averaging afterwards. 
d. $8^{\text {th }}$ turn pulsing: BSRT profiles of representative bunches

no damper, no excitation

H plane, slot 804 - 2017-09-18 00:40:57, ref slot 804 - 2017-09-18 00:21:04
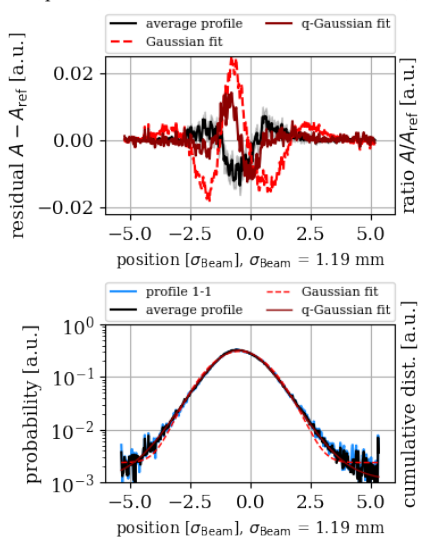

no damper, no excitation

V plane, slot 804 - 2017-09-18 00:40:57, ref slot 804 - 2017-09-18 00:21:04

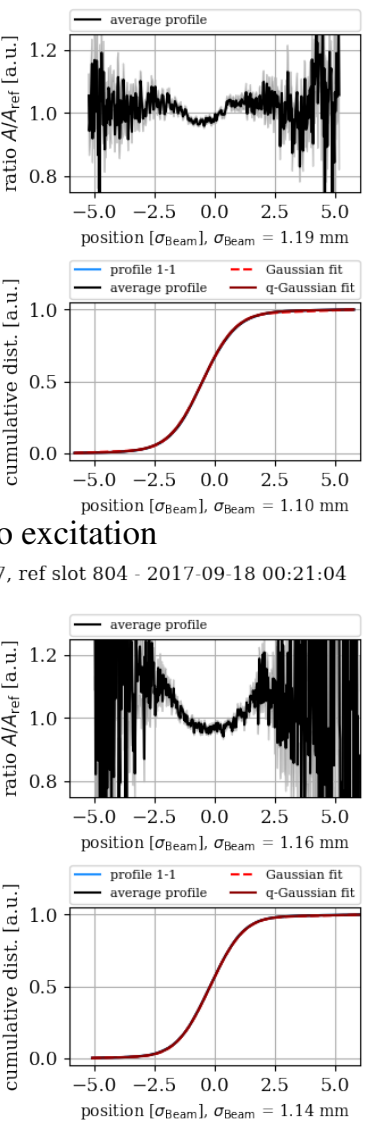

no damper, with excitation $(5 \cdot \Delta A)$ in $\mathrm{H}$

H plane, slot 638 - 2017-09-18 00:40:55, ref slot 638 - 2017-09-18 00:21:02

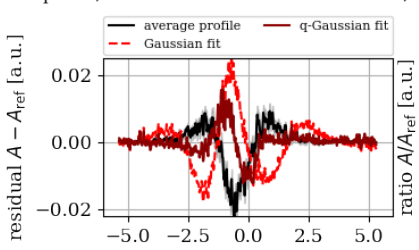

position [ $\left.\sigma_{\text {Beam }}\right], \sigma_{\text {Beam }}=1.16 \mathrm{~mm}$

- profile 1-1
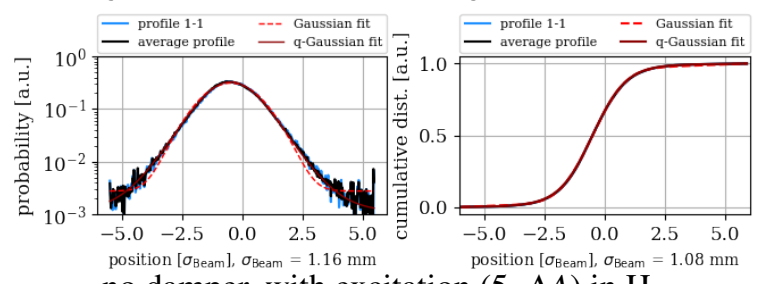

no damper, with excitation $(5 \cdot \Delta A)$ in $\mathrm{H}$

V plane, slot 638 - 2017-09-18 00:40:55, ref slot 638 - 2017-09-18 00:21:02


Figure 127. $8^{\text {th }}$ turn pulsing: BSRT profile in the horizontal (top) and vertical (bottom) plane of one bunch without resonant excitation (left) and one bunch experiencing the maximum excitation (right) for an excitation only in H. For all bunches the damper is not active. The residual is taken in respect to the start (2017-09-18 00:21) and end (201709-18 00:41) of the $8^{\text {th }}$ turns pulsing with a few minutes subtracted in order to account for the moving average of the profiles over 11 time stamps. The $\mathrm{x}$-axis is the position of the BSRT image projection and not the proton beam sigma. 
no damper, no excitation

H plane, slot 1698 - 2017-09-18 00:40:47, ref slot 1698 - 2017-09-18 00:21:08

no damper, no excitation

V plane, slot 1698 - 2017-09-18 00:40:47, ref slot 1698 - 2017-09-18 00:21:08

- profle 1-1 --- Gaussian fit

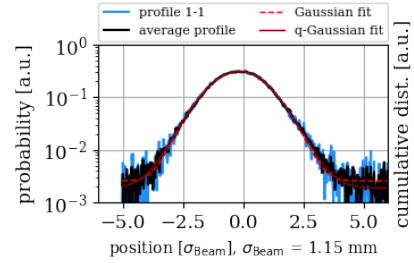



- profile 1-1 -- Gaussian fit


ition $\left[\sigma_{\text {Beam }}\right] \sigma_{\text {Beam }}=1.15 \mathrm{~mm}$

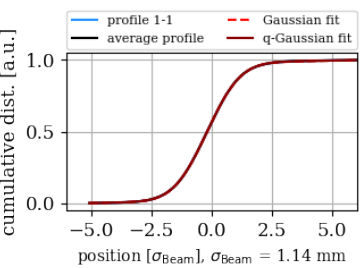

no damper, with excitation $(5 \cdot \Delta A)$ in $\mathrm{V}$

H plane, slot 1532 - 2017-09-18 00:40:59, ref slot 1532 - 2017-09-18 00:21:06
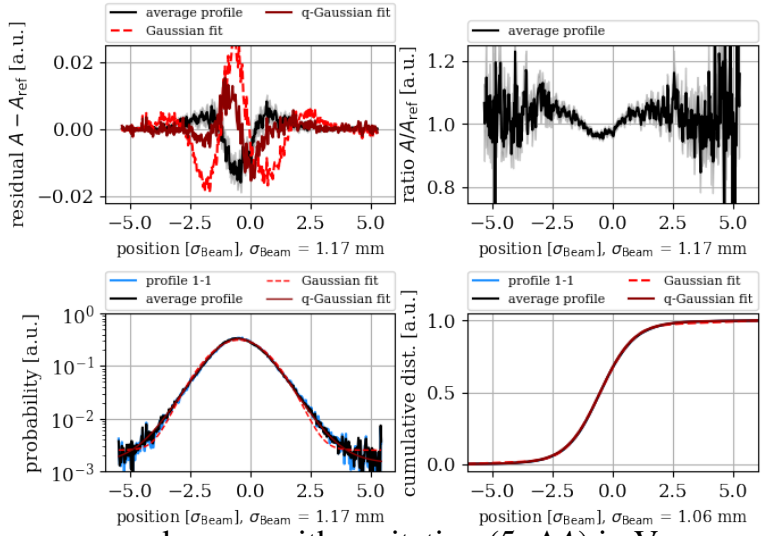

no damper, with excitation $(5 \cdot \Delta A)$ in $\mathrm{V}$

V plane, slot 1532 - 2017-09-18 00:40:59, ref slot 1532 - 2017-09-18 00:21:06

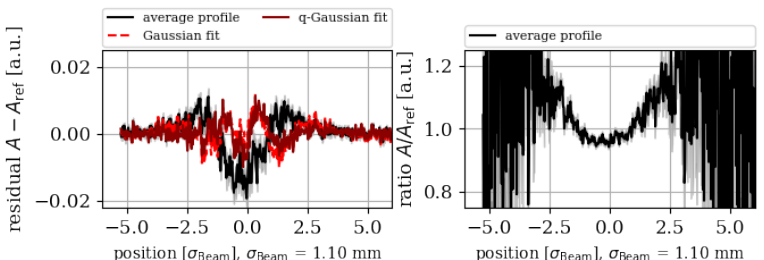

$\left[\sigma_{\text {Beam }}\right], \sigma_{\text {Beam }}=1.10 \mathrm{~mm}$ 二 profile 1-1

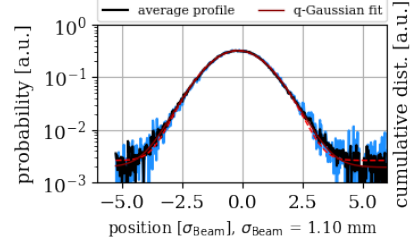

position $\left[\sigma_{\text {Beam }}\right], \sigma_{\text {Beam }}=1.10 \mathrm{~mm}$



Figure 128. $8^{\text {th }}$ turn pulsing: BSRT profile in the horizontal (top) and vertical (bottom) plane of one bunch without resonant excitation (left) and one bunch experiencing the maximum excitation (right) for an excitation only in V. For all bunches the damper is not active. The residual is taken in respect to the start (2017-09-18 00:21) and end (201709-18 00:41) of the $8^{\text {th }}$ turn pulsing with a few minutes subtracted in order to account for the moving average of the profiles over 11 time stamps. Note that 22:56 is also after the closed orbit shift in the vertical plane. The $\mathrm{x}$-axis is the position of the BSRT image projection and not the proton beam sigma. 
no damper, no excitation

H plane, slot 2862 - 2017-09-18 00:40:52, ref slot 2862 - 2017-09-18 00:21:12
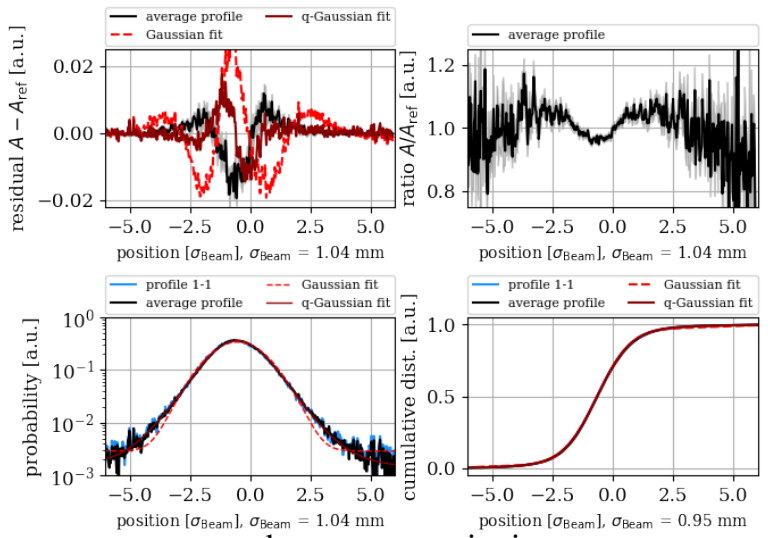

no damper, no excitation

V plane, slot 2862 - 2017-09-18 00:40:52, ref slot 2862 - 2017-09-18 00:21:12



no damper, with excitation $(5 \cdot \Delta A)$ in $\mathrm{H}+\mathrm{V}$

H plane, slot 2696 - 2017-09-18 00:40:50, ref slot 2696 - 2017-09-18 00:21:10
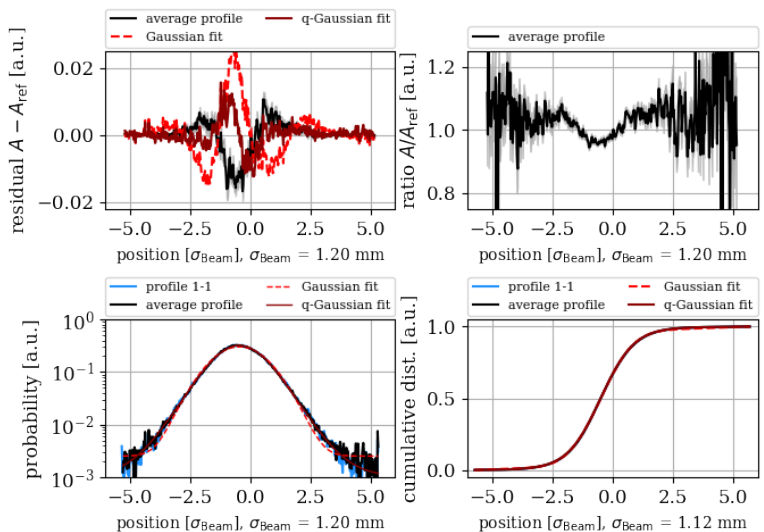
position $\left[\sigma_{\text {Beam }}\right], \sigma_{\text {Beam }}=1.20 \mathrm{~mm}$ position $\left[\sigma_{\text {Beam }}\right], \sigma_{\text {Beam }}=1.12$
no damper, with excitation $(5 \cdot \Delta A)$ in $\mathrm{H}+\mathrm{V}$

V plane, slot 2696 - 2017-09-18 00:40:50, ref slot 2696 - 2017-09-18 00:21:10
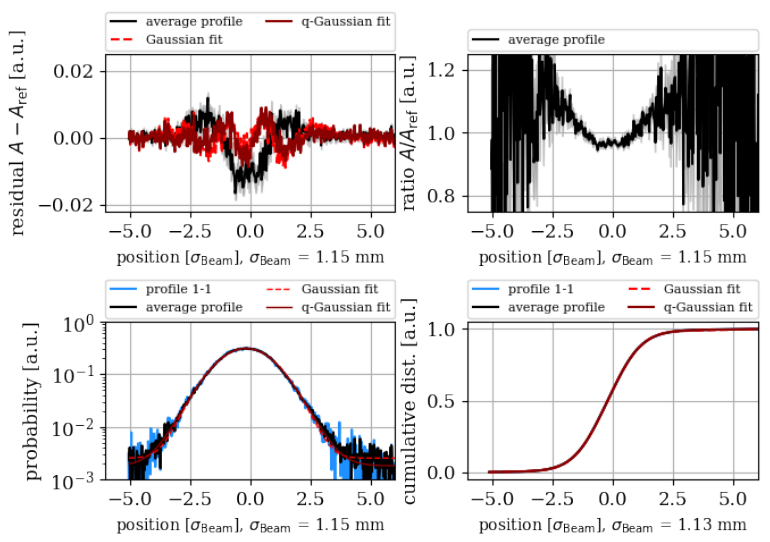

Figure 129. $8^{\text {th }}$ turn pulsing: BSRT profile in the horizontal (top) and vertical (bottom) plane of one bunch without resonant excitation (left) and one bunch experiencing the maximum excitation (right) for an excitation only in $\mathrm{H}+\mathrm{V}$. For all bunches the damper is not active. The residual is taken in respect to the start (2017-09-18 00:21) and end (2017-09-18 00:41) of the $8^{\text {th }}$ turn pulsing with a few minutes subtracted in order to account for the moving average of the profiles over 11 time stamps. Note that 22:56 is also after the closed orbit shift in the vertical plane. The $\mathrm{x}$-axis is the position of the BSRT image projection and not the proton beam sigma. 
with damper, no excitation

H plane, slot 442 - 2017-09-17 23:22:52, ref slot 442 - 2017-09-17 22:57:11


with damper, no excitation

V plane, slot 442 - 2017-09-18 00:40:53, ref slot 442 - 2017-09-18 00:21:00
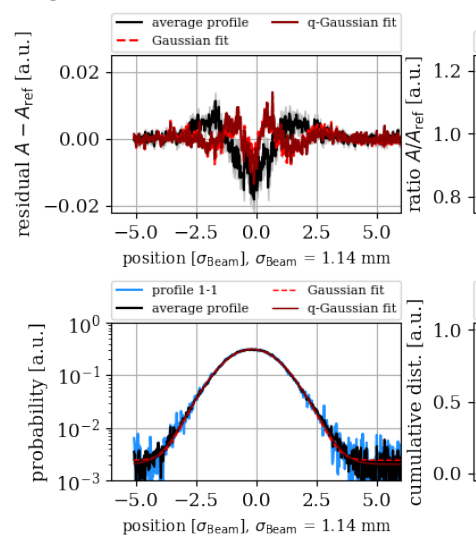
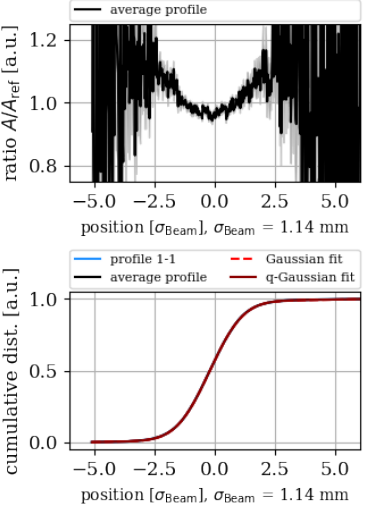

with damper, with excitation $(5 \cdot \Delta A)$ in $\mathrm{H}$

H plane, slot 608 - 2017-09-18 00:40:55, ref slot 608 - 2017-09-18 00:21:02
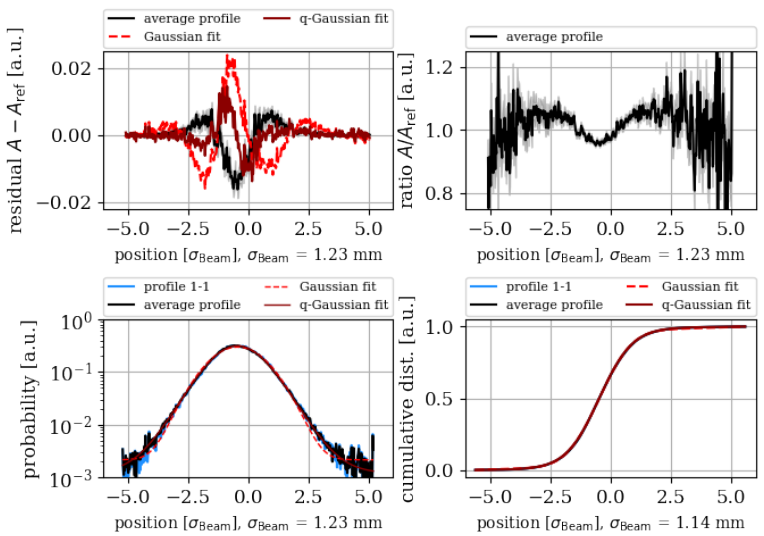
position $\left[\sigma_{\text {Beam }}\right], \sigma_{\text {Beam }}=1.23 \mathrm{~mm}$ with damper, with excitation $(5 \cdot \Delta A)$ in $\mathrm{H}$
with

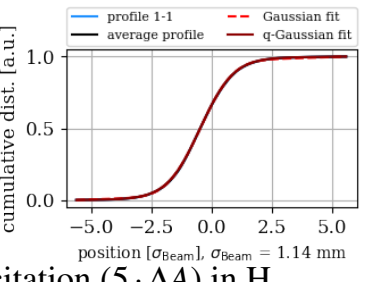

V plane, slot 608 - 2017-09-18 00:40:55, ref slot 608 - 2017-09-18 00:21:02
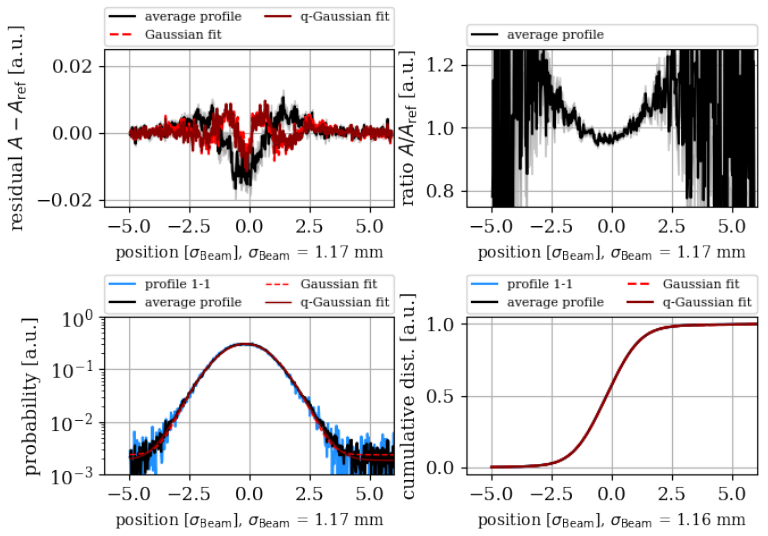

Figure 130. $8^{\text {th }}$ turn pulsing: BSRT profile in the horizontal (top) and vertical (bottom) plane of one bunch without resonant excitation (left) and one bunch experiencing the maximum excitation (right) for an excitation only in H. For all bunches the damper is active. The residual is taken in respect to the start (2017-09-18 00:21) and end (2017-09-18 00:41) of the $8^{\text {th }}$ turn pulsing with a few minutes subtracted in order to account for the moving average of the profiles over 11 time stamps. The $\mathrm{x}$-axis is the position of the BSRT image projection and not the proton beam sigma. 
with damper, no excitation

H plane, slot 1336 - 2017-09-18 00:40:57, ref slot 1336 - 2017-09-18 00:21:04
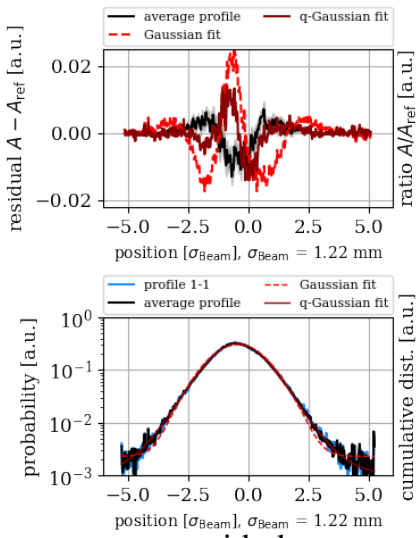

with damper, no excitation

V plane, slot 1336 - 2017-09-18 00:40:57, ref slot 1336 - 2017-09-18 00:21:04

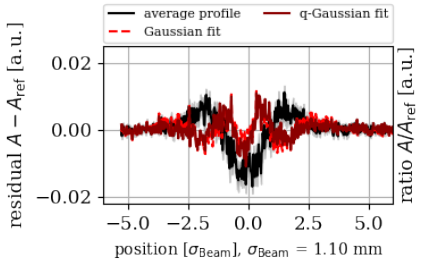

— proflie 1-1 --- Gaussian fit

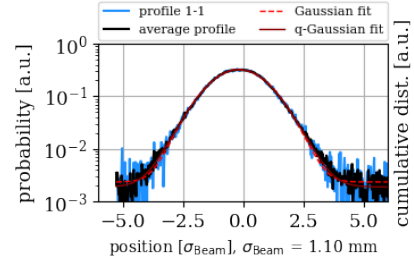

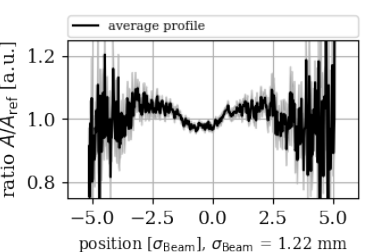


with damper, with excitation $(5 \cdot \Delta A)$ in $\mathrm{V}$

H plane, slot 1502 - 2017-09-18 00:40:59, ref slot 1502 - 2017-09-18 00:21:06
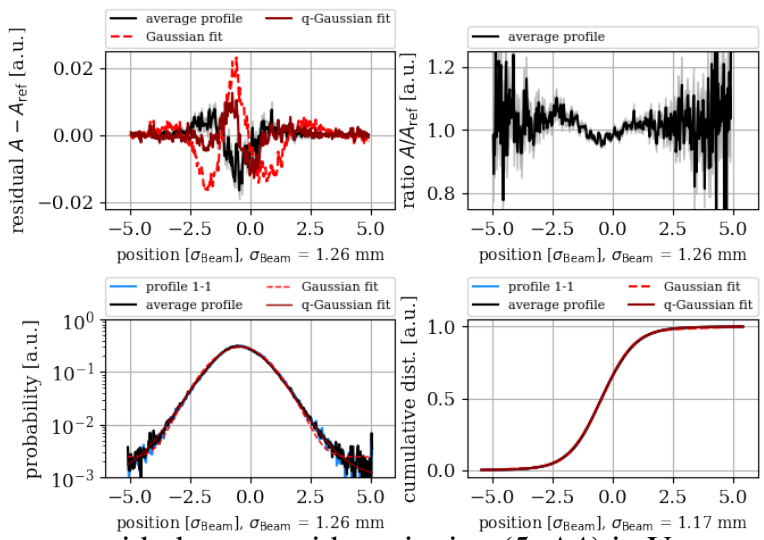

position $\left[\sigma_{\text {Beam }}, \sigma_{\text {Beam }}=1.26 \mathrm{~mm}\right.$ position $\left[\sigma_{\text {Beam }}\right], \sigma_{\text {Beam }}=1.17 \mathrm{~mm}$ with damper, with excitation $(5 \cdot \Delta A)$ in $\mathrm{V}$

V plane, slot 1502 - 2017-09-18 00:40:59, ref slot 1502 - 2017-09-18 00:21:06
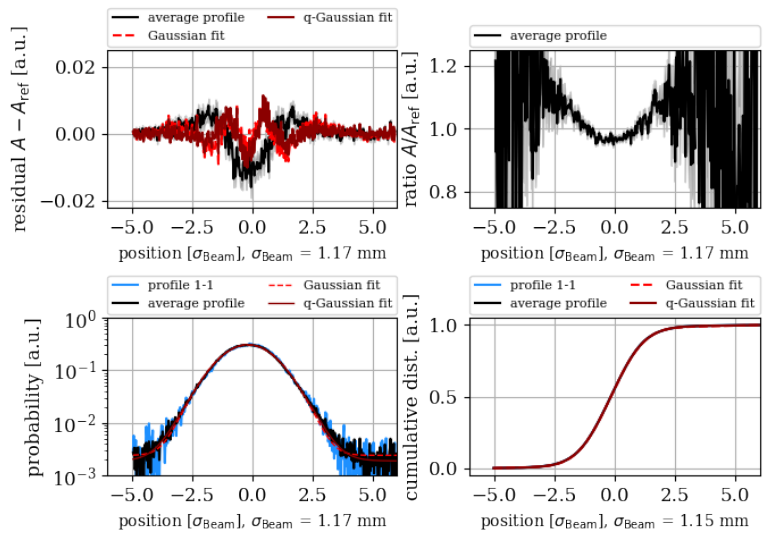

Figure 131. $8^{\text {th }}$ turn pulsing: BSRT profile in the horizontal (top) and vertical (bottom) plane of one bunch without resonant excitation (left) and one bunch experiencing the maximum excitation (right) for an excitation only in V. For all bunches the damper is active. The residual is taken in respect to the start (2017-09-18 00:21) and end (2017-09-18 00:41) of the $8^{\text {th }}$ turn pulsing with a few minutes subtracted in order to account for the moving average of the profiles over 11 time stamps. Note that $22: 56$ is also after the closed orbit shift in the vertical plane. The $\mathrm{x}$-axis is the position of the BSRT image projection and not the proton beam sigma. 
with damper, no excitation

H plane, slot 2500 - 2017-09-18 00:40:48, ref slot 2500 - 2017-09-18 00:21:08


with damper, no excitation

V plane, slot 2500 - 2017-09-18 00:40:48, ref slot 2500 - 2017-09-18 00:21:08

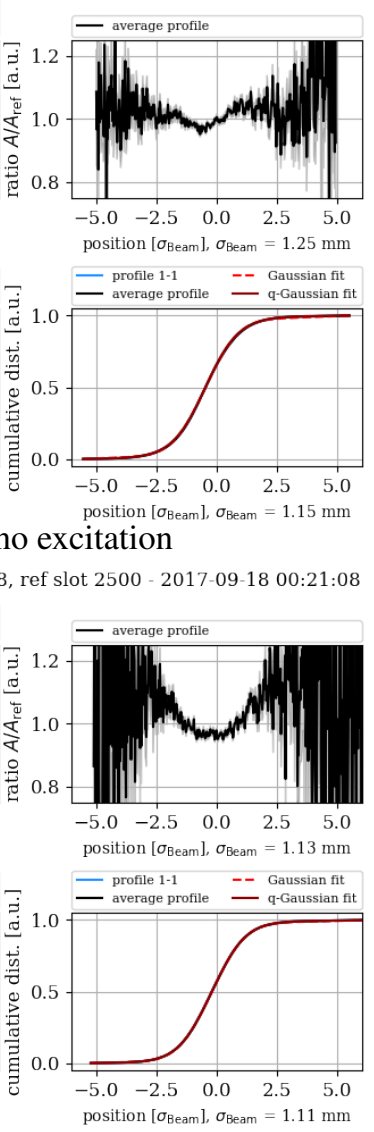

with damper, with excitation $(5 \cdot \Delta A)$ in $\mathrm{H}+\mathrm{V}$

H plane, slot 2666 - 2017-09-18 00:40:50, ref slot 2666 - 2017-09-18 00:21:10


with damper, with excitation $(5 \cdot \Delta A)$ in $\mathrm{H}+\mathrm{V}$

V plane, slot 2666 - 2017-09-18 00:40:50, ref slot 2666 - 2017-09-18 00:21:10
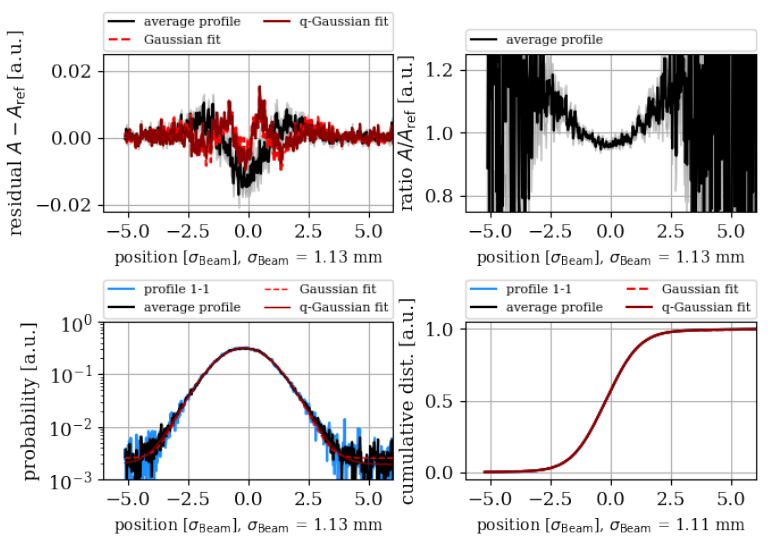

Figure 132. $8^{\text {th }}$ turn pulsing: BSRT profile in the horizontal (top) and vertical (bottom) plane of one bunch without resonant excitation (left) and one bunch experiencing the maximum excitation (right) for an excitation only in $\mathrm{H}+\mathrm{V}$. For all bunches the damper is active. The residual is taken in respect to the start (2017-09-18 00:21) and end (201709-18 00:41) of the $8^{\text {th }}$ turn pulsing with a few minutes subtracted in order to account for the moving average of the profiles over 11 time stamps. The x-axis is the position of the BSRT image projection and not the proton beam sigma. 


\section{4. $8^{\text {th }}$ turn pulsing: FMA Analysis for 2017 optics and tune}


Figure 133. FMA analysis in frequency space for $8^{\text {th }}$ turn pulsing based on the 2017 injection optics with no machine errors and a tune of $(62.27,60.295)$. The excitation is $96 \mathrm{nrad}$ in each/both planes. For a horizontal excitation the $8 Q_{x}-4 Q_{y}$ resonance is excited, for a vertical excitation the $16 Q_{y}$ and for an excitation in both planes obviously both resonances. 